\title{
QUADRICEPS FUNCTION IN ACL RECONSTRUCTED PATIENTS WITH AND WITHOUT KNEE OSTEOARTHRITIS
}

\author{
A Dissertation \\ Presented to \\ The Faculty of the Curry School of Education \\ University of Virginia \\ In Partial Fulfillment \\ Of the Requirements for the Degree \\ Doctor of Philosophy
}

by

Grant Edward Norte, $\mathrm{PhD}$

May 2016 
(C) Copyright by

Grant Edward Norte

All Rights Reserved

May 2016 


\begin{abstract}
Post-traumatic quadriceps dysfunction is well-documented following anterior cruciate ligament reconstruction (ACL-R), and is associated with impairments detrimental to joint-specific and global health, including decreased physical activity, accelerated onset of knee joint osteoarthritis, and decreased quality of life. Since articular cartilage degeneration is irreversible, the hallmark for prevention is early detection with thorough evaluation of quadriceps neuromuscular function. Neuromuscular adaptations are theorized to arise from alterations in spinal-mediated and corticospinal pathways, and if unaddressed, may present a limiting factor in recovery from ACL$\mathrm{R}$. The specific origins of impairment have been theorized as a way to address subtle underlying factors impeding the recovery of quadriceps function following ACL-R. By understanding the temporal nature of neuromuscular adaptations, clinicians and researchers can improve patient care. The focus of manuscript 1 was to compare quadriceps neuromuscular function at clinically relevant time points following ACL-R, including patients who experienced post-traumatic knee osteoarthritis. We found that patients early ( $<1$ year), late $(>2$ years), and with osteoarthritis after ACL-R exhibited quadriceps weakness and decreased corticospinal input to the quadriceps compared to healthy individuals. The focus of manuscript 2 was to identify the relationship between objective measures of quadriceps function and patient-reported outcomes at clinically relevant time points following ACL-R, including patients who experienced post-traumatic knee osteoarthritis. We found that perceived knee function and global health status were best explained by objective measures of quadriceps function in patients early and with osteoarthritis after ACLR. Both limb symmetry and unilateral limb performance were meaningful to patients early, and unilateral limb performance was meaningful to patients with osteoarthritis after ACL-R.
\end{abstract}


Measures of isokinetic quadriceps strength (torque, work, power) consistently demonstrated the strongest relationships with patient-reported outcomes. The focus of manuscript 3 was to investigate the underlying constructs of lower extremity muscle function that uniquely describe aspects of quadriceps neuromuscular function in patients after ACL-R. We found that unique constructs of peripheral, central, and combined peripheral and central muscle function are likely to exist in ACL-R patients. Quadriceps function (total work at $90 \% \mathrm{sec}$, active motor threshold, and central activation) of the involved limb was able to discriminate best between ACL-R patients and healthy individuals compared to the uninvolved limb or limb symmetry. It is unclear if early changes in strength, endurance, voluntary activation, and corticospinal excitability perpetuate long-term muscle dysfunction; however, the temporal relationships of these measures may be a contributing factor to long-term outcomes. If left unaddressed, the progressive nature of contributing factors may result in irreversible joint injury. 
Kinesiology Department

Curry School of Education

University of Virginia

Charlottesville, Virginia

\section{APPROVAL OF THE DISSERTATION}

This dissertation, "Quadriceps Function in ACL Reconstructed Patients With and Without Knee Osteoarthritis," has been approved by the Graduate Faculty of the Curry School of Education in partial fulfillment of the requirements for the degree of Doctor of Philosophy

Joe Hart, Committee Chair

Jay Hertel, Committee Member

Sue Saliba, Committee Member

David Diduch, Committee Member 


\section{ACKNOWLEDGMENTS}

I would like to first thank my dissertation committee for their continuous support over the last four years. The invaluable education, mentorship, and friendship I have received from each of you will undoubtedly help shape me as I begin my (hopefully successful) career. I would specifically like to thank Joe Hart for the guidance, encouragement, and humor you have provided me over the eight years we have known each other. You pushed me to be better and to “think big" everyday, and I am grateful for that. I would like to thank one of my earliest mentors and friend, Kavin Tsang. You were the first to put me on a path to success, and without your guidance, I would not be in the position I am today - thanks for teaching me to "man up." I would like to thank the Eastern Athletic Trainers' Association, Mid-Atlantic Athletic Trainers' Association, and Curry School of Education for providing funds to complete this study.

With any success, comes a strong support system. Thank you to my friends and colleagues, Neal Glaviano, John Goetschius, and Mark Feger. We've been there to support each other through the best and the worst of the past four years, and I couldn't have asked for a better class to go through this experience with. To Chris Kuenze, thank you for the continual mentorship you have provided me from start to finish. Your friendship and guidance have had a significant influence on my growth in academia. Finally, I would like to thank my family for their unconditional support. My parents, Darryll and Patti, have never questioned my decisions, but always supported them. To my wife, Shari, thank you for seeing the bigger picture, and putting up with me for the past four years through the ups and downs of $\mathrm{PhD}$ life - I would not be here without you. 


\section{TABLE OF CONTENTS}

\section{SECTION I: FRONT MATTER}

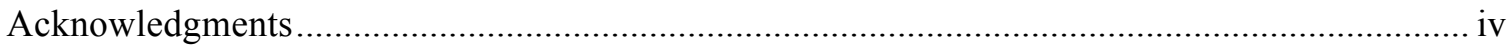

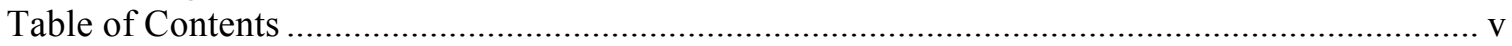

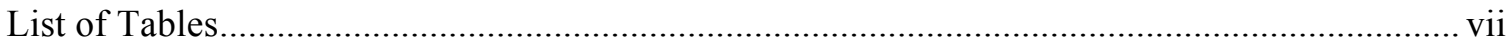

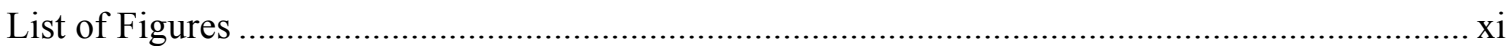

\section{SECTION II: MANUSCRIPTS}

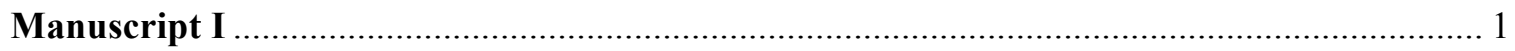

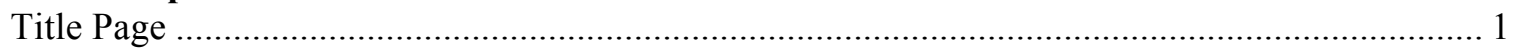

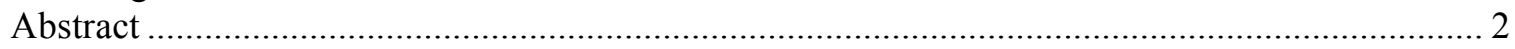

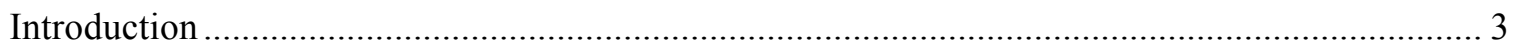

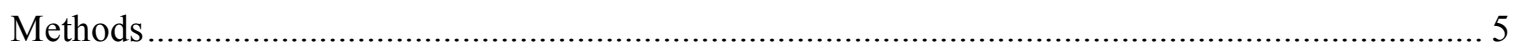

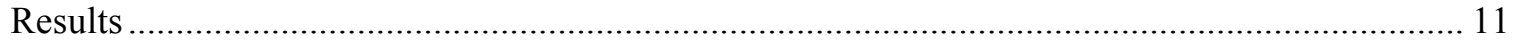

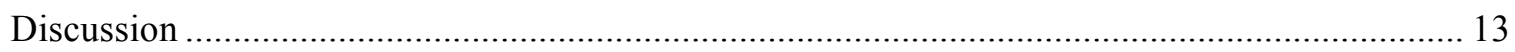

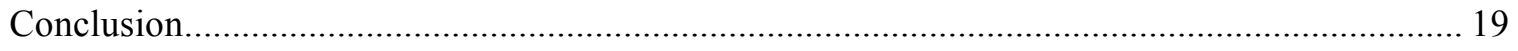

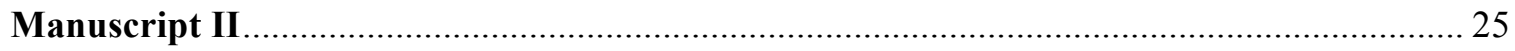

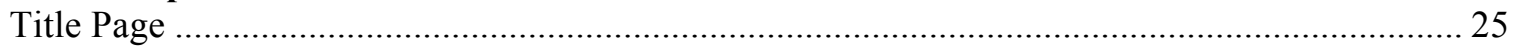

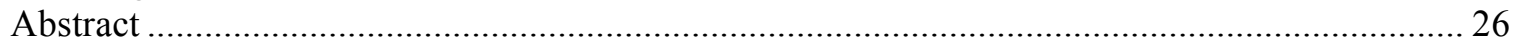

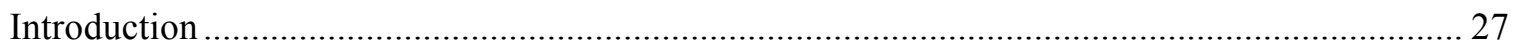

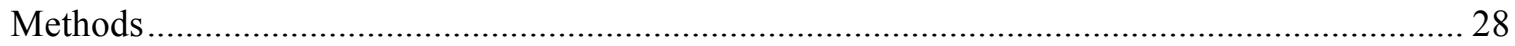

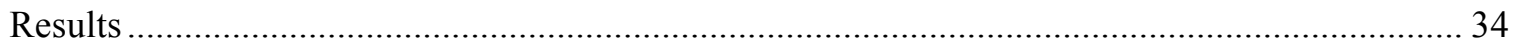

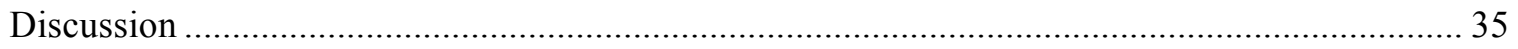

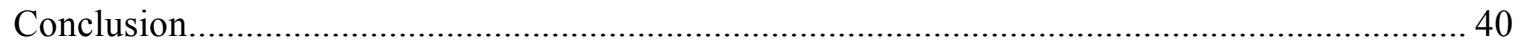

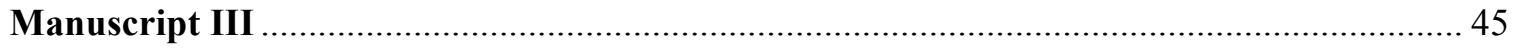

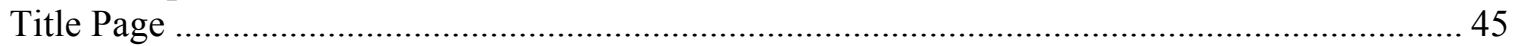

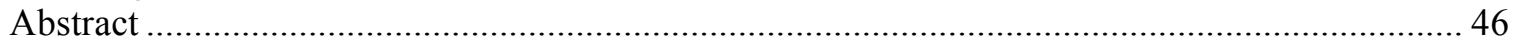

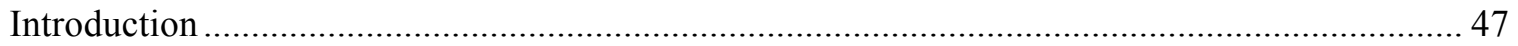

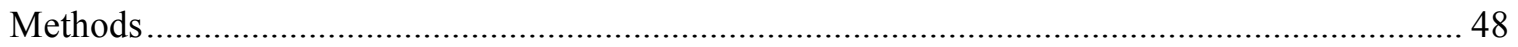

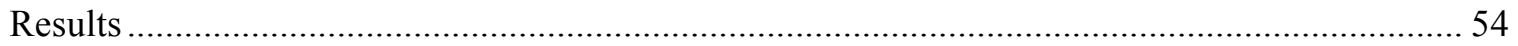

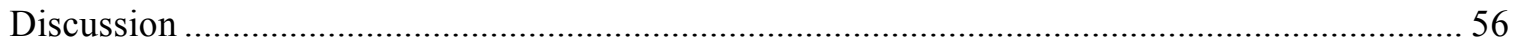

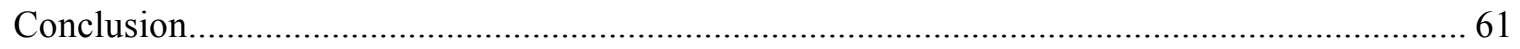

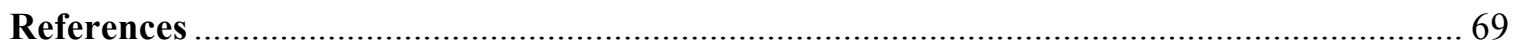




\section{SECTION III: APPENDICES}

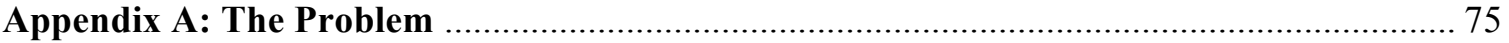

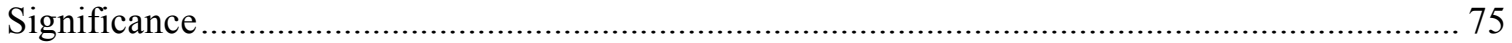

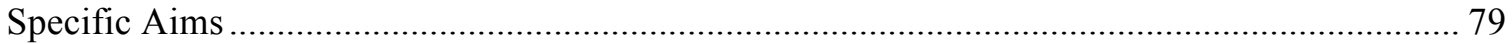

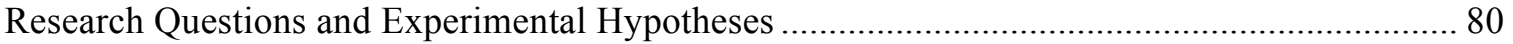

Manuscript I: Chronicity of Quadriceps Function in ACL Reconstructed Patients With and Without Knee Osteoarthritis.................................................................. 80

Manuscript II: Relationship Between Quadriceps Function and Patient Reported Outcomes in ACL Reconstructed Patients With and Without Knee Osteoarthritis.......... 81

Manuscript III: Quadriceps and Patient Reported Function in ACL Reconstructed Patients: A Principal Component Analysis................................................................ 82

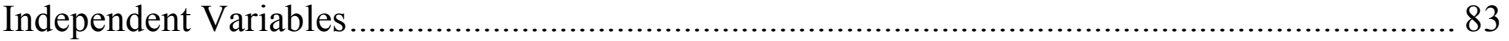

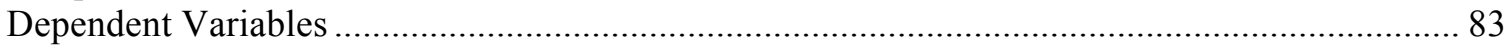

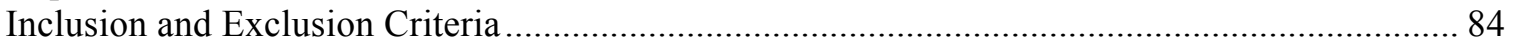

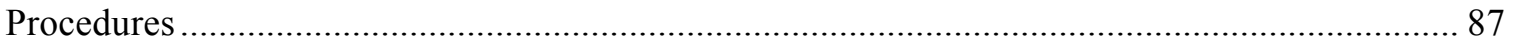

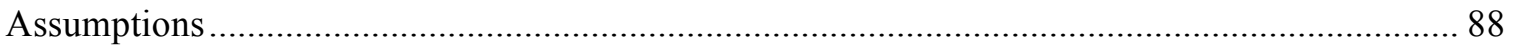

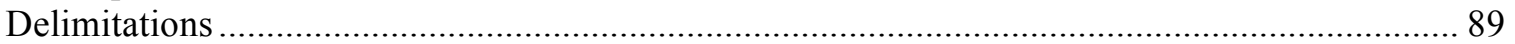

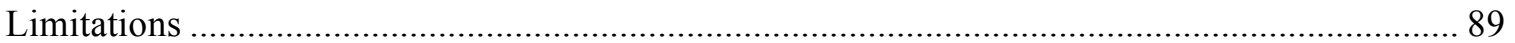

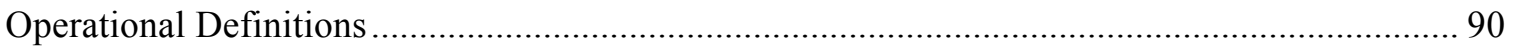

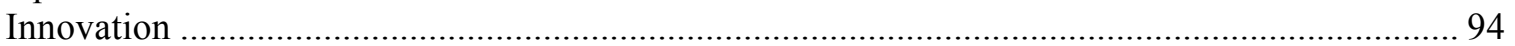

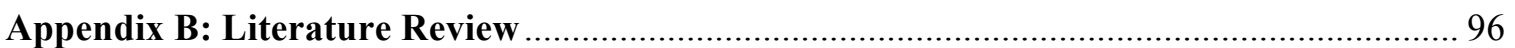

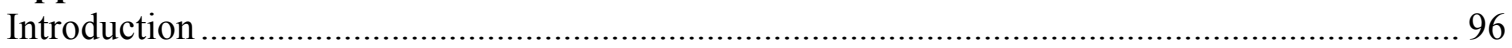

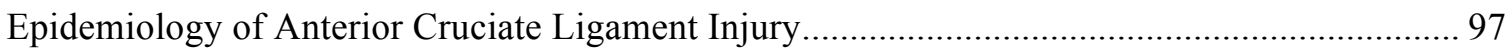

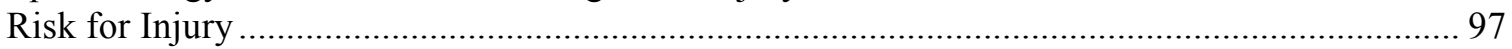

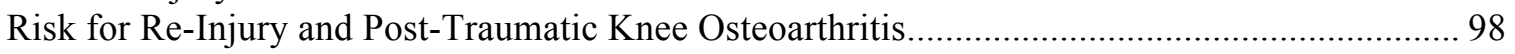

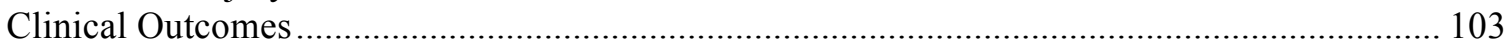

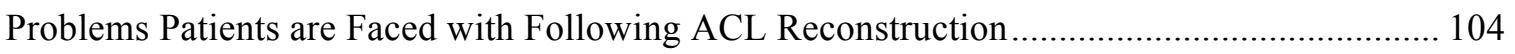

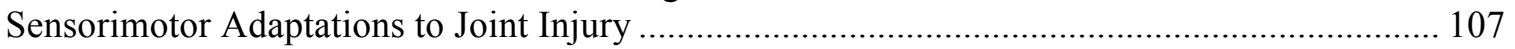

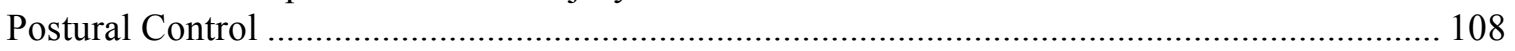

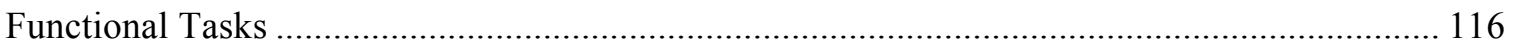

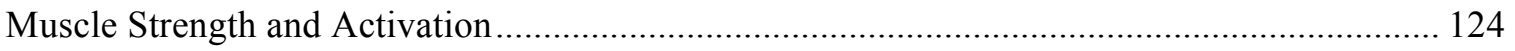

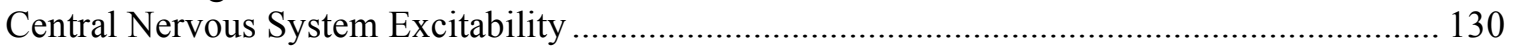

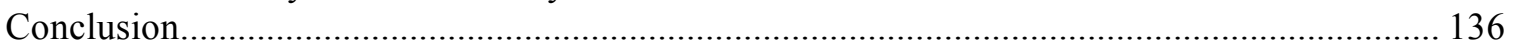

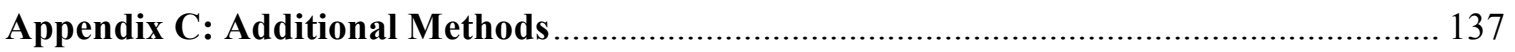

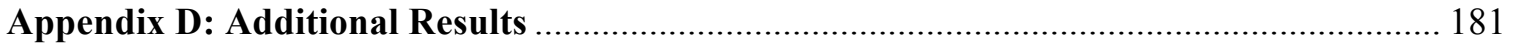

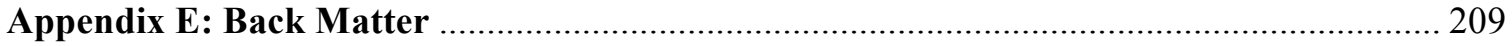

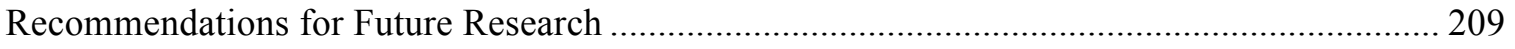

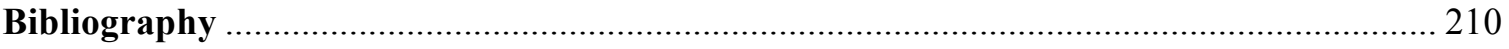




\section{LIST OF TABLES}

\section{MANUSCRIPT I}

Table $1_{\mathrm{i}}$. Participant demographics and patient reported outcomes ......................................... 20

Table $2_{\mathrm{i}}$. Quadriceps neuromuscular function in ACL-R patients and healthy individuals........... 21

\section{MANUSCRIPT II}

Table $1_{\text {ii }}$ Patient demographics.

Table $2_{\text {ii }}$. Association between quadriceps function, patient demographics, and

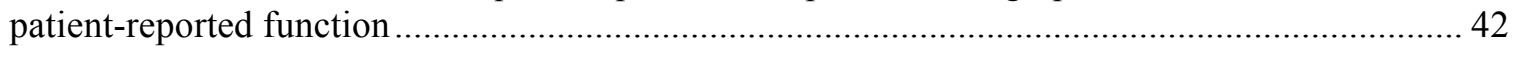

Table $3_{\mathrm{ii}}$. Final regression model in patients early after ACL-R............................................ 43

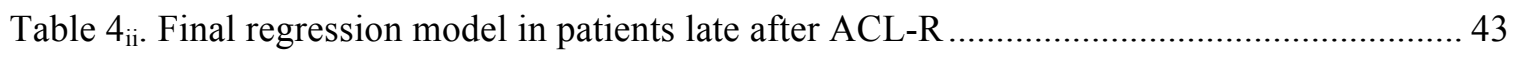

Table $5_{\text {ii }}$. Final regression model in patients with knee osteoarthritis after ACL-R .................... 43

\section{MANUSCRIPT III}

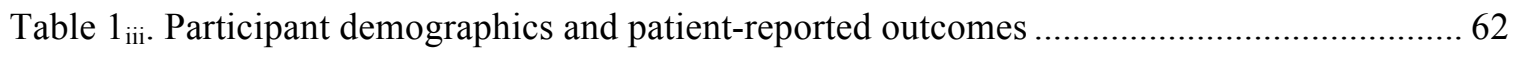

Table $2_{\text {iii }}$ Quadriceps neuromuscular function in ACL-R patients and healthy individuals ......... 63

Table $3_{\text {iii. }}$ Principal component analysis using involved limb measures of quadriceps

function

Table $4_{\text {iii. }}$ Principal component analysis using uninvolved limb measures of quadriceps

function

Table $5_{\text {iii. }}$ Principal component analysis using limb symmetry measures of quadriceps

function 66

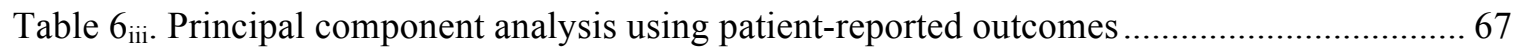

Table $7_{\text {iii. }}$ Results from logistic regression analyses to predict group membership ..................... 68

\section{APPENDIX C}

Table C1. Informed Consent Form 
Table C2. Transcranial Magnetic Stimulation Screening Form

Table C3. International Knee Documentation Committee (IKDC) Subjective Knee

Evaluation

Table C4. Knee Injury and Osteoarthritis Outcome Score (KOOS) ......................................... 142

Table C5. Visual Analog Scale (VAS) for Pain at Rest.......................................................... 143

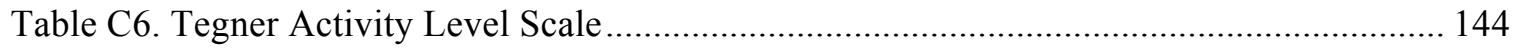

Table C7. Godin Leisure-Time Exercise Questionnaire ...................................................... 145

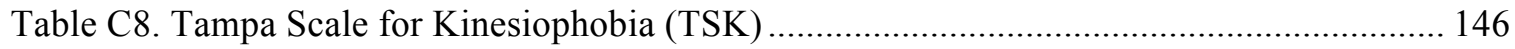

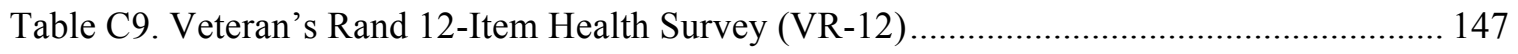

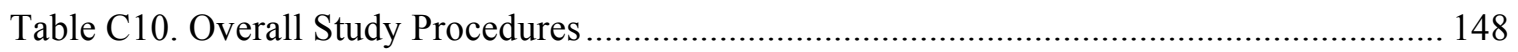

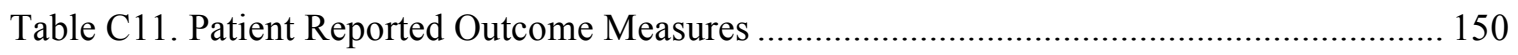

Table C12. Quadriceps Hoffmann Reflex Setup and Procedures ........................................... 151

Table C13. Quadriceps Isokinetic Torque Setup and Procedures ............................................. 155

Table C14. Quadriceps Superimposed Burst Technique Setup and Procedures......................... 158

Table C15. Quadriceps Fatigue Index Setup and Procedures .................................................. 161

Table C16. Transcranial Magnetic Stimulation Setup and Procedures..................................... 175

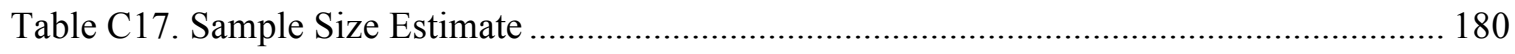

\section{APPENDIX D}

Table D1. ANOVA comparison of group and limb for normalized knee extension MVIC torque

Table D2. Post hoc analyses from ANOVA comparison of group and limb for normalized knee extension MVIC torque

Table D3. Between limb comparisons of normalized knee extension MVIC torque

Table D4. ANOVA comparison of group and limb for quadriceps fatigue index

Table D5. Post hoc analyses from ANOVA comparison of group and limb for quadriceps

fatigue index

Table D6. Between limb comparisons of quadriceps fatigue index.

Table D7. ANOVA comparison of group and limb for quadriceps central activation ratio 
Table D8. Post hoc analyses from ANOVA comparison of group and limb for quadriceps central activation ratio

Table D9. Between limb comparisons of quadriceps fatigue index

Table D10. ANOVA comparison of group and limb for normalized quadriceps Hoffmann reflex

Table D11. Post hoc analyses from ANOVA comparison of group and limb for normalized quadriceps Hoffmann reflex

Table D12. Between limb comparisons of normalized quadriceps Hoffmann reflex. 185

Table D13. ANOVA comparison of group and limb for quadriceps active motor threshold

Table D14. Post hoc analyses from ANOVA comparison of group and limb for quadriceps active motor threshold

Table D15. Between limb comparisons of quadriceps active motor threshold

Table D16. Multiple regression model summary to predict knee function (KOOS) in ACL reconstructed patients per group

Table D17. ANOVA to predict knee function (KOOS) in ACL reconstructed patients

Table D18. Multiple regression coefficients to predict knee function (KOOS) in ACL reconstructed patients

Table D19. Multiple regression model summary to predict global health (VR-12) in ACL reconstructed patients per group

Table D20. ANOVA to predict global health (VR-12) in ACL reconstructed patients

Table D21. Multiple regression coefficients to predict global health (VR-12) in ACL reconstructed patients

Table D22. Paired $t$ tests comparing the involved to uninvolved limb among ACL reconstructed patients

Table D23. Paired $t$ tests comparing the matched 'involved' to matched 'uninvolved' limb among healthy controls

Table D24. Independent $t$ tests comparing the involved ACL reconstructed limb to matched healthy limb

Table D25. Independent $t$ tests comparing the uninvolved ACL reconstructed limb to matched healthy limb

Table D26. Independent $t$ tests comparing limb symmetry indices between ACL reconstructed patients and healthy controls 
Table D27. Total variance explained by measures of involved ACL reconstructed limb quadriceps function

Table D28. Component and rotated component matrices for involved ACL reconstructed limb quadriceps function.

Table D29. Total variance explained by measures of uninvolved ACL reconstructed limb quadriceps function

Table D30. Component and rotated component matrices for uninvolved ACL reconstructed limb quadriceps function.

Table D31. Total variance explained by limb symmetry measures of quadriceps function in ACL reconstructed patients

Table D32. Component and rotated component matrices for limb symmetry measures of quadriceps function in ACL reconstructed patients

Table D33. Area under the receiver operator characteristic (ROC) curve for measures of involved limb quadriceps function to determine group membership.

Table D34. Area under the receiver operator characteristic (ROC) curve for measures of uninvolved limb quadriceps function to determine group membership.....

Table D35. Area under the receiver operator characteristic (ROC) curve for limb symmetry measures of quadriceps function to determine group membership

Table D36. Binary logistic regression results to predict group membership using measures of involved limb quadriceps function

Table D37. Binary logistic regression results to predict group membership using measures of uninvolved limb quadriceps function

Table D38. Binary logistic regression results to predict group membership using limb symmetry measures of quadriceps function 


\section{LIST OF FIGURES}

\section{MANUSCRIPT I}

Figure $1_{i}$. Group means with standard deviations (error bars) for involved and uninvolved limb quadriceps function.

Figure $2_{\text {i }}$. Cohen's $d$ effect sizes with $95 \%$ confidence intervals for involved limb quadriceps function compared to matched healthy controls

Figure $3_{\mathrm{i}}$. Relationship between normalized knee extension MVIC torque and quadriceps central activation ratio for the involved and uninvolved limbs.

\section{MANUSCRIPT II}

Figure $1_{\text {iI }}$. Relationship between self-reported knee function and fear of movement, and between self-reported global health and current activity level

\section{APPENDIX A}

Figure A1. Theoretical model depicting the temporal relationship between quadriceps function and clinical outcomes following ACL reconstruction

Figure A2. Summary of studies investigating quadriceps neuromuscular function indicating

time from ACL reconstruction

Figure A3. Clinically relevant time points after ACL reconstruction.....

\section{APPENDIX C}

Figure C1. Study flow chart

Figure C2. Quadriceps Hoffmann reflex testing setup and data processing....

Figure C3. Quadriceps isokinetic torque testing setup and data processing....

Figure C4. Quadriceps superimposed burst technique testing setup and data processing..... 160

Figure C5. Quadriceps fatigue index testing setup and data processing.... 174

Figure C6. Transcranial magnetic stimulation testing setup and data processing 179 


\section{APPENDIX D}

Figure D1. Quadriceps function limb symmetry (mean $\pm 95 \%$ confidence interval) for each group

Figure D2. Cohen's $d$ effect sizes with 95\% confidence intervals comparing subjective outcomes in each ACL reconstructed group to healthy controls

Figure D3. Pearson's $r$ or Spearman's $\rho$ correlation coefficients between measures of involved limb quadriceps function and time since surgery in ACL reconstructed patients.

Figure D4. Pearson's $r$ or Spearman's $\rho$ correlation coefficients between measures of involved limb quadriceps function and age in ACL reconstructed patients

Figure D5. Independent $t$ tests comparing measures of involved limb quadriceps function between male and female ACL reconstructed patients

Figure D6. ANOVA comparison of graft type for measures of involved limb quadriceps function in ACL reconstructed patients

Figure D7. Pearson's $r$ correlation coefficients between total KOOS score and pain at rest, kinesiophobia, and activity level in patients early after ACL reconstruction

Figure D8. Pearson's $r$ correlation coefficients between total VR-12 score and activity level, pain at rest, and time since surgery in patients early after ACL reconstruction

Figure D9. Pearson's $r$ correlation coefficients between total KOOS score and age in patients late after ACL reconstruction

Figure D10. Normalized knee extension MVIC torque for the involved and uninvolved limbs

Figure D11. Receiver operator characteristic (ROC) curves using measures of involved limb quadriceps function to determine group membership.

Figure D12. Receiver operator characteristic (ROC) curves using measures of uninvolved limb quadriceps function to determine group membership.

Figure D13. Receiver operator characteristic (ROC) curves using limb symmetry measures

of quadriceps function to determine group membership. 206 
SECTION II: MANUSCRIPT I

CHRONICITY OF QUADRICEPS FUNCTION IN ACL RECONSTRUCTED PATIENTS WITH AND WITHOUT KNEE OSTEOARTHRITIS 


\begin{abstract}
Context: Central and peripheral neural adaptations from muscular, spinal, and supraspinal regions have been identified following ACL injury and reconstruction and are hypothesized to contribute to post-traumatic muscle dysfunction. Currently, there is limited evidence with regard to the temporal nature of neuromuscular adaptions during early and late term durations after ACL-R, and none that include patients with post-traumatic osteoarthritis. Objective: To compare quadriceps neuromuscular function early and late after ACL-R, including patients who experienced post-traumatic knee osteoarthritis. Design: Cross sectional. Setting: University laboratory. Patients or Participants: 102 participants volunteered for this study, including 72 ACL reconstructed patients: Early $(\mathrm{n}=34,20 \mathrm{M} / 14 \mathrm{~F}, 9.0 \pm 4.3$ months post-ACL-R), Late $(\mathrm{n}=$ 30, 10M/20F, $70.5 \pm 41.6$ months post-ACL-R), Osteoarthritis $(\mathrm{n}=8,2 \mathrm{M} / 6 \mathrm{~F}, 115.9 \pm 110.0$ months post-ACL-R), and 30 healthy individuals (12M/18F). Intervention(s): None. Main Outcome Measure(s): Normalized knee extension maximum voluntary isometric contraction (MVIC) torque ( $\mathrm{Nm} / \mathrm{kg}$ ), quadriceps fatigue index (\% decline), quadriceps central activation ratio (CAR, \%), quadriceps spinal reflex excitability (H:M ratio), quadriceps corticospinal excitability (AMT, \% 2.0 Tesla) were measured bilaterally. Comparisons were made using two-way analyses of variance to determine the effect of limb and group on MVIC torque, fatigue index, CAR, H:M ratio, and AMT. Results: Compared to the healthy control group, MVIC torque was lower among all ACL-R patients $(p<.001)$, quadriceps fatigue index $(p=.003)$ and CAR $(p<.001)$ were lower among early ACL-R patients only, and quadriceps AMT was higher among all ACL-R patients $(p<.001)$. Conclusions: Neuromuscular impairments are present in patients early and late after ACL-R with and without knee osteoarthritis. Quadriceps strength and corticospinal excitability were impaired at all time points compared to healthy individuals, suggesting the role of addressing cortical function following ACL-R.
\end{abstract}

Word Count: 289

Key Words: corticospinal, neuromuscular, quadriceps activation 


\section{INTRODUCTION}

Anterior cruciate ligament (ACL) injuries are common among young, active individuals, ${ }^{1}$ and present a specific challenge to long-term joint health. ${ }^{2}$ ACL reconstruction (ACL-R) remains the recommended treatment in this population; ${ }^{3}$ unfortunately, poor outcomes are well documented. ${ }^{4}$ As many as one-third of patients will not return to pre-injured levels of activity, ${ }^{5}$ and among those who do, prospective data supports the dramatically increased incidence of a second ACL injury to the ipsilateral or contralateral limb within two years of reconstruction. ${ }^{6}$ More concerning is the high prevalence of post-traumatic knee joint osteoarthritis, with radiographic signs appearing as early as the first decade in more than one-third of patients following reconstruction. ${ }^{4}$ Quadriceps function has been widely studied in response to ACL-R, both as a source of persistent impairment and contributing factor for subsequent joint injury. ${ }^{7}$ Post-traumatic quadriceps dysfunction is well described following ACL-R, ${ }^{8}$ and is associated with decreased physical activity ${ }^{9}$ and increased self-reported global and regional disability. ${ }^{10}$ Moreover, quadriceps weakness is reported to be associated with diminished tibiofemoral joint space width, ${ }^{11}$ which may contribute to the progression of osteoarthritis. ${ }^{12}$ Since articular cartilage degeneration is irreversible, the hallmark for prevention is early detection with thorough evaluation of quadriceps neuromuscular function.

Central and peripheral neural adaptations from muscular, spinal, and supraspinal regions have been identified following ACL injury and reconstruction, ${ }^{13}$ and are hypothesized to contribute to post-traumatic muscle dysfunction. ${ }^{14}$ The specific origins of impairment have been theorized as a way to address subtle underlying factors impeding the recovery of quadriceps function following ACL-R. ${ }^{15}$ Central activation failure of the quadriceps has been identified more than four years following ACL- $\mathrm{R}^{13,16}$ and in patients with radiographic tibiofemoral osteoarthritis. ${ }^{17}$ Neuromuscular adaptations are theorized to arise from alterations in spinalmediated and corticospinal pathways, ${ }^{18}$ and if unaddressed, may present a limiting factor in recovery from ACL injury. ${ }^{19}$ Therefore, it may be necessary to assess each of these unique 
pathways to build a complete neuromuscular profile following ACL-R in an attempt to identify early and subtle deficits that may lead to persistent muscle dysfunction.

Outcomes following major joint injury and reconstruction often deteriorate with time, suggesting that chronicity plays an important role in identifying those at risk for long term consequences associated with altered neuromuscular function. The nature and magnitude of neural adaptations following peripheral joint injury is reported to change over time. For example, alterations in spinal reflex excitability have been identified in response to a simulated knee joint effusion, ${ }^{20}$ suggesting an acute neural response to joint injury mediated at the spinal level. However, spinal-mediated alterations have not been consistently reported in the context of chronic ACL injury. ${ }^{13,16}$ For example, researchers have reported no differences in spinal reflex excitability at an average of 2.5 years ${ }^{16}$ following ACL-R compared to healthy individuals; whereas, others have observed a bilateral up-regulation at an average of 4 years post-op. ${ }^{13}$ In contrast, immediate changes in corticospinal excitability have not been observed in response to a simulated knee joint effusion; ${ }^{21}$ yet, deficits have been observed more than 3 years following ACL-R. ${ }^{13,16}$ These findings agree with a recent longitudinal study reporting decreased spinal reflex excitability prior to and 2 weeks following ACL-R, and a decrease in corticospinal excitability at 6 months following ACL-R ${ }^{15}$ Collectively, these data provide evidence of the temporal nature of the pathophysiological response to ACL injury and reconstruction, although long-term evaluation is warranted to understand the impact on clinical outcomes. Neuromuscular adaptations appear to be an expected outcome following joint injury. However, these deficits are treatable and present a way for clinicians to detect problems early with the intention of promoting optimal long-term joint health, especially in the prevention of early-onset knee osteoarthritis.

Currently, there is growing evidence of central nervous system adaptations following ACL-R $;^{13,22}$ however, the temporal relationship of these adaptations is unclear. It may be possible that a time point exists along the continuum of recovery in which patients with successful and poor outcomes diverge, indicating a critical junction in a targeted rehabilitation process. Previous 
models of study have largely classified ACLR patients as a single group of comparison relative to healthy counterparts with widespread time since surgery, which may prevent early detection of impairments or delay early intervention. In an effort to better understand how neuromuscular adaptations progress over time, time from surgery should be considered. There is limited evidence with regard to the specific timing of alterations in neuromuscular function of the quadriceps during early $(<1$ year) and late $(>2$ years) term durations after ACL-R, and none that include patients with post-traumatic osteoarthritis following ACL-R. Therefore, the purpose of this study was to compare quadriceps neuromuscular function at clinically relevant time points following ACL-R, including patients who experienced post-traumatic knee osteoarthritis.

\section{METHODS}

This was a cross-sectional study to investigate patients following ACL-R and healthy controls. Independent variables included one between factor (Group: < 1 year post ACL-R, > 2 years post ACL-R, patients with radiographic evidence of knee osteoarthritis post ACL-R, and healthy matched controls) and one within factor (Limb: involved, uninvolved). Dependent variables included measures of quadriceps neuromuscular function, including knee extension maximal voluntary isometric contraction (MVIC) torque, fatigue index (FI), central activation ratio (CAR), Hoffmann reflex (H-reflex), and active motor threshold (AMT). Quadriceps neuromuscular function was recorded bilaterally, and the order of testing was counterbalanced by limb for each group. Limb dominance was recorded for each participant, and determined by asking which limb would be used to kick a ball. The International Knee Documentation Committee (IKDC) subjective form, Knee Injury and Osteoarthritis Outcome Score (KOOS), and Western Ontario and McMaster Universities Arthritis Index (WOMAC) were used to measure regional patient-reported function.

\section{Participants}

A total of 102 subjects volunteered for this study, including 72 ACL reconstructed patients: < 1 year $(n=34),>2$ years $(n=30)$, osteoarthritis: 9.7 years (range, 10 to 301 months) 
$(\mathrm{n}=8)$, and 30 healthy controls between the ages of $15-65$. To be eligible, patients must have undergone a primary, unilateral reconstruction with no post-surgical complications.

Meniscectomy and meniscal repair were accepted as potential concomitant procedures. Patients were excluded if they had a history of lower extremity injury other than ACL-R within 6 months, multiligament knee injury, lower extremity orthopaedic surgery prior to ACL-R, or concussion within 6 months. Patients with knee osteoarthritis must have had documented radiographic evidence of tibiofemoral or patellofemoral compartment involvement (Kellgren-Lawrence > 1) at a minimum of 12 months post ACL-R. Patients with a diagnosis prior to ACL-R were excluded. A convenience sample of healthy individuals with no history of lower extremity injury within 6 months or prior joint surgery was recruited from the community. All participants were screened for the use of transcranial magnetic stimulation according to the safety and ethical guidelines proposed by the International Federation of Clinical Neurophysiology. ${ }^{23}$ Our University’s Institutional Review Board for Health Sciences Research approved this study, and all participants provided informed consent prior to enrollment.

\section{Procedures}

Testing was conducted during one study visit, which always followed the same order: Hreflex, knee extension MVIC torque, quadriceps CAR, FI, and AMT.

\section{Patient Reported Outcomes}

Regional knee function was assessed using the 2000 International Knee Documentation Committee (IKDC) subjective knee form ${ }^{24}$ and Knee Injury and Osteoarthritis Outcome Score (KOOS) ${ }^{25}$ The KOOS was used to supplement the IKDC in order to assess specific functional domains of pain, symptoms, activities of daily living, sport related function, and quality of life. The Western Ontario and McMaster Universities Arthritis Index (WOMAC) is advocated for use among patients with osteoarthritis, and was used to assess pain, stiffness, and function. ${ }^{26}$ The combination of measurement tools for regional knee function was used to ensure appropriate assessment for all patients over a long duration of time from ACL-R (range, 4.2 - 301.2 months). 
Activity level, fear of movement, and global health perception were quantified using the Tegner Activity Scale, ${ }^{27}$ Tampa Scale of Kinesiophobia (TSK), ${ }^{28}$ and Veteran's Rand 12-Item Health Survey (VR-12) ${ }^{29}$ respectively.

\section{Quadriceps Spinal Reflex Excitability}

The H-reflex was used to quantify spinal reflex excitability of the quadriceps as previously described. ${ }^{30}$ The area of the greatest bulk over the vastus medialis was cleaned, shaved, and debrided, prior to placement of two $10 \mathrm{~mm}$ pre-gelled $\mathrm{Ag}-\mathrm{AgCl}$ recording electrodes (EL503, BIOPAC Systems, Inc.) in accordance with surface electromyography (EMG) for noninvasive assessment of muscle guidelines. ${ }^{31}$ EMG signals were sampled at $2000 \mathrm{~Hz}$, bandpass filtered from 10 to $500 \mathrm{~Hz}$, and processed using AcqKnowledge 4.2 software (BIOPAC Systems, Inc., Goleta, CA). The recording electrodes were outlined to ensure similar placement during corticospinal testing. A ground electrode was placed over the contralateral distal anteromedial tibia. Testing was completed in a quiet, dimly lit room in which participants lied supine with a bolster under the knees to maintain approximately $15^{\circ}$ of knee flexion, and hands folded over their chest. Participants wore earplugs (Aero Technologies, Indianapolis, IN) and were instructed to "close your eyes, and clear your mind" during testing. The femoral artery was palpated, and a $4 \mathrm{~mm}$ rounded stimulating electrode (EL254, BIOPAC Systems, Inc.) was placed in the inguinal fold over the femoral nerve. A round carbon dispersive electrode was placed under the ipsilateral posterior thigh. A series of $10 \mathrm{~ms}$ square wave electrical stimuli ranging 10-200 volts were delivered via stimulator module (STM100A, BIOPAC Systems, Inc.) and current isolation unit (STMISOC, BIOPAC Systems, Inc.) with a minimum of 10 seconds between stimuli. Three maximal Hoffmann reflexes were averaged and normalized to the average of three maximal muscle responses (M-response) to calculate the H:M ratio. ${ }^{30,32}$ 


\section{Quadriceps Strength and Voluntary Activation}

Knee extension MVIC torque and the quadriceps CAR were used to quantify quadriceps strength and voluntary activation. Participants were seated in a Biodex System 3 Dynamometer (Biodex Medical Systems, Inc., Shirley, NY) with the hips and knees flexed to $85^{\circ}$ and $90^{\circ}$ respectively. Participants completed a standardized acclimatization protocol, in which a series of submaximal trials $(25 \%, 50 \%, 75 \%$ perceived effort) were performed prior to recording three

maximal effort trials. ${ }^{30}$ Accessory motion was discouraged by having participants cross their arms over their chest, while keeping their head and shoulders back against the rest. To ensure maximal effort, verbal encouragement was provided. Participants were provided with visual feedback via 50-inch LCD monitor, and encouraged to kick harder than each previous trial. Participants rested for a minimum of 60 seconds between trials.

The superimposed burst technique was used as previously described to estimate quadriceps CAR during the third maximal effort trial. ${ }^{33}$ A square wave stimulator (S88, GRASSTeleFactor, W. Warwick, RI) and isolation unit (SIU8T, GRASS-TeleFactor) were used to deliver a supramaximal percutaneous electrical stimulus to the anterior thigh via two $3 \times 5$ in selfadhesive electrodes applied over the proximal vastus lateralis and distal vastus medialis. Force data were sampled at $125 \mathrm{~Hz}$, low-pass filtered at $10 \mathrm{~Hz}$, and normalized to body mass. A $100 \mathrm{~ms}$ epoch was recorded from a stable region of trials 1 and 2, and from immediately prior to the superimposed burst torque of trial 3. The mean MVIC torque from the three MVIC trials was used for analysis, and the MVIC torque from trial 3 was used to calculate the CAR as previously described. ${ }^{34}$

\section{Quadriceps Fatigue Index}

Quadriceps fatigue was quantified using a previously described ${ }^{35}$ index of torque decline during a 30 second knee extension MVIC. Participants were instructed to kick out as hard as possible, and to maintain the contraction while seated in a Biodex System 3 Dynamometer in a similar fashion to quadriceps strength testing. Participants were prompted to start kicking, 
increasing to a maximal effort over three seconds. The 30-second trial began after three seconds, once the participant had achieved their perceived maximal effort. Verbal encouragement and visual feedback were not used to minimize the occurrence of transient aberrant increases in torque, which were observed during pilot testing. Force data were sampled at $125 \mathrm{~Hz}$, low-pass filtered at $15 \mathrm{~Hz}$, and processed using Acqknowledge software. The mean torque was recorded from a series of 1-second epochs, and the greatest torque epoch during the first 5 seconds of the trial was recorded as the maximal torque $\left(\mathrm{T}_{\mathrm{Max}}\right)$. Quadriceps FI was calculated using the area under the force-time curve (AUFC) for the entire contraction period for 0 to 30 seconds, which began at the time point of maximum muscle torque (TPM) (Equation 1).

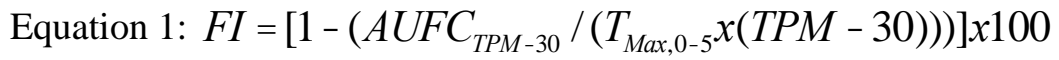

\section{Quadriceps Corticospinal Excitability}

Transcranial magnetic stimulation (TMS) was used to quantify quadriceps corticospinal excitability as measured by the AMT. Participants were asked to abstain from caffeine consumption and intense exercise for a minimum of 12 hours prior to testing. Participants were seated in a Biodex System 3 Dynamometer, and asked to rest their hands in their lap while minimizing accessory motion. Two surface EMG electrodes were replaced over the vastus medialis in a similar manner as the Hoffmann reflex testing. Participants wore earplugs and a Lycra swim cap with straight lines drawn vertically in the sagittal and frontal planes to aid in determining the appropriate location for the TMS coil over the primary motor cortex (M1). ${ }^{36}$ A 1 $\mathrm{cm} \times 1 \mathrm{~cm}$ grid was drawn on the swim cap to improve the precision of stimuli delivered. Motor evoked potentials (MEP) were elicited in the vastus medialis using a magnetic stimulator (Magstim Rapid, MagStim Company, Ltd., Wales, UK) with a $110 \mathrm{~mm}$ double-cone coil. MEP signals were sampled at $2000 \mathrm{~Hz}$, and band-pass filtered from 1 to $5000 \mathrm{~Hz}$. The coil was positioned over the contralateral cortical hemisphere in the area of M1, and shifted by $0.5 \mathrm{~cm}$ in the anterior, posterior, medial, and lateral directions to identify the optimal stimulating location 
(hotspot). ${ }^{37}$ The hotspot was defined as the greatest MEP amplitude. A researcher manually positioned the coil for each measurement while standing behind the participant on a $54 \mathrm{~cm}$ step. Once the hotspot was determined, the stimulus intensity was sequentially lowered by $5 \%$ until no MEP was detected, then increased by $1 \%$ until the MEP returned. The AMT was defined as the lowest intensity required to evoke a measurable MEP $(>200 \mu \mathrm{V})$ during a tonic contraction. ${ }^{23}$ Participants were instructed to maintain an isometric contraction at 5\% of their previously determined MVIC, and to relax immediately following each stimulus. ${ }^{16}$ Participants were instructed to match solid line depicting 5\% of the MVIC that was displayed in real time on an LCD monitor, and to relax between each stimulus.

\section{Statistical Analysis}

A sample size estimate was based on the minimal detectable change $\left(\mathrm{MDC}_{95}\right)$ for each dependent variable, assuming an alpha level of $p \leq .05$ and power of $1-\beta=0.80$. The $\mathrm{MDC}_{95}$ was calculated as $\sqrt{2} \times 1.96 \times$ SEM. Accordingly, the $\mathrm{MDC}_{95}$ and required sample per group for each dependent variable were: $\operatorname{MVIC}^{38}(47.8 \mathrm{Nm}, \mathrm{n}=24), \mathrm{CAR}^{38}(6.0 \%, \mathrm{n}=16)$, fatigue index ${ }^{39}$ $(11.0 \%, \mathrm{n}=11), \mathrm{H}: \mathrm{Mratio}^{40}(0.30, \mathrm{n}=11)$, and $\mathrm{AMT}^{41}(8.4 \%, \mathrm{n}=14)$.

All data were assessed for normality prior to analysis. Separate one-factor analyses of variance were used to compare demographics and patient reported outcomes between groups (4 levels). Separate mixed model 2 x 4 (limb x group) analyses of variance were used to assess differences between the involved and uninvolved limbs for each measure of quadriceps function across all groups. In healthy participants, the non-dominant limb was initially treated as the “involved limb.” However, 57\% of ACL-R limbs were dominant. Therefore, a random number generator was used to re-assign an equal proportion of dominant to non-dominant limbs in the healthy group compared to ACL-R patients (i.e. the dominant limb was now treated as the "involved limb" in 57\% of the participants in each group). Separate one-factor analyses of variance were performed to compare groups (at 4 levels) for each limb in the event of significant main effects where appropriate. Dunnett's post-hoc comparisons were used to compare each 
ACL-R group to the healthy control group. Fisher's LSD post-hoc comparisons were used to determine group differences among ACL-R patients only. Planned comparisons between limbs were made using paired sample $t$-tests. Cohen's $d$ effect sizes with associated $95 \%$ confidence intervals were calculated to determine the magnitude of difference between the involved limb in ACL-R patients and healthy individuals for each outcome measure. Effect sizes with confidence intervals that did not cross zero were considered clinically meaningful.

As an exploratory analysis, bivariate Pearson's product moment correlation coefficients ( $r$ ) were used to assess the relationship between knee extensor MVIC torque and quadriceps CAR in each limb between groups. The level of statistical significance was set a priori at $P \leq .05$. All statistical analyses were performed using SPSS (version 20.0, IBM, Chicago, IL).

\section{RESULTS}

Group demographics and patient-reported function data are presented in table 1. Age was significantly different between groups $\left(F_{3,97}=36.1, p<.001\right)$, indicating that patients with osteoarthritis were older than all other participants $(p<.001)$. Group means and standard deviations are presented for MVIC torque, FI, CAR, H-reflex, and AMT (Table 2, Figure 1). Effect sizes demonstrating the magnitude of difference between the involved ACL-R and matched healthy limbs are presented for each dependent variable in figure 2.

\section{Patient Reported Outcomes}

Each ACL-R group reported significantly lower knee function compared to healthy controls (group main effect for IKDC, KOOS, and WOMAC, $p<.001$ ). IKDC and WOMAC scores were higher in ACL-R patients without osteoarthritis compared to those with osteoarthritis, but did not differ between early and late ACL-R groups. Among ACL-R patients, those early after ACL-R reported the highest KOOS scores, followed by the late and osteoarthritis groups.

\section{Quadriceps Spinal Reflex Excitability}

$\mathrm{H}: \mathrm{M}$ ratio was not significantly different by group $\left(F_{3,176}=2.5, p=.065\right)$ or $\operatorname{limb}\left(F_{1,176}=\right.$ $0.01, p=.916)$. Between limb differences were not detected for any group. Effects sizes were 
moderate for early $(d=0.45[-0.03,0.94])$ and late $(d=0.59[0.09,1.09])$ ACL-R patients, and large for patients with osteoarthritis $(d=0.98[0.09,1.87])$.

\section{Quadriceps Strength and Voluntary Activation}

A significant group main effect $\left(F_{3,194}=11.6, p<.001\right)$ indicated that each ACL-R group demonstrated lower knee extension MVIC torque than healthy controls. A significant limb main effect $\left(F_{1,194}=5.5, p=.02\right)$ indicated that the involved ACL-R limb was weaker in patients early $(d=-1.46[-2.01,-0.91])$, late $(d=-1.00[-1.54,-0.46])$, and with osteoarthritis $(d=-1.75[-2.62,-$ 0.88]) compared to healthy controls; however, there were no differences between ACL-R patients. Uninvolved limb MVIC torque was lower in the late ACL-R and osteoarthritis groups compared to healthy controls. Patients with osteoarthritis were also weaker in the uninvolved limb compared to patients early after ACL-R. A significant group-by-limb interaction $\left(F_{3,194}=\right.$ $4.8, p=.003$ ) indicated that the involved limb was weaker than the uninvolved limb in the early and late ACL-R groups $(p=.029)$, but not in patients with osteoarthritis. There was no difference between limbs in the healthy control group.

A significant group main effect $\left(F_{3,193}=6.8, p<.001\right)$ indicated that quadriceps CAR was lower early $(d=-1.74[-2.32,-1.16])$, but not late $(d=-0.84[-1.37,-0.31])$ after ACL-R, or in patients with osteoarthritis $(d=-0.45[-1.24,0.33])$, compared to healthy controls. CAR was significantly lower in the early compared to late and osteoarthritis groups. CAR did not differ significantly by $\operatorname{limb}\left(F_{1,193}=0.1, p=.711\right)$ or group-by-limb $\left(F_{3,193}=0.4, p=.781\right)$. There were no differences between limbs for any group.

\section{Quadriceps Fatigue Index}

A significant group main effect $\left(F_{3,193}=4.9, p=.003\right)$ indicated that patients in the early ACL-R group fatigued less than patients in the late ACL-R group and healthy controls $(d=-0.95$ $[-1.47,-0.43])$. Quadriceps fatigue was not different between the early ACL-R and osteoarthritis groups. Fatigue index did not differ by $\operatorname{limb}\left(F_{1,193}=0.2, p=.619\right)$ or group-by-limb $\left(F_{3,193}=1.8\right.$, $p=.147)$. Planned comparisons between limbs revealed that patients in the early ACL-R group 
fatigued significantly less in the involved compared to the uninvolved limb $(p=.002)$. There were no differences between limbs for any other group.

\section{Quadriceps Corticospinal Excitability}

A significant group main effect $\left(F_{3,173}=9.7, p<.001\right)$, indicated AMT was higher bilaterally in patients early $(d=-0.87[-1.44,-0.30])$, late $(d=-0.42[-1.00,0.15])$, and with osteoarthritis $(d=-1.56[-2.46,-0.65])$ compared to healthy controls (decreased corticospinal excitability). Patients in the early ACL-R and osteoarthritis groups had a higher AMT compared to the late ACL-R group. AMT did not differ by limb $\left(F_{1,173}=1.0, p=.319\right)$ or group-by-limb $\left(F_{3,173}=0.2, p=.880\right)$. There were no differences between limbs for any group.

\section{Correlations}

Involved limb MVIC torque and CAR were correlated early $(r=.546, p=.001)$ and late $(r=.486, p=.006)$ after ACL-R, but not in patients with knee osteoarthritis $(r=-.135, p=.750)$. Uninvolved limb MVIC torque and CAR were correlated late after ACL-R $(r=.388, p=.034)$, but not early $(r=.200, p=.257)$, or in patients with knee osteoarthritis $(r=-.020, p=.963)$.

\section{DISCUSSION}

Our results indicate that patients early after ACL-R demonstrated reduced quadriceps strength in the involved limb, and bilateral reductions in fatigue, voluntary activation, and corticospinal excitability compared to healthy controls. Less symmetric quadriceps strength and fatigue indices were also observed in patients early. Patients with and without knee osteoarthritis late after ACL-R demonstrated a bilateral reduction in quadriceps strength and corticospinal excitability compared to healthy controls. Less symmetric quadriceps strength was also observed in patients late after ACL-R, but not in those with knee osteoarthritis. This study was the first to cross-sectionally examine quadriceps neuromuscular function at distinct early and late phases of ACL-R recovery with the inclusion of a post-traumatic osteoarthritic cohort. These data provide supporting evidence of the temporal nature of neuromuscular adaptations after ACL-R. 


\section{Early Post-Operative Outcomes}

Large magnitude reductions in unilateral quadriceps strength, fatigue, voluntary activation, and corticospinal excitability were observed between 6 and 12 months post ACL-R compared to healthy controls, which is supported by large and clinically meaningful effect sizes. H:M ratio did not statistically differ from healthy limbs; however, a moderate effect size was calculated for the involved limb, suggesting a clinically meaningful up-regulation of spinal reflex excitability. These findings agree with longitudinal data of neural function in patients 6 months after ACL-R. ${ }^{15}$ In this study, spinal reflex excitability increased from 2 weeks to 6 months post ACL-R despite not being different from healthy controls. In contrast, corticospinal excitability did not differ from healthy controls by 2 weeks, but was significantly reduced by 6 months after ACL-R. These data suggest a temporal, yet reciprocal relationship between spinal-mediated and cortically driven signaling to muscle, which supports the theoretical attempt to maintain muscle function. The time frame in which neural adaptations manifest is unclear.

Persistent quadriceps weakness is reported to manifest from aberrant sensory information arising from damaged peri-articular tissue. ${ }^{42}$ Early after ACL injury and reconstruction, the presence of pain, swelling, and inflammation may stimulate nociceptors and articular mechanoreceptors, theorized to result in an ongoing reflexive inhibition of uninjured musculature due to a net reduction in spinal-mediated excitability. ${ }^{14}$ Clinical signs of swelling and inflammation were not observed in our cohort, and only minimal pain was reported $(<1 / 10 \mathrm{~cm})$. Previous authors ${ }^{43,44}$ have used rabbit models to investigate the ongoing influence of ACL injury on sensory input to the central nervous system. In one study, ${ }^{44}$ the ACL was transected with or without patellar tendon reconstruction, and sensory information arising from the femoral nerve was measured. After two weeks, a large magnitude $(d=1.15[0.54,1.76])$ and statistically significant increase in afference of the ACL reconstructed limb was observed compared to control rabbits, suggesting that overload of sensory information transmission may contribute to persistent muscle dysfunction after ACL-R. Continued alterations in sensory integration after ACL-R 
despite the resolution of pain and swelling may have contributed to the persistent muscle dysfunction observed in this study. Although spinal reflex excitability was not statistically different from healthy individuals in our study, a clinically meaningful up-regulation was detected, which may have began to occur as clinical signs of injury resolved. If true, this may suggest that persistent sensory aberrations inhibit peripheral musculature via supraspinal, and not only spinal-mediated mechanisms. In support of this, we observed a decrease in corticospinal excitability at each time point after ACL-R, suggesting that alterations in the threshold of neuronal depolarization in the motor cortex may be a mediating factor, contributing to the observed reduction in quadriceps strength and voluntary activation.

Interestingly, the quadriceps fatigued less than healthy control limbs early after ACL-R. Reduced knee extensor fatigue has been observed in patients with quadriceps dysfunction (CAR $<90 \%$ ) more than 6 months post ACL-R compared to healthy counterparts following 30 minutes of continuous exercise. ${ }^{45}$ Previous authors have hypothesized that selective type II fiber atrophy of the injured limb may explain this phenomenon, supporting the role of morphological adaptations in muscle dysfunction after ACL-R. ${ }^{46}$ Type II atrophy of the quadriceps has been observed in patients 13 months post ACL-R, ${ }^{47}$ which may be an attempt to maintain function during activity, although this finding has been inconsistently reported. ${ }^{48,49}$ Muscle atrophy and fiber type were not assessed in the current study. However, atrophy of type II fibers is likely to occur early after ACL injury due to disuse, which may persist through the first year of recovery and explain the observed reduction in quadriceps strength and activation. In contrast, an increase in quadriceps fatigue was observed by two years compared to the early group, which did not differ from healthy controls, which may indicate a partial recovery of type II muscle fibers, if atrophied during early recovery; however, the presence of persistent weakness suggests incomplete morphological recovery. Chronic ACL injury may result in selective type $\mathrm{I}^{48}$ or a combination of type I and type II fiber atrophy, ${ }^{49}$ thereby explaining the long-term persistence of muscle weakness, despite an increase in fatigability. If patients are returning to more activity 
beyond the first year from surgery, type II fiber hypertrophy could possibly be explained. Muscle dysfunction during early recovery likely appears to be a product of combined neuromuscular and morphological factors.

\section{Late Post-Operative Outcomes}

Large magnitude reductions in quadriceps strength were observed bilaterally late after ACL-R, compared to healthy controls, which is supported by a large and clinically meaningful effect size calculated for the involved limb. Quadriceps CAR was significantly higher in patients late after ACL-R compared to early ( $90.4 \%$ vs. $85.5 \%)$, but not statistically different from healthy controls (95.2\%) or patients with knee osteoarthritis (92.6\%). This could be interpreted as an improvement in voluntary activation over time; however, a large and clinically meaningful effect size was calculated between the involved and matched healthy control limb, suggesting the presence of persistent central activation failure during late recovery beyond what is considered normal. A bilateral reduction in corticospinal excitability was observed compared to healthy individuals; however, the effect size was moderate and the confidence interval crossed zero. Interestingly, a pattern of up-regulated spinal reflex excitability was observed similar to patients early after ACL-R, which was supported by a moderate and clinically meaningful effect size, despite the absence of a statistical difference. The combination of a meaningful increase in spinal reflex and corticospinal excitability may have contributed to the improvement in voluntary activation observed late after ACL-R. Previous authors ${ }^{13}$ have identified increased spinal reflex excitability bilaterally at an average of 4 years after ACL-R, which is theorized to be a shunting response to maintain voluntary activation. This could explain the improvement of activation observed, yet may still be inadequate to fully restore quadriceps strength.

\section{Knee Osteoarthritis}

Patients with knee osteoarthritis demonstrated reductions in quadriceps strength and corticospinal excitability bilaterally, with large and clinically meaningful effect sizes calculated for the involved limb compared to healthy controls. Quadriceps strength did not differ among 
ACL-R patients at any time point in the involved limb, which may reflect an ongoing inhibitory process preventing complete recovery of quadriceps function. Interestingly, both the involved and contralateral limb was weaker in patients with knee osteoarthritis compared to all other ACL-R patients including healthy individuals. The reduction in contralateral limb strength likely contributed to the symmetry observed in this patient group, and may provide a false sense of successful outcomes when based on symmetry alone.

The relationship between quadriceps strength and voluntary activation is hypothesized to have a greater association early after ACL-R,${ }^{50}$ which is not present at $2-15$ years. ${ }^{51}$ Our data partially support this in that involved limb MVIC torque and CAR were correlated early and late after ACL-R, but not in patients with knee osteoarthritis (figure 3). Patients with knee osteoarthritis demonstrated a bilateral reduction in MVIC torque compared to healthy controls; yet fell within normal limits of healthy voluntary activation. This divergence between quadriceps strength and voluntary activation may suggest that a point along the spectrum from late recovery to the onset of osteoarthritis exists where patients must adapt to use a larger proportion of an already diminished motor neuron pool. This could explain why patients with knee osteoarthritis exhibit relatively high activation despite being significantly weaker than healthy individuals. Patient with knee osteoarthritis also demonstrated the lowest corticospinal excitability coupled with the highest spinal reflex excitability (not statistically significant) compared to healthy individuals, each of large magnitude and clinically meaningful differences. It would appear that a reorganization of the central nervous system may occur in patients with chronic joint degeneration after ACL-R in an effort to maintain adequate muscle activation. A differentiation in organization of the motor cortex has been identified in patients with and without knee osteoarthritis, ${ }^{52}$ further highlighting the importance of addressing cortical adaptations early after ACL injury and reconstruction. It remains unclear how improving corticomotor function would influence muscle strength and clinical outcomes; however, the results of this study warrant further investigation in this regard. 
Patients with knee osteoarthritis in the current study were older than all other ACL-R and healthy groups. However, age was not significantly correlated with any of the primary outcome measures, and therefore was not controlled for statistically in our analyses. Previous authors have identified age-related alterations in motor unit recruitment patterns. ${ }^{53}$ Knee extension strength is reportedly highest between the ages of 25-35, whereas a $15 \%$ decline may be expected per decade from 50-70 years. ${ }^{54}$ This may be in part due to the known reduction in motor neuron volume, leading to a spatial redistribution of motor unit fibers. ${ }^{55}$ The average age of patients with knee osteoarthritis was 45 years old, which ranged from 36-59. The involved osteoarthritic limb demonstrated more than a 38\% decline in MVIC torque compared to healthy controls, which appears to exceed the natural response to aging, although this relationship is not clear. Additionally, patients with knee osteoarthritis reported lower activity levels and perceived health status than healthy individuals and ACL-R patients, which may contribute to a decline of neuromuscular function.

\section{Implications}

Quadriceps weakness was observed among all patients with a history of ACL-R. It is unclear if early changes in strength, endurance, voluntary activation, and corticospinal excitability perpetuate long-term muscle dysfunction; however, the temporal relationships of these measures may be a contributing factor. While we cannot support this theory due to the cross-sectional design of this study, time from surgery was not correlated with any measure of quadriceps function listed above, suggesting that the underlying cause of persistent muscle dysfunction is multifactorial, and not related to time alone. If left unaddressed, the progressive nature of contributing factors may result in irreversible joint injury. Time is often used as a primary consideration when determining readiness for return to activity; ${ }^{56}$ however, previous authors have reported the recovery after ACL-R to be independent of time, which our results support. Therefore, it is possible that the progression of many factors other than time may be driving outcomes after ACL-R. 


\section{Limitations}

The natural history of neuromuscular outcomes over the long term following ACL reconstruction is unknown, and cannot be elucidated using a cross-sectional study design. To further understand the impact of ACL injury on the natural history of quadriceps neuromuscular function, longitudinal data in injured and uninjured cohorts are warranted. Future long-term studies may better address the natural course of muscle function following ACL reconstruction. Secondly, this study did not match groups by age. Age may influence outcomes after ACL-R; however, no measures of quadriceps function were correlated with age, supporting our decision to include these patients. In order to achieve a realistic case-series, it is reasonable to have age distributions among groups that are different. Future studies may consider matching by age to determine if the same relationships exist. Lastly, we were not able to verify the severity of knee osteoarthritis at the time of enrollment, which likely increased heterogeneity among ACL-R patients with and without known osteoarthritis. Likewise, we could not verify the absence of osteoarthritis in all other ACL-R patients. Radiographic changes are likely to precede the onset of clinical symptoms, which may have inflated the presence of osteoarthritis in patients beyond two years from ACL-R. Future investigations may consider assessment of neuromuscular function based on a clinical diagnosis in addition to radiographic evidence only.

\section{CONCLUSION}

Neuromuscular impairments are present in patients early and late after ACL reconstruction with and without knee osteoarthritis. Quadriceps strength and corticospinal excitability were impaired at all time points compared to healthy individuals, suggesting the role of addressing cortical function following ACL-R. Seemingly mal-adaptive strategies appear to have both peripheral and central origins. While time may play a role in the manifestation of specific neuromuscular adaptions following ACL-R, clinical outcomes are multifactorial and not likely influenced by time alone. 
Table $1_{i}$. Participant demographics and patient reported outcomes

\begin{tabular}{|c|c|c|c|c|}
\hline & $\begin{array}{l}\text { Healthy } \\
(\mathrm{N}=30)\end{array}$ & $\begin{array}{c}\text { Early ACL-R } \\
(\mathrm{N}=34)\end{array}$ & $\begin{array}{c}\text { Late ACL-R } \\
(\mathrm{N}=30)\end{array}$ & $\begin{array}{l}\text { Osteoarthritis } \\
(\mathrm{N}=8)\end{array}$ \\
\hline Age (years) & $22.7 \pm 4.6$ & $22.5 \pm 6.3 \S$ & $24.9 \pm 5.9 \S$ & $45.4 \pm 7.4 * \dagger \neq$ \\
\hline Mass $(\mathrm{kg})$ & $75.1 \pm 16.2$ & $73.9 \pm 16.9$ & $74.9 \pm 16.2$ & $85.2 \pm 24.8$ \\
\hline IKDC & $98.2 \pm 4.2$ & $81.5 \pm 13.4 * \S$ & $86.4 \pm 10.4 * \S$ & $62.9 \pm 15.2 * \dagger \ddagger$ \\
\hline KOOS Total $^{\mathrm{b}}$ & $98.7 \pm 2.5$ & $87.5 \pm 9.3^{*}$ & $92.1 \pm 6.0 * \S$ & $76.4 \pm 10.8^{*} \ddagger$ \\
\hline KOOS Activities of Daily Living ${ }^{b}$ & $99.8 \pm 0.7$ & $95.8 \pm 6.4^{*}$ & $97.8 \pm 3.1^{*}$ & $90.1 \pm 9.1^{*}$ \\
\hline KOOS Sport $^{\mathrm{b}}$ & $97.8 \pm 6.3$ & $76.5 \pm 21.1^{*}$ & $88.5 \pm 13.7 * \S$ & $53.8 \pm 29.4^{*} \ddagger$ \\
\hline KOOS Quality of Life & $96.5 \pm 9.8$ & $64.5 \pm 20.6^{*} \ddagger \S$ & $78.1 \pm 18.5^{*}+\S$ & $49.2 \pm 27.4^{*} \dagger \ddagger$ \\
\hline WOMAC Total $^{\mathrm{b}}$ & $0.3 \pm 0.8$ & $4.9 \pm 6.2^{*}$ & $3.2 \pm 2.9^{*}$ & $10.9 \pm 7.7^{*}$ \\
\hline WOMAC Pain ${ }^{b}$ & $19.8 \pm 0.6$ & $19.3 \pm 1.0 \S$ & $19.4 \pm 0.9 \S$ & $17.6 \pm 1.4^{*} \dagger \neq$ \\
\hline WOMAC Stiffness ${ }^{\text {b }}$ & $8.0 \pm 0.0$ & $7.0 \pm 1.4^{*}$ & $7.0 \pm 1.4^{*}$ & $6.5 \pm 1.5^{*}$ \\
\hline $\operatorname{VAS}(\mathrm{cm})^{\mathrm{b}}$ & $0.1 \pm 0.2$ & $0.7 \pm 0.9^{*} \ddagger$ & $0.2 \pm 0.5+\S$ & $1.2 \pm 0.8^{*} \ddagger$ \\
\hline Tampa Scale of Kinesiophobia & $28.6 \pm 5.8$ & $34.4 \pm 5.7^{*} \neq$ & $32.1 \pm 6.5+\S$ & $36.0 \pm 6.0^{*} \ddagger$ \\
\hline Veteran's Rand 12-Item Health & $86.0 \pm 7.6$ & $80.4 \pm 10.0 * \S$ & $82.4 \pm 6.7 \S$ & $68.9 \pm 14.2 * \dagger \ddagger$ \\
\hline Time since surgery (months) ${ }^{\mathrm{b}}$ & N/A & $9.0 \pm 4.3 \neq \S$ & $70.5 \pm 41.6+\S$ & $115.9 \pm 110.0 \dagger \neq$ \\
\hline \multicolumn{5}{|l|}{ Graft type, $\%$} \\
\hline Patellar tendon & N/A & $51.5 \%$ & $27.6 \%$ & $37.5 \%$ \\
\hline Hamstring & N/A & $42.4 \%$ & $48.3 \%$ & $62.5 \%$ \\
\hline Allograft & N/A & $6.1 \%$ & $24.1 \%$ & $0 \%$ \\
\hline Meniscectomy, \% & N/A & $35.5 \%$ & $37.0 \%$ & $75 \%$ \\
\hline Meniscus repair, \% & $\mathrm{N} / \mathrm{A}$ & $19.4 \%$ & $14.8 \%$ & $12.5 \%$ \\
\hline
\end{tabular}

Abbreviations: M, male; F, female; IKDC, International Knee Documentation Committee Subjective; KOOS, Knee Injury and

Osteoarthritis Outcome Score; WOMAC, Western Ontario and McMaster Universities Arthritis Index

${ }^{\mathrm{a}} \mathrm{Chi}$-squared analysis $\left(\chi^{2}\right)$

${ }^{\mathrm{b}}$ Non-normally distributed (Kruskal-Wallis Test)

* Different than healthy

t Different than early ACL-R

‡ Different than late ACL-R

$\S$ Different than ACL-R with knee osteoarthritis

Alpha level set at $p \leq .05$ 
Table $2_{\text {i. }}$. Quadriceps neuromuscular function in ACL-r patients and healthy individuals (means $\pm \mathrm{SD}$ )

\begin{tabular}{|c|c|c|c|c|c|c|c|}
\hline & & \multicolumn{3}{|c|}{ ACL-R } & \multicolumn{2}{|c|}{ Healthy } & \multirow[b]{2}{*}{$p$ value } \\
\hline & & Involved Limb & Uninvolved Limb & $p$ value $^{\mathrm{a}}$ & $\begin{array}{l}\text { Matched } \\
\text { Involved }\end{array}$ & $\begin{array}{c}\text { Matched } \\
\text { Uninvolved }\end{array}$ & \\
\hline \multirow[t]{3}{*}{ Knee Extension MVIC Torque (Nm/kg) } & Early & $1.9 \pm 0.6^{*}$ & $2.6 \pm 0.7$ & $<.001$ & $2.8 \pm 0.6$ & $2.7 \pm 0.6$ & .179 \\
\hline & Late & $2.2 \pm 0.6^{*}$ & $2.3 \pm 0.6^{*}$ & .029 & & & \\
\hline & $\mathrm{OA}$ & $1.7 \pm 0.7^{*}$ & $1.9 \pm 0.9 * \dagger$ & .331 & & & \\
\hline \multirow[t]{3}{*}{ Knee Extension Fatigue Index (\%) } & Early & $14.3 \pm 9.7 * \neq$ & $19.6 \pm 9.0^{*} \neq$ & .002 & $22.0 \pm 8.0$ & $22.2 \pm 8.2$ & .721 \\
\hline & Late & $21.7 \pm 7.6$ & $21.1 \pm 6.8$ & .557 & & & \\
\hline & $\mathrm{OA}$ & $21.6 \pm 6.8$ & $19.7 \pm 7.3$ & .299 & & & \\
\hline \multirow[t]{3}{*}{ Central Activation Ratio (\%) } & Early & $85.5 \pm 11.4^{*} \neq \S$ & $88.1 \pm 9.4 * \neq \S$ & .060 & $95.2 \pm 5.6$ & $93.8 \pm 6.6$ & .181 \\
\hline & Late & $90.5 \pm 8.4$ & $91.0 \pm 10.3$ & .696 & & & \\
\hline & $\mathrm{OA}$ & $92.6 \pm 6.8$ & $92.9 \pm 7.6$ & .902 & & & \\
\hline \multirow[t]{3}{*}{ Hoffmann Reflex (H:M ratio) } & Early & $0.19 \pm 0.19$ & $0.16 \pm 0.15$ & .147 & $0.14 \pm 0.12$ & $0.14 \pm 0.12$ & .988 \\
\hline & Late & $0.21 \pm 0.19$ & $0.19 \pm 0.16$ & .164 & & & \\
\hline & $\mathrm{OA}$ & $0.26 \pm 0.23$ & $0.30 \pm 0.32$ & .666 & & & \\
\hline \multirow[t]{3}{*}{ Active Motor Threshold (\% 2.0 Tesla) } & Early & $45.8 \pm 7.9 * \ddagger$ & $45.1 \pm 7.4^{*} \ddagger$ & .600 & $39.0 \pm 4.1$ & $39.0 \pm 3.4$ & .920 \\
\hline & Late & $42.8 \pm 9.1 * \S$ & $42.3 \pm 9.5 * \S$ & .601 & & & \\
\hline & $\mathrm{OA}$ & $50.8 \pm 7.6^{*} \ddagger$ & $48.3 \pm 7.1^{*} \ddagger$ & .106 & & & \\
\hline
\end{tabular}

Abbreviations: MVIC, maximal voluntary isometric contraction; OA, knee osteoarthritis

${ }^{a} p$ value for difference between limbs in ACL-R patients

${ }^{\mathrm{b}} p$ value for difference between limbs in healthy controls

* Different than healthy

† Different than early ACL-R

$\neq$ Different than late ACL-R

$\S$ Different than ACL-R with knee osteoarthritis

Alpha level set at $p \leq .05$ 

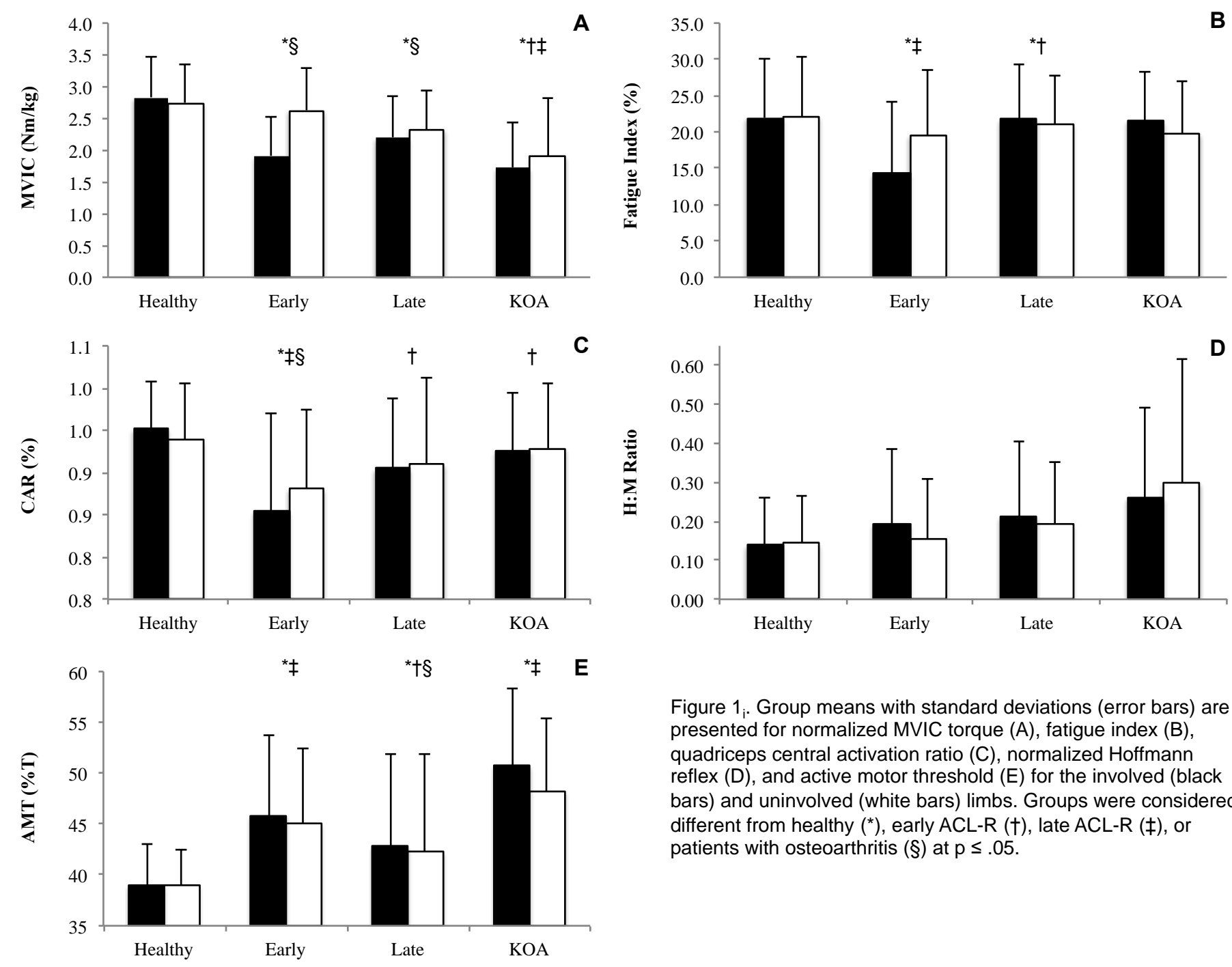

Figure 1. Group means with standard deviations (error bars) are presented for normalized MVIC torque (A), fatigue index (B),

quadriceps central activation ratio $(C)$, normalized Hoffmann reflex (D), and active motor threshold (E) for the involved (black bars) and uninvolved (white bars) limbs. Groups were considered different from healthy $\left({ }^{*}\right)$, early ACL-R $(\dagger)$, late ACL-R $(\ddagger)$, or patients with osteoarthritis $(\S)$ at $p \leq .05$. 


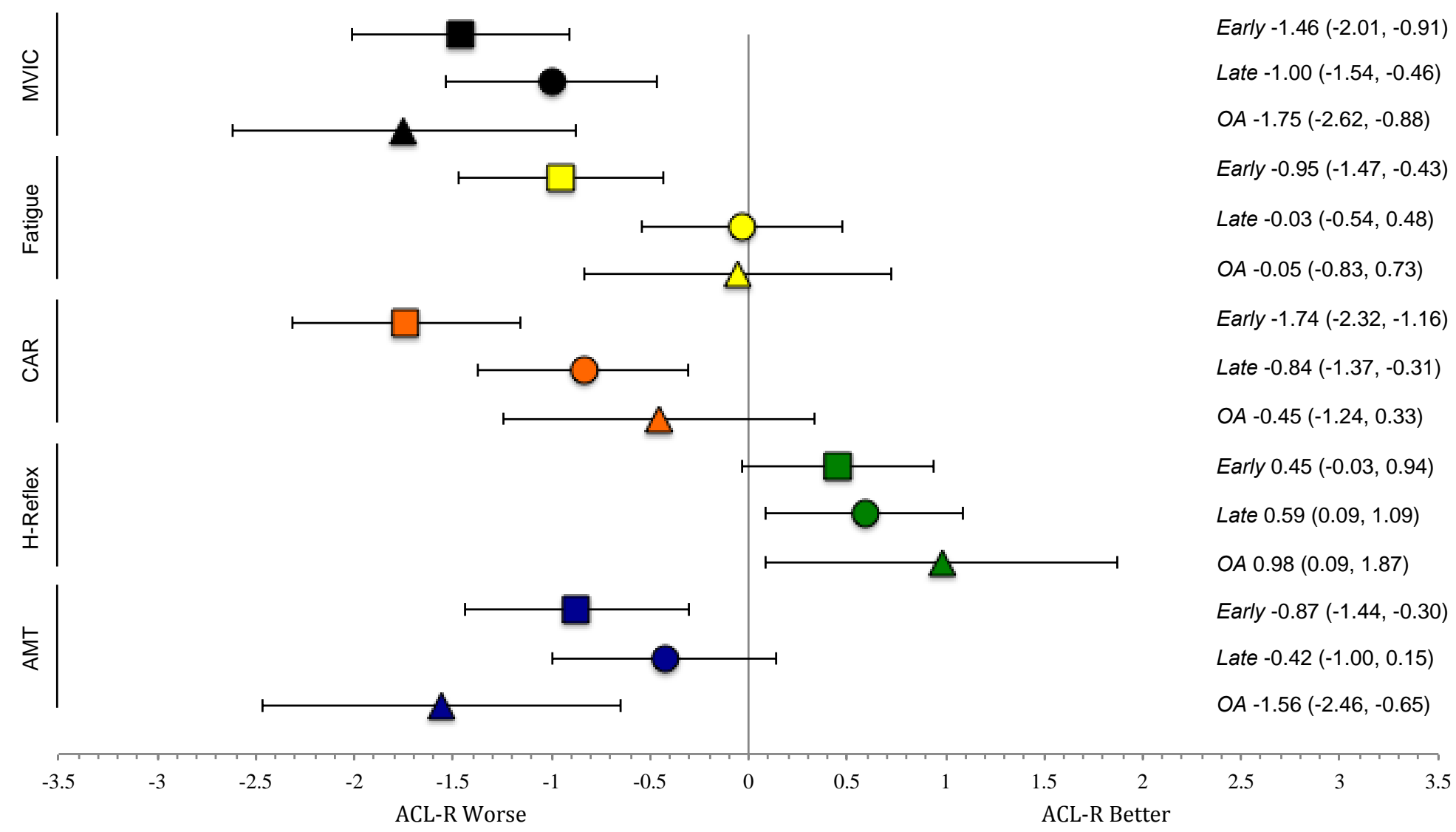

Figure $2_{i}$. Cohen's d effect sizes with $95 \%$ confidence intervals for involved limb MVIC torque, fatigue index, CAR, H-reflex, and AMT compared to matched health control. 


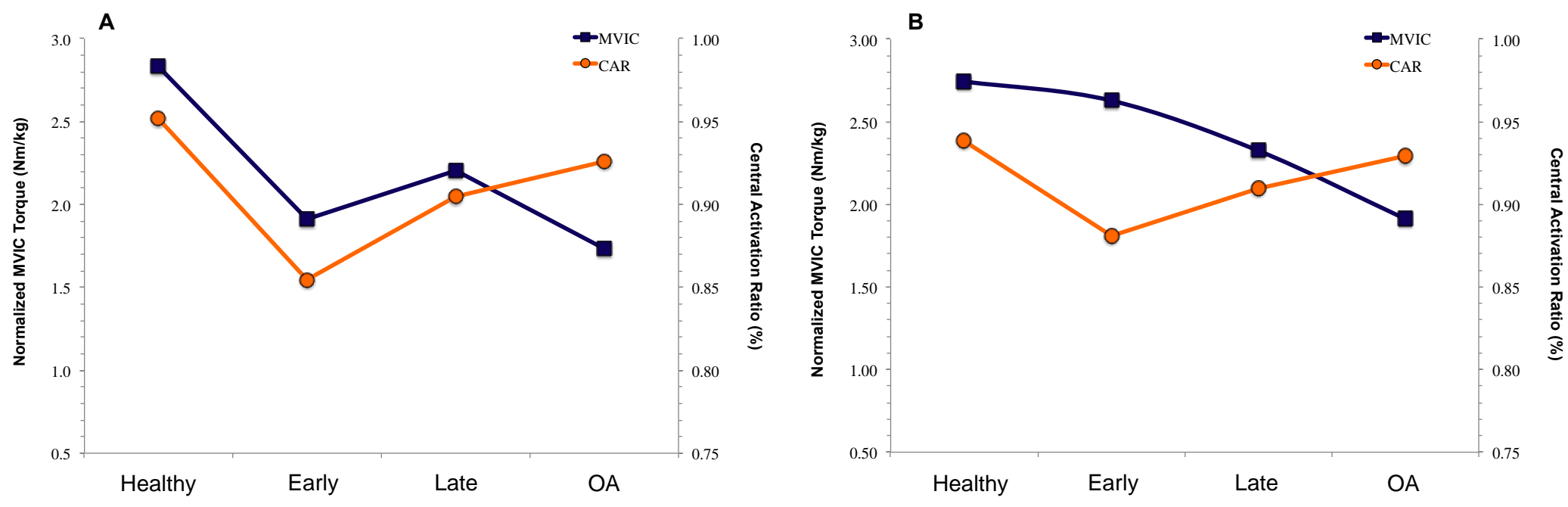

Figure $3_{\mathrm{A}}$. MVIC torque and quadriceps central activation ratio (CAR) of the involved (A) and uninvolved (B) limb in healthy, early ACL$\mathrm{R}$, late $\mathrm{ACL}-\mathrm{R}$, and $\mathrm{ACL}-\mathrm{R}$ with osteoarthritis (OA) patients. 
SECTION II: MANUSCRIPT II

RELATIONSHIP BETWEEN QUADRICEPS FUNCTION AND PATIENT REPORTED OUTCOMES IN ACL RECONSTRUCTED PATIENTS WITH AND WITHOUT KNEE OSTEOARTHRITIS 


\begin{abstract}
Context: The relationship between quadriceps muscle function and patient-reported outcomes over time after ACL reconstruction (ACL-R) is unclear. Understanding the relationships between muscle function and patient-reported outcomes may help clinicians better understand which factors may be affecting quality of life. Objective: To identify the relationship between objective measures of quadriceps function and patient-reported outcomes early $(<1$ year) and late $(>2$ years) after ACL-R, including patients who experienced post-traumatic knee osteoarthritis.

Design: Cross sectional. Setting: University laboratory. Patients or Participants: 72 ACL-R patients were categorized as early $(\mathrm{n}=34)$, late $(\mathrm{n}=30)$, or osteoarthritis $(\mathrm{n}=8)$.

Intervention(s): None. Main Outcome Measure(s): Isokinetic strength (peak torque, total work, average power), maximum voluntary isometric contraction (MVIC) torque, fatigue index, central activation ratio, spinal reflex excitability, and corticospinal excitability were measured bilaterally. The Knee Injury and Osteoarthritis Outcome Score (KOOS) and Veteran's Rand 12-Item Health Survey (VR-12) were used to quantify knee function and global health. Multiple linear (stepwise) regression analyses were used to predict patient reported outcomes in each group. Results: Early after ACL-R, knee extensor work, AMT symmetry, pain, and activity level explained $67.8 \%$ of variance in $\operatorname{KOOS}(p<.001)$; whereas, knee extensor work, activity level, and pain explained $53.0 \%$ variance in VR-12 $(p<.001)$. Late after ACL-R, age and isokinetic torque symmetry explained $28.9 \%$ of variance in $\operatorname{KOOS}(p=.004)$. In patients with osteoarthritis, kinesiophobia and isokinetic torque explained $77.8 \%$ of variance in $\operatorname{KOOS}$ score $(p=.010)$; whereas, activity level explained $86.4 \%$ variance in VR-12 $(p=.001)$. Conclusions: Factors of muscle function that are correlated with patient reported outcomes are different for patients early and late after ACL-R, and in those with knee osteoarthritis. These results support the importance of developing optimal evidence-based assessment strategies to identify impairments early after ACL-R.
\end{abstract}

Key Words: Isokinetic, limb symmetry, neuromuscular

Word Count: 300 


\section{INTRODUCTION}

Anterior cruciate ligament (ACL) injury and reconstruction (ACL-R) remains common among young and middle-aged, active individuals. ${ }^{1}$ ACL-R not only presents early challenges with regard to functional restoration, but also threatens long-term joint health, ${ }^{57}$ return to physical activity, ${ }^{58}$ and quality of life. ${ }^{59}$ Persistent quadriceps weakness is a particular interst to many

clinicians and researchers in this regard given its association with post-traumatic sequelae. ${ }^{7,10}$ The causal relationship between long-term quadriceps dysfunction (e.g. muscle weakness and decreased neuromuscular control) and knee joint degeneration has been proposed; ${ }^{7}$ however, the natural history of muscle function after ACL-R is not clear in this regard. Deleterious consequences of muscle weakness are commonly thought to develop over a long duration, yet significant joint space narrowing has been observed in patients within 4 years of reconstruction. ${ }^{11}$ In a recent investigation, quadriceps weakness has been identified at 20 -year follow up, ${ }^{60}$ supporting the temporal relationship between muscle function and joint health. As an alarming proportion of individuals may experience degenerative changes within the first and second decade from reconstruction, ${ }^{4}$ early identification of modifiable impairments are necessary.

Time from injury is a commonly reported criterion used in return to activity decisionmaking following ACL-R; ${ }^{56}$ however objective evidence-based measures of function are warranted. ${ }^{61}$ Recent authors ${ }^{62}$ have identified persistent physical impairments beyond the time of return to activity, recommending the inclusion of single-limb functional performance in addition to limb symmetry when making these decisions. While these data suggest that physical recovery from ACL-R may be independent of time, the relationship between physical impairments and perceived function may be in part mediated by time. Previous authors ${ }^{63}$ have established the diagnostic utility of both unilateral isometric knee extension torque and symmetric quadriceps central activation as strong indicators of self-reported knee function. ${ }^{63}$ Interestingly, symmetric quadriceps strength is reported to explain more variance $\left(\mathrm{R}^{2}=0.13\right.$ vs. 0.08$)$ in self-reported knee 
function than unilateral strength in patients 8.2 months post ACL-R. ${ }^{64}$ However, the relationship between unilateral function, limb symmetry, and time after ACL-R is not fully understood.

Establishing associations between disease and patient-oriented outcomes is a necessary step in developing evidence-based assessment paradigms. The association between quadriceps muscle function and patient-reported outcomes is widely studied; however, conflicting findings make it difficult for clinical recommendations. For example, isometric knee extension torque has been reported to explain between 7.8 and $61.0 \%$ of the variance in self-reported knee function at an average of $3.7^{65}$ and $4.5^{10}$ years post ACL-R respectively. These discrepancies may be in part due to the inclusion of patients at widespread time points after surgery. Risk for secondary or contralateral ACL injury is considerably greater during the two years following reconstruction, ${ }^{66}$ making this a clinically relevant time range. However, it is unclear how these relationships may change beyond two years post-op. Understanding the relationships between muscle function and patient-reported outcomes may help clinicians to better understand which factors may be affecting quality of life.

Therefore, the purpose of this study was to identify the relationship between objective measures of quadriceps function and patient-reported outcomes at early $(<1$ year $)$ and late $(>2$ years) durations after ACL-R, including patients who experienced post-traumatic knee osteoarthritis. We hypothesized that greater unilateral and more symmetric quadriceps function would be associated with improved patient-reported knee function and global health in patients early; whereas, symmetry would be most meaningful late, and unilateral function most meaningful in patients with knee osteoarthritis. Secondly, we aimed to identify which measures of quadriceps function best explained patient-reported function in each patient group, and hypothesized that strength would have the greatest influence on perceived outcomes.

\section{METHODS}

This was a cross-sectional study to investigate the influence of time from ACL-R on the relationship between quadriceps muscle function and patient-reported outcomes. Patients were 
compared at early (<1 year) and late ( $>2$ years) post-operative durations with and without knee osteoarthritis. Predictor variables for patient-reported function included isokinetic knee extension (peak torque, total work, average power) at $90 \% \mathrm{sec}$, knee extension MVIC torque, fatigue index (FI), central activation ratio (CAR), Hoffmann reflex (H-reflex), and active motor threshold (AMT). Dependent variables included regional knee function, measured by the Knee Injury and Osteoarthritis Outcomes Score (KOOS), and global health, measured by the Veteran's Rand 12Item Health Survey (VR-12).

\section{Participants}

72 patients with a history of primary, unilateral ACL-R participated in this study (table 1). Patients with a history of failed reconstruction, multiple ligament knee injury, treatable cartilage lesion, lower extremity joint surgery prior to ACL-R, lower extremity injury within 6 months other than ACL-R, concussion within 6 months, or neurological impairment were excluded from participation. Participants designated to the knee osteoarthritis group must have received a physician diagnosis after ACL-R based on radiographic evidence (Kellgren-Lawrence >1) in one or more compartment.

\section{Procedures}

Participants were asked to refrain from caffeine use and intense exercise within 12 hours of testing. ${ }^{16}$ Order of testing was maintained throughout the study, and counterbalanced by limb dominance (i.e. which limb would be used to kick a ball).

\section{Patient-Reported Function}

The $\operatorname{KOOS}^{25}$ has been established as a reliable and valid subjective assessment tool sensitive to detect change in knee function after ACL-R and osteoarthritis. The KOOS includes subdomains of pain, symptoms, activities of daily living, sport related function, and quality like. The total KOOS score was used to quantify 'knee function' in this study. Patient-reported outcomes following ACL-R commonly emphasize regional knee function. However, the impact of ACL injury on global health and quality of life are less represented. The VR-12 is an example 
of a 'global health' related quality of life measure that asks questions regarding general health, emotions, physical activity, pain, and personal feelings following injury. ${ }^{29}$ The VR-12 is similar to the Short Form-36, which has reported to be responsive to ACL-R, ${ }^{67}$ despite its widespread use in a variety of medical conditions. Activity level, pain, and fear of movement, were quantified using the Tegner Activity Scale, ${ }^{27}$ visual analog scale (VAS) for pain at rest, and the Tampa Scale of Kinesiophobia (TSK). ${ }^{28}$

\section{Spinal Reflex Excitability}

The H-reflex was used to quantify spinal reflex excitability as described. ${ }^{30,32}$ The area of greatest bulk over the vastus medialis was shaved, cleaned, and debrided. Two recording surface electromyography (EMG) electrodes were placed according to SENAM guidelines. ${ }^{31}$ A groundrecording electrode was placed over the contralateral distal anteromedial tibia. The EMG signal was digitally converted at $2000 \mathrm{~Hz}$ via 16-bit data acquisition system (MP150, BIOPAC Systems, Inc., Goleta, CA), band-pass filtered from 10 to $500 \mathrm{~Hz}$, and processed using Acqknowledge software (v. 4.2, BIOPAC Systems, Inc.). A stimulator module (STM100A, BIOPAC Systems, Inc.) and STMISOC current isolation unit were used to deliver an electrical stimulus to the femoral nerve. A dispersive carbon electrode was placed over the ipsilateral posterior thigh. A series of 1-millisecond square wave stimuli were sequentially administered until the maximum $\mathrm{H}$ reflex and muscle response (M-response) were identified. Three maximal H-reflexes were averaged and normalized to the average of three maximal M-responses (H:M ratio) for analysis.

\section{Isokinetic Strength}

Isokinetic knee extension peak torque, total work, and average power were assessed at $90^{\circ}$ per second using a Biodex multimodal dynamometer (System 3, Biodex Medical Systems, Inc., Shirley, NY). Participants were seated in $85^{\circ}$ hip flexion and $90^{\circ}$ knee flexion (anatomical reference) for the start of each test. A correction for limb weight was used. Participants were ensured a range of motion arc of $110^{\circ}$. Eight repetitions were completed at each testing speed with 45 seconds of rest between. An explanation of testing was provided, instructing participants 
to "kick out and pull back as hard and fast as possible." Participants were asked to keep their head and shoulders against the seat rest with arms crossed over their chest to minimize aberrant movement. ${ }^{68}$ Several repetitions were practiced at each speed to visualize proper technique. Participants were provided real time visual feedback via 50-inch LCD monitor to view progress during testing. Verbal encouragement was provided to ensure maximal effort. Isokinetic peak torque $(\mathrm{Nm} / \mathrm{kg})$, total work $(\mathrm{J} / \mathrm{kg})$, and average power $(\mathrm{W} / \mathrm{kg})$ were normalized to body mass.

\section{Isometric Strength and Quadriceps Central Activation}

Participants were seated in the multimodal dynamometer and completed a standardized acclimatization protocol, in which a series of submaximal trials $(25 \%, 50 \%, 75 \%$ perceived effort) were performed prior to recording three maximal effort trials with 60 seconds of rest between trials. A supramaximal percutaneous electrical stimulus was delivered to the quadriceps

using the superimposed technique ${ }^{33}$ during the third MVIC. Once the MVIC torque had reached a plateau consistent with previous trials, a square wave stimulator (S88, GRASS-TeleFactor, W. Warwick, RI) with isolation unit (SIU8T, GRASS-TeleFactor) was used to manually deliver a 100 -millisecond train of 10 square-wave pulses to the thigh via two self-adhesive electrodes ( 3 " $\mathrm{x}$ 5") placed over the proximal vastus lateralis and distal vastus medialis. Real time visual feedback was provided, and verbal encouragement given to ensure maximal effort during testing. Force data were digitally converted at $125 \mathrm{~Hz}$ via 16-bit data acquisition system, low-pass filtered at 10 $\mathrm{Hz}$, and processed using Acqknowledge software. The mean torque was calculated from a 100millisecond epoch during the maximal contraction plateau, or immediately prior to the SIB torque (MVIC 3 only). The MVIC torque epoch recorded from three maximal trials was averaged, and normalized to body mass $(\mathrm{Nm} / \mathrm{kg}$ ) to quantify quadriceps strength. Quadriceps CAR was calculated as previously described. ${ }^{34}$

\section{Quadriceps Fatigue Index}

Quadriceps FI was quantified during a 30-second knee extension MVIC task. ${ }^{35}$ Participants were instructed to "kick out as hard as possible and to maintain the contraction" 
while seated in the dynamometer in a similar fashion to quadriceps strength testing. A researcher prompted participants to start kicking, and the 30 -second trial began once the participant had achieved their perceived maximal effort. Verbal encouragement and visual feedback were omitted to minimize the occurrence of transient aberrant increases in torque. Force data were digitally converted at $125 \mathrm{~Hz}$ via 16-bit data acquisition system, low-pass filtered at $15 \mathrm{~Hz}$, and processed using Acqknowledge software. The mean torque was recorded during a series of 1-second epochs, and the greatest torque epoch from the first 5 seconds of the trial was recorded as the maximal torque $\left(\mathrm{T}_{\mathrm{Max}}\right)$. The area under the force-time curve (AUFC) for the entire contraction period for 0 to 30 seconds began at the time point of maximum muscle torque (TPM), and was used to quantify fatigue (Equation 1).

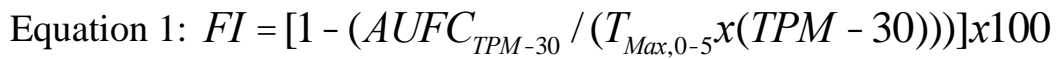

\section{Corticospinal Excitability}

The AMT was used to quantify corticospinal excitability via transcranial magnetic stimulation. Participants were again seated in the dynamometer similar to the aforementioned procedures. Surface EMG electrodes were replaced over the vastus medialis and distal anteromedial tibia for each limb. Participants wore a Lycra swim cap with bisecting lines and a 1 $\mathrm{cm} \times 1 \mathrm{~cm}$ grid to aid in the determination of the optimal stimulus location. Motor evoked potentials were elicited in the vastus medialis using a magnetic stimulator (MagStim Rapid, MagStim Company, Ltd., Wales, UK) with 110 mm double-cone coil. The AMT was determined by systematically reducing the stimulus intensity by $5 \%$ until a measurable MEP (>200 $\mu \mathrm{V})$

could no longer be elicited, then increased by $1 \%$ until its return for a minimum of 5 of 10 trials. $^{23}$ Real time visual feedback was used to aid participants during a force-matching task at $5 \%$ of the MVIC. EMG signals were digitally converted at $2000 \mathrm{~Hz}$ via 16-bit data acquisition system, band-pass filtered from 1 to $5000 \mathrm{~Hz}$, and processed using Acqknowledge software. 


\section{Limb Symmetry}

Unilateral data were assessed using the involved ACL-R limb, and limb symmetry indices (LSI) were calculated for each measure (Equation 2).

Equation 2: LSI = (Involved / uninvolved $) x 100$

\section{Statistical Analysis}

Group differences in demographics were assessed using separate one-way analyses of variance or Fisher's exact test. Pearson's product-moment correlations $(r)$ were used to identify the relationship between individual measures of quadriceps function and patient-reported function when normally distributed. Spearman's rank-order correlations $(\rho)$ were used in the event of nonnormally distributed data. Correlations were performed within each patient group (early ACL-R, late ACL-R, ACL-R with knee osteoarthritis) for KOOS and VR-12 separately. The absolute value of correlation coefficients was classified as very weak $(0.0-0.19)$, weak $(0.20-0.39)$, moderate $(0.40-0.59)$, strong $(0.60-0.79)$, or very strong $(0.80-1.0)$.

Separate multiple linear (stepwise) regression analyses were used to predict patient reported outcomes from measures of quadriceps function in each group. Only significantly correlated variables were considered for inclusion as predictors in our regression models. Variables were first assessed for multicollinearity, and those that were very strongly correlated with one another were reduced to only include the variable with the highest correlation. Probability thresholds for variable entry and removal were set at 0.05 and 0.10 respectively. Missing values were replaced with the mean for each respective group. The total $\mathrm{R}^{2}$, adjusted $\mathrm{R}^{2}$, and change in $\mathrm{R}^{2}$ were calculated for each step of the respective analysis. The level of statistical significance was set a priori at $P \leq .05$. All statistical analyses were performed using SPSS (version 20.0, IBM, Chicago, IL). 


\section{RESULTS}

Patient demographics are presented in table 1 . There were no differences in gender, height, or mass between groups $(p>.05)$. Patients with knee osteoarthritis were older than those without $(p<.05)$.

\section{Correlations}

Correlation coefficients are presented for each patient group in table 2. In patients early after ACL-R, KOOS scores exhibited strong correlations with isokinetic work, moderate correlations with isokinetic power and torque, isokinetic power and work symmetry, AMT symmetry, MVIC torque, and pain, and weak correlations with isokinetic torque symmetry, activity level, and kinesiophobia. VR-12 was moderately correlated with all measures of unilateral isokinetic strength and symmetry, MVIC torque, and activity level, and weakly correlated with pain and time since surgery.

In patients late after ACL-R, KOOS scores exhibited moderate correlations with isokinetic torque symmetry, and weak correlations with isokinetic work and power symmetry, and age. VR-12 was weakly correlated with fatigue index symmetry.

In patients with knee osteoarthritis after ACL-R, KOOS scores exhibited strong correlations with all measures of isokinetic strength, MVIC torque, and activity level. VR-12 was very strongly correlated with all measures of isokinetic strength, MVIC torque, and activity level. Multiple Regression

Regression results are presented for each group in tables 3-5. In patients early after ACL$\mathrm{R}$, normalized isokinetic work, active motor threshold symmetry, pain, and activity level predicted $67.8 \%$ of variance in $\operatorname{KOOS}$ score $\left(F_{4,29}=18.4, p<.001\right)$. Normalized isokinetic work, activity level, and pain predicted $53.0 \%$ variance in $\operatorname{VR}-12\left(F_{3,30}=13.4, p<.001\right)$.

In patients late after ACL-R, current age and peak torque symmetry predicted $28.9 \%$ of variance in KOOS score $\left(F_{2,27}=6.9, p=.004\right)$. There were no significant predictors for VR-12. 
In patients with knee osteoarthritis after ACL-R, kinesiophobia and normalized isokinetic torque predicted $77.8 \%$ of variance in $\operatorname{KOOS}$ score $\left(F_{2,5}=13.2, p=.010\right)$. Activity level predicted $86.4 \%$ variance in VR-12 $\left(F_{1,6}=37.9, p=.001\right)$ (Figure 1).

\section{DISCUSSION}

Measures of unilateral quadriceps muscle function and symmetry were correlated with patient-reported function early after ACL-R. In patients late after ACL-R, weak to moderate correlations were observed for only limb symmetry and patient-reported function. In contrast, unilateral measures of muscle function were strongly correlated with patient-reported function in patients with knee osteoarthritis. Knee extension isokinetic strength exhibited the strongest correlations with perceived knee function and global health in each patient group. The results of this study suggest that different factors predict patient reported outcomes at different time points after surgery, and that emphasis placed on a single outcome measure may not be the best strategy in evaluating all patients with ACL-R.

Our results indicate that both unilateral quadriceps function and symmetry are meaningfully associated with patient-reported function early after reconstruction. Improvements in unilateral quadriceps function and symmetry have been associated with improved knee function and lower extremity movement patterns at the time of return to sport. ${ }^{69,70}$ Quadriceps strength and performance symmetry $\geq 90 \%$ have been suggested as indicators of safe return to activity. ${ }^{71}$ For patients within a year after reconstruction, patient-reported outcomes may be best predicted by the combination of isokinetic knee extensor work in the involved limb, pain at rest, active motor threshold symmetry, and current activity level, suggesting that a single measure of muscle function may be insufficient to detect meaningful impairments related to perceived knee function and global health. Patients often experience a rapid decline in quadriceps strength and functional performance early after ACL-R, resulting in large asymmetries. ${ }^{72}$ This may be explained in part by early peripheral changes in muscle of the injured limb, ${ }^{50,51}$ as well as decreased central drive to muscle ${ }^{15}$ which may result in a functional decline of the contralateral 
limb. Bilateral responses to unilateral injury may confound estimates of limb symmetry, and appear to support the additional use of unilateral assessments to identify impairments early after ACL-R. This may explain why the combination of a unilateral measure of peripheral muscle function and bilateral measure of central nervous system function were able to predict knee function in patients early after ACL-R.

We observed a moderate negative correlation between knee function and active motor threshold symmetry in patients early after ACL-R. Unilateral quadriceps isometric strength and corticospinal excitability have been reported to explain $66 \%$ of the variance in self-reported knee function. ${ }^{10}$ Our results partially agree with these data in that measures of muscle function and patient demographics were able to explain $67.8 \%$ of self-reported knee function. Previous authors $^{22,73}$ have observed functional reorganization of the brain after ACL-R, with increased activation in attentional and sensory regions, suggestive of an increase in the cortical effort needed to complete a given task. In support of this, decreased corticospinal excitability, as measured by an increase in cortical motor thresholds, has been identified in patients as early as 6 months following ACL-R. ${ }^{15}$ Our results suggest that patients with lower bilateral cortical thresholds report improved knee function. In contrast to previous findings, isokinetic knee extensor work and active motor threshold symmetry alone accounted for $46.1 \%$ of the variation in knee function, which appear to highlight the importance of additional factors that may influence perceived function in patients early after ACL-R. While the relationship between quadriceps strength and self-reported function is commonly investigated, it is clear that muscle function alone does not dictate clinical outcomes. Based on the findings of this study, activity level, pain, fear of movement or re-injury, and time since surgery should be considered in the context of perceived outcomes within the first year from ACL-R

Weak to moderate correlations were observed between knee function and isokinetic knee extensor strength (peak torque, total work, and average power) symmetry in patients late after ACL-R. Interestingly, no other measure of muscle function was correlated in these patients, with 
the exception of fatigue index symmetry and global health; however, no variables were able to explain variance in global health. ACL injury and reconstruction is theorized to alter the natural history of muscle function; ${ }^{60,74}$ however, the trajectory of bilateral muscle function after unilateral injury is not clear. Inter-limb asymmetries are reported to be greater early after surgery, ${ }^{75}$ which may explain why measures of limb symmetry were correlated with patient-reported function early after ACL-R. Despite the observed relationships between isokinetic strength symmetry and knee function in patients late after ACL-R, peak torque symmetry was the only predictor of knee function, accounting for $12.5 \%$ of its variance. Compared to total work, which contributed $39.3 \%$ to the predictive ability in patients early after ACL-R, it appears that the relationship between unilateral muscle function and symmetry and patient reported outcomes is diminished beyond two years after ACL-R. It is possible that improved symmetry due to both improved ipsilateral muscle function and deterioration of the contralateral limb may mask persistent impairments. This may provide a false sense of good clinical outcomes and underestimate the presence of sub-clinical impairments, making assessment strategies difficult during this time frame. Beyond measures of muscle function, age was negatively correlated with knee function and accounted for $21.3 \%$ of its variance, indicating that younger patients reported improved knee function. Increased age at the time of surgery is a reported predictor of persistent muscle weakness up to 9 months after ACL$\mathrm{R} .^{76}$ Although age was able to explain nearly a quarter of the variance in knee function in the current study, the included measures of muscle function do not appear to have good predictive ability for perceived knee function or global health beyond two years in patients without osteoarthritis.

In patients with knee osteoarthritis after ACL-R, strong to very strong correlations between unilateral isokinetic and isometric quadriceps strength with knee function and global health were observed. The increased prevalence of osteoarthritis development in the contralateral limb has been observed at 20 years following unilateral ACL-R. ${ }^{57}$ This, in conjunction with the functional decline that may naturally occur over time in the contralateral limb, may help patients 
achieve symmetry despite having poor long-term outcomes. In support of this, our results suggest that unilateral muscle function is highly associated with knee function and global health in patients with knee osteoarthritis. Previous authors ${ }^{9,77}$ have reported strong correlations between unilateral quadriceps strength and self-reported function in patients with knee osteoarthritis. In further support of the importance of unilateral muscle function in patients with knee osteoarthritis, our results demonstrated that symmetry of muscle function was not correlated with patient-reported outcomes. Quadriceps weakness and activation failure is widely reported in patients with knee osteoarthritis, ${ }^{78}$ which may begin to explain why symmetry was not related to patient reported function in our study. Chronic bilateral deficits in voluntary quadriceps activation has been observed in patients with tibiofemoral osteoarthritis compared to healthy individuals. ${ }^{17}$ Therefore, symmetry values may be misleading in these patients since bilateral weakness is a possibility, which may explain the lack of observed correlations between patient-reported function and symmetry in the current study.

Beyond objective measure of muscle function, kinesiophobia was strongly correlated with knee function, and current activity level was very strongly correlated with global health in patients with knee osteoarthritis. These data indicate that individuals with less fear of movement were more likely to report higher perceived knee function, and those who were more physically active perceived better health status. The role of physical activity and muscle function is well established. Decreased physical activity, or inactivity, commonly occurs with ageing, and is reported to negatively influence muscle function. Patients with osteoarthritis in this study were 45 years old on average, yet reported lower activity levels than patients with no osteoarthritis, which may have influenced the relationship between physical impairments and perceived function. Interestingly, current activity level was the only predictor of global health in these patients, indicating that this may be an adequate surrogate for objective muscle function relative to perceived health status in patients with knee osteoarthritis. Both kinesiophobia and current activity level contributed to more than half of the variance explained in patient-reported 
outcomes, suggesting the need to look beyond traditional clinic or laboratory based measures of muscle strength in this patient population. By including a subset of patients with known osteoarthritis in this study, we were able better understand the long-term relationships between subjective and objective outcomes after ACL-R.

\section{Clinical Implications}

The results of this study suggest factors that are important during patient evaluations at different time points following ACL-R. The factors that are correlated with patient reported outcomes are different for patients early after ACL-R ( $<1$ year), late after ACL-R ( $>2$ years), and in patients with diagnosed knee osteoarthritis after ACL-R. Unilateral strength and symmetry are important factors early; however, as time progresses, symmetry may not be as useful to identify individuals with poor outcomes. Specifically, measures of isokinetic knee extensor strength appear to best predict patient-reported outcomes after ACL-R relative to other objective measures of muscle function. While very strong associations between unilateral measures of isokinetic and isometric quadriceps strength and patient-reported function were observed in patients with knee osteoarthritis, clinicians must be able to identify impairments early. The results of this study support the importance of developing optimal evidence-based assessment strategies to identify impairments early after ACL-R, and effectively guide patient care.

\section{Limitations}

The cross-sectional design of this study does not allow us to make conclusions based on the natural history of the relationship between quadriceps function, demographics, and patientreported function. Efforts were made to recruit a homogenous sample of patients after ACL-R; however, the purpose of the study was to investigate the relationships between subjective and objective outcomes over time, thus making it difficult to match groups on all demographics. We believe that the sample included in this study is representative of patients after ACL-R given the distribution of gender, graft type, and meniscal involvement. Additionally, we were not able to confirm the absence of osteoarthritis in patients early and late after ACL-R. 


\section{CONCLUSION}

Involved limb knee extensor work and corticospinal excitability symmetry explained patient outcomes early after ACL-R, suggesting the importance of assessing limb symmetry and unilateral function within the first year after surgery. Isokinetic knee extensor torque symmetry was only able to explain knee function to a lesser degree, making patient assessment beyond two years more challenging when using traditional measures of muscle function. Involved limb isokinetic knee extensor torque was only able to explain knee function in patients with osteoarthritis; however, outcomes were largely influenced by activity level and kinesiophobia in this group. These data support the inclusion of both objective measures of muscle function and patient-reported function when assessing patient outcomes, and suggest factors that are important during patient evaluations at different time points following ACL-R. Clinicians can use the information from this study to formulate an assessment that is specific to patients at different time points after surgery. 
Table $1_{\mathrm{ii}}$. Patient demographics (mean \pm standard deviation)

\begin{tabular}{|c|c|c|c|}
\hline & & Group & \\
\hline & Early $(n=34)$ & Late $(n=30)$ & Osteoarthritis $(n=8)$ \\
\hline Gender $^{a}$ & $20 \mathrm{M} / 14 \mathrm{~F}$ & $10 \mathrm{M} / 20 \mathrm{~F}$ & $2 \mathrm{M} / 6 \mathrm{~F}$ \\
\hline Age (years) & $22.5 \pm 6.3 \ddagger$ & $24.9 \pm 5.9 \ddagger$ & $45.4 \pm 7.4^{*} \dagger$ \\
\hline Height $(\mathrm{cm})$ & $174.1 \pm 11.0$ & $171.7 \pm 11.8$ & $170.0 \pm 9.7$ \\
\hline Mass (kg) & $73.9 \pm 16.9$ & $74.9 \pm 16.2$ & $85.2 \pm 24.8$ \\
\hline KOOS total & $87.5 \pm 9.3 † \ddagger$ & $92.1 \pm 6.0 * \ddagger$ & $76.4 \pm 10.8 * \dagger$ \\
\hline VR-12 & $80.4 \pm 10.0 \ddagger$ & $82.4 \pm 6.7 \neq$ & $68.9 \pm 14.2 * \dagger$ \\
\hline Tegner activity scale: current & $6.1 \pm 1.9 \ddagger$ & $6.9 \pm 1.6 \neq$ & $4.3 \pm 1.7 * \dagger$ \\
\hline Visual analog scale for pain $(\mathrm{cm})$ & $0.7 \pm 0.9 \dagger$ & $0.2 \pm 0.5^{*} \neq$ & $1.2 \pm 0.8^{*} \dagger$ \\
\hline Tampa Scale of Kinesiophobia & $34.4 \pm 5.7$ & $32.1 \pm 6.5$ & $36.0 \pm 6.0$ \\
\hline Time since surgery (months) & $9.0 \pm 4.3 \dagger \ddagger$ & $70.5 \pm 41.6^{*} \neq$ & $115.9 \pm 110.0 * \dagger$ \\
\hline \multicolumn{4}{|c|}{$\begin{array}{l}\text { Abbreviations: KOOS, Knee Injury and Osteoarthritis Outcome Score; VR-12, Veteran's Rand 12-Item Health } \\
\text { Survey } \\
\text { a Chi-squared analysis } \\
\text { * Different than early ACL-R } \\
\text { † Different than late ACL-R } \\
\text { † Different than ACL-R with osteoarthritis } \\
\text { Alpha level set at } p \leq .05\end{array}$} \\
\hline
\end{tabular}


Table $2_{\mathrm{ii}}$. Association between quadriceps function, patient demographics, and patient-reported function

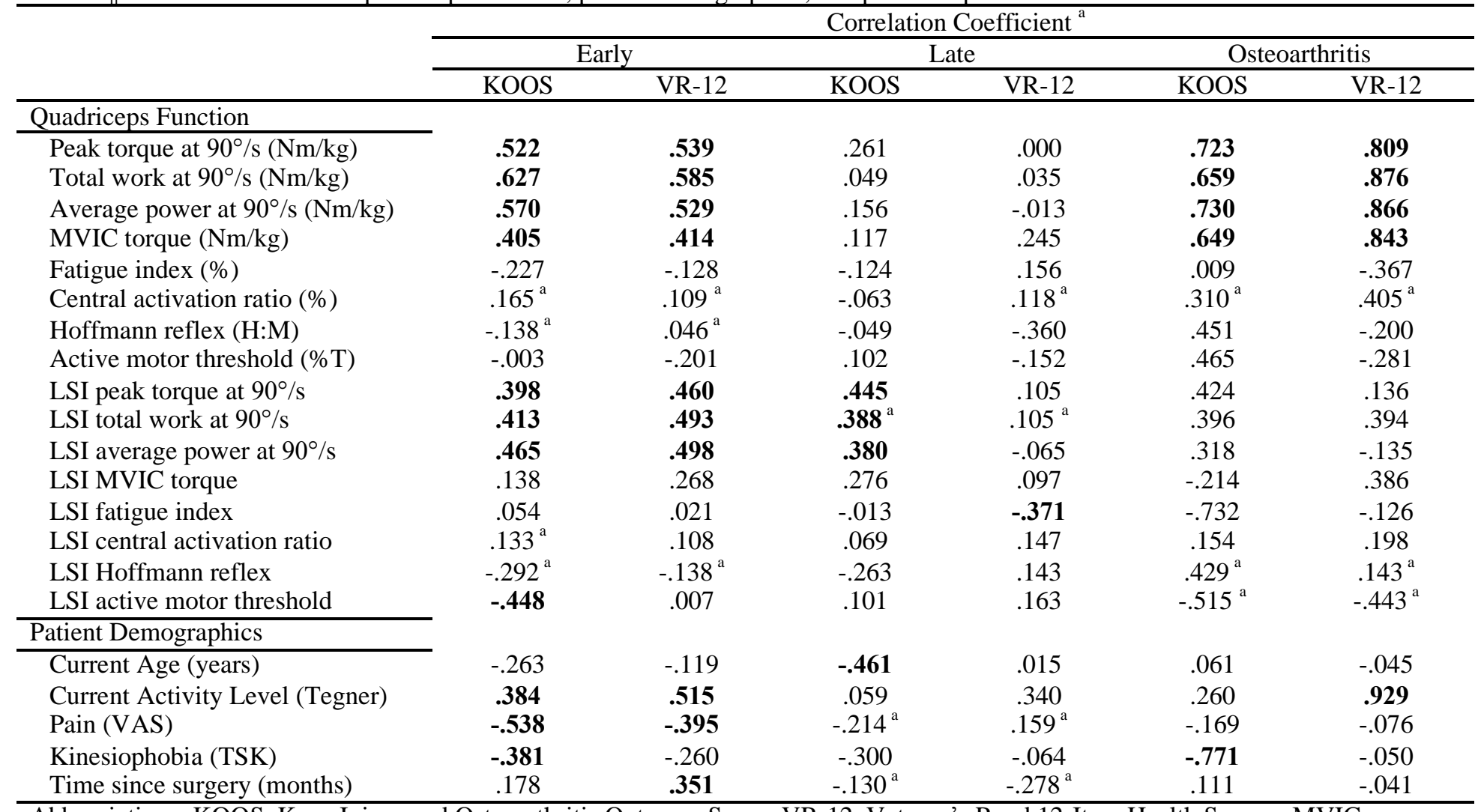

Abbreviations: KOOS, Knee Injury and Osteoarthritis Outcome Score; VR-12, Veteran's Rand 12-Item Health Survey; MVIC,

maximal voluntary isometric contraction; LSI, limb symmetry index; VAS, visual analog scale; TSK, Tampa Scale of Kinesiophobia

${ }^{\text {a }}$ Spearman's $\rho$

Bold indicates significant at $p \leq .05$ 
Table $3_{\mathrm{ii}}$. Final regression model in patients early after ACL-R

\begin{tabular}{|c|c|c|c|c|c|c|c|c|c|}
\hline \multirow[b]{2}{*}{ Step/Variable } & \multicolumn{4}{|c|}{ KOOS } & \multirow[b]{2}{*}{ Step/Variable } & \multicolumn{4}{|c|}{ VR-12 } \\
\hline & $\mathrm{R}^{2}$ & Adj. $R^{2}$ & $\Delta \mathrm{R}^{2}$ & $P$ value $^{\mathrm{a}}$ & & $\mathrm{R}^{2}$ & Adj. $R^{2}$ & $\Delta \mathrm{R}^{2}$ & $P$ value $^{\mathrm{a}}$ \\
\hline Total work & .393 & .374 & $.393^{*}$ & .001 & Total work & .342 & .322 & $.342^{*}$ & .006 \\
\hline Pain & .578 & .551 & $.185^{*}$ & $<.001$ & Activity level & .456 & .421 & $.114^{*}$ & .003 \\
\hline AMT Symmetry & .646 & .610 & $.068^{*}$ & .008 & Pain & .573 & .530 & $.116^{*}$ & .008 \\
\hline Activity level & .717 & .678 & $.071^{*}$ & .011 & & & & & \\
\hline
\end{tabular}

Abbreviations: KOOS, Knee Injury and Osteoarthritis Outcome Score; VR-12, Veteran's Rand 12-Item Health Survey; AMT, active motor threshold; Adj., adjusted $\mathrm{R}^{2} ; \Delta$, change in $\mathrm{R}^{2}$

${ }^{a} p$ value for individual variable in final model

${ }^{*} p \leq .05$

Table $4_{\mathrm{ij}}$. Final regression model in patients late after ACL-R

\begin{tabular}{|c|c|c|c|c|c|c|c|c|c|}
\hline \multirow[b]{2}{*}{ Step/Variable } & \multicolumn{4}{|c|}{ KOOS } & \multirow[b]{2}{*}{ Step/Variable } & \multicolumn{4}{|c|}{ VR-12 } \\
\hline & $\mathrm{R}^{2}$ & Adj. $\mathrm{R}^{2}$ & $\overline{\Delta R^{2}}$ & $P$ value $^{\mathrm{a}}$ & & $\mathrm{R}^{2}$ & Adj. $R^{2}$ & $\Delta \mathrm{R}^{2}$ & $P$ value $^{\mathrm{a}}$ \\
\hline Age & .213 & .185 & $.213^{*}$ & .024 & $\begin{array}{l}\text { No significant } \\
\text { predictors }\end{array}$ & - & - & - & - \\
\hline $\begin{array}{l}\text { Peak torque } \\
\text { symmetry }\end{array}$ & .338 & .289 & $.125^{*}$ & .032 & & & & & \\
\hline $\begin{array}{l}\text { Abbreviations: } \\
\text { change in } \mathrm{R}^{2} \\
{ }^{\mathrm{a}} p \text { value for in } \\
{ }^{*} p \leq .05\end{array}$ & $\begin{array}{l}\text { Knee } \\
\text { varia }\end{array}$ & $\begin{array}{l}\text { and } \mathrm{Os} \\
\text { inal } \mathrm{mo}\end{array}$ & & & & & & & \\
\hline
\end{tabular}

Table $5_{\mathrm{ij}}$. Final regression model in patients with knee osteoarthritis after ACL-R

\begin{tabular}{|c|c|c|c|c|c|c|c|c|c|}
\hline \multirow[b]{2}{*}{ Step/Variable } & \multicolumn{4}{|c|}{ KOOS } & \multirow[b]{2}{*}{ Step/Variable } & \multicolumn{4}{|c|}{ VR-12 } \\
\hline & $\mathrm{R}^{2}$ & Adj. $\mathrm{R}^{2}$ & $\Delta \mathrm{R}^{2}$ & $P$ value $^{\text {a }}$ & & $\mathrm{R}^{2}$ & Adj. $\mathrm{R}^{2}$ & $\Delta \mathrm{R}^{2}$ & $P$ value $^{a}$ \\
\hline Kinesiophobia & .595 & .527 & $.595^{*}$ & .025 & Activity level & .864 & .841 & $.864^{*}$ & .001 \\
\hline Peak torque & .841 & .778 & $.246^{*}$ & .039 & & & & & \\
\hline
\end{tabular}



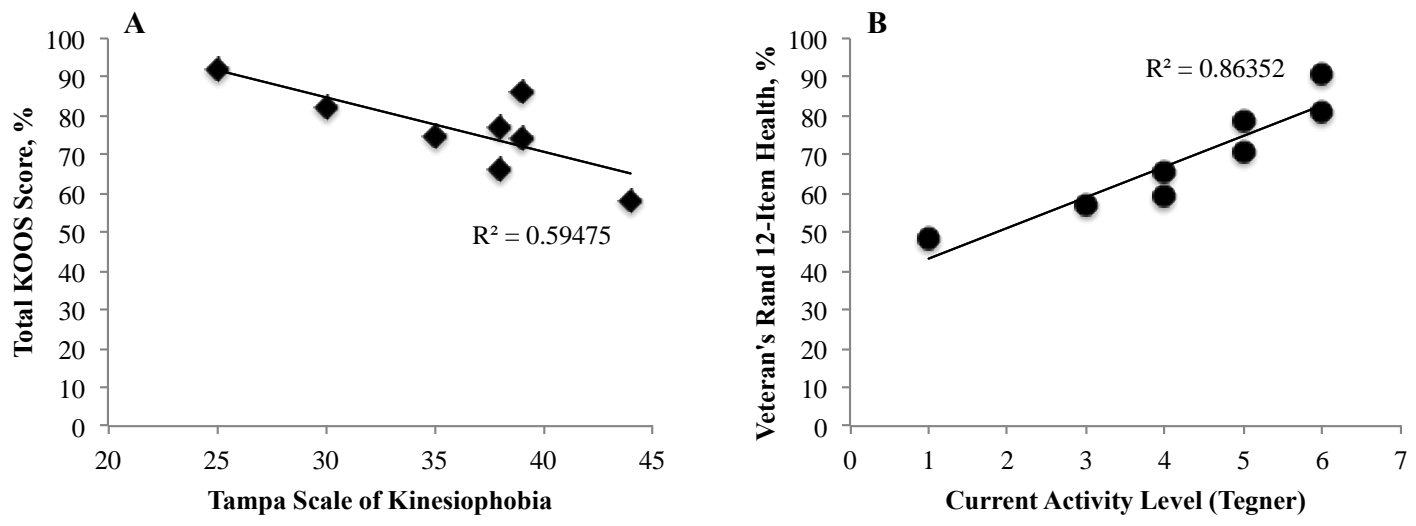

Figure $1_{\mathrm{ii}}$. Relationship between self-reported knee function and fear of movement (A) and between self-reported global health and current activity level (B). 


\section{SECTION II: MANUSCRIPT III}

QUADRICEPS AND PATIENT REPORTED FUNCTION IN ACL RECONSTRUCTED

PATIENTS: A PRINCIPAL COMPONENT ANALYSIS 


\begin{abstract}
Context: Assessment of physical function for individuals after ACL reconstruction (ACL-R) is complex and warrants the use of diverse evaluation strategies. By identifying tests that provide the most unique and meaningful information, a clearer understanding of post-traumatic muscle characteristics can be gained while maximizing the efficiency of assessment. Objective: To investigate the underlying constructs of quadriceps muscle function that uniquely describes aspects of performance in patients after ACL-R. Design: Cross sectional. Setting: University laboratory. Patients or Participants: 72 patients with a history of primary, unilateral ACL-R, and 30 healthy individuals participated. Intervention(s): None. Main Outcome Measure(s): Isokinetic strength (peak torque, total work, average power) at $90^{\circ}$ and $180^{\circ} /$ second, maximal voluntary isometric contraction (MVIC) torque, fatigue index, central activation ratio (CAR), Hoffmann reflex (H:M ratio), and active motor threshold (AMT) were measured bilaterally. Principal component analyses were performed for the involved limb, uninvolved limb, and limb symmetry. Receiver-operator-characteristic curve analyses were conducted to determine the diagnostic utility of each variable. Binary logistic regression was used to predict group membership (ACL-R vs. healthy). Results: Three components of peripheral, central, and combined muscle function were identified, which explained $70.7-80.5 \%$ of variance among measures of quadricep function. Total knee extensor work at $90 \% \mathrm{sec}(\geq 18.4 \mathrm{~J} . \mathrm{kg})$, AMT ( $\geq 39.5 \%$ ), and CAR ( $\geq 94.7 \%)$ of the involved limb were strong predictors of patient status, and correctly classified $83.5 \%$ of patients with ACL-R $(p<.001)$. Conclusions: Unique constructs of peripheral, central, and combined peripheral and central muscle function likely exist in ACL-R patients. Total knee extensor work at $90 \% \mathrm{sec}$, AMT, and quadriceps CAR consistently explained a significant portion of variance in measures of quadriceps function, demonstrated acceptable to excellent diagnostic utility, and predicted group membership with 72.8 to $83.5 \%$ accuracy.
\end{abstract}

Word Count: 282

Key Words: Active motor threshold, isokinetic torque, quadriceps activation 


\section{INTRODUCTION}

Clinical outcomes following ACL reconstruction (ACL-R) are often evaluated based on impairment and patient-reported function. The use of clinically meaningful tests is an important aspect of return to activity decision-making following ACL-R. Information from a variety of tasks including strength, performance, movement quality and perceived function have been recommended ${ }^{61,71}$ while evaluating athletes recovering from ACL-R. Unfortunately, the use of objective measures of function in clinical decision making remains sparse. A recent systematic review ${ }^{56}$ identified time since surgery as the most widely cited criterion used to determine readiness for return to activity after ACL-R, with subjective and/or objective criteria being far less utilized. The lack of consensus regarding the most appropriate criteria for releasing patients to activity following ACL-R may contribute to inconsistent reporting of standardized tests, or test batteries. $^{56,61}$

Assessment of physical function and health status for individuals with ACL-R is complex and warrants the use of diverse evaluation strategies. Multimodal assessments of peripheral muscle function (e.g. isokinetic and isometric quadriceps strength) are commonly used to evaluate outcomes after ACL-R. For example, persistent quadriceps weakness is well described, ${ }^{8,79}$ and remains a long-term concern in this population. Maximizing both unilateral strength and symmetry is reported to positively influence self-reported knee function after ACL$\mathrm{R} .{ }^{64}$ Quadriceps strength symmetry is commonly quantified, and is believed to be an important factor in landing mechanics, ${ }^{69,80}$ functional outcomes, ${ }^{70,81}$ and perceived function. ${ }^{63}$ However, bilateral alterations in quadriceps strength are believed to arise from a centrally mediated response to unilateral peripheral joint injury, ${ }^{14,18,82}$ which may confound measures of limb symmetry. For example, bilateral quadriceps activation failure has been identified in patients after ACL-R, ${ }^{8}$ suggesting the need to include measures of centrally-mediated muscle function (e.g. voluntary activation, spinal reflex excitability, corticospinal excitability) in assessment batteries. The wealth of data available to clinicians may be overwhelming since there are many possible 
measures that may help guide treatment decision-making. By identifying tests that provide the most useful information, clinicians and researchers can utilize a consistent set of measures, and improve the ability to assess patient outcomes with the fewest and most important tests.

Some potential factors in performing large test batteries are the time requirement from clinician and patient, expensive and technically demanding equipment, and the concern for exposing patients to unnecessary risk with extensive testing. In an effort to minimize risk, and maximize the efficiency of an assessment program, we aim to identify tests that provide the most meaningful information about a population of interest. Principal component analysis (PCA) is an analytical technique that can help in this regard by reducing the dimensionality of a larger set of measures to provide a clearer interpretation of underlying constructs that best characterize a given population. By further establishing the diagnostic and predictive abilities of assessment tools to discriminate between patients with and without ACL-R, clinicians and researchers can begin to evaluate the utility of each, and work towards an evidence-based assessment.

Therefore, the purpose of this study was to investigate the underlying constructs of lower extremity muscle function that uniquely describe aspects of performance in ACL-R individuals. We hypothesized that clinically relevant clusters of data would be identified and that each would provide unique and meaningful information. A secondary purpose was to establish clinical thresholds for measures of quadriceps function to classify patients with and without ACL-R.

\section{METHODS}

This was a descriptive laboratory study to investigate quadriceps neuromuscular and patient-reported function among ACL-R individuals and healthy controls. Independent variables included group (ACL-R, healthy) and limb (involved, uninvolved). Dependent variables included isokinetic strength (peak torque, total work, average power) at $90^{\circ}$ and $180^{\circ}$ per second, maximal voluntary isometric contraction (MVIC) torque, fatigue index, central activation ratio (CAR), Hoffmann reflex (H-reflex), and active motor threshold (AMT). The International Knee Documentation Committee (IKDC) subjective knee evaluation form, ${ }^{24}$ Knee Injury and 
Osteoarthritis Outcome Score (KOOS), ${ }^{25}$ and Western Ontario and McMaster Universities Osteoarthritis Index (WOMAC) ${ }^{26}$ were used to quantify regional knee function. The Tampa Scale of Kinesiophobia (TSK) ${ }^{28}$ and Veteran's Rand 12-Item Health Survey (VR-12) ${ }^{29}$ were used to quantify psychological function. The Tegner Activity Scale ${ }^{27}$ and Godin Leisure-Time Exercise questionnaire ${ }^{83}$ were used to quantify activity related function.

\section{Participants}

A total of 102 individuals volunteered for this study, including 72 patients with a history of primary, unilateral ACL-R and 30 healthy individuals between the ages of 15 and 65 (table 1). Patients were excluded if they had a lower extremity joint surgery prior to ACL-R, multiple ligament reconstruction, history of graft failure, contralateral knee surgery, lower extremity injury within 6 months, concussion within 6 months, neurological disorder, or were currently taking a centrally acting medication ${ }^{23}$ at the time of enrollment. Graft type and meniscal involvement were reported descriptively, but not used to determine eligibility. Healthy participants were excluded if they reported a lower extremity joint injury requiring care within 6 months or history of concussion. Our University's Institutional Review Board for Health Sciences Research approved this study, and all participants provided informed consent prior to enrollment.

\section{Procedures}

All testing was performed bilaterally during a single study visit. Participants were asked to refrain from caffeine use and intense exercise within 12 hours prior to participation. ${ }^{16}$ Testing always occurred in the following sequence: H-reflex, isokinetic strength, MVIC torque, CAR, fatigue index, and AMT. Limb dominance was determined by asking participants which limb would be used to kick a ball. The order of testing was counterbalanced by limb dominance to account for a potential learning effect.

\section{Spinal Reflex Excitability}

The H-reflex was used as previously described ${ }^{30}$ to quantify spinal-mediated inhibition of the quadriceps. The area of greatest bulk over the vastus medialis was shaved, cleaned, and 
debrided. Two recording surface electromyography (EMG) electrodes were placed according to SENAM guidelines. ${ }^{31}$ A ground-recording electrode was placed over the contralateral distal anteromedial tibia. The EMG signal was digitally converted at $1000 \mathrm{~Hz}$ via 16-bit data acquisition system (MP150, BIOPAC Systems, Inc., Goleta, CA), band-pass filtered from 10 to $500 \mathrm{~Hz}$, and processed using Acqknowledge software (v. 4.2, BIOPAC Systems, Inc.). A stimulator module (STM100A, BIOPAC Systems, Inc.) and STMISOC current isolation unit were used to deliver an electrical stimulus to the femoral nerve. A dispersive carbon electrode was placed over the ipsilateral posterior thigh. A series of 1-millisecond stimuli were sequentially administered until the maximum H-reflex and muscle response (M-response) were identified. The average of three maximal responses was recorded for each measure, and the H-reflex was normalized the M-response (H:M ratio) for final analysis. Strong within-session reliability $\left(\mathrm{ICC}_{3, \mathrm{k}}\right.$ $=0.987$ ) has been reported when using the average of three measures. ${ }^{30}$

\section{Isokinetic Strength}

Isokinetic peak torque, total work, and average power were assessed at $90^{\circ}$ and $180^{\circ}$ per second using a Biodex multimodal dynamometer (System 3, Biodex Medical Systems, Inc., Shirley, NY). Participants were seated in $85^{\circ}$ hip flexion and $90^{\circ}$ knee flexion (anatomical reference) for the start of each test. A correction for limb weight was used. Participants were ensured a range of motion arc of $110^{\circ}$. Eight repetitions were completed at each testing speed with 45 seconds of rest between. An explanation of testing was provided, instructing participants to "kick out and pull back as hard and fast as possible." Participants were asked to keep their head and shoulders against the seat rest with arms crossed over their chest to minimize aberrant movement. ${ }^{68}$ Several repetitions were practiced at each speed to visualize proper technique. Participants were provided real time visual feedback via 50-inch LCD monitor to view progress during testing. Verbal encouragement was provided to ensure maximal effort. Data were normalized to body mass for peak torque $(\mathrm{Nm} / \mathrm{kg})$, total work $(\mathrm{J} / \mathrm{kg})$, and average power $(\mathrm{W} / \mathrm{kg})$. 


\section{Knee Extension MVIC Torque and Voluntary Activation}

Participants were seated in the multimodal dynamometer and completed a standardized acclimatization protocol, in which a series of submaximal trials $(25 \%, 50 \%, 75 \%$ perceived effort) were performed prior to recording three maximal effort trials with 60 seconds of rest between trials. A supramaximal percutaneous electrical stimulus was delivered to the quadriceps using the superimposed technique ${ }^{33}$ during the third MVIC. Once the MVIC torque had reached a plateau consistent with previous trials, a square wave stimulator (S88, GRASS-TeleFactor, W. Warwick, RI) with isolation unit (SIU8T, GRASS-TeleFactor) was used to manually deliver a 100 -millisecond train of 10 square-wave pulses to the thigh via two self-adhesive electrodes (3" $\mathrm{x}$ 5") placed over the proximal vastus lateralis and distal vastus medialis. Real time visual feedback was provided, and verbal encouragement given to ensure maximal effort during testing. Force data were digitally converted at $125 \mathrm{~Hz}$ via 16-bit data acquisition system, low-pass filtered at 10 $\mathrm{Hz}$, and processed using Acqknowledge software. A 100-millisecond epoch was recorded during a stable region from MVIC 1 and 2, and immediately prior to the resultant superimposed burst torque $\left(\mathrm{T}_{\mathrm{SIB}}\right)$ of MVIC 3. MVIC torque $\left(\mathrm{T}_{\mathrm{MVIC}}\right)$ were normalized the body mass $(\mathrm{Nm} / \mathrm{kg})$. The central activation ratio (CAR) was used to quantify voluntary activation (Equation 1). ${ }^{34}$

Equation 1: $C A R=T_{M V I C} /\left(T_{M V I C}+T_{S I B}\right)$

\section{Fatigue Index}

Quadriceps fatigue was quantified during a 30-second knee extension MVIC task. ${ }^{35}$ Participants were instructed to "kick out as hard as possible and to maintain the contraction" while seated in the dynamometer in a similar fashion to quadriceps strength testing. Participants were prompted to start kicking, and the 30-second trial began once the participant had achieved their perceived maximal effort. Verbal encouragement and visual feedback were omitted to minimize the occurrence of transient aberrant increases in torque. Force data were digitally converted at $125 \mathrm{~Hz}$ via 16-bit data acquisition system, low-pass filtered at $15 \mathrm{~Hz}$, and processed 
using Acqknowledge software. The mean torque was recorded during a series of 1-second epochs, and the greatest torque epoch from the first 5 seconds of the trial was recorded as the maximal torque $\left(\mathrm{T}_{\mathrm{Max}}\right)$. The fatigue index was calculated as the area under the force-time curve (AUFC) for the entire contraction period for 0 to 30 seconds, which began at the time point of maximum muscle torque (TPM) (Equation 2).

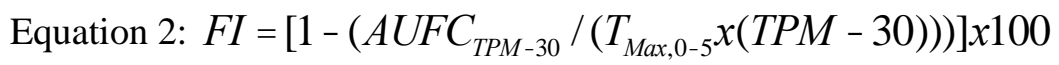

\section{Corticospinal Excitability}

Active motor threshold (AMT) was used to quantify corticospinal excitability via transcranial magnetic stimulation. Participants were again seated in the dynamometer similar to the aforementioned procedures. Surface EMG electrodes were replaced over the vastus medialis and distal anteromedial tibia for each limb. Participants wore a Lycra swim cap with bisecting lines and a $1 \mathrm{~cm} \times 1 \mathrm{~cm}$ grid to aid in the determination of the optimal stimulus location. Motor evoked potentials were elicited using a magnetic stimulator (MagStim Rapid, MagStim Company, Ltd., Wales, UK) with 110 mm double-cone coil. The AMT was determined by systematically reducing the stimulus intensity by $5 \%$ until a measurable MEP $(>200 \mu \mathrm{V})$ could no longer be elicited, then increased by $1 \%$ until its return for a minimum of 5 of 10 trials. ${ }^{23,84}$ Real time visual feedback was used to aid participants during a force-matching task at $5 \%$ of the MVIC. EMG signals were digitally converted at $2000 \mathrm{~Hz}$ via 16-bit data acquisition system, band-pass filtered from 1 to $5000 \mathrm{~Hz}$, and processed using Acqknowledge software.

\section{Limb Symmetry}

Limb symmetry indices (LSI) were calculated for each dependent variable of quadriceps function in the ACL-R group (Equation 3) and healthy control group (Equation 4). The ACL-R limb was identified as the dominant limb in $57 \%$ of ACL-R patients. Therefore, a random number generator was used to randomly assign $57 \%$ of the healthy control dominant limbs as the "matched ACL-R limb” for analysis. 
Equation 3: $L S I_{A C L R}=A C L \quad R \lim b /$ contralteral $\lim b$

Equation 4: $L S I_{C O N}=$ MatchedACL $R \lim b /$ matchedcontralteral $\lim b$

\section{Statistical Analysis}

Criteria to determine the appropriate sample size when using PCA is variable, with recommended subject-to-variable ratios ranging 3:1 to 20:1. In the current study, PCA was performed on a sample of 72 ACL-R patients using 11 variables per model, which resulted in a 6.5:1 subject-to-variable ratio. Although no absolute threshold for minimum sample size exists, 50 samples are generally considered a minimum. The Kaiser-Meyer-Olkin (KMO) measure was used to verify that our sample was adequate, where a value greater than 0.5 indicates adequate sample size.

All normally distributed data were compared between groups using independent $t$-tests, and non-normally distributed data were compared using Mann-Whitney U tests. Gender was compared between groups using a Chi-squared test.

PCA was performed via principal component extraction on ACL-R patients only. Separate analyses were conducted for quadriceps function and patient-reported function. Data were analyzed with and without varimax rotations in an attempt to uncover simple structure. Both theoretical and empirical evidence were considered when deciding on the number of components to retain in the final model. Separate analyses were conducted using data from the (1) involved limb, (2) uninvolved limb, and (3) limb symmetry indices. Missing values were replaced with the grand mean from ACL-R patients. The decision to retain a component was made if the unrotated component exceeded an eigenvalue of 1.0, met Horn's parallel analysis and minimum scree requirement, and explained an appreciable percentage of total variance ( $\geq 5 \%)$. Individual variables within each component were initially retained if they explained a minimum of $10 \%$ of the variance (loaded $\geq 0.3$ ). Bivariate Pearson product-moment correlations $(r)$ were conducted among variables that met these criteria to determine the presence of multicollinearity $(r \geq 0.9)$ or 
lack of association $(r<0.3)$ within each component. Variables were retained based on strength of loading, redundancy in variance explained, the ability to distinguish between healthy and ACL reconstructed patients (based on $p$ value and Cohen's $d$ effect size), and diagnostic utility.

Receiver operator characteristic (ROC) curve analyses were conducted to determine the diagnostic utility of each variable. The area under the ROC curve (AUC) represents the ability of a diagnostic tool to correctly assign a patient to an appropriate diagnostic category, and was used to evaluate each variable, where an AUC of $0.5=$ no discrimination, $0.7-0.8=$ acceptable discrimination, $0.8-0.9=$ excellent discrimination, and $>0.9=$ outstanding discrimination. Cutoff values that maximized both sensitivity and specificity were visually selected as thresholds to classify group membership (ACL-R vs. healthy) for all retained variables.

Binary logistic regression was used to predict group membership (ACL-R vs. healthy) using the combination of variables with the greatest discriminatory value in each retained component. Included variables were entered into a regression model for the involved limb, uninvolved limb, and limb symmetry. All variables entered into each model were retained to determine the predictive ability of each test battery. Missing values were replaced with the grand mean for ACL-R patients. The accuracy of classification, sensitivity, specificity, positive likelihood ratio, and negative likelihood ratio was calculated for each model. The level of statistical significance was set a priori at $p \leq .05$. All statistical analyses were performed using SPSS (version 20.0, IBM, Chicago, IL).

\section{RESULTS}

\section{Between Group Comparisons}

Demographics and patient-reported function are presented in table 1. Groups did not significantly differ by gender, age, height, or mass $(p>.05)$. Means and standard deviations for measures of quadriceps function are presented in table 2. 


\section{Principal Component Analysis}

PCA for the involved limb revealed a three-component solution that explained $80.5 \%$ of the cumulative variance (table 3). Component one accounted for $59.1 \%$ variance, and included all measures of isokinetic strength and knee extension MVIC torque, which were strongly correlated with one another $(r>0.7)$. Component two accounted for $10.7 \%$ variance, and included the H:M ratio, and AMT, which were weakly correlated $(r=-.168, p=.177)$. Component three accounted for $10.7 \%$ variance, and included CAR and fatigue index, which were weakly correlated $(r=$ $.083, p=.486)$.

PCA for the uninvolved limb revealed a two-component solution that explained $70.7 \%$ of the cumulative variance (table 4). Component one accounted for $56.1 \%$ variance, and included all measures of isokinetic torque and knee extension MVIC torque, which were strongly correlated with one another $(r>0.7)$. Component two accounted for $14.6 \%$ variance and included AMT, $\mathrm{H}: \mathrm{M}$ ratio, CAR, and fatigue index, which were weakly correlated with one another $(r<0.3)$.

PCA for limb symmetry revealed a three-component solution that explained $78.9 \%$ of the cumulative variance (table 5). Component one accounted for 54.0\% variance, and included all measures of isokinetic strength and knee extension MVIC torque, which were strongly correlated with one another $(r>0.7)$. Component two accounted for $14.9 \%$ variance, and included fatigue index and CAR, which were moderately correlated $(r=-.311, p=.008)$. Factor three accounted for $10.0 \%$ variance, and included AMT and H:M ratio, which were weakly correlated $(r=.041, p$ $=.751)$.

PCA for patient reported function revealed a two-component solution that explained $70.7 \%$ of the cumulative variance among variables (table 6). Component one accounted for 43.7\%, and included IKDC, KOOS, WOMAC, and TSK. Component two accounted for $27.0 \%$ variance, and included the Godin, Tegner, and VR-12. 


\section{Receiver Operator Characteristic Curve (Discriminatory Value)}

Involved limb total work at $90^{\circ} / \mathrm{sec}, \mathrm{AMT}$, and CAR yielded the highest discriminatory ability for each respective factor (table 3). Cut-off values of $18.4 \mathrm{~J} / \mathrm{kg}$ (sensitivity: 0.83 , specificity: 0.77 ), 39.5\% (sensitivity: 0.77 , specificity: 0.62), and 94.7\% (sensitivity: 0.71 , specificity: 0.69) were established to discriminate between groups for each respective variable.

Uninvolved limb total work at $90 \% \mathrm{sec}$ and AMT yielded the highest discriminatory ability for each respective factor (table 4). Cut-off values of $18.9 \mathrm{~J} / \mathrm{kg}$ (sensitivity: 0.61, specificity: 0.80 ) and $41.5 \%$ (sensitivity: 0.68 , specificity: 0.78 ) were established to discriminate between groups for each respective variable.

Limb symmetry indices for peak torque at $180^{\circ} / \mathrm{sec}, \mathrm{CAR}$, and AMT had the highest discriminatory ability for each respective factor. Cut-off values of 0.93 (sensitivity: 0.78 , specificity: 0.83), 1.01 (sensitivity: 0.72, specificity: 0.48 ), and 1.02 (sensitivity: 0.67, specificity: 0.53 ) were established to discriminate between groups for each respective variable.

\section{Logistic Regression (Predictive Value)}

Regression results are presented in table 7 . Involved limb, total work at $90 \% \mathrm{sec}$, AMT, and CAR explained $49.0 \%$ (Nagelkerke $\mathrm{R}^{2}$ ) of the variance in the presence of ACL-R, and correctly classified $83.5 \%$ of ACL-R cases; $\chi^{2}(3)=35.6, p<.001$.

Uninvolved limb total work at $90 \%$ sec and AMT explained $22.0 \%$ (Nagelkerke $\mathrm{R}^{2}$ ) of the variance in the presence of ACL-R, and correctly classified $72.8 \%$ of ACL-R cases; $\chi^{2}(2)=14.8$, $p=.001$

Limb symmetry indices of peak torque at $180 \% \mathrm{sec}$, CAR, and AMT explained $30.9 \%$ (Nagelkerke $\mathrm{R}^{2}$ ) of the variance in the presence of ACL-R, and correctly classified $79.5 \%$ of ACL-R cases; $\chi^{2}(3)=19.6, p<.001$.

\section{DISCUSSION}

The results of this study support our hypothesis that individual constructs of peripheral and central nervous system function describe unique aspects of lower extremity function and 
performance in ACL-R patients. Based on discriminatory and predictive ability, we propose a rationale for a reduced test battery that can be considered when making decisions about return to activity in ACL-R individuals. Identifying objective and clinically useful test batteries are essential to develop evidence-based assessments. By taking into account the ability to differentiate between individuals with and without pathology using a multimodal approach, clinicians and researchers can begin to advance patient care.

\section{Patient-Reported Outcomes and Quadriceps Function}

Identifying individual subjective and objective tests that differentiate individuals with and without ACL-R may be helpful to determine which are most important to optimize patient assessment. In the current study, ACL-R patients demonstrated a reduction in all measures of patient-reported function compared to healthy controls. Patients with higher self-reported knee function may be more likely to present with higher unilateral and more symmetric quadriceps strength, with individuals reporting IKDC scores greater than 89.9 being 3 times more likely to achieve $90 \%$ limb symmetry. ${ }^{64}$ Patients in the current study reported an average IKDC score of 81.5, which may have negatively influenced quadriceps strength and neuromuscular function. Our results not only indicate that ACL-R patients demonstrated large magnitude deficits in quadriceps strength (isokinetic and isometric) compared to healthy controls, but that large asymmetries ( $\geq$ $15 \%)$ persisted, which may place individuals at a greater risk for joint degeneration. ${ }^{81}$

Bilateral central activation failure (> 10\%) and decreased corticospinal excitability was also observed in ACL-R patients compared to healthy controls. Symmetric quadriceps activation $>99.2 \%$ has been reported to be a strong indicator of good patient-reported function. ${ }^{63}$ Patients in the current study demonstrated very symmetric quadriceps activation despite reporting lower patient-reported function than healthy controls, which suggests this relationship is less true in the presence of clinically meaningful activation failure. 


\section{Involved Limb}

PCA suggested three unique constructs of peripheral, central, and combined peripheral and central muscle function, which conceptually agree with prior theory. Isometric strength assessment is commonly used to evaluate patients after ACL-R; however, isokinetic function explained the greatest variance among all strength measures in this study. Total knee extensor work at $90 \% \mathrm{sec}$ demonstrated the largest magnitude difference between ACL-R patients and healthy controls, and was the best indicator of group membership. Threshold values of knee extensor MVIC torque based on patient-reported function have been established in ACL-R patients. ${ }^{63}$ However, the information gained from this study adds to the current body of literature by establishing a highly sensitive and specific threshold of knee extensor work (18.4 J/kg) to discriminate between ACL-R and healthy individuals, which accounted for $91.6 \%$ of variance in peripheral muscle function. Although isokinetic torque, work, and power were strongly correlated, it is clear that assessment strategies may benefit by including more than isometric strength. As a whole, peripheral muscle function explained nearly $60 \%$ of the variance among all measures of quadriceps function, suggesting this is a highly influential physiological construct to evaluate patients with ACL-R.

Interestingly, H:M ratio and AMT loaded separately from CAR and fatigue index on the involved limb. A clear distinction between the two components was present; however CAR and AMT also loaded to a lesser degree on components one and three respectively. These finding suggest that each combination of measures represent unique, yet interrelated constructs of central and combined peripheral and central muscle function. Spinal reflex excitability may reflect a purely centrally mediated construct, separate from voluntary movement, as it is recorded statically. In contrast, AMT is recorded during voluntary movement, and therefore encompasses a peripheral component, which could explain its secondary loading. AMT demonstrated the greatest magnitude difference between ACL-R patients and healthy controls, and best discriminative ability among measures of central muscle function. Thresholds for AMT have not 
been established previously, however, our results indicated that an AMT $\geq 40 \%$ is moderately sensitive and specific to discern patients with and without ACL-R. Quadriceps CAR loaded highest on combined peripheral and central muscle function, demonstrating the largest magnitude difference between groups, and best discriminatory value. A threshold of $86.4 \%$ for unilateral CAR has been reported to determine good patient-reported function, ${ }^{63}$ however, the higher threshold of $94.7 \%$ reported in this study may result in an improved diagnostic ability. Interestingly, fatigue loaded with CAR and not other measures of peripheral muscle function. Short duration fatiguing protocols are believed to induce fatigue via peripheral mechanisms. However fatigue in the presence of maximal effort tasks may be more reflective of central fatigue that can originate at spinal or supraspinal levels, ${ }^{85}$ which could explain its relationship with CAR. Regardless, each of these components explained $10.7 \%$ of the variance among all measures of quadriceps function, suggesting a meaningful role of centrally mediated constructs.

\section{Uninvolved Limb}

PCA suggested that peripheral (isokinetic strength and knee extension MVIC torque) and combined peripheral and central (AMT, H:M ratio, CAR, and fatigue index) muscle function characterize the uninvolved limb. Persistent deficits in muscle function have been identified in patients at early, mid, and long-term evaluation after ACL-R. The fact that our analysis suggested different constructs for each limb supports the notion that inter-limb differences remain present after ACL-R. Regression analysis in the uninvolved limb was able to classify group membership with $72.8 \%$ accuracy; however, quadriceps function of the involved limb resulted in greater classification accuracy $(83.5 \%)$.

\section{Limb Symmetry}

The results of this study indicate that symmetry of quadriceps neuromuscular function contributes meaningful information to understanding patients after ACL-R. Symmetric peripheral muscle function (isokinetic strength and knee extensor MVIC torque) explained the largest amount of variance among measures of quadriceps function at $54 \%$, followed by combined (CAR 
and fatigue index) at $14.9 \%$, and central muscle function (AMT and H:M ratio) at 10.0\%. However, isokinetic and isometric quadriceps strength were the only tests that differed between limbs in ACL-R patients and also maintained acceptable diagnostic utility (AUC $\geq 0.7$ ). Limb symmetry indices are often used as surrogates of physical impairments, activity limitations, and overall function. ${ }^{71}$ Symmetrical quadriceps strength of $80-90 \%$ is commonly advocated; ${ }^{56}$ however, symmetry of quadriceps neuromuscular function (i.e. spinal and corticospinal excitability) has been examined to a lesser degree. Determining readiness for return to activity after ACL-R is a complex decision that may benefit by including tests that can identify impairments in multiple constructs of muscle function.

\section{Clinical Recommendations}

This was an exploratory analysis conducted to examine the redundancy in information provided, magnitude of group differences, diagnostic, and predictive ability of a given measure(s) of quadriceps function, which may aid the clinician or researcher in selecting the most appropriate test, or test battery in the assessment of ACLR patients. All measures of quadriceps function explained a meaningful degree of variance, and may be considered in assessment paradigms. Isokinetic testing consistently predicted the greatest variance; however, standardized protocols following ACL-R are lacking. ${ }^{86}$ The results of this study suggest that unique, but interrelated constructs of peripheral and centrally mediated muscle function exist in patients with ACL-R. These data appear to support the incorporation of quadriceps neuromuscular function into the test battery, suggesting that strength alone may be insufficient to evaluate clinical outcomes. The authors provide the following three clinical recommendations that may stem from the findings of this study:

- The test battery should include a component of isokinetic knee extensor strength, voluntary activation, and corticospinal excitability.

- From a practical standpoint, knee extension MVIC torque should be assessed because quadriceps central activation ratio is assessed isometrically. 
- Measures of unilateral quadriceps function and symmetry demonstrated relatively good predictive ability, however, using data from the involved limb may be most meaningful in this population.

\section{Limitations}

This was an exploratory analysis, and not confirmatory; therefore, further validation of the proposed test battery is needed. PCA does not account for measurement error and as a result may overestimate the variance explained. Patients with a history of ACL-R in this study spanned a large age range 15-65. While the distribution of age may improve the generalizability of our findings to larger patient populations, this likely increased the heterogeneity among the included sample. To combat this, only patients with primary, unilateral ACL reconstructions were enrolled. Quadriceps neuromuscular function is likely to change over time after ACL-R. The specific thresholds and predictive abilities of tests, or test batteries, may therefore differ based on the time from surgery. The fact that our group was nearly 4 years removed form ACL-R on average, would likely provide a conservative estimate.

\section{CONCLUSION}

Unique constructs of peripheral, central, and combined peripheral and central muscle function are likely to exist in ACL-R patients. The results of this study highlight the redundancy in strength estimates, most notably among strength tests. Total isokinetic knee extensor work at 90\% $/ \mathrm{sec}$, quadriceps CAR, and AMT consistently explained a significant portion of variance in measures of quadriceps function, demonstrated acceptable to excellent diagnostic utility, and predicted group membership with 72.8 to $83.5 \%$ accuracy. Measures of the involved limb appear to have greater diagnostic and predictive ability than the uninvolved limb or limb symmetry. 
Table $1_{\mathrm{iij}}$. Participant demographics and patient-reported outcomes (mean \pm standard deviation)

\begin{tabular}{|c|c|c|c|}
\hline & ACL-R $(n=72)$ & Healthy $(\mathrm{n}=30)$ & $\mathrm{P}$ value \\
\hline Gender ${ }^{a}$ & $32 \mathrm{M}, 40 \mathrm{~F}$ & $12 \mathrm{M}, 18 \mathrm{~F}$ & .680 \\
\hline Age (years) ${ }^{\mathrm{b}}$ & $26.0 \pm 9.3$ & $22.7 \pm 4.6$ & .351 \\
\hline Height $(\mathrm{cm})$ & $172.6 \pm 11.2$ & $174.8 \pm 11.8$ & .396 \\
\hline Mass (kg) & $75.6 \pm 17.7$ & $75.1 \pm 16.2$ & .910 \\
\hline IKDC & $81.5 \pm 14.1$ & $98.2 \pm 4.2$ & $<.001$ \\
\hline KOOS Total ${ }^{\mathrm{b}}$ & $88.2 \pm 9.4$ & $98.7 \pm 2.5$ & $<.001$ \\
\hline KOOS Pain & $90.5 \pm 3.7$ & $98.6 \pm 3.7$ & $<.001$ \\
\hline KOOS Symptoms & $83.9 \pm 12.0$ & $98.0 \pm 4.1$ & $<.001$ \\
\hline KOOS Activities of Daily Living ${ }^{b}$ & $96.01 \pm 6.0$ & $99.8 \pm 0.7$ & $<.001$ \\
\hline KOOS Sport ${ }^{b}$ & $79.0 \pm 21.9$ & $97.8 \pm 6.3$ & $<.001$ \\
\hline KOOS Quality of Life & $68.5 \pm 22.3$ & $96.5 \pm 9.8$ & $<.001$ \\
\hline WOMAC $^{b}$ & $4.9 \pm 5.7$ & $0.3 \pm 0.8$ & $<.001$ \\
\hline WOMAC Pain ${ }^{\mathrm{b}}$ & $19.2 \pm 1.1$ & $19.8 \pm 0.6$ & .003 \\
\hline WOMAC Stiffness ${ }^{\mathrm{b}}$ & $7.0 \pm 1.4$ & $8.0 \pm 0.0$ & $<.001$ \\
\hline WOMAC Function ${ }^{b}$ & $65.3 \pm 4.1$ & $67.9 \pm 0.3$ & $<.001$ \\
\hline VAS: involved $(\mathrm{cm})^{\mathrm{b}}$ & $0.6 \pm 0.8$ & $0.1 \pm 0.2$ & $<.001$ \\
\hline VAS: uninvolved $(\mathrm{cm})^{\mathrm{b}}$ & $0.1 \pm 0.2$ & $0.0 \pm 0.1$ & .063 \\
\hline Tegner: pre-injury & $8.1 \pm 1.4$ & N/A & N/A \\
\hline Tegner: current & $6.3 \pm 1.9$ & $7.2 \pm 1.4$ & .008 \\
\hline Godin leisure-time exercise & $59.1 \pm 25.6$ & $75.5 \pm 29.7$ & .011 \\
\hline Tampa Scale of Kinesiophobia & $33.6 \pm 6.1$ & $28.6 \pm 5.8$ & $<.001$ \\
\hline Veteran's Rand 12-Item Health ${ }^{\mathrm{b}}$ & $79.9 \pm 10.1$ & $86.0 \pm 7.6$ & .005 \\
\hline Time since surgery (months) & $46.5 \pm 58.0$ & N/A & N/A \\
\hline \multirow[t]{3}{*}{ Graft type } & Patellar (40.0\%) & N/A & N/A \\
\hline & Hamstring (47.1\%) & & \\
\hline & Allograft (12.9\%) & & \\
\hline
\end{tabular}

Abbreviations: M, male; F, female; IKDC, International Knee Documentation Committee; KOOS, Knee Injury and Osteoarthritis Score; WOMAC, Western Ontario and McMaster Universities Arthritis Index;

VAS, visual analog scale

${ }^{\text {a }}$ Chi-squared

${ }^{\mathrm{b}}$ Mann-Whitney U

Alpha level set at $p \leq .05$ 
Table $2_{\mathrm{iii}}$. Quadriceps neuromuscular function in ACL-R patients and healthy individuals (mean \pm standard deviation)

\begin{tabular}{|c|c|c|c|c|c|c|}
\hline & \multicolumn{6}{|c|}{ Group } \\
\hline & \multicolumn{3}{|c|}{ ACL-R } & \multicolumn{3}{|c|}{ Healthy } \\
\hline & Involved & Uninvolved & $\begin{array}{c}\text { Limb } \\
\text { Symmetry }\end{array}$ & $\begin{array}{l}\text { Matched } \\
\text { Involved }\end{array}$ & $\begin{array}{c}\text { Matched } \\
\text { Uninvolved }\end{array}$ & $\begin{array}{c}\text { Limb } \\
\text { Symmetry }\end{array}$ \\
\hline Peak torque $\left(90^{\circ} / \mathrm{s}\right)(\mathrm{Nm} / \mathrm{kg})$ & $1.7 \pm 0.5^{*}$ & $2.1 \pm 0.5$ & $0.82 \pm 0.19 \dagger$ & $2.2 \pm 0.4$ & $2.2 \pm 0.6$ & $1.29 \pm 1.64$ \\
\hline Peak torque $\left(180^{\circ} / \mathrm{s}\right)(\mathrm{Nm} / \mathrm{kg})$ & $1.3 \pm 0.4^{*}$ & $1.6 \pm 0.4$ & $0.83 \pm 0.16 \dagger$ & $1.7 \pm 0.3$ & $1.7 \pm 0.3$ & $1.01 \pm 0.15$ \\
\hline Total work $\left(90^{\circ} / \mathrm{s}\right)(\mathrm{J} / \mathrm{kg})$ & $14.7 \pm 4.5^{*}$ & $18.0 \pm 4.5^{*}$ & $0.82 \pm 0.16 \dagger$ & $20.1 \pm 3.4$ & $20.5 \pm 3.6$ & $1.00 \pm 0.18$ \\
\hline Total work $\left(180^{\circ} / \mathrm{s}\right)(\mathrm{J} / \mathrm{kg})$ & $11.5 \pm 3.5^{*}$ & $13.9 \pm 3.7^{*}$ & $0.84 \pm 0.16 \dagger$ & $15.8 \pm 2.9$ & $15.8 \pm 3.2$ & $1.02 \pm 0.22$ \\
\hline Average power $\left(90^{\circ} / \mathrm{s}\right)(\mathrm{W} / \mathrm{kg})$ & $1.6 \pm 0.5^{*}$ & $2.0 \pm 0.5^{*}$ & $0.83 \pm 0.17 \dagger$ & $2.2 \pm 0.4$ & $2.2 \pm 0.4$ & $1.00 \pm 0.22$ \\
\hline Average power $\left(180^{\circ} / \mathrm{s}\right)(\mathrm{W} / \mathrm{kg})$ & $2.1 \pm 0.6^{*}$ & $2.6 \pm 0.7^{*}$ & $0.84 \pm 0.17 \dagger$ & $2.9 \pm 0.6$ & $2.9 \pm 0.6$ & $1.02 \pm 0.21$ \\
\hline MVIC torque $(\mathrm{Nm} / \mathrm{kg})$ & $2.0 \pm 0.6^{*}$ & $2.4 \pm 0.7^{*}$ & $0.85 \pm 0.20 \dagger$ & $2.8 \pm 0.6$ & $2.7 \pm 0.6$ & $1.04 \pm 0.14$ \\
\hline Fatigue index $(\%)$ & $18.2 \pm 9.2$ & $20.2 \pm 7.9$ & $1.98 \pm 7.96 \dagger$ & $21.8 \pm 8.1$ & $22.2 \pm 8.2$ & $0.76 \pm 6.19$ \\
\hline Central activation ratio $(\%)$ & $88.4 \pm 10.1^{*}$ & $89.8 \pm 9.6^{*}$ & $0.99 \pm 0.08$ & $95.2 \pm 5.6$ & $93.8 \pm 6.7$ & $1.02 \pm 0.06$ \\
\hline Hoffmann reflex (H:M) & $0.21 \pm 0.19^{*}$ & $0.18 \pm 0.17$ & $1.27 \pm 0.89$ & $0.14 \pm 0.12$ & $0.14 \pm 0.12$ & $1.11 \pm 0.66$ \\
\hline Active motor threshold $(\% \mathrm{~T})$ & $45.2 \pm 8.6^{*}$ & $44.3 \pm 8.4^{*}$ & $0.99 \pm 0.15$ & $39.0 \pm 4.1$ & $39.0 \pm 3.4$ & $1.01 \pm 0.12$ \\
\hline
\end{tabular}

* Different from healthy

† Different between limbs

Alpha level set at $p \leq .05$ 
Table $3_{\mathrm{iii}}$. Principal component analysis using involved limb measures of quadriceps function

\begin{tabular}{|c|c|c|c|c|c|c|}
\hline \multirow[b]{2}{*}{ Variable } & \multicolumn{3}{|c|}{ Component $^{\mathrm{a}}$} & \multicolumn{3}{|c|}{ Criteria for Retention } \\
\hline & 1 & 2 & 3 & $\begin{array}{c}\text { Different from } \\
\text { healthy }(p)\end{array}$ & $\begin{array}{l}\text { Magnitude of } \\
\text { difference }(d)\end{array}$ & $\begin{array}{c}\text { Diagnostic } \\
\text { ability (AUC) }\end{array}$ \\
\hline Average power $\left(180^{\circ} / \mathrm{s}\right)$ & .971 & & & .001 & 1.33 & .812 \\
\hline Peak torque $\left(180^{\circ} / \mathrm{s}\right)$ & .967 & & & .001 & 1.33 & .806 \\
\hline Average power $\left(90^{\circ} / \mathrm{s}\right)$ & .967 & & & .001 & 1.50 & .833 \\
\hline Total work $\left(90^{\circ} / \mathrm{s}\right)$ & .957 & & & .001 & 1.59 & .847 \\
\hline Total work $\left(180^{\circ} / \mathrm{s}\right)$ & .954 & & & .001 & 1.48 & .832 \\
\hline Peak torque $\left(90^{\circ} / \mathrm{s}\right)$ & .954 & & & .001 & 1.25 & .826 \\
\hline MVIC torque & .863 & & & .001 & 1.33 & .821 \\
\hline Hoffmann reflex & & .813 & & .047 & 0.58 & .590 \\
\hline Active motor threshold & & .687 & & .001 & 1.51 & .730 \\
\hline Central activation ratio & & & .752 & .000 & 1.21 & .731 \\
\hline Fatigue index & & & .629 & .056 & 0.44 & .635 \\
\hline
\end{tabular}

Principal component analysis was used as the method of extraction

Variables listed in order of strength of loading

Missing data replaced: H:M $(n=4)$, AMT $(n=2)$

${ }^{\text {a }}$ Factor matrix coefficients following Varimax rotation with Kaiser normalization

$\mathrm{KMO}=.822 ;$ Bartlett's $\chi^{2}(55)=917.5, p<.001$

$1-$ Eigenvalue $=6.5, \%$ variance $=59.1$

$2-$ Eigenvalue $=1.2, \%$ variance $=10.7$

$3-$ Eigenvalue $=1.2, \%$ variance $=10.7$

Cumulative variance $=80.5 \%$ 
Table $4_{\mathrm{iij}}$. Principal component analysis using uninvolved limb measures of quadriceps function

\begin{tabular}{|c|c|c|c|c|c|}
\hline \multirow[b]{2}{*}{ Variable } & \multicolumn{2}{|c|}{ Component $^{\mathrm{a}}$} & \multicolumn{3}{|c|}{ Criteria for Retention } \\
\hline & 1 & 2 & $\begin{array}{c}\text { Different from } \\
\text { healthy }(p)\end{array}$ & $\begin{array}{l}\text { Magnitude of } \\
\text { difference }(d)\end{array}$ & $\begin{array}{c}\text { Diagnostic } \\
\text { ability (AUC) }\end{array}$ \\
\hline Average power $\left(180^{\circ} / \mathrm{s}\right)$ & .957 & & .028 & 0.50 & .631 \\
\hline Peak torque $\left(180^{\circ} / \mathrm{s}\right)$ & .956 & & .086 & 0.33 & .592 \\
\hline Total work $\left(90^{\circ} / \mathrm{s}\right)$ & .952 & & .008 & 0.69 & .684 \\
\hline Total work $\left(180^{\circ} / \mathrm{s}\right)$ & .948 & & .018 & 0.59 & .650 \\
\hline Peak torque $\left(90^{\circ} / \mathrm{s}\right)$ & .947 & & .105 & 0.17 & .632 \\
\hline Average power $\left(90^{\circ} / \mathrm{s}\right)$ & .937 & & .018 & 0.50 & .663 \\
\hline MVIC torque & .814 & & .031 & 0.50 & .626 \\
\hline Active motor threshold & & .696 & .005 & 1.56 & .732 \\
\hline Hoffmann reflex & & .632 & .311 & 0.33 & .547 \\
\hline Central activation ratio & & .571 & .019 & 0.60 & .640 \\
\hline Fatigue index & & .569 & .264 & 0.24 & .580 \\
\hline
\end{tabular}

Principal component analysis was used as the method of extraction

Variables listed in order of strength of loading

Missing data replaced: H:M $(n=4)$, AMT $(n=3)$

${ }^{a}$ Factor matrix coefficients following Varimax rotation with Kaiser normalization

KMO $=.804 ;$ Bartlett's $\chi^{2}(55)=819.3, p<.001$

$1-$ Eigenvalue $=6.2, \%$ variance $=56.1$

$2-$ Eigenvalue $=1.6, \%$ variance $=14.6$

Cumulative variance $=70.7 \%$ 
Table $5_{\mathrm{ij}}$. Principal component analysis using limb symmetry measures of quadriceps function

\begin{tabular}{|c|c|c|c|c|c|c|}
\hline \multirow[b]{2}{*}{ Variable } & \multicolumn{3}{|c|}{ Component $^{\mathrm{a}}$} & \multicolumn{3}{|c|}{ Criteria for Retention } \\
\hline & 1 & 2 & 3 & $\begin{array}{l}\text { Between limb } \\
\text { difference }(p)\end{array}$ & $\begin{array}{l}\text { Magnitude of } \\
\text { difference }(d)\end{array}$ & $\begin{array}{c}\text { Diagnostic } \\
\text { ability (AUC) }\end{array}$ \\
\hline Average power $\left(180^{\circ} / \mathrm{s}\right)$ & .956 & & & .001 & 0.66 & .778 \\
\hline Total work $\left(180^{\circ} / \mathrm{s}\right)$ & .949 & & & .001 & 0.67 & .787 \\
\hline Peak torque $\left(180^{\circ} / \mathrm{s}\right)$ & .947 & & & .001 & 0.75 & .808 \\
\hline Average power $\left(90^{\circ} / \mathrm{s}\right)$ & .944 & & & .001 & 0.77 & .743 \\
\hline Total work $\left(90^{\circ} / \mathrm{s}\right)$ & .934 & & & .001 & 0.75 & .774 \\
\hline Peak torque $(90 \% / \mathrm{s})$ & .928 & & & .001 & 0.82 & .746 \\
\hline MVIC torque & .717 & & & .001 & 0.60 & .776 \\
\hline Fatigue index & & .812 & & .038 & 0.35 & .529 \\
\hline Central activation ratio & & .745 & & .087 & 0.15 & .610 \\
\hline Active motor threshold & & & .948 & .279 & 0.10 & .558 \\
\hline Hoffmann reflex & & & .321 & .137 & 0.11 & .530 \\
\hline
\end{tabular}

Principal component analysis was used as the method of extraction

Variables listed in order of strength of loading

Missing data replaced: H:M ( $=6)$, AMT $(n=3)$

${ }^{a}$ Factor matrix coefficients following Varimax rotation with Kaiser normalization

$\mathrm{KMO}=.877 ;$ Bartlett's $\chi^{2}(55)=849.9, p<.001$

$1-$ Eigenvalue $=5.9, \%$ variance $=54.0$

$2-$ Eigenvalue $=1.6, \%$ variance $=14.9$

3 - Eigenvalue $=1.1, \%$ variance $=10.0$

Cumulative variance $=78.9 \%$ 
Table $6_{\mathrm{iij}}$. Principal component analysis using patient-reported outcomes

\begin{tabular}{|c|c|c|c|c|c|}
\hline \multirow[b]{2}{*}{ Variable } & \multicolumn{2}{|c|}{ Component $^{\mathrm{a}}$} & \multicolumn{3}{|c|}{ Criteria for Retention } \\
\hline & 1 & 2 & $\begin{array}{c}\text { Different from } \\
\text { healthy }(p)\end{array}$ & $\begin{array}{l}\text { Magnitude of } \\
\text { difference }(d)\end{array}$ & $\begin{array}{c}\text { Diagnostic } \\
\text { ability (AUC) }\end{array}$ \\
\hline KOOS Total & 934 & & .001 & 4.28 & .919 \\
\hline IKDC Subjective & .860 & & .001 & 3.99 & .923 \\
\hline WOMAC Total & .858 & & .001 & 5.74 & .822 \\
\hline Tampa Scale of Kinesiophobia & .542 & & .001 & 0.86 & .707 \\
\hline Godin Leisure-Time Activity & & .866 & .004 & 0.55 & .647 \\
\hline Tegner Activity Scale & & .757 & .006 & 0.66 & .654 \\
\hline Veteran's Rand 12-Item Health Survey & & .623 & .018 & 0.79 & 677 \\
\hline
\end{tabular}

Principal component analysis was used as the method of extraction

Variables listed in order of strength of loading

${ }^{\text {a }}$ Factor matrix coefficients following Varimax rotation with Kaiser normalization

$\mathrm{KMO}=.771$; Bartlett's $\chi^{2}(21)=299.4, p<.001$

$1-$ Eigenvalue $=3.0, \%$ variance $=43.7$

$2-$ Eigenvalue $=1.9, \%$ variance $=27.0$

Cumulative variance $=70.7 \%$ 
Table $7_{\text {iii }}$. Results from logistic regression analyses to predict group membership

\begin{tabular}{lccc}
\hline & Involved Limb $^{\mathrm{a}}$ & Uninvolved Limb $^{\text {b }}$ & Limb Symmetry $^{{ }^{c}}$ \\
\hline Accuracy of classification (\%) & 83.5 & 72.8 & 79.5 \\
Sensitivity (\%) & 91.4 & 91.3 & 97.1 \\
Specificity (\%) & 57.1 & 17.4 & 15.8 \\
Positive predictive value (\%) & 87.7 & 75.9 & 80.7 \\
Negative predictive value (\%) & 66.7 & 40.0 & 60.0 \\
Positive likelihood ratio & 2.13 & 1.11 & 1.15 \\
Negative likelihood ratio & 0.15 & 0.50 & 0.18 \\
$\mathrm{R}^{2}$ & .490 & .220 & .309 \\
$P$ value & $<.001$ & .001 & $<.001$ \\
\hline
\end{tabular}

${ }^{a}$ Model includes: total work at $90^{\circ} / \mathrm{sec}^{*}$, AMT $\dagger$, and CAR*

${ }^{\mathrm{b}}$ Model includes: total work at $90 \% \mathrm{sec}^{*}$, and AMT*

${ }^{c}$ Model includes: peak torque symmetry at $180^{\circ} / \mathrm{sec}^{*}$, CAR symmetry, and AMT symmetry

* Significant at $p \leq .05$

$\dagger$ Significant at $p \leq .10$ 


\section{REFERENCES}

1. Griffin LY, Albohm MJ, Arendt EA, et al. Understanding and preventing noncontact anterior cruciate ligament injuries: a review of the Hunt Valley II meeting, January 2005. Am J Sport Med. Sep 2006;34(9):1512-1532.

2. Hunter DJ, Lohmander LS, Makovey J, et al. The effect of anterior cruciate ligament injury on bone curvature: exploratory analysis in the KANON trial. Osteoarthritis Cartilage. Jul 2014;22(7):959-968.

3. Shea KG, Carey JL, Richmond J, et al. The American Academy of Orthopaedic Surgeons evidence-based guideline on management of anterior cruciate ligament injuries. J Bone Joint Surg Am. Apr 15 2015;97(8):672-674.

4. Luc B, Gribble PA, Pietrosimone BG. Osteoarthritis prevalence following anterior cruciate ligament reconstruction: a systematic review and numbers-needed-to-treat analysis. $J$ Athl Train. Nov-Dec 2014;49(6):806-819.

5. Ardern CL, Taylor NF, Feller JA, Webster KE. Fifty-five per cent return to competitive sport following anterior cruciate ligament reconstruction surgery: an updated systematic review and meta-analysis including aspects of physical functioning and contextual factors. $\mathrm{Br} \mathrm{J}$ Sports Med. Nov 2014;48(21):1543-1552.

6. Wiggins AJ, Grandhi RK, Schneider DK, Stanfield D, Webster KE, Myer GD. Risk of Secondary Injury in Younger Athletes After Anterior Cruciate Ligament Reconstruction: A Systematic Review and Meta-analysis. Am J Sport Med. Jan 152016.

7. Oiestad BE, Juhl CB, Eitzen I, Thorlund JB. Knee extensor muscle weakness is a risk factor for development of knee osteoarthritis. A systematic review and meta-analysis. Osteoarthritis Cartilage. Feb 2015;23(2):171-177.

8. Hart JM, Pietrosimone B, Hertel J, Ingersoll CD. Quadriceps activation following knee injuries: a systematic review. J Athl Train. Jan-Feb 2010;45(1):87-97.

9. Pietrosimone $\mathrm{B}$, Thomas $\mathrm{AC}$, Saliba SA, Ingersoll CD. Association between quadriceps strength and self-reported physical activity in people with knee osteoarthritis. Int J Sports Phys Ther. May 2014;9(3):320-328.

10. Pietrosimone BG, Lepley AS, Ericksen HM, Gribble PA, Levine J. Quadriceps strength and corticospinal excitability as predictors of disability after anterior cruciate ligament reconstruction. J Sport Rehabil. Feb 2013;22(1):1-6.

11. Tourville TW, Jarrell KM, Naud S, Slauterbeck JR, Johnson RJ, Beynnon BD. Relationship between isokinetic strength and tibiofemoral joint space width changes after anterior cruciate ligament reconstruction. Am J Sport Med. Feb 2014;42(2):302-311.

12. Pelletier JP, Raynauld JP, Berthiaume MJ, et al. Risk factors associated with the loss of cartilage volume on weight-bearing areas in knee osteoarthritis patients assessed by quantitative magnetic resonance imaging: a longitudinal study. Arthritis Res Ther. 2007;9(4):R74.

13. Pietrosimone BG, Lepley AS, Ericksen HM, Clements A, Sohn DH, Gribble PA. Neural Excitability Alterations After Anterior Cruciate Ligament Reconstruction. J Athl Train. Jun 2015;50(6):665-674.

14. Rice DA, McNair PJ. Quadriceps arthrogenic muscle inhibition: neural mechanisms and treatment perspectives. Semin Arthritis Rheum. Dec 2010;40(3):250-266. 
15. Lepley AS, Gribble PA, Thomas AC, Tevald MA, Sohn DH, Pietrosimone BG. Quadriceps neural alterations in anterior cruciate ligament reconstructed patients: A 6-month longitudinal investigation. Scan J Med Sci Sports. Feb 182015.

16. Kuenze CM, Hertel J, Weltman A, Diduch D, Saliba SA, Hart JM. Persistent Neuromuscular and Corticomotor Quadriceps Asymmetry After Anterior Cruciate Ligament Reconstruction. J Athl Train. Jan 262015.

17. Pietrosimone BG, Hertel J, Ingersoll CD, Hart JM, Saliba SA. Voluntary quadriceps activation deficits in patients with tibiofemoral osteoarthritis: a meta-analysis. $P M R$. Feb 2011;3(2):153-162; quiz 162.

18. Ward S, Pearce AJ, Pietrosimone B, Bennell K, Clark R, Bryant AL. Neuromuscular deficits after peripheral joint injury: a neurophysiological hypothesis. Muscle Nerve. Mar 2015;51(3):327-332.

19. Hopkins JT, Ingersoll CD. Arthrogenic Muscle Inhibition: A Limiting Factor in Joint Rehabilitation (Book). J Sport Rehabil. 2000;9(2):135.

20. Hopkins JT, Ingersoll CD, Krause BA, Edwards JE, Cordova ML. Effect of knee joint effusion on quadriceps and soleus motoneuron pool excitability. Med Sci Sports Exerc. Jan 2001;33(1):123-126.

21. Lepley AS, Bahhur NO, Murray AM, Pietrosimone BG. Quadriceps corticomotor excitability following an experimental knee joint effusion. Knee Surg Sports Traumatol Arthrosc. Dec 11 2013.

22. Baumeister J, Reinecke K, Schubert M, Weiss M. Altered electrocortical brain activity after ACL reconstruction during force control. J Orthop Res. Sep 2011;29(9):1383-1389.

23. Rossi S, Hallett M, Rossini PM, Pascual-Leone A, Safety of TMSCG. Safety, ethical considerations, and application guidelines for the use of transcranial magnetic stimulation in clinical practice and research. Clin Neurophysiol. Dec 2009;120(12):2008-2039.

24. Grevnerts HT, Terwee CB, Kvist J. The measurement properties of the IKDC-subjective knee form. Knee Surg Sports Traumatol Arthrosc. Dec 2015;23(12):3698-3706.

25. Roos EM, Roos HP, Lohmander LS, Ekdahl C, Beynnon BD. Knee Injury and Osteoarthritis Outcome Score (KOOS)--development of a self-administered outcome measure. J Orthop Sports Phys Ther. Aug 1998;28(2):88-96.

26. McConnell S, Kolopack P, Davis AM. The Western Ontario and McMaster Universities Osteoarthritis Index (WOMAC): a review of its utility and measurement properties. Arthritis Rheum. Oct 2001;45(5):453-461.

27. Briggs KK, Lysholm J, Tegner Y, Rodkey WG, Kocher MS, Steadman JR. The reliability, validity, and responsiveness of the Lysholm score and Tegner activity scale for anterior cruciate ligament injuries of the knee: 25 years later. Am J Sport Med. May 2009;37(5):890897.

28. French DJ, France CR, Vigneau F, French JA, Evans RT. Fear of movement/(re)injury in chronic pain: a psychometric assessment of the original English version of the Tampa scale for kinesiophobia (TSK). Pain. Jan 2007;127(1-2):42-51.

29. Selim AJ, Rogers W, Qian SX, Brazier J, Kazis LE. A preference-based measure of health: the VR-6D derived from the veterans RAND 12-Item Health Survey. Qual Life Res. Oct 2011;20(8):1337-1347.

30. Norte GE, Saliba SA, Hart JM. Immediate Effects of Therapeutic Ultrasound on Quadriceps Spinal Reflex Excitability in Patients With Knee Injury. Arch Ohys Med Rehabil. Sep 2015;96(9):1591-1598.

31. Merletti R, Hermens H. Introduction to the special issue on the SENIAM European Concerted Action. J Electromyogr Kinesiol. Oct 2000;10(5):283-286.

32. Palmieri RM, Ingersoll CD, Hoffman MA. The hoffmann reflex: methodologic considerations and applications for use in sports medicine and athletic training research. $J$ Athl Train. Jul 2004;39(3):268-277. 
33. Hart JM, Fritz JM, Kerrigan DC, Saliba EN, Gansneder BM, Ingersoll CD. Reduced quadriceps activation after lumbar paraspinal fatiguing exercise. J Athl Train. Jan-Mar 2006;41(1):79-86.

34. Kent-Braun JA, Le Blanc R. Quantitation of central activation failure during maximal voluntary contractions in humans. Muscle Nerve. Jul 1996;19(7):861-869.

35. Surakka J, Romberg A, Ruutiainen J, Virtanen A, Aunola S, Maentaka K. Assessment of muscle strength and motor fatigue with a knee dynamometer in subjects with multiple sclerosis: a new fatigue index. Clin Rehabil. Sep 2004;18(6):652-659.

36. Norte GE, Pietrosimone BG, Hart JM, Hertel J, Ingersoll CD. Relationship between transcranial magnetic stimulation and percutaneous electrical stimulation in determining the quadriceps central activation ratio. Am J Phys Med Rehabil. Dec 2010;89(12):986-996.

37. Wolf SL, Butler AJ, Campana GI, et al. Intra-subject reliability of parameters contributing to maps generated by transcranial magnetic stimulation in able-bodied adults. Clin Neurophysiol. Aug 2004;115(8):1740-1747.

38. Park J, Hopkins JT. Within- and between-session reliability of the maximal voluntary knee extension torque and activation. Int J Neurosci. Jan 2013;123(1):55-59.

39. Poulsen JB, Rose MH, Moller K, Perner A, Jensen BR. A Novel Noninvasive Method for Measuring Fatigability of the Quadriceps Muscle in Noncooperating Healthy Subjects. Biomed Res Int. 2015;2015:193493.

40. Hopkins JT, Wagie NC. Intrasession and intersession reliability of the quadriceps Hoffmann reflex. Electromyogr Clin Neurophysiol. Mar 2003;43(2):85-89.

41. Luc BA, Lepley AS, Tevald MA, Gribble PA, White DB, Pietrosimone BG. Reliability of Corticomotor Excitability in Leg and Thigh Musculature at 14 and 28 Days. J Sport Rehabil. Oct 12013.

42. Ingersoll CD, Palmieri RM, Hopkins JT. A joint dilemma. Rehab Manag. Jan-Feb 2003;16(1):38-42.

43. Kilic BA, Dingil O, Erkula G, Elmas C, Erdogan D, Atik OS. Evaluation of the muscles around the knee in rabbits whose anterior cruciate and/or medial collateral ligaments were dissected. Arch Orthop Trauma Surg. Nov 2004;124(9):626-630.

44. Hart JM, Bessette M, Choi L, Hogan MV, Diduch D. Sensory response following knee joint damage in rabbits. BMC Musculoskelet Disord. 2014;15:139.

45. Kuenze C, Hertel J, Hart JM. Effects of exercise on lower extremity muscle function after anterior cruciate ligament reconstruction. J Sport Rehabil. Feb 2013;22(1):33-40.

46. Snyder-Mackler L, Binder-Macleod SA, Williams PR. Fatigability of human quadriceps femoris muscle following anterior cruciate ligament reconstruction. Med Sci Sports Exerc. Jul 1993;25(7):783-789.

47. Lopresti C, Kirkendall DT, Street GM, Dudley AW. Quadriceps Insufficiency following Repair of the Anterior Cruciate Ligament. J Orthop Sports Phys Ther. 1988;9(7):245-249.

48. Edstrom L. Selective atrophy of red muscle fibres in the quadriceps in long-standing kneejoint dysfunction. Injuries to the anterior cruciate ligament. J Neurol Sci. Dec 1970;11(6):551-558.

49. Lorentzon R, Elmqvist LG, Sjostrom M, Fagerlund M, Fuglmeyer AR. Thigh musculature in relation to chronic anterior cruciate ligament tear: muscle size, morphology, and mechanical output before reconstruction. Am J Sport Med. May-Jun 1989;17(3):423-429.

50. Thomas AC, Wojtys EM, Brandon C, Palmieri-Smith RM. Muscle atrophy contributes to quadriceps weakness after anterior cruciate ligament reconstruction. J Sci Med Sport. Jan 2016;19(1):7-11.

51. Krishnan C, Williams GN. Factors explaining chronic knee extensor strength deficits after ACL reconstruction. J Orthop Res. May 2011;29(5):633-640. 
52. Shanahan CJ, Hodges PW, Wrigley TV, Bennell KL, Farrell MJ. Organisation of the motor cortex differs between people with and without knee osteoarthritis. Arthritis Res Ther. 2015;17:164.

53. Booth FW, Weeden SH, Tseng BS. Effect of aging on human skeletal muscle and motor function. Med Sci Sports Exerc. May 1994;26(5):556-560.

54. Rogers MA, Evans WJ. Changes in skeletal muscle with aging: effects of exercise training. Exerc Sport Sci Rev. 1993;21:65-102.

55. Larsson L. Motor units: remodeling in aged animals. J Gerontol A Biol Sci Med Sci. Nov 1995;50 Spec No:91-95.

56. Barber-Westin SD, Noyes FR. Factors used to determine return to unrestricted sports activities after anterior cruciate ligament reconstruction. Arthroscopy. Dec 2011;27(12):16971705.

57. Risberg MA, Oiestad BE, Gunderson R, et al. Changes in Knee Osteoarthritis, Symptoms, and Function After Anterior Cruciate Ligament Reconstruction: A 20-Year Prospective Follow-up Study. Am J Sport Med. Feb 242016.

58. Ardern CL, Taylor NF, Feller JA, Webster KE. Return-to-sport outcomes at 2 to 7 years after anterior cruciate ligament reconstruction surgery. Am J Sport Med. Jan 2012;40(1):41-48.

59. Filbay SR, Crossley KM, Ackerman IN. Activity preferences, lifestyle modifications and reinjury fears influence longer-term quality of life in people with knee symptoms following anterior cruciate ligament reconstruction: a qualitative study. J Physiother. Mar 162016.

60. Tengman E, Brax Olofsson L, Nilsson KG, Tegner Y, Lundgren L, Hager CK. Anterior cruciate ligament injury after more than 20 years: I. Physical activity level and knee function. Scan J Med Sci Sports. Dec 2014;24(6):e491-500.

61. Lynch AD, Logerstedt DS, Grindem H, et al. Consensus criteria for defining 'successful outcome' after ACL injury and reconstruction: a Delaware-Oslo ACL cohort investigation. $\mathrm{Br}$ J Sports Med. Mar 2015;49(5):335-342.

62. Myer GD, Martin L, Jr., Ford KR, et al. No association of time from surgery with functional deficits in athletes after anterior cruciate ligament reconstruction: evidence for objective return-to-sport criteria. Am J Sport Med. Oct 2012;40(10):2256-2263.

63. Kuenze C, Hertel J, Saliba S, Diduch DR, Weltman A, Hart JM. Clinical thresholds for quadriceps assessment after anterior cruciate ligament reconstruction. J Sport Rehabil. Feb 2015;24(1):36-46.

64. Zwolski C, Schmitt LC, Quatman-Yates C, Thomas S, Hewett TE, Paterno MV. The Influence of Quadriceps Strength Asymmetry on Patient-Reported Function at Time of Return to Sport After Anterior Cruciate Ligament Reconstruction. Am J Sport Med. Jul 16 2015.

65. Goetschius J, Hart JM. Knee-Extension Torque Variability and Subjective Knee Function in Patients With a History of Anterior Cruciate Ligament Reconstruction. J Athl Train. Jan 2016;51(1):22-27.

66. Paterno MV, Rauh MJ, Schmitt LC, Ford KR, Hewett TE. Incidence of Second ACL Injuries 2 Years After Primary ACL Reconstruction and Return to Sport. Am J Sport Med. Apr 21 2014;42(7):1567-1573.

67. Shapiro ET, Richmond JC, Rockett SE, McGrath MM, Donaldson WR. The use of a generic, patient-based health assessment (SF-36) for evaluation of patients with anterior cruciate ligament injuries. Am J Sport Med. Mar-Apr 1996;24(2):196-200.

68. Roberts D, Kuenze C, Saliba S, Hart JM. Accessory muscle activation during the superimposed burst technique. J Electromyogr Kinesiol. Aug 2012;22(4):540-545.

69. Schmitt LC, Paterno MV, Ford KR, Myer GD, Hewett TE. Strength Asymmetry and Landing Mechanics at Return to Sport after Anterior Cruciate Ligament Reconstruction. Med Sci Sports Exerc. Jul 2015;47(7):1426-1434. 
70. Schmitt LC, Paterno MV, Hewett TE. The impact of quadriceps femoris strength asymmetry on functional performance at return to sport following anterior cruciate ligament reconstruction. J Orthop Sports Phys Ther. Sep 2012;42(9):750-759.

71. Logerstedt D, Arundale A, Lynch A, Snyder-Mackler L. A conceptual framework for a sports knee injury performance profile (SKIPP) and return to activity criteria (RTAC). Braz J Phys Ther. Oct 62015.

72. Lepley LK. Deficits in Quadriceps Strength and Patient-Oriented Outcomes at Return to Activity After ACL Reconstruction: A Review of the Current Literature. Sports Health. May 2015;7(3):231-238.

73. Baumeister J, Reinecke K, Weiss M. Changed cortical activity after anterior cruciate ligament reconstruction in a joint position paradigm: an EEG study. Scan J Med Sci Sports. Aug 2008;18(4):473-484.

74. Tengman E, Brax Olofsson L, Stensdotter AK, Nilsson KG, Hager CK. Anterior cruciate ligament injury after more than 20 years. II. Concentric and eccentric knee muscle strength. Scan J Med Sci Sports. Dec 2014;24(6):e501-509.

75. Harput G, Kilinc HE, Ozer H, Baltaci G, Mattacola CG. Quadriceps and Hamstring Strength Recovery During Early Neuromuscular Rehabilitation After ACL Hamstring-Tendon Autograft Reconstruction. J Sport Rehabil. Nov 2015;24(4):398-404.

76. Iriuchishima T, Shirakura K, Horaguchi T, et al. Age as a predictor of residual muscle weakness after anterior cruciate ligament reconstruction. Knee Surg Sports Traumatol Arthrosc. Jan 2012;20(1):173-178.

77. Serrao PR, Gramani-Say K, Lessi GC, Mattiello SM. Knee extensor torque of men with early degrees of osteoarthritis is associated with pain, stiffness and function. Rev Bras Fisioter. JulAug 2012;16(4):289-294.

78. Lewek MD, Rudolph KS, Snyder-Mackler L. Quadriceps femoris muscle weakness and activation failure in patients with symptomatic knee osteoarthritis. J Orthop Res. Jan 2004;22(1):110-115.

79. Petersen W, Taheri P, Forkel P, Zantop T. Return to play following ACL reconstruction: a systematic review about strength deficits. Arch Orthop Trauma Surg. Oct 2014;134(10):1417-1428.

80. Ithurburn MP, Paterno MV, Ford KR, Hewett TE, Schmitt LC. Young Athletes With Quadriceps Femoris Strength Asymmetry at Return to Sport After Anterior Cruciate Ligament Reconstruction Demonstrate Asymmetric Single-Leg Drop-Landing Mechanics. Am J Sport Med. Nov 2015;43(11):2727-2737.

81. Palmieri-Smith RM, Lepley LK. Quadriceps Strength Asymmetry After Anterior Cruciate Ligament Reconstruction Alters Knee Joint Biomechanics and Functional Performance at Time of Return to Activity. Am J Sport Med. Jul 2015;43(7):1662-1669.

82. Urbach D, Nebelung W, Weiler HT, Awiszus F. Bilateral deficit of voluntary quadriceps muscle activation after unilateral ACL tear. Med Sci Sports Exerc. Dec 1999;31(12):16911696.

83. Amireault S, Godin G. The Godin-Shephard leisure-time physical activity questionnaire: validity evidence supporting its use for classifying healthy adults into active and insufficiently active categories. Percept Mot Skills. Apr 2015;120(2):604-622.

84. Groppa $S$, Oliviero A, Eisen A, et al. A practical guide to diagnostic transcranial magnetic stimulation: report of an IFCN committee. Clinical Neurophysiol. May 2012;123(5):858-882.

85. Gandevia SC. Spinal and supraspinal factors in human muscle fatigue. Physiol Rev. Oct 2001;81(4):1725-1789.

86. Undheim MB, Cosgrave $\mathrm{C}$, King $\mathrm{E}$, et al. Isokinetic muscle strength and readiness to return to sport following anterior cruciate ligament reconstruction: is there an association? A systematic review and a protocol recommendation. Br J Sports Med. Oct 2015;49(20):13051310. 
SECTION III: APPENDICES 


\section{APPENDIX A}

\section{The Problem}

\section{Significance}

Knee joint injuries remain common among athletic and recreationally active populations. Unfortunately, early mal-adaptations throughout the central nervous system can occur following injury, resulting in long-term consequences with regard to joint health and decreased quality of life. Anterior cruciate ligament injuries present a specific challenge to joint health in this regard and continue to constitute a large portion of major knee joint injuries in young, active populations. Post-traumatic muscle dysfunction is well described following ACL reconstruction, ${ }^{1}$ and is linked to a sequelae of impairments detrimental to global and joint-specific health, including decreased physical activity, ${ }^{2-5}$ increased risk of re-injury, ${ }^{6}$ and an accelerated onset of post-traumatic knee joint osteoarthritis. ${ }^{7-11}$ Strong evidence exists supporting the causal link between ACL-R and early post-traumatic articular cartilage joint degeneration. ${ }^{9,12-14}$ Since articular cartilage degeneration is irreversible, the hallmark for prevention is early detection.

The ability of the central nervous system to reorganize, adapt, and compensate is theorized to be a compensatory mechanism, thereby preserving global functional capabilities in the presence of peripheral injury. ${ }^{15}$ Regional brain activity has been studied using functional MRI imaging, demonstrating central re-organization of the somatosensory cortex in patients with a recent history of ACL injury ${ }^{16}$ and reconstruction. ${ }^{17}$ Central and peripheral neural adaptations have been identified following ACL injury, and hypothesized to contribute to post-traumatic muscle impairments. ${ }^{18,19}$ Such changes may arise from peripheral (muscle), spinal (spinal cord), or supraspinal (cerebral cortex) regions. At a muscular level, morphologic changes will manifest as gross weakness or atrophy, ${ }^{1}$ which is problematic due to the high potential for altered 
biomechanics leading to excessive loading transmitted through lower extremity joints. ${ }^{20}$ At the spinal region, changes in reflex excitability of lower extremity musculature have been identified in patients with joint injury. ${ }^{21}$ Currently, the best way to measure central nervous system changes originating from supraspinal centers is to assess corticospinal excitability, which is defined as excitability of the portion of the cerebral cortex responsible for initiating motor commands to skeletal muscle. ${ }^{22}$ Transcranial magnetic stimulation (TMS) provides a method of assessing excitability of the pre-motor area of the cerebral cortex. ${ }^{22}$ TMS produces a small, but powerful field of magnetic energy that depolarizes neural tissue to initiate action potentials. ${ }^{22}$ When a TMS device is placed over the scalp, superficial to the pre-motor area, action potentials are conveyed to the associated skeletal muscles resulting in a motor evoked potential (MEP). Although neuromuscular adaptations are inevitable following joint injury, they present a modifiable source of dysfunction in the prevention of post-traumatic knee joint osteoarthritis.

Despite evidence suggesting that the central nervous system is significantly involved in mediating neuromuscular function following joint injury, there is a paucity of literature concerning intervention strategies directly targeting supraspinal centers. The corticospinal tract is the major descending pathway from the motor cortex to $\alpha$ - and $\gamma$-motor neurons, ${ }^{23}$ making this an important construct to study in response to peripheral injury. Theoretically, a Gaussian shaped curve of corticospinal excitability would occur over the course of recovery following injury. However, in cases of incomplete sensorimotor recovery, corticospinal excitability may not return to pre-injury levels, resulting in a reduction of clinical outcomes. A point of interest to clinicians is the theoretical high-risk zone, where individuals with good and poor outcomes diverge, indicating a critical junction in a targeted rehabilitation process (Figure A1). The figure below depicts theoretical changes that occur at supraspinal centers over time following knee joint trauma. 
To date, there is limited evidence with respect to specific neuromuscular adaptations within the central nervous system following ACL reconstruction $(\mathrm{ACL}-\mathrm{R}),{ }^{24}$ and no information regarding the temporal relationship of these factors following ACL injury through the development of post-traumatic

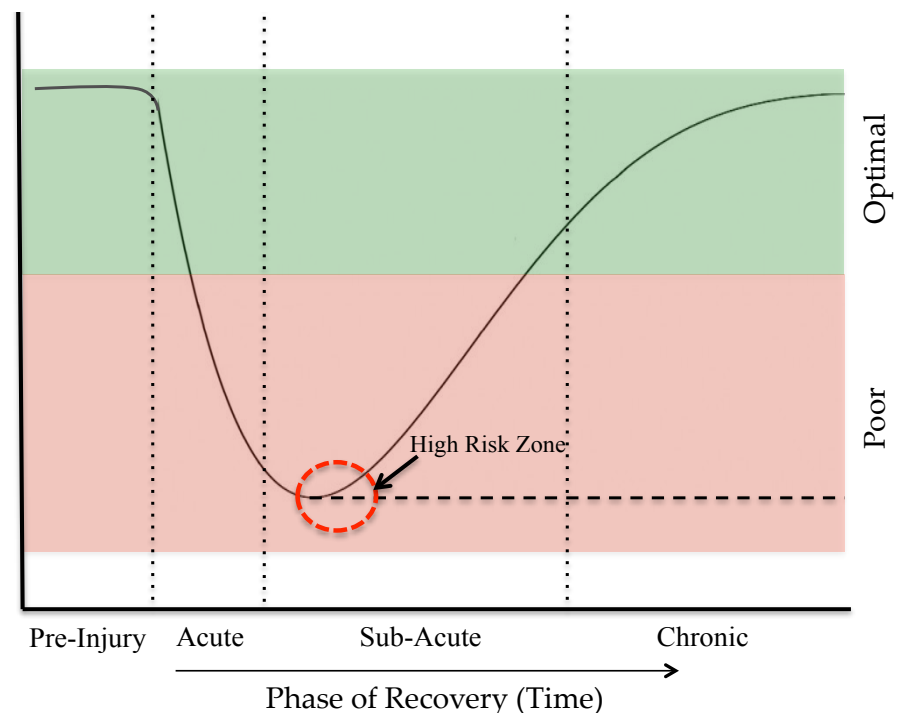

Figure A1. Theoretical model depicting the temporal relationship between quadriceps function and clinical outcomes following ACL reconstruction

joint degeneration. Current models of study have classified ACL-R patients as a single group of comparison relative to healthy counterparts (Figure A2). Exclusion of multiple time groupings may prevent early detection of impairments, delaying early intervention. In an effort to better understand how early state changes manifest over time, it is imperative that time from injury be considered in study design. Additionally, the inclusion of an end-stage model of disability is lacking in this regard.

In an effort to

effectively combat the detrimental plastic changes that may occur if left untreated, it is imperative for clinicians to identify impairments early in

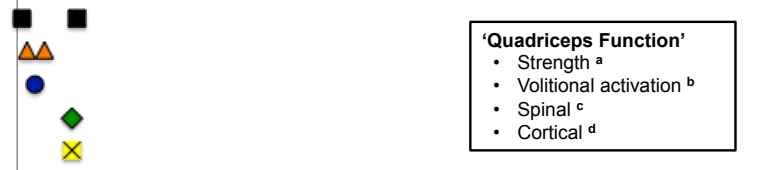
$x$
$+x-1$
+1

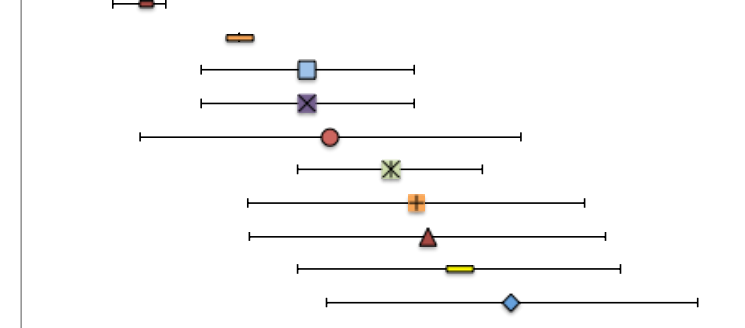

- Lepley et al. Scan J Med Sci Sport, 2015 a,b,c, d $\Delta$ Drechsler et al. Eur J Appl Physiol, 2006 a,b - Snyder-Mackler et al. JBJS, 1994 a,b $\diamond$ Chang et al. Med Sci Sports Exerc, 2014 a,b

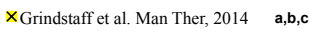
\$ Thomas A et al. J Sci Med Sport, 2015 a,b \#Lepley et al. KSSTA, 2014 a,b -Pfeifer et al. Scan J Med Sci Sports, 1999 a,b - Urbach et al. J Bone Joint Surg, 2001 a,b 口Kuenze et al. J Athl Train, $2015 \quad \mathbf{a}, \mathbf{b}, \mathbf{c}, \mathbf{d}$ × Kuenze et al. J Sport Rehabil, 2015 a,b - Hart et al. J Athl Train, $2014 \quad$ a,b,c * Davis et al. J Athl Train, $2011 \quad \mathbf{a}, \mathbf{b}$ \# Kuenze et al. J Athl Train, $2014 \quad$ a,b $\Delta$ Kuenze et al. J Sport Rehabil, $2013 \quad$ a,b - Lepley et al. Knee, $2014 \quad$ a,b,c,d $\diamond$ Pietrosimone et al. J Sport Rehabil, $2013 \quad$ a,d $\begin{array}{lllllllllllllllll}0 & 5 & 10 & 15 & 20 & 25 & 30 & 35 & 40 & 45 & 50 & 55 & 60 & 65 & 70 & 75 & 80\end{array}$ Months from Surgery (error bars $=$ SD)

Figure A2. Summary of studies that include a minimum of one measure of quadriceps neuromuscular function in addition to muscle strength in patients after ACL reconstruction. Point estimates indicate mean time from surgery, with error bars indicating standard deviation or range of time since surgery.

this continuum 
(Figure A3). Furthermore, it becomes necessary to identify characteristics of patients with poor clinical outcomes (e.g. osteoarthritis) to explore the relevance of early sensorimotor changes.

Therefore, the current study will use a cross-sectional design to compare differences in sensorimotor function among patients following ACL reconstruction at various phases of recovery through the development of post-traumatic joint degeneration.

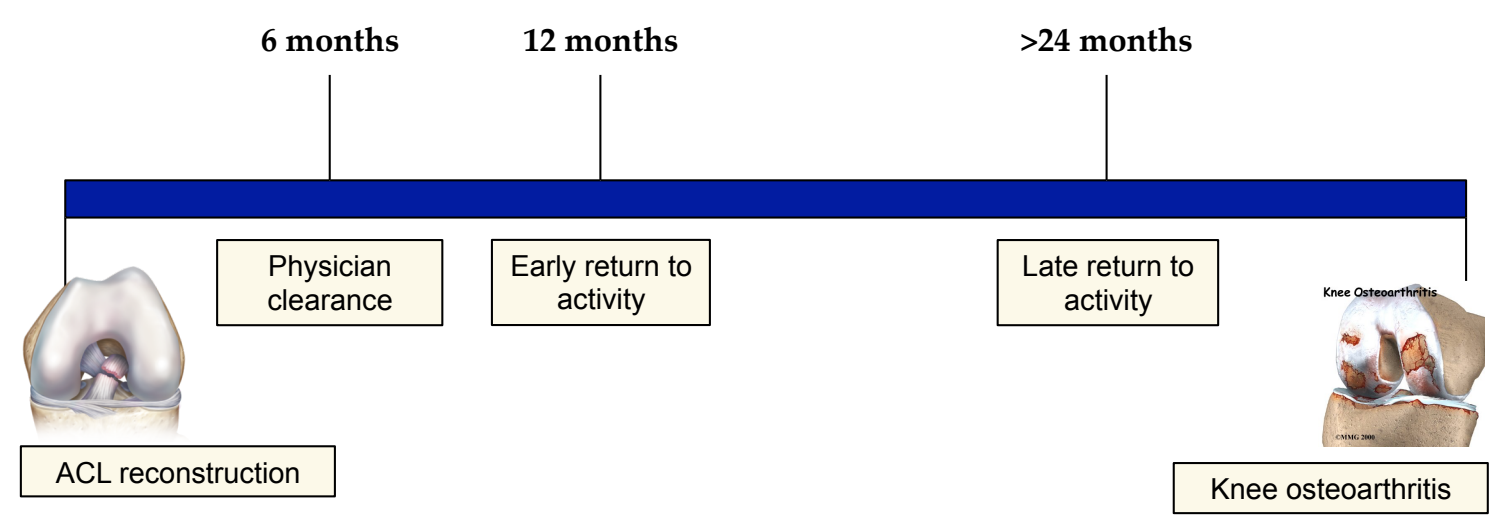

Figure A3. Represents the potential continuum of ACL reconstruction through the development of cartilage degeneration (sub-optimal outcomes) 


\section{Specific Aims}

The overall purpose of this study was to describe quadriceps muscle function over the course of recovery following ACL reconstruction (ACL-R) through development of posttraumatic osteoarthritis. This study included three specific aims to achieve this purpose. The primary aim of this study was to assess quadriceps neuromuscular function in patients after ACL$\mathrm{R}$ with regard to chronicity (early: 6-12 months, late: 2-10 years, and in those who experience post-traumatic knee osteoarthritis). Quadriceps function was assessed bilaterally via isometric and isokinetic knee extensor strength, quadriceps fatigue, spinal reflex excitability using the Hoffmann reflex, and corticospinal excitability using TMS to evaluate multiple regions of the central and peripheral nervous system. The purpose of this aim was to identify unique neurophysiological factors that distinguish ACL reconstructed patients with regard to time from surgery. The secondary aim of this study was to compare the relationships between measures of quadriceps function and patient reported function at clinically relevant phases after ACL-R. This aim provided information regarding the associations of unilateral and limb symmetry estimates of performance with perceived knee function and global health in patients early, late, and with knee osteoarthritis after ACL-R. This data will be used to assess the predictive capabilities of quadriceps function in determining clinical outcomes. The tertiary aim of this study was to identify unique constructs, or subgroupings, of muscle function that best characterize this patient population via principal component analysis. Within each construct, or component, relationships between individual variables will be assessed, and redundancy among measures will be identified, eliminating those that do not contribute unique information within this patient population. This analysis will identify important constructs of muscle function and maximize the efficiency of the assessment model in patients after ACL-R. 


\section{Research Question(s) and Experimental Hypotheses}

Manuscript 1: Chronicity of Quadriceps Function in ACL Reconstructed Patients With and

Without Knee Osteoarthritis

Research Question

Do peripheral (strength/fatigue/activation), spinal (spinal-reflexive excitability), and supraspinal (corticospinal excitability) measures of quadriceps function differ at clinical relevant phases (early: 6-12 months, late: 2-10 years, knee osteoarthritis) of postoperative recovery between ACL reconstructed patients with and without knee osteoarthritis and healthy matched controls?

Research Hypothesis

- Quadriceps strength, fatigue, activation, spinal-reflexive excitability, and corticospinal excitability will be worse in patients early after ACL-R, and in those with knee osteoarthritis, and will return to healthy values in patents late after ACL-R with no osteoarthritis.

\section{Statistical Analysis}

Separate mixed model $2 \times 4$ (limb x group) analyses of variance will be used to assess differences between the involved and uninvolved limbs for each measure of quadriceps function across all groups. Dunnett's post hoc tests will be used to identify the exact location of group differences between ACL-R patients and healthy controls if main effects are observed. Fischer's LSD post hoc tests will be used to identify group differences between ACL-R patients only. Separate models will be performed for the subjective data and the objective data (injured side vs. matched healthy control side; contralateral side vs. matched healthy control side; injured side vs. uninjured side). Finally as an exploratory analysis, side-to-side ratios (involved/uninvolved) will be calculated for an additional analysis to determine group differences in data when normalized within subject. Cohen's $d$ effect sizes with $95 \%$ confidence intervals will be 
calculated to assess the magnitude of difference between ACL-R patients and healthy controls. Alpha will be set at $\mathrm{p} \leq .05$, with $1-\beta=0.80$.

Manuscript 2: Relationship between quadriceps function symmetry and patient-reported outcomes in ACL reconstructed individuals with and without knee osteoarthritis

\section{Research Question 1}

Does unilateral quadriceps function or limb symmetry better explain patient reported outcomes early and late after ACL reconstruction in patients with and without knee osteoarthritis?

\section{Research Hypothesis 1}

- Limb symmetry will be most meaningful in patients early after ACL reconstruction

- Unilateral quadriceps function will be most meaningful in patients late after ACL reconstruction with and without osteoarthritis.

Research Question 2

Which measures of quadriceps function explain the greatest portion of variance in patient-reported function in patients early, late, and with osteoarthritis after ACL reconstruction?

Research Hypothesis 2

- Isokinetic strength (peak torque, total work, and average power) will explain the greatest variance in patient-reported function in each group.

\section{Statistical Analysis}

Simple bivariate correlations will be used to identify relationships among objective measures of quadriceps function and patient-reported outcomes. These coefficients will be calculated in separate analyses per group. We will perform a multiple linear stepwise regression analysis to identify the variance explained in patient-reported outcomes specific to knee (KOOS) and global health (VR-12) using (1) quadriceps 
function: isokinetic knee extensor strength (peak torque, total work, average power), knee extension MVIC torque, quadriceps fatigue index, quadriceps central activation ratio, Hoffmann reflex, active motor threshold, and (2) demographics: current patient age, time since surgery, pain, current activity level, and kinesiophobia.

Manuscript 3: Quadriceps and Patient Reported Function in ACL Reconstructed Patients: A Principal Component Analysis

Research Question

Which groupings of variables, or underlying constructs, representative of quadriceps, can be used to uniquely characterize ACL reconstructed patients?

Research Hypothesis

- Constructs related to (1) peripheral muscle function: quadriceps isokinetic strength, MVIC torque, fatigue index and (2) central muscle function: quadriceps central activation ratio, spinal-reflexive excitability, corticospinal excitability, will uniquely identify four distinct factors.

Statistical Analysis

A principal component analysis (PCA) will be performed using all dependent variables separately for the involved limb, uninvolved limb, and limb symmetry indices among ACL-R patients only. Simple bivariate correlations will be used to determine the relationship between variables that load onto like components to identify those that provide unique information with regard to this patient population. Receiver operator characteristic curve analyses will be used to establish thresholds for retained variables to discriminate between patients with and without ACL reconstruction. Finally, retained variables will be entered into a binary logistic regression model for the involved limb, uninvolved limb, and limb symmetry values to determine the ability to predict group membership. 


\section{Independent Variables}

Group

1. Early ACL reconstruction (6-12 months)

2. Late ACL reconstruction (2-10 years)

3. Osteoarthritis after ACL reconstruction

4. Healthy controls

Limb

1. Involved

2. Uninvolved

\section{Dependent Variables}

Patient Reported Outcomes

1. International Knee Documentation Committee (IKDC) Subjective Knee Joint Evaluation

2. Knee Injury and Osteoarthritis Outcome Score (KOOS)

3. Pain and Activity Rating Scale (VAS)

4. Tegner Activity Scale

5. Godin Leisure-Time Questionnaire

6. Tampa Scale of Kinesiophobia (TSK)

7. Veterans Rand 12-Item Health Survey (VR-12)

8. Marx Activity Scale

Peripheral Muscle Function

1. Normalized isokinetic knee extension peak torque $(\mathrm{Nm} / \mathrm{kg})$, total work $(\mathrm{J} / \mathrm{kg})$, and average power $(\mathrm{W} / \mathrm{kg}) @ 90 \%$ second and $180^{\circ} /$ second

2. Normalized knee extension maximal voluntary isometric contraction torque $(\mathrm{Nm} / \mathrm{kg})$

3. Quadriceps fatigue index $(\%)$

Central Muscle Function

1. Quadriceps central activation ratio (\%) 
2. Normalized quadriceps Hoffmann reflex (H:M ratio)

3. Quadriceps active motor threshold (\% MSO)

\section{Inclusion Criteria}

Healthy Controls

- 15-65 years old

- No prior knee injury

- No recent hip or ankle injury (last 6 weeks)

- $\quad$ BMI less than 35

- No history or immediate family history of seizures or epilepsy

ACL Reconstruction

- 15-65 years old

- No recent hip or ankle injury (last 6 weeks)

- History of ACL injury and/or reconstruction

- $\quad$ BMI less than 35

- No history or immediate family history of seizures or epilepsy

ACL Reconstruction with Osteoarthritis

- 15-65 years old

- Diagnosed tibiofemoral or patellofemoral osteoarthritis (Kellgren-Lawrence grade 2-4)

- $\quad$ No recent hip, knee, or ankle injury (last 6 weeks)

- History of ACL reconstruction (revision accepted)

- $\quad$ BMI less than 35

- No history or immediate family history of seizures or epilepsy

\section{Exclusion Criteria}

Healthy Controls

- Currently experiencing knee pain 
- History of knee joint injury or surgery

- Current neuropathy (numbness and tingling)

- Known muscular abnormality

- History of skull fracture

- History of neurological disorders including poorly controlled migraine headaches, seizure disorder, history or immediate family history of seizures and/or epilepsy and taking medications that lower seizure threshold

- History of subdural hematoma or epidural hematoma

- Implanted biomedical device (active or inactive implants (including device leads), including deep brain stimulators, cochlear implants, and vagus nerve stimulators)

- Conductive, ferromagnetic or other magnetic-sensitive metals implanted in their head or within $30 \mathrm{~cm}$ of the treatment coil. Examples include cochlear implants, implanted electrodes/stimulators, aneurysm clips or coils, stents, bullet fragments, jewelry and hair barrettes.

- History of cardiopulmonary disorder

- Pregnant women

- Significant activity change 48 hours prior to enrollment

ACL Reconstruction

- Multiple ligament reconstruction or a history of graft failure

- Serious surgical complication following ACL reconstruction

- Chondral resurfacing procedure (microfracture or OATS procedure)

- History of cardiopulmonary disorder

- Current symptoms of meniscal injury or failed meniscal repair

- Current neuropathy (numbness and tingling)

- Known muscular abnormality 
- History of skull fracture

- History of neurological disorders including poorly controlled migraine headaches, seizure disorder, history or immediate family history of seizures and/or epilepsy

- Taking medications that lower seizure threshold

- History of subdural hematoma or epidural hematoma

- History of neurological disorders

- Implanted biomedical device (active or inactive implants (including device leads), including deep brain stimulators, cochlear implants, and vagus nerve stimulators)

- Conductive, ferromagnetic or other magnetic-sensitive metals implanted in their head or within $30 \mathrm{~cm}$ of the treatment coil. Examples include cochlear implants, implanted electrodes/stimulators, aneurysm clips or coils, stents, bullet fragments, jewelry and hair barrettes

- Pregnant women

- Significant activity change 48 hours prior to enrollment

ACL Reconstruction with Osteoarthritis

- Diagnosis of osteoarthritis prior to ACL reconstruction

- Less than 1 year from ACL reconstruction

- Prior knee replacement (partial or total)

- Knee surgery on the contralateral limb

- Multiple ligament reconstruction

- Chondral resurfacing procedure (microfracture or OATS procedure)

- Recent knee surgery within 6 months

- History of cardiopulmonary disorder

- Current neuropathy (numbness and tingling)

- Known muscular abnormality 
- History of skull fracture

- History of neurological disorders including poorly controlled migraine headaches, seizure disorder, history or immediate family history of seizures and/or epilepsy

- Taking medications that lower seizure threshold

- History of subdural hematoma or epidural hematoma

- History of neurological disorders

- Implanted biomedical device (active or inactive implants (including device leads), including deep brain stimulators, cochlear implants, and vagus nerve stimulators)

- Conductive, ferromagnetic or other magnetic-sensitive metals implanted in their head or within $30 \mathrm{~cm}$ of the treatment coil. Examples include cochlear implants, implanted electrodes/stimulators, aneurysm clips or coils, stents, bullet fragments, jewelry and hair barrettes

- Pregnant women

- Significant activity change 48 hours prior to enrollment

\section{Procedures}

1. Obtained informed consent

2. Screening (TMS questionnaire)

3. Patient-reported function questionnaires

a. International Knee Documentation Committee Subjective Knee Evaluation

b. Knee Injury and Osteoarthritis Outcome Score

c. Pain and Activity Rating Scale

d. Tegner Activity Scale

e. Godin Leisure-Time Exercise Questionnaire

f. Tampa Scale of Kinesiophobia

g. Veterans Rand 12-Item Health Survey 
4. Bilateral Hoffmann reflex

5. Bilateral quadriceps isokinetic strength

6. Bilateral quadriceps central activation ratio

7. Bilateral quadriceps fatigue index

8. Bilateral quadriceps active motor threshold

\section{Assumptions}

- The obtained measures are valid and reliable

- Participants responded truthfully to questionnaires

- Participants provided maximal effort during strength assessment(s)

- The quadriceps central activation ratio (CAR) was representative of force generated by activated motor units recruited volitionally when compared to the maximal capacity of the muscle

- The transcutaneous electrical stimulation utilized during the quadriceps superimposed burst technique was sufficient to activate all quadriceps muscle tissue not active during a maximal voluntary isometric contraction

- Participants were fully relaxed during quadriceps Hoffmann reflex testing.

- Surface electromyography adequately represented the true activation of the quadriceps muscle during active contractions

- The quadriceps Hoffmann reflex was representative of quadriceps motorneuron pool excitability

- The area of the pre-motor cortex stimulated during quadriceps active motor threshold testing was the optimal location for stimulation resulting in the largest magnitude response in the target muscle

- Participants were assessed in a non-fatigued state

- Participants did not have caffeine within 6 hours prior to being assessed 
- Participants were not taking medications that could alter spinal or corticospinal excitability

- Participants with no diagnosis of knee osteoarthritis truly did not have arthritic changes at the time of enrollment

- Participants with diagnosed knee osteoarthritis did not have arthritic changes prior to ACL-R

\section{Delimitations}

- Physically active individuals between the ages of 15-65 years

- Primary, unilateral and uncomplicated ACL reconstruction

- Time from ACL reconstruction: (1) 6-12 months, (2) 2-10 years, (3) diagnosed osteoarthritis after ACL-R

- Knee osteoarthritis (Kellgren Lawrence $\geq$ grade 2) with a history of unilateral ACL reconstruction

\section{Limitations}

- Cross-sectional design does not allow for conclusions regarding the natural history of post-traumatic neuromuscular function of the quadriceps

- Healthy controls were not age matched to the osteoarthritis patient group

- Unable to confirm severity of arthritis at time of enrollment in patients with arthritis

- Unable to verify absence of arthritis in patients with no diagnosis of osteoarthritis

- Heterogeneous sample with regard to age, gender, graft type, and meniscal involvement

- Principal component analysis may overestimate the variance explained among measures of quadriceps neuromuscular function 


\section{Operational Definitions}

1. Active motor threshold - The TMS intensity that produces a positive MEP measured locally at the target muscle in at least 5 out of 10 trials during an active contraction, measuring $5 \%$ of maximal effort. ${ }^{25}$

2. Arthrogenic muscle inhibition - Presynaptic, ongoing reflex inhibition of musculature surrounding a joint after distension or damage to structures of that joint. ${ }^{26}$

3. Cortical plasticity - The capacity of the nervous system to modify its organization. Such changes can occur as a consequence of many events, including normal development, the (re)acquisition of skills (learning/ relearning), after damage to the nervous system and as a result of sensory deprivation. ${ }^{27}$

4. Corticospinal excitability - The ability of corticospinal neurons within the primary motor cortex to be activated in response to input from sensory centers, the pre-motor cortex, the spinal cord, and the basal ganglia. ${ }^{28}$ The intensity of input signal require to evoke excitation of the area related to the quadriceps in the primary motor cortex is commonly measured using the active motor threshold via transcranial magnetic stimulation. ${ }^{29}$

5. Chronicity - Refers to time from injury/ surgery or progression of condition.

6. H:M ratio - A representation of the motorneuron pool available to be recruited $\left(\mathrm{H}_{\max }\right)$ compared to the entire motorneuron pool $\left(\mathrm{M}_{\max }\right)$. The ratio can be interpreted as the proportion of the motorneuron pool available for recruitment at a given time. ${ }^{30}$

7. Hoffmann reflex (H-reflex) - An electrically induced reflex analogous to the mechanically induced spinal stretch reflex, ${ }^{30}$ which provides an estimate of alpha motoneuron excitability when presynaptic and intrinsic excitability remain constant. The H-reflex is elicited via direct electrical stimulation of the peripheral nerve that results in preferential depolarization of Ia afferent fibers at low stimulus intensities. The maximum H-reflex (Hmax) represents the maximum number of motorneurons that can be activated in a given state of condition. 
8. Limb symmetry - The comparison of within participant, between-limb performance on a given outcome measure that is represented as a unit-less ratio. This limb symmetry index (LSI) is represented as: ACL-R LSI = Injured Limb/ Uninjured Limb or Healthy LSI = Non-Dominant Limb/ Dominant Limb.

9. Motor evoked potential - Artificial depolarization of cortical neurons via electromagnetic stimulation over the motor cortex.

10. Motorneuron pool excitability - The percentage of the total motorneuron pool that can be achieved at rest or during active contraction in response to an applied stimulus, which is commonly estimated using the Hoffmann reflex and expressed as the $\mathrm{H}_{\max }: \mathrm{M}_{\max }$ ratio. ${ }^{30}$

11. Motor threshold - The TMS intensity that produces a positive MEP measured locally at the target muscle in at least 5 out of 10 trials. $^{25}$

12. Muscle response (M response) - The efferent arc of the H-reflex pathway, resulting from action potentials generated by the alpha motoneurons traveling along efferent fibers, until they reach the neuromuscular junction and produce a twitch response in the electromyograph. ${ }^{30}$ The maximal $\mathrm{M}$ response $\left(\mathrm{M}_{\max }\right)$ represents activation of the entire motorneuron pool, and is commonly used as a normalization factor for the maximal Hoffmann reflex $\left(\mathrm{H}_{\max }\right) .^{31}$

13. Neuromuscular Control - Any of the aspects surrounding nervous system control over muscle activation, and the factors contributing to task performance. The unconscious activation of dynamic restraints occurring in preparation for and in response to joint motion and loading for the purpose of maintaining and restoring functional joint stability. $^{23}$

14. Post-traumatic osteoarthritis - Diagnosed Kellgren-Lawrence Grade 2 or higher tibiofemoral osteoarthritis with possible patellofemoral compartment involvement, secondary to joint trauma. 
15. Proprioception - The afferent information arising from 'proprioceptors' located in the 'proprioceptive field', the area of the body screened from the environment by the surface cells, which contained receptors specifically adapted for the changes occurring inside the organism independent of the interoceptive field. ${ }^{32}$

16. Quadriceps activation $(\boldsymbol{Q A})$ - The proportion of motor neuron pool that can be volitionally activated during a force-based task. ${ }^{1}$

17. Quadriceps central activation ratio $(\boldsymbol{C A R})$ - A ratio of the maximal voluntary isometric force $\left(\mathrm{F}_{\mathrm{MVIC}}\right)$ to the total force generated when a supramaximal percutaneous electrical stimulus is superimposed during a maximal voluntary isometric contraction $\left(\mathrm{F}_{\mathrm{SIB}}\right){ }^{1}$ commonly expressed as: $\mathrm{CAR}=\left[\mathrm{F}_{\mathrm{MVIC}} /\left(\mathrm{F}_{\mathrm{MVIC}}+\mathrm{F}_{\mathrm{SIB}}\right)\right]$ a 100 . A CAR of 1.0 indicates complete activation, whereas a CAR of less than 1.0 indicates central activation failure or inhibition. $^{33}$

18. Quadriceps inhibition (QI) - A reduction in central motor drive to the quadriceps musculature, which results in a decreased ability to generate maximal volitional activation of the muscle, commonly expressed as: $\mathrm{QI}=1-\mathrm{CAR} .^{33}$

19. Sensorimotor Control - The dynamic interaction between sensation of sensory information, the integrating of information in the central nervous system and motor output to perform voluntary movements and postural control. ${ }^{34}$

20. Sensorimotor System - The sensory, motor, and central integration and processing components involved in maintaining joint homeostasis during bodily movements (functional joint stability). ${ }^{35}$

21. Somatosensory - All mechanoreceptive, thermoreceptive, and pain information arising from the periphery. ${ }^{36}$

22. Spinal reflexive excitability - Refers to the magnitude of alpha motoneuron excitability when presynaptic and intrinsic excitability remain constant. This will be synonymous to H:M ratio. 
23. Superimposed burst technique (SIB) - Application of a train of percutaneous supramaximal electrical stimuli to the quadriceps musculature during a maintained voluntary contraction, ${ }^{37}$ used to quantify the extent of voluntary activation failure of a muscle. ${ }^{38}$

24. Transcranial magnetic stimulation - A method for studying the relationship between brain activity and physical function through the use of electromagnetic stimulation of the motor cortex to generate a motor evoked potential which can be measured over the targeted muscle via electromyography. ${ }^{29}$

25. Voluntary activation failure - The inability to produce all available force of a muscle despite maximal conscious effort. ${ }^{39}$ 


\section{Innovation}

Athletic trainers are situated as primary health care providers able to identify early risk factors for poor outcomes following joint injuries, such as ACL tears and reconstruction. The process of joint degeneration following trauma is irreversible, making it imperative that clinicians and researchers alike continue to make strides in optimizing patient care following injury.

Despite evidence suggesting that the central nervous system is significantly involved in mediating neuromuscular function following joint injury, there is a paucity of literature concerning intervention strategies directly targeting supraspinal centers. The lack of such studies raises the question: are current rehabilitative efforts comprehensive enough to address the neuromuscular impairments observed in response to ACL injury and reconstruction? How is peripheral, spinal, and supraspinal input modulated over time following joint injuries? To optimize patient care, these questions must be addressed. Understanding the natural history of neuromuscular modulation from peripheral, spinal, and supraspinal centers is a necessary step in answering this gap of knowledge. Examining the inter-relationships of these sources of neuromuscular modulation from a temporal perspective is paramount in developing evidence based treatment strategies. In a continuing shift towards evidence-based practice, researchers and clinicians must gain a more comprehensive understanding of underlying pathophysiologic mechanisms of neuromuscular adaptations following knee joint injuries. Gaining insight with regard to the neuromuscular adaptations that occur throughout the spectrum of disability, from injury to development of degenerative changes, will provide information paramount to develop optimal strategies for early treatment and active prevention of poor outcomes in active individuals who suffer joint injuries. Understanding the temporal relationship of neural mechanisms involved in mediating neuromuscular function following a common knee joint injury is a necessary step to validate patient-specific interventions. Central reorganization is a naturally occurring phenomenon following injury; therefore, evaluating supraspinal sources of neuromuscular impairment across a spectrum of disability will provide insight into the naturally occurring 
adaptive responses that take place following injury, specifically plastic changes within the primary motor cortex. It is imperative that clinicians identify impairments along the continuum of injury and disability to combat unwanted plastic changes, detrimental to long-term joint health. Exploring the lesser understood role of supraspinal excitability and centrally mediated changes over time will help clinicians understand how to most effectively treat patients, and may provide a missing link in current practice.

Long-term benefits to the proposed study have both clinical and research implications. Clinically, information gained from this study can be used to understand how different patient populations adapt following injury from a neurophysiologic perspective. Clinicians must understand how individuals adapt over time to truly understand and predict the effects of therapeutic interventions. Pairing the proposed neurophysiologic observations with patient reported outcomes would establish the connection between neuromuscular adaptations and quality of life following injury. Having these data may allow clinicians to predict outcomes, and intervene at earlier points in order to avoid permanent detrimental changes in neuromuscular function. This information will provide a crucial dataset for identifying early changes following ACL injury. The data from this study will be used for future, larger scale applications to study how these regions mediate neuromuscular function over time following various knee injuries. Although the proposed study utilizes a cross-sectional design, it will be used to provide insight moving forward with longitudinal observations. 


\section{APPENDIX B}

\section{Literature Review}

\section{INTRODUCTION}

Knee joint injuries remain common among athletic and recreationally active populations. Unfortunately, early mal-adaptations throughout the central nervous system can occur following injury, resulting in long-term consequences with regard to joint health and decreased quality of life. Anterior cruciate ligament (ACL) injuries present a specific challenge to joint health in this regard and continue to constitute a large portion of major knee joint injuries in young, active populations. Post-traumatic muscle dysfunction is well described following ACL reconstruction, ${ }^{1}$ and is linked to a sequelae of impairments detrimental to global and joint-specific health, including decreased physical activity, ${ }^{2-5}$ increased risk of re-injury, ${ }^{6}$ and an accelerated onset of post-traumatic knee joint osteoarthritis. ${ }^{7-11}$ Strong evidence exists supporting the causal link between ACL-R and early post-traumatic articular cartilage joint degeneration. ${ }^{9,12-14}$ Since articular cartilage degeneration is irreversible, the hallmark for prevention is early detection. Neuromuscular adaptations are inevitable following joint injury, but present a modifiable source of dysfunction in the prevention of post-traumatic knee joint osteoarthritis. To date, there is limited evidence with respect to changes within the central nervous system following ACL reconstruction (ACL-R), ${ }^{24}$ and no information regarding the temporal relationship of these changes following ACL injury through the development of post-traumatic joint degeneration. 


\section{EPIDEMIOLOGY OF ANTERIOR CRUCIATE LIGAMENT INJURY}

Epidemiologic data regarding the incidence of ACL injury and reconstruction has been widely studied, however, remains considerably variable, as it is often based on expert opinion and limited electronic databases. Several studies have examined trends over time in the ACL injury. In 2007, Hootman et al. ${ }^{40}$ reported a 1.3\% annual increase in ACL injuries from 1988-2004 using data from the NCAA Injury Surveillance System collected from 15 sports. Approximately 5,000 ACL injuries were reported to this system during this time, with an average annual occurrence of 313. ACL rupture remains common in sports, ${ }^{41}$ and reconstruction is often recommended to facilitate return to sport. ${ }^{42}$ In the United States, the incidence of ACL reconstruction (ACLR) was reported to rise from 86,687 (32.9 per 100,1000 person years) in 1994 to 129,836 (43.5 per 100,000 person years) in $2006 .{ }^{43}$ Varying incident is reported, however, similar trends in rate of reconstruction have been reported in the United States. In 2009, Lyman et al ${ }^{44}$ reported a $22 \%$ increase in ACLR from 1997 to 2006 using data from hospital admissions in New York State. The decision to undergo ligament reconstruction following ACL rupture is multifactorial and patient specific. Within an active population, ACLR is often selected in an attempt to prevent further injury.

\section{RISK FOR INJURY}

A multitude of factors, both internal and external, may contribute to risk of ACL injury. Within an active population, ACL ruptures remain one of the most common knee injuries. ${ }^{45}$

\section{$\operatorname{Sex}$}

The relationship between patient sex and risk for ACL injury has been widely studied in a sports medicine context. Data is somewhat conflicting regarding the number of ACL injuries sustained by male and female counterparts. Higher incidence rates for ACL injury has been

identified in males relative to female counterparts using data from a population-based study. ${ }^{46}$ However, when comparing rates of injury within sport, a 2007 meta-analysis revealed an increased occurrence among female athletes, citing a 3 times greater incidence in soccer and 
basketball specifically. ${ }^{47}$ A variety of suggested risk factors including environmental factors, anatomical indices, hormonal influences, and biomechanical factors have been reported to contribute to ACL injuries in females. ${ }^{48-50}$

Age

The number of ACL reconstructions in young, active populations has continued to increase over the past several decades. ${ }^{44}$ In patients younger than 20 years, the average number of ACLRs increased from 12.2 to 18.0 per 100,000 person-years between 1996 and 2006.

Additionally, this age group comprised a greater proportion of all ACL injuries compared to patients in their $2^{\text {nd }}, 3^{\text {rd }}$, or $4^{\text {th }}$ decade of life..$^{43}$ Although the cause of this increase is unknown, it is possible that an increase in sport participation or duration of athletic activities throughout the year may contribute to this finding.

Sport

Epidemiologic studies using large electronic data capture systems have provided valuable insight on injury rates by sport. In collegiate athletics, the NCAA Injury Surveillance System (ISS) has been used to obtain these data. Data from 15 sports were obtained between 1988 and 2004. ACL injury rates have been reported as highest in football, women's gymnastics, women's soccer, and women's basketball. ${ }^{40}$ These data support evidence of increased risk for ACL injury in soccer and basketball, specifically among female athletes. Because these sports mimic the cutting tasks associated with ACL injury, research among such athletes has been a specific interest in sports medicine. Prodromos et al. $2007^{47}$ reported combined injury rates for female soccer and basketball players at 0.3 per 1,000 exposures, which they equate to an approximate $5 \%$ annual risk for injury. Similarly, previous data has reported a 4.4\% 1-year incidence of ACL injury in this population. ${ }^{51}$

\section{RISK FOR RE-INJURY AND POST-TRAUMATIC KNEE OSTEOARHTRITIS}

Mitigating the risk for re-injury is a primary concern for health care practitioners when making rehabilitative and return to activity decisions. The greatest predictor for future injury is a 
history of previous injury, and unfortunately, not all risk factors for secondary injury can be modified. It is however important for clinicians to be aware of risk factors, internal and external, that may be considered by varying members of the sports medicine team.

\section{Early (ACL Reconstruction - Return to Play at 2-15 Years)}

\section{Graft Type}

Many studies have compared short and long-term outcomes among graft types, most notably among patellar tendon and hamstring tendon grafts. In 2011, a systematic review ${ }^{52}$ examined clinical outcomes in ACL deficient patients with patellar tendon and hamstring autografts. From nineteen studies reporting follow up data 2-8.5 years post ACLR, the authors of this review concluded that there is insufficient evidence to support the clinical benefit of either graft choice. In partial support of this finding, a recent prospective analysis of patients 15 years following ACLR, no differences in incidence of further ACL injury were noted between those with patellar tendon (8\%) or hamstring tendon (17\%) autograft. ${ }^{53}$ Although graft type was not associated with graft injury, a 2.6 increase in odds of contralateral ACL rupture was observed among patients with patellar tendon (26\%) graft compared to hamstring (12\%). Additional consideration and debate has been given with regard to use of single- and double-bundle grafts. A recent comparative study of over 16,000 patients revealed no difference in rate of ACL revision between those with single and double-bundle hamstring autograft. ${ }^{54}$

\section{Gender, Age, Race, Ethnicity}

Gender influence on injury rates is a debated topic in musculoskeletal research. Much of the literature examining risk of primary ACL injury indicates women are at an increased risk for injury. For the same reasons, women remain susceptible to re-injury, and have been cited as having a higher rate of ACL graft rupture than men. ${ }^{55}$ Within an athletic context, female soccer players are more likely than men to sustain second ACL injury (20\% vs. $5.5 \%) .{ }^{56}$ Additionally, women have been reported to be at greater risk for contralateral ACL injury at 12 and 24 months following return of sport. ${ }^{57,58}$ In contrast, a recent prospective analysis of patients 15 years after 
ACLR, reported that men were 3.2 times more likely to sustain and ACL graft rupture than women. ${ }^{53}$ Although this conflicts with previous data, this finding may be a product of men returning to higher-level activities than female counterparts. These findings are corroborated by a prior systematic review, concluding insufficient evidence to determine superiority in graft bundle. $^{59}$

Age at time of injury and subsequent surgery has been identified as a factor related to risk of re-injury with conflicting evidence. Data from the Swedish national ACL register identified age at the time of surgery $\left(<16\right.$ years) as a significant predictor of ACL revision at 5 years. ${ }^{60} \mathrm{~A}$ retrospective analysis of high-level athletes ranging 16-53 years of age, did not find an association between age and re-injury rates. ${ }^{61}$ Although a significant association was not found, athletes under 18 years of age demonstrated a re-injury rate of $8.7 \%$, whereas, only $2.6 \%$ of those 18-25 years sustained a second injury. Interestingly, Leys et $\mathrm{al}^{53}$ reported that graft rupture was not associated with age less than 18; however, young individuals were 4.1 times more likely to sustain a contralateral injury.

Race and ethnicity have been linked with risk of ACL injury, although evidence is lacking. In a retrospective study ${ }^{62}$ of female athletes in the Women's National Basketball Association, White European Americans (WEA) were determined to be $6.55 \%$ more likely to sustain a primary ACL injury than non-White European American players (African American, Hispanic, Asian). Furthermore, this study noted that WEA injury rates were greater $(0.45$ per 1,000 exposures) compared to overall ACL injury rates in the WNBA ( 0.20 per 1,000 exposures). Although the cause is not clear, Shelbourne et $\mathrm{a}^{63}$ reported that the intercondylar notch width measured during flexed weight bearing radiographs were significantly wider among African Americans than White European American counterparts, which may partially explain the increased incidence of ACL injuries among non-White European Americans. 


\section{Time}

Incidence of a second ACL injury in the first 12 months following reconstruction in a young, active population has been reported as 15 times greater than a previously uninjured cohort, ${ }^{57}$ and 6 times greater at 24 months. ${ }^{58}$ Nearly $30 \%$ of athletes sustained a second ACL injury within 24 months of return to sport ( $\sim 21 \%$ contralateral, $9 \%$ ipsilateral). Secondary injury data within 24 months of reconstruction appear to highlight the importance of continued rehabilitative efforts following ACL injury. Timing is commonly used as a primary criterion in return to sport decision-making. A lack of association between time from surgery and persistent functional deficits has been identified in athletes after ACLR, ${ }^{64}$ supporting the inclusion of more objective criterion when making return to play decisions. A 2010 retrospective analysis of 298 patients four years post ACLR revealed that athletes returning to competition within seven months from surgery were at a greater risk of re-injury than those returning at a later time $(15.3 \%$ vs. $5.2 \%)^{61}$

\section{Late (Late Stage Return to Play - Osteoarthritis)}

\section{Concomitant Injury}

National data from the Swedish national ACL register reported 5-year post-operative rates of revision (4.3\%) and contralateral ACL reconstruction (3.8\%) among nearly 21,000 patients. ${ }^{60}$ This study further identified concomitant injury at the time of ACL rupture as a significant risk factor for secondary ACL reconstruction, including operative and non-operative meniscus, articular cartilage, and collateral ligament injuries. Of note, surgically treated meniscus injuries were identified in $78.8 \%$ and $79.1 \%$ of all patients who sustained a revision or contralateral ACL reconstruction respectively. Due to the high prevalence of meniscal injuries at the time of ACL rupture, early articular cartilage degeneration in this population is a concern. A 2009 systematic review ${ }^{65}$ aimed to identify the impact of meniscal injury at time of ACL reconstruction on the development of osteoarthritis. This review concluded that patients who underwent partial meniscectomy at the time of ACL reconstruction were at a significantly greater 
risk for developing radiographic evidence of osteoarthritis at 5-10 year follow up, whereas, inconsistent findings were reported for those who underwent meniscal repair.

\section{Muscle Function}

Persistent quadriceps weakness and central activation failure are widely reported following knee joint injury. ${ }^{1}$ Post-traumatic muscle dysfunction is linked to a sequelae of impairments detrimental to global and joint-specific health, including decreased physical activity, ${ }^{3,4}$ increased risk of re-injury, ${ }^{6}$ and an accelerated onset of knee joint osteoarthritis. ${ }^{10,66}$ Strong evidence exists supporting the causal link between ACL-R and early post-traumatic articular cartilage joint degeneration. ${ }^{9,12-14}$ Central and peripheral neural adaptations accompany these consequences, and are established as an underlying contributor to muscle impairment. ${ }^{19}$ Quadriceps dysfunction is reported to manifest via altered excitability from spinal and cortical regions. ${ }^{67,68}$ The diminished ability to activate otherwise healthy peri-articular muscular tissue in the presence of joint pathology is termed arthrogenic muscle inhibition (AMI), and is proposed as a neural phenomenon responsible for limiting the progression of rehabilitation. ${ }^{26}$ Following injury, this arthrogenic response can manifest as an ongoing reflex inhibition due to aberrant sensory information arising from mechanoreceptors located in peri-articular structures, which the central nervous system (CNS) interprets as inhibitory. ${ }^{26,69}$ Inhibition of surrounding musculature may therefore result from transmission of aberrant afferent stimuli, and has been examined following artificial joint effusion, ${ }^{70}$ pain, ${ }^{71}$ and structural damage. ${ }^{68}$

\section{Post-Traumatic Osteoarthritis}

ACL rupture and subsequent reconstruction have been linked with early onset of articular cartilage degeneration. Specifically, medial compartment and patellofemoral joint osteoarthritis (OA) have been identified in patients following ACLR. In a recent systematic review of ACLR individuals, the authors concluded that there is insufficient evidence to suggest that ACL is adequate to prevent knee OA. This review identified radiographic signs of OA in $44 \%$ of all ACLR patients $(n=2,500)$. The prevalence of OA varied by isolated ACL rupture $(42 \%)$ and 
presence of meniscus injury (52\%). When further stratified by study design, retrospective studies reported 39\% $(\mathrm{n}=1,455)$, whereas, prospective studies demonstrated a $56 \%$ occurrence of OA ( $\mathrm{n}$ $=1,099$ ) during $3.9-35$ years follow up. ${ }^{72}$ Although much attention has been given to the development of tibiofemoral OA in the medial compartment, the prevalence of patellofemoral joint OA has been reported to range 11-90\% (median 36\%) within 2-15 years from surgery. ${ }^{2,73}$

\section{CLINICAL OUTCOMES}

Clinical outcomes are comprised of impairment-based and patient-oriented factors, including joint stability, muscle function, activity level, and self-reported function.

\section{Short-Term (0-2 years)}

Return to pre-injury activity levels following ACL reconstruction is a common goal among active individuals. In an updated systematic review of 4,837 ACL reconstructed patients, $81 \%$ returned to some form of sporting activity, whereas, $65 \%$ returned to pre-injury activity levels, and only $55 \%$ returned to competitive levels of sport. ${ }^{74}$ These data highlight the difficulties in returning to high-level sporting activities following ACL-R, which presents a specific challenge to young, active patients pursuing high-level athletics. The inability to return to specific activities is likely multifactorial. Symmetry of hopping performance, and contextual factors of younger age, male gender, playing an elite sport, and having a positive psychological response increased the change of return to pre-injury levels of sport. ${ }^{75}$ Impairment-based knee function has yielded considerably positive results following ACL-R, making, the psychological response to injury a recent topic of study in this regard. A positive psychological response to measures of readiness to return to sports participation (ACL-Return to Sport after Injury Scale) and fear of reinjury (Tampa Scale of Kinesiophobia) prior to and early after ACL-R significantly classified return to pre-injury activity level in a cohort of 187 athletes. ${ }^{76}$

\section{Medium (2-10 years)-Long-Term (10+ years)}

In a cohort of 314 ACL reconstructed individuals at a mean of 39.6 months from surgery were surveyed to determine the proportion of people participating in pre-injury activity levels and 
competitive sport. ${ }^{77} 45 \%$ were identified as playing sport at pre-injury levels, and only $29 \%$ were playing competitive sport. Additionally, early return to activity at 12 months was not predictive of activity level at 39 months in this sample, suggesting that people who return early may not maintain sports participation. Interestingly, a lack of association between high satisfaction and increased activity levels has been observed following ACL-R. In a retrospective review of 29 skeletally immature patients, $41 \%$ returned to pre-injury activity levels, despite reporting a mean satisfaction score of 9 (range, 4-10) and Lysholm score of 91 (range, 61-100) at 2 years. ${ }^{78}$

\section{PROBLEMS PATIENTS ARE FACED WITH FOLLOWING ACL RECONSTRUCTION}

\section{Sources of Sensorimotor Dysfunction}

The resulting inhibition of surrounding musculature that occurs following ACL injury may has been theorized to result from transmission of aberrant afferent stimuli, and has been examined in the presence of artificial joint effusion ${ }^{70}$, inflammation ${ }^{79}$, pain $^{71}$, and/ or structural damage. Reduced quadriceps muscle function has been reported to manifest as altered excitability at the spinal and cortical level ${ }^{67,68}$, including reduced volitional activation ${ }^{1}$, torque ${ }^{80}$, and electromyographic activity ${ }^{81}$ among individuals with artificial and true knee joint pathology.

\section{Peripheral receptors}

The location of sensory nerves has been suggested to be of particular importance to clinicians when treating arthrogenic muscle inhibition following joint injury. ${ }^{26}$ Somatosensory information originating from peripheral receptors is reported to influence motor function via projections to spinal motoneurons, as well as supraspinal structures. ${ }^{82}$ Specifically, sensory nerves innervating knee joint terminating in specialized mechanoreceptors have been theorized to play a primary role in modulating inhibition. ${ }^{83}$ It has been established that articular mechanoreceptors play a significant role in regulating afferent signals to the central nervous system, and appear to be sensitive to change in the presence of joint damage,${ }^{84}$ making the neurophysiological response of these receptors a clinical interest. 


\section{Spinal}

Research to date has largely focused on spinal mechanisms of AMI. ${ }^{19}$ The Hoffmann reflex (H-reflex) is a common neurophysiologic test used in sports medicine research to assess modulation of monosynaptic reflex activity in the spinal cord..$^{30}$ The H-reflex can be viewed as analogous to the mechanically induced stretch reflex. However, in this instance, the muscle spindles are bypassed by stimulating a mixed nerve directly. As a mixed nerve is stimulated, action potential volleys are sent in opposing directions along the afferent (spinal cord) and efferent (muscle) pathways. At lower stimulus intensities, the H-reflex can be mapped until reaching its peak amplitude as measured by surface EMG. As the stimulus intensity is increased, an opposing volley (antidromic) essentially masks the H-reflex, leaving behind the muscle response, or M-response. The H-reflex is interpreted within the context of sports medicine research as an estimate of alpha motoneuron pool excitability, or the proportion of alpha motoneurons available for use when normalized to the maximal M-response (H:M ratio). ${ }^{30}$ This measure has been extensively used to assess the motoneuron pool of the quadriceps ${ }^{85}$ and soleus ${ }^{21}$ musculature in healthy and injured cohorts, as well as the neural response following musculoskeletal injury. Although useful information can be gained from this measurement, it may be limited to monosynaptic synapses at the spinal level, and may miss a piece of the puzzle in regard to complete neurophysiologic assessment following joint injury. As this measurement is conventionally performed in a completely static state, due to inherent confounding during dynamic movement, it has the potential to bypass descending input from supraspinal centers. This provides a clearer interpretation of the measurement, but does not represent the complete neurophysiologic state of the individual.

\section{Corticospinal}

Although afferent signals project to the spinal cord directly, joint afferents are known to have extensive supraspinal projections to the cerebral cortex as well. ${ }^{86}$ Supraspinal influence on descending cortical output following injury is often neglected within the context of 
musculoskeletal research, specifically of the lower extremity, and has only begun to be better understood over the last decade. Cortical excitability has been researched in this regard following knee joint injuries. ${ }^{24,87}$ As descending pathways do have widespread projections within the spinal cord, there remains a great potential to influence AMI. Transcranial magnetic stimulation (TMS) provides a method of assessing excitability of the pre-motor area of the cerebral cortex. ${ }^{22} \mathrm{TMS}$ produces a small, but powerful field of magnetic energy that depolarizes neural tissue to initiate action potentials. $^{22}$ When a TMS device is placed over the scalp, superficial to the pre-motor area, action potentials are conveyed to the associated skeletal muscles resulting in a motor evoked potential (MEP). By stimulating the cortical neurons corresponding to quadriceps activity in the contralateral primary motor cortex, a motor evoked action potential can be detected via surface electromyography. When stimulated during minimal volitional activity of the involved musculature, this measurement is termed active motor threshold (AMT), and has been used as a primary indicator of corticospinal excitability in individuals following knee joint injury. ${ }^{88}$

TMS is a non-invasive tool used to measure neural conduction and processing time, activation thresholds, facilitation and inhibition in the primary motor cortex, and neural connections. ${ }^{22}$ Since its original description in 1985 , single pulse TMS has been widely used to study motor, visual, and somatosensory systems, as well as sensorimotor integration and cognition in patients with a variety of diagnosed disease processes. ${ }^{22}$ It has since emerged in sports medicine research as an intervention and assessment tool primarily in the upper extremity. ${ }^{89}$ Several authors have used TMS to measure cortical excitability in the lower extremities of varied cohorts. ${ }^{24,87,90,91}$ Our research laboratory has demonstrated the ability to successfully and reliably utilize this technique in the treatment ${ }^{90}$ and assessment ${ }^{91,92}$ of lower extremity muscular dysfunction. However, to date there is minimal evidence of the role of cortical excitability in neuromuscular recovery at various points in time following knee joint injury. 


\section{SENSORIMOTOR ADAPTATIONS TO JOINT INJURY}

Musculoskeletal injuries are common among athletic and recreationally active populations. Joint injuries specifically constitute a clinically important subgroup in these patient populations, presenting long-term consequences to joint health. Impairments in neuromuscular function and decreased self-reported quality of life have been reported in patients after ankle, knee, and hip joint injury. Strong evidence supports the link between lower extremity joint injury and early post-traumatic articular cartilage joint degeneration. ${ }^{9,12,13,93}$ Since articular cartilage degeneration is irreversible, the hallmark for prevention is early detection. Therefore, understanding the current knowledge base surrounding the neuromuscular adaptations that occur following joint injury is paramount.

Within the broad scope of the sensorimotor system, a term used to describe the sensory, motor, and central integration and processing components involved in maintaining joint homeostasis during bodily movements ${ }^{35}$, neuromuscular impairment has been purported to influence gross motor output, and should be of extreme interest to sports medicine clinicians. To effectively evaluate the neuromuscular function of an individual in this capacity, clinicians must have a comprehensive understanding of the neural adaptations that occur following injury. It is imperative that health care professionals continue to make efforts, from clinical practice to laboratory-based research, to identify objective evidence of persistent dysfunction following injury. This review will attempt to provide a link between clinicians and researchers from a measurement perspective in regard to neuromuscular changes following lower extremity joint injury.

\section{Measures of Neuromuscular and Sensorimotor Function}

The evolution of measurement techniques used to identify neuromuscular impairments following injury will be explored. Common clinical and laboratory-based measurement techniques used in current practice will be examined, with a specific focus on the application and interpretation of results. Within the literature, clear descriptions of measurement techniques and 
perspective on clinical interpretation related to lower extremity neuromuscular function are lacking. Therefore, the purpose of this review is to provide a detailed overview of the techniques used to evaluate neuromuscular function with specific regard to bridging the gap between common clinical and research specific measures of lower extremity function after joint injury.

\section{Use of Clinical and Laboratory Measures}

A variety of measurement techniques are commonly utilized in clinical practice to assess sensorimotor impairments following joint injury. Such measures provide valuable insight to gross functional impairments, which aid clinicians in assessing the progress of patients throughout the rehabilitation process. Many of the clinical assessment techniques utilized in practice have been validated using instrumented tools commonly used in the research setting. In addition, the ability of clinicians and researchers to reliably assess patients using these techniques and measurement tools has been established in most cases.

Laboratory based measurements are a vital component in sports medicine research, and beneficial supplement to clinical practice. Such tests are not meant to replace clinical measures, but to compliment them, in order to provide a comprehensive approach to clinical care.

Oftentimes, there may be a lack of interpretation in regard to the clinical relevance of laboratorybased measures, which can subsequently hinder the incorporation of such measures into clinical practice. The following section will attempt to provide an interpretation of commonly used clinically and laboratory measures in sports medicine research, while placing an emphasis on clinical relevance. Additionally, techniques and pitfalls associated with these measures will be highlighted.

\section{POSTURAL CONTROL}

Postural control is a multi-system process that relies on feedback from the somatosensory, visual, and vestibular input, and is a necessary component of daily function. ${ }^{94,95}$ Postural control, or balance, has been widely studied in musculoskeletal and cognitive injury research. These easy to use tests provide useful information to clinicians, and present good 
alternatives to more expensive laboratory based measures. Whereas static measures provide useful information in regard to postural control, they may not be challenging enough to detect impairments during physical activity ${ }^{96}$, and caution should be made when interpreting results. Numerous modifications have been described in the literature, making scoring and comparisons with other studies difficult. Results should be compared within individuals, ideally from preinjury data under the same testing conditions when possible.

\section{Clinical Measures}

\section{Balance Error Scoring System (BESS)}

The Balance Error Scoring System (BESS) is a clinically useful tool originally developed as a cost-effective objective assessment used in the evaluation of postural stability following concussion. ${ }^{95,97,98}$ As classically described, the BESS consists of 6 different conditions: doubleleg stance (hands on the hips with feet together), single-leg stance (standing on non-dominant leg with hands on hips), and a tandem stance (heel-to-toe with non-dominant foot behind) on a firm and foam surface, with the eyes closed and shoes removed..$^{95,99,100}$ This test is designed to detect gross balance deficits as noted by errors during each stance over a 20 second time epoch. Errors are defined as opening the eyes during stance, lifting the hands from hips, stepping, falling out of position, lifting the forefoot or heel, abducting the hip greater than 30 degrees or failing to return to the starting position in 5 seconds. ${ }^{99}$ Each error is given 1 point, with a maximum allowable number of 10 per stance. Once all 6 trials are completed, the total number of points per stance is summed, allowing for a maximum of 60 points. Point totals exceeding 10 in a given position, or 60 overall are considered a failed trial.

Deficits in postural stability are multi-factorial and common after joint injury. It is important not only to have an easy to use clinical tool, but a test that will be valid, reliable, and sensitive to change. The intra-rater ${ }^{97,101}$, inter-rater ${ }^{95,97,102}$, and test-retest ${ }^{103,104}$ reliability have been extensively examined. Although the BESS has been demonstrated to be sensitive enough to detect subtle postural changes, the reliability of this test is varied in the literature. ${ }^{99}$ Valovich McLeod et 
al. ${ }^{101}$ reported an intra-rater reliability ranging 0.87 to 0.98 ; however, a more recent review has reported 0.74 for total BESS scores.$^{97}$ Large ranges of inter-rater reliability from $0.57-0.96^{95,97,102}$ have been described for total BESS score, with test-retest reliability consistently lower between 0.64-0.67. ${ }^{103,104}$ Finnoff et al.$^{97}$ reported acceptable intra-rater reliability with firm single leg, firm tandem, and foam double leg, and inter-rater reliability for the firm single leg stance only. Additionally, a learned effect has been observed ${ }^{101}$, which has influenced how the test is administered. Broglio et al. ${ }^{103}$ demonstrated an increase in reliability when averaging three BESS trials within a given session, or twice if performed over multiple days. Furthermore, muscle fatigue has led to confounding results by increasing errors immediately after exercise. ${ }^{105}$ Therefore, it is recommended to wait a minimum of 20 minutes following exercise to administer the BESS. ${ }^{106}$ A modified version of the BESS was developed. Hunt et al.$^{107}$ described a modified version of the BESS using single-leg and tandem leg stance on firm and foam surfaces. Using this protocol, reliability was measured at 0.88 when averaging three trials. Due to the purported learning effect, the first trial was excluded from further analysis, reducing reliability slightly to 0.84. A subsequent analysis of one trial alone further reduced the reliability to 0.74 . An additional investigation of a modified protocol by Clark et al. ${ }^{108}$, using 6 conditions: single-leg dominant, single-leg non-dominant, and tandem on firm and foam surfaces, demonstrated fair test-retest and inter-rater reliability of 0.74 and 0.61 respectively. The results of these studies further support the recommendation for multiple trials when using either the original or modified BESS.

Beyond its use as a valid assessment tool for postural stability following sports related concussion, the BESS has been demonstrated to be a valid measure of assessing postural sway in individuals with functional ankle instability ${ }^{98}$, and sensitive to changes following fatigue ${ }^{105}$, neuromuscular training ${ }^{102}$, ankle bracing ${ }^{109,110}$, and varied training backgrounds. ${ }^{111}$ Additionally, subcategories of the BESS have been correlated with force plate measures, which are commonly used in the assessment of postural control following musculoskeletal injury. ${ }^{95}$ According to the presented literature, the BESS can be a valuable tool used in adjunct with other clinical and 
laboratory measurements in the assessment of sensorimotor impairment following musculoskeletal joint injury.

\section{Romberg Positional Stance}

Vestibular and somatosensory input are two of three peripheral modalities that influence static and dynamic postural control ${ }^{95}$, an aspect of sensorimotor control paramount in coordinated movement. Although vestibular dysfunction is not at the forefront of clinical sports medicine, a variety of clinical assessment techniques have been utilized to identify balance deficits in a variety of pathologic populations. Romberg's Test is a commonly used assessment tool described in the early $19^{\text {th }}$ century as a patient reported symptom to detect the loss of coordination due to a decrease in sensory input. ${ }^{112-114}$ This simple test may offer an important clue to the presence of vestibular dysfunction ${ }^{115}$, multiple sclerosis ${ }^{116}$, Parkinson's disease ${ }^{117}$, chronic low back ${ }^{118}$ and neck pain ${ }^{119}$, chronic ankle instability, and ACL injury. ${ }^{120}$ During the Romberg Test, the patient is asked to stand upright with feet close together, and arms at the side, in an anatomic position. ${ }^{113} \mathrm{~A}$ trial is initiated by having the individual stand still on a firm surface with the eyes open for a period of time, typically 10-30 seconds. During this time, the clinician will qualitatively note any deviations in movement. The individual's eyes are then closed, and the stance task is repeated, again noting any deviations in movement. The task can be altered by observing any postural abnormalities while having the individual look directly ahead and follow the clinician's finger as it moves rapidly from left to right or up and down. ${ }^{121}$ Variations have included a narrowed stance, tandem stance, balance on foam surfaces, use of footwear, alterations in hand placement, and external perturbations. ${ }^{113}$ Of recent, a modified Romberg Test of Standing Balance on Firm and Compliant Surfaces has been developed to identify isolated vestibular dysfunction. ${ }^{122}$ This modification includes 4 test conditions, adding double limb stance with the eyes open and closed

on a compliant surface to the originally described protocol. ${ }^{122,123}$ In addition to noting the degree of sway, the source (e.g. ankles, hips, entire body) should be recorded. To make appropriate observations, the clinician should stand directly in front of the individual being testing with arms 
extended to either side for support if necessary. A positive test, or Romberg's Positive, is determined if there is significant imbalance or worsening of balance with the eyes closed. ${ }^{121}$

Early descriptions of Romberg's Test have led to developments in modern posturography ${ }^{113}$, and variations of the test are currently utilized in a variety of medical disciplines. ${ }^{124,125}$ Within the realm of clinical sports medicine practice, studies assessing static postural control are most commonly reported with respect to ankle injuries. ${ }^{126}$ Although instrumented force plate measures have become the gold standard when assessing postural control, versions of Romberg's Test have been employed in clinical practice. In a 2008 systematic review, McKeon and Hertel reported that increased risk of sustaining an acute ankle sprain as noted by decreased postural control could be detected using a modified Romberg's test in conjunction with force plate measures. ${ }^{126}$ As decreased postural control has been demonstrated following ankle sprains, and linked with chronic ankle instability, the use of Romberg's Test during sensorimotor assessment following injury is warranted. Caution should be used when interpreting the results of this test, and ideally paired with baseline values; however, this may serve as a simple estimate of neuromuscular ability following lower extremity joint injury.

\section{Star Excursion Balance Test (SEBT)}

Dynamic balance is paramount in successfully completing athletic-related activities, and is frequently affected by musculoskeletal injury. Measures of dynamic postural control can be used to screen high-risk individuals or an assessment tool following injury as a means of identifying persisting dysfunction or return to play decisions. The Star Excursion Balance Test (SEBT) has been widely researched with regard to dynamic postural control. The SEBT was originally described as a rehabilitative tool ${ }^{127}$, and has multiple clinically relevant uses. The SEBT is a series of single-limb squats performed in eight directions on a flat surface using designated lines on the ground spread in $45^{\circ}$ angles. ${ }^{128}$ The non-stance limb is used to reach in each direction: anterior, anteromedial, anterolateral, medial, lateral, posterior, posteromedial, and posterolateral, which are named in orientation to the stance limb. The goal of the task is maintain 
a stable base of support with the stance limb with the foot flat of the floor surface, while reaching as far as possible with the non-stance limb. ${ }^{128}$ Athletic tape or a grid is commonly employed to measure reach distance; however, commercially available tools have been reported and may serve to improve the efficiency of the test. ${ }^{129}$ While reaching, the participant must touch down lightly with the distal most aspect of the reach limb lightly and return to the starting standing position. Should the reach limb rest of the floor surface, touch down hard, or lose balance, the trial is failed and should not be taken into consideration. ${ }^{128,130}$ The limb reach distance of 3 trials is typically averaged and normalized to limb length as measured from anterior superior iliac spine to medial malleolus, and expressed as a percentage of limb length. ${ }^{130}$ Normalization is most commonly performed using true limb length, and serves to standardize values for comparison between individuals.

Given that individuals unfamiliar with the task often perform poorly during initial trials, researchers have investigated the learning effect of the SEBT. An early investigation ${ }^{131}$ noted a plateau in reach distance after 7 trials, and therefore recommended participants perform 6 practice trials in each direction of the SEBT before recording. However, a more recent examination ${ }^{132}$ has recommended only 4 practice trials, which has been supported by follow up investigation. ${ }^{133}$ Although the accepted practice session has been reduced over time, the time necessary to complete testing in all 8 directions has led researchers to examine whether all directions serve a discrete purpose. In 2006, Hertel et al. ${ }^{134}$ demonstrated redundancy in participant performance in each of the 8 reach directions, which later led to the recommendation that only 3 reach directions: anterior, posteromedial, and posterolateral be performed. ${ }^{135}$ In a recent systematic review, Gribble et al. ${ }^{96}$ summarized all supported performance recommendations. Of note, clinicians are encouraged to conduct testing with the participant's shoes off, to use 4 practice trials prior to testing $^{132}$, to use video instruction when available, to control the order of testing, to standardize the position of the stance limb while minimizing movement of the foot and trunk during reach ${ }^{136}$, 
maintain the participant's hands on hips ${ }^{136}$, and to normalize reach distance to limb length of the stance leg. ${ }^{130}$

To further support its clinical use, strong intra-rater reliability using the SEBT has been reported. Kinzey and Armstong ${ }^{109}$ first reported intra-rater reliability of the SEBT, at a range of 0.67 to 0.87 , whereas Hertel et al. ${ }^{131}$ later noted an increase, from 0.81 to $0.96\left(\mathrm{ICC}_{2, \mathrm{k}}\right)$. The SEBT has also been demonstrated as a reliable measure between clinicians, with inter-rater reliability ranging 0.35 to 0.84 and 0.81 to $0.93 .{ }^{127}$ Although 0.35 is not an acceptable level of reliability, this value was determined on the first day of a two-day testing session, and has been attributed to a learning effect. ${ }^{127}$ Beyond the established reliability of this tool, it has demonstrated validity as a dynamic test to predict risk of lower extremity injury ${ }^{129,137}$, to identify dynamic balance deficits following a variety of lower extremity injuries ${ }^{134}$, and to detect postural responses to neuromuscular training programs in healthy and injured individuals. ${ }^{96,102,138}$

In light of the available evidence, the SEBT can be considered a highly representative non-instrumented dynamic balance test for physically active individuals. ${ }^{96}$ The SEBT combines sagittal, frontal, and transverse moments, while allowing for an easy to use, and cost-effect approach to the neuromuscular assessment. Although this is a widely utilized assessment tool, caution should be made when interpreting results. With conflicting evidence of varied effects based on sport, sex, age, foot type, time of day, and body height, efforts should be made to standardize between session trials. Furthermore, the SEBT should be used in conjunction with other clinical assessment tools, and not solely relied upon when assessing sensorimotor function.

\section{Laboratory Measures}

\section{Forceplate}

Postural control deficits have been widely observed following lower extremity injury. ${ }^{139-}$

${ }^{146}$ Such impairments are commonly assessed in research settings via changes in balance and distribution of pressure about the foot. Instrumented measures using force plate and pressure mat calculations are useful in describing these impairments with high precision. Although these 
measures have been largely studied among individuals with chronic ankle impairments, such data are not limited to those suffering from distal joint injuries.

Traditional force plate measures including average center of pressure (COP) excursion velocity and area have been used to describe changes in postural control following injury ${ }^{126}$ and rehabilitative programs. ${ }^{147}$ These measurements are calculated during a single limb balance task, and provide information in regard to how quickly the foot is moving, and the size of area covered during movement, with higher values of each indicating poorer performance. A more recent approach to characterizing postural control is a time-to-boundary (TTB) analysis. ${ }^{148-150}$ TTB is a spatiotemporal analysis of COP data points that quantifies the theoretical amount of time an individual has to make a postural correction in order to maintain postural stability. ${ }^{148-150}$ In this instance, lower values are considered pooper outcomes, indicating less time to make postural corrections. Wide, albeit acceptable levels of reliability, have been reported using traditional $\left(\mathrm{ICC}_{2,1}=0.35-0.80\right)$, and TTB $\left(\mathrm{ICC}_{2,1}=0.34-0.87\right)$ measures of postural stability between sessions. ${ }^{150}$ Understanding how well an individual can respond to changes in postural demands is a primary point of concern for clinicians when determining readiness for activity progression following injury.

Alterations in the distribution of plantar pressures have been observed following acute and chronic ${ }^{151-154}$ lower extremity joint injury. Most notably, the shift towards a more laterally based COP has been observed during gait pattern among those with a history of chronic ankle instability. Researchers have used pressure mats and instrumented insoles to identify shifts in these pressures following injury. Utilizing, or understanding, the interpretation of such instrumented measures may aid clinicians in identifying sensitive changes during this process. 


\section{FUNCTIONAL TASKS}

\section{Clinical Measures}

\section{Landing Error Scoring System (LESS)}

Non-contact ACL injuries remain a major concern in sport and carry with them long-term sequelae. ${ }^{155,156}$ The high prevalence of ACL injuries have led to a vast amount of research related to faulty movement patterns, ACL risk and prevention, and ACL loading characteristics. ${ }^{51,157-161}$ The available evidence has demonstrated an inter-relationship between these factors, specifically between lower extremity movement patterns and ACL loading. ${ }^{162}$ Such data has led to a relatively consistent list of ACL risk factors and the development of subsequent prevention programs. Isolated and combined patterns of knee valgus, internal rotation, and decreased flexion have been traditionally labeled as high-risk movement patterns for non-contact ACL injury. ${ }^{162,163}$ Additionally, faulty movements distal and proximal to knee joint have been related to lower extremity injury risk. ${ }^{160}$ In light of the importance placed on optimal movement patterns during sport, and the ability to correct faulty movement patterns efforts have been made to establish clinically relevant tools to identify high-risk individuals.

The Landing Error Scoring System (LESS) is a qualitative assessment tool used to identify gross movement pattern dysfunction. Padua et al. ${ }^{160}$ developed this tool as an inexpensive method of providing a standardized functional movement assessment. The LESS uses two offthe-shelf video cameras to record motion in the sagittal and frontal planes during a jump landing maneuver. Each camera is set 136 inches from the landing area facing each plane, and 48 inches from the floor. To perform this task, the individual must jump with both feet from a $30 \mathrm{~cm}$ high box to a distance of $50 \%$ of their height away from the box. The individual is instructed to jump from a neutral position with feet shoulder width apart and toes pointing forward, and perform a maximal vertical jump immediately upon landing. Evaluator instruction during testing is minimal, with feedback provided only to insure the task is performed correctly. Several practice trials are commonly allowed, with the average of three trials taken. ${ }^{160,164,165}$ 
LESS scores are simply reflective of gross observable movement errors during a jumplanding task, with higher scores indicative of high-risk biomechanics. ${ }^{160}$ Trials are scored in the sagittal and frontal planes, on a 17-item scale. Scoring is broken down into several sets of items: (1) lower extremity and trunk position at time of initial ground contact, (2) positioning of the feet at initial ground contact, (3) lower extremity and trunk motion between initial ground contact and maximum knee flexion, and (4) overall sagittal plane motion and general perception of landing quality. Total scores can be dichotomized into excellent $(\leq 4)$, good $(>4$ to $\leq 5)$, moderate $(>5$ to $\leq 6)$ and poor $(>6)$ landing techniques. ${ }^{160}$ In the absence of an injured extremity, the dominant limb is focused on for scoring purposes. Padua et al. has reported good inter-rater and excellent intra-rater reliability of 0.84 and 0.91 respectively. ${ }^{160}$ Authors employing large-body screening interventions have demonstrated excellent inter-rater reliability $\left(\mathrm{ICC}_{2,1}=0.835\right)$ between expert and novice raters in scoring the LESS, which further supports its use in a variety of clinical settings. ${ }^{166}$

In addition to the LESS, researchers have used 2D video analysis to measure frontal plane motion during other common athletic tasks. The use of 2D video analysis has been validated $^{167}$ and demonstrated to be a reliable measure of lower extremity dynamic knee valgus. ${ }^{168}$ Although simple video analysis is inherently useful beyond the scope of the described LESS criteria, the LESS is recognized as the most commonly used valid assessment tool to identify dynamic movement error. In 2009, Padua et al. ${ }^{160}$ validated the LESS against the gold standard of 3D motion capture analysis. However, in cases in which 2D video cameras are unavailable and/or immediate feedback is warranted, a real-time version of the LESS (LESS-RT) has been explored. In 2011, Padua et al. ${ }^{165}$ reported on the inter-rater reliability of the LESS-RT, ranging $0.72-0.81$. In this modified version, individuals complete 4 trials of the previously reported jump-landing maneuver, and are evaluated on 10 jump-landing characteristics by two raters in the frontal and sagittal planes. Despite showing promising evidence of clinical implementation, the LESS-RT has yet to be validated to the same extent as the original LESS. 
As the development of clinical tools to predict injury, or risk of injury, attempts to become more sophisticated, a variety of field-based algorithms have recently been described. With the demonstrated link between increased knee abduction moments (KAM) during landing, specifically in females ${ }^{169}$, efforts have focused on prevention programs to reduce knee valgus. In 2012, Myer et al. ${ }^{170}$ described a field-based measurement used to predict high knee abduction moments in female athletes who may be at greater risk of injury. This algorithm utilized a jumplanding task similar to the LESS, taking into account measures of body mass, tibia length, quadriceps to hamstrings ratio, knee valgus motion, and knee flexion ROM. The described algorithm accounted for $78 \%$ of the variance in KAM during landing, and was able to successfully predict high KAM with $85 \%$ sensitivity and $93 \%$ specificity. Although algorithms may aid clinicians in the absence of more sophisticated measurement instruments, caution should be taken when applying them to larger populations. Authors have questioned the utility of such measures, conducting a large, prospective study that found the probability of high knee abduction moments was not associated with noncontact ACL injury in at risk female high school and collegiate athletes, or matched healthy individuals. ${ }^{171}$ Such field-based assessments can be easily implemented within clinical practice; however, extrapolation of predicted kinetics should be interpreted with caution.

Although follow up examination of the LESS has demonstrated an inability of the test to predict ACL injury ${ }^{172}$, it has been shown to detect kinematic changes linked with ACL injury risk factors. ${ }^{159}$ The primary aim of the LESS is to identify faulty movement patterns during the early landing phase, in which individuals are at higher risk for injury. The LESS specifically identifies high-risk patterns previously linked with ACL injury from a gross perspective. Traditionally, scores have been used as a screening assessment to implement prevention programs for large athletic cohorts. ${ }^{157,159,166}$ However, given the nature of its intent, the LESS may be a beneficial assessment tool along the spectrum of rehabilitation following a lower extremity joint injury. Furthermore, implementing the LESS into return to play criteria would inherently serve clinicians 
well, providing them an additional criterion for progression. With specific recommendations including quantification of muscle strength, stability, neuromuscular control, and function following ACL reconstruction ${ }^{156}$, the LESS may be a valuable tool used throughout the rehabilitative process.

\section{Step-Down Tests}

Step-down tasks are commonly used in clinical practice to assess lower extremity movement patterns and general quality of movement. ${ }^{173}$ The lateral and frontal step-down are useful tasks that pose a challenge to the strength and neuromuscular control of the lower extremity, which can be useful as an assessment tool before and after injury occurs. ${ }^{174}$ With the demonstrated link between poor dynamic stability, specifically in the frontal and transverse planes, and increased risk of lower extremity injury ${ }^{51,169}$, it remains highly important for clinicians to employ easy to use tests to identify neuromuscular dysfunction.

Step-down tasks may be used to identify poor dynamic alignment in the frontal and transverse planes, including excessive pelvic drop, hip adduction and internal rotation, knee valgus, and foot pronation. ${ }^{175,176}$ These tasks essentially utilize a controlled, eccentric single limb squat with the addition of a contralateral heel touch. Individuals being tested are asked to stand naturally in single limb support with hands on the waist on a raised box in the range of $20-31 \mathrm{~cm}$ in height. ${ }^{177,178}$ Some authors have recommended using a height that allows for participants to achieve 60 degrees of knee flexion on the stance limb. ${ }^{179}$ Participants are then asked to slowly lower the opposite foot at a self-selected pace to lightly touch the floor with the heel, and return to the previously standing position. ${ }^{173}$ Practice trials have been reported used to familiarize participants with the task. Multiple trials (i.e. 5-6) are performed when assessing the quality of movement. Trials are scored based on 5 criteria: 1) Arm strategy, 2) Trunk movement, 3) Pelvis plane, 4) Knee position, and 5) Steady stance. ${ }^{177,179}$ For a more detailed description of grading criteria, refer to Piva et al. ${ }^{177}$ Possible scores may range $0-7$, with higher scores indicating poorer quality of movement. Some authors ${ }^{177}$ have dichotomized scores into ranges, indicating good (0- 
1), medium (2-3), and poor (4+) quality; however, the risk of lower extremity injury per category remains unknown.

Given the prevalence of step-down tasks in clinical practice, authors have attempted to assess the reliability in healthy cohorts. Acceptable intertester reliability of 0.67 has been demonstrated with the lateral step-down task, using the aforementioned scoring criteria. ${ }^{177}$ However, when using a global qualitative approach to observing quality of movement, authors ${ }^{178,180}$ have reported lower intertester and intratester reliability. To improve the accuracy of qualitative observations during these tasks, simply strategies, such as marking the tibial tuberosity to facilitate visualization can be employed. ${ }^{179}$ Although less clinically applicable, 3D motion capture has been used as a supplement to quantify movement patterns during step-down tasks.

When deciding whether to incorporate step-down tests from a screening (pre or post injury) or rehabilitative perspective, clinicians should consider the joint forces created, and the specific biomechanical components to be assessed. Greater patellofemoral joint reaction forces have been described in both frontal and lateral step-down tasks as compared to those stepping up. ${ }^{181}$ Understanding the kinematic influence on joint reaction forces will help guide clinicians when implementing this these tasks. Additionally, the lateral step-down task has been reported to be a more useful tool when assessing neuromuscular control of the hip, whereas a drop-vertical

jump may detect greater frontal plane changes at knee. ${ }^{173}$ Although step-down tasks may be used to assess gross lower extremity quality of movement, appropriate caution should be taken when making clinical decisions.

\section{Single Limb Hop Tests}

Criterion-based measures are important pieces of the rehabilitation process. ${ }^{156,182}$ From a functional perspective, this becomes extremely relevant when making return to play decisions. The clinician is often faced with the task of matching an appropriate task, based on difficulty and functionality, to an individual following an injury. Some dynamic tests may not challenge the participant enough to adequately identify deficiencies. In late stages of rehabilitation, dynamic 
tasks should be difficult enough to identify neuromuscular adaptations that an individual will likely experience during sport. Additionally, using such tasks during early phases of rehabilitation may be useful in predicting functional outcomes after non-operative injuries. Single-leg hop tests have been commonly used to evaluate functional performance specifically after ACL injury or reconstruction. ${ }^{183}$

Single-leg hop tests are commonly used in a rehabilitative setting as measures of function and impairment ${ }^{183}$; however, these tests encompass a variety of tasks, making comparisons between individuals or groups difficult at times. Several more commonly used hop tests have been described by Noyes et al. ${ }^{182}$, and include the single hop for distance, triple hop for distance, crossover hop for difference, and 6-meter timed hop. ${ }^{184}$ Albeit others have been described, such as the hop and stop ${ }^{185}$, stair hop ${ }^{186}$, and vertical jump tests ${ }^{187}$, the focus of this review will remain on those previously mentioned given their demonstrated reliability and prevalence in functional testing. ${ }^{184,188,189}$

The goal of the single, triple and crossover hop tests is to achieve maximum hop distance moving forward while maintaining a controlled landing strategy on the ipsilateral limb. ${ }^{184}$ During the timed hop test, the goal is to hop as quickly as possible over a 6-meter distance. ${ }^{184}$ In each test, participants should not be instructed to restrict arm movement, and should maintain the final landing for a minimum of 2 seconds to verify jump distance. A loss of balance, additional hop, or touching down of the contralateral limb is considered a failed trial. Measurements are be made from the great toe to the rear of the foot upon landing using a tape measure. Raw scores are recorded in centimeters based on the maximum hop distance achieved, and normalized to limb length when making comparisons between individuals. ${ }^{190}$ Scores are most commonly reported in context of limb symmetry as a percentage. ${ }^{156,182,183}$ Limb symmetry has been described as an important prognostic factor after injury, with higher hop symmetry indices being predictive of knee function following ACL reconstruction. ${ }^{191}$ The limb symmetry index (LSI) is commonly used in functional assessments following injury, and can be described as: [(involved 
score/uninvolved score) $x 100 \%$ ]. A LSI of $\geq 85$ has been described as 'normal' limb

symmetry ${ }^{182}$; however, recent evidence suggests normal values are greater than 90 (cite).

To enhance its use in clinical practice, authors have extensively studied the reliability of hop tests, reporting intraclass coefficients from 0.66 to $0.99 .{ }^{190,192,193}$ Good to excellent test-retest reliability has been demonstrated in the single-leg, triple-leg, and cross-over hop test. ${ }^{190}$ Early investigations of reliability may be underestimated due to a learning effect that has been described in more recent investigations. Previous authors ${ }^{192}$ have recommended three practice trials be used for the four mentioned hop tests, which has been corroborated by follow up study. ${ }^{190}$ However, due to its complexity, a fourth practice trial has been recommended when performing the crossover hop test. ${ }^{190}$ Gender may also be considered when determining how much practice to include prior to testing, as limited evidence has demonstrated that males require less practice than their female counterparts when performing the single-leg timed hop test. ${ }^{190}$ Despite these claims, familiarity and complexity of the task should be used to determine the quantity of practice trials, with a minimum of three practice trials included.

Hop tests have are used ubiquitously in clinical practice as a measure of functional ability following injury. Function is an important outcome to patient satisfaction after injury, and has specifically been reported on following ACL reconstruction. In an attempt to move towards evidence-based practice, it is important that patient oriented outcomes, such as self-reported function, are included in clinical care. Hop tests have been used in such a manner, and continue to help clinicians understand functional capabilities. They have been reported as sensitive tests, able to detect difference between limbs after lower extremity injury, helping to identify limb asymmetries. Additionally, hop tests have been able to predict strength and power in healthy individuals. Furthermore, these tests have been researched in a variety of populations, owing to the generalizability of results within athletic cohorts. Although hop tests may partially fill a gap between mid-stage rehabilitation and return to sport, they should be used in conjunction with 
other clinical and laboratory measures to capture neuromuscular performance in a comprehensive manner.

\section{Laboratory Measures}

\section{Kinematic/ Kinetic Measures}

Gait adaptations have been observed in individuals following an induced knee joint effusion $^{20}$, ACL tear ${ }^{194,195}$, and ACL reconstruction. ${ }^{196}$ Compensatory movement strategies in the sagittal plane, most notably reduced knee flexion angles evident by a decrease in external knee extension moment, has been observed following ACL injury. ${ }^{194,197}$ These adaptations have been theorized to be the result of decreased muscle strength ${ }^{198}$ distal and proximal to the injured joint. Additionally, muscular imbalances have been reported as a possible contributor to a quadriceps avoidance pattern. ${ }^{199}$ The combination of decrease muscular strength and reduced joint angles results in a less effective mechanism of load absorption during activity while preserving functional mobility. The reduced ability to dissipate loads, results in greater forces directed through the joints of the lower extremity. Researchers have employed a variety of motion analysis techniques in order to capture kinematic and kinetic adaptations before and after joint injury.

Three-dimensional motion analysis has been largely utilized in sports medicine research to measure gait related kinematics. In doing so, camera-based systems such as Vicon, are routinely used. Camera-based systems require the use of numerous retro reflective markers placed along body segments of interest, which can be detected during motion. This form of motion capture allows researchers and clinicians to utilize a large space, able to accommodate a variety of physical tasks. However, this system relies on maintaining visualization of placed markers. Therefore, marker security, participant clothing and body composition become important considerations during the set up process.

In contrast to conventional camera-based systems, electromagnetic motion capture allows for an alternative method of measuring gait related kinematics. These systems operate by projecting a spherical electromagnetic field, which detect sensors placed on body segments. In 
contrast to the previously mentioned system, sensors placed on the body are not free, but connected to a central unit. Additionally, any functional tasks completed are limited to the projected field, which is typically in the range of 10 meters. Although this system may utilize a smaller area in which to complete a given task, electromagnetic systems are portable, and can be used to measure kinematics during filed based activities.

Although a variety of methodological considerations exist when performing 3D motion capture analysis, many of the common kinematic measures obtained have been reported to be reliable. In a 2009 systematic review of studies examining the inter-session and inter-assessor reliability of three-dimensional kinematic gait analysis, McGinley et al. ${ }^{200}$ reports high reliability indices $(\mathrm{CMC}=0.83$ to 0.99$)$ at the hip, knee, and ankle in the sagittal plane. Additionally, the least amount of errors were found in pelvic rotation, pelvic obliquity, and hip abduction. However, low reliability and increased errors were reported to occur during hip and knee transverse plane motion. Despite the observed decrease in reliability in the transverse plane, this review ultimately concluded that clinically acceptable errors are possible in gait analysis.

\section{MUSCLE STRENGTH AND ACTIVATION}

\section{Clinical Measures}

\section{Hand-Held Dynamometry}

Force based measures are commonly used in clinical practice during the evaluation and rehabilitation process. In the clinical setting, force based measures are almost exclusively limited to manual resistance and hand held dynamometry testing. Although, clinics may have isokinetic testing available, this will be discussed in later sections in more detail.

Hand-held dynamometry (HHD) is a commonly used clinical measure to quantify isometric strength. In addition to its use clinically in sports medicine practice, HHD has been used to examine muscle strength in a variety of pathologic cohorts, including cerebral palsy ${ }^{201}$, spinal cord injury ${ }^{202}$, and traumatic brain injury. ${ }^{203}$ With questionable reliability of such measures reported in these cohorts, recommendations have been developed to improve the validity and 
reliability of HHD measurements within healthy and athletic populations. ${ }^{204,205}$ Although handheld dynamometry has demonstrated good intra-rater reliability, its use has been limited in its inability to produce reliable data between testers $(\mathrm{ICC}=0.11-0.28)$, due to varying counter pressure applied. ${ }^{206}$ Additionally, the subject strength or mechanical advantage over the tester may confound results. In response to poorly observed reliability between examiners, authors ${ }^{206}$ have recommended the use of a novel, resistance-enhanced dynamometer to standardize force production. These authors have reported strong intra-examiner $(0.91-0.94)$, inter-examiner $(0.98)$, inter-session $(0.91-0.92)$, and intra-session $(0.93-0.99)$ reliability using this approach. Although such recommendations may enhance the reliability of hand held measures, they may not always be practical.

Despite previous reports questioning the reliability of standard hand-held dynamometry, more recent authors ${ }^{204}$ have cited acceptable reliability between sessions $(\mathrm{ICC}=0.62-0.92)$, between testers $(\mathrm{ICC}=0.65-0.87)$, and within tester $(\mathrm{ICC}=0.77-0.97)$ when using a systematic approach to measure lower extremity strength in young, healthy individuals. Furthermore, this study provided evidence that the level of tester experience had little to no bearing on the intratester reliability.

While acceptable values have been reported, the use of HHD is indicated in early stages of recovery following injury, and is not recommended as a primary strength measurement in healthy, strong individuals. ${ }^{204,203}$ Despite its inherent limitations, HHD presents a viable option in quantitative strength assessment following injury. Its portability, generalizability between clinicians and easy of use make this an attractive tool in sports medicine practice. ${ }^{204,207}$

\section{Laboratory Measures}

\section{Maximal Voluntary Isometric Contraction (MVIC)}

Instrumented force based measures are commonly utilized for clinical and research purposes in the evaluation of strength following joint injuries. These data are conventionally used as criteria for return to play decision-making, and are often heavily relied upon as objective 
measures. ${ }^{156}$ With the established link between decreased muscle strength following joint injury and poor outcomes ${ }^{67,208}$, multimode dynamometry is widely used to assess persistent weakness. Strength assessments are conventionally performed bilaterally, with the uninjured limb serving as a reference standard. In certain cases, this method may serve as an adequate comparison; however, the presence or past history of injury to the contralateral limb may hinder its use in this capacity. Furthermore, authors have demonstrated evidence of bilateral neuromuscular deficits following acute unilateral joint injury. ${ }^{24,209}$ To contribute to this notion, these deficits have been cited to persist into chronic phases of healing, becoming a plastic central nervous system adaption is left untreated. ${ }^{1}$ Therefore, in addition to using force-based measures in isolation, researchers have begun to explore more advance techniques of neuromuscular assessment following joint injury. $^{87}$

Instrumented force based estimates of torque have demonstrated strong reliability when repeated within and between sessions in the lower extremity. Authors have reported intraclass correlation coefficients of ranging $0.93-0.96$. Although isokinetic dynamometry may not provide all information necessary to the clinician's decision-making process following injury, it will provide a substantial estimate of persistent asymmetries between limbs.

\section{Estimates of Volitional Muscle Activation}

Voluntary activation failure is a common occurrence following lower extremity joint injuries, resulting from the inability of the central nervous system to provide maximal descending input to a muscle during volitional contraction..$^{33,89,210}$ Quadriceps central activation failure is common following knee joint injury ${ }^{1,26,67,211,212}$ and has been described as a significant predictor of post-traumatic osteoarthritis if left untreated..$^{1,26,93,213}$ Furthermore, neural changes in the musculature of the lower leg have been observed in the presence of chronic ankle joint injury. Given the nature of these consequences to manifest as irreversible degenerative changes with long-term joint health implications, it is important to have valid estimates of central activation in this context. 


\section{Interpolated Twitch Technique (ITT)}

The interpolated twitch is a technique commonly employed to estimate skeletal muscle activation during voluntary effort. ${ }^{214}$ Originally described by Merton ${ }^{215}$, the ITT came to light under the hypothesis that the force produced by voluntary effort is limited by the capacity of the central nervous system. It was this thought that led to the notion of using a supramaximal exogenous agent, electrical stimulation, to recruit inhibited motor units in an attempt to estimate true muscle activation. The ITT was the first technique popularized in this context.

Developments in this technique have demonstrated an inability to fully activate all muscle fibers in healthy individuals, which have propagated its investigation in musculoskeletal research since.

The ITT requires the delivery of a supramaximal exogenous agent with the ability to excite inhibited musculature that cannot be volitionally activated. ${ }^{216,217}$ This technique involves direct stimulation of the nerve trunk innervating the muscle being studied (e.g. femoral nerve; quadriceps) via focal stimulating electrode, or intramuscular nerve branches of an active muscle during voluntary contraction (e.g. quadriceps muscle belly). ${ }^{214}$ Delivery of a $200 \mathrm{~V}$ single or doublet electrical pulse generates a transient twitch response, which provides an estimate of muscle activation, or percent inhibition. ${ }^{217}$ In this instance, the size of the resultant twitch is indicative of the degree of inhibition present, with greater twitches indicating more inhibition. Stimuli are delivered with the muscle in an active and relaxed state. The resultant twitch generated during each state can be used to estimate voluntary activation as a percentage using the linear equation: [1-(superimposed twitch/control twitch)] x 100. In this instance the superimposed twitch refers to the stimulus being delivered in concert with a voluntary muscular contraction, and the control twitch being that obtained in a resting state.

Although equations such as these may provide useful insight to neuromuscular capacity,

evidence has demonstrated a non-linear relationship between evoked and voluntary force. ${ }^{216,218,219}$ This finding has led researchers to question the validity of using such ratios and to rather extrapolate the evoked-voluntary force relationship, and estimate 'true activation.' For more 
information on this technique, refer to Shield and Zhou. ${ }^{214}$ Reliability of the ITT has improved with methodological advances. Authors have reported acceptable intraclass correlation coefficients in the biceps brachii $(0.858$ [95\% CI $0.61-0.98])$ using a single electrical pulse ${ }^{220}$, and soleus musculature ( 0.52 to 0.84$)$ using a doublet stimulus. ${ }^{221}$ However, improvements in reliability (ICC $\geq 0.74$ ) have consistently been noted when paired with a voluntary contraction greater than $40 \%$ of MVIC..$^{218,221,222}$

Technological advances have improved the sensitivity of the ITT over time. Despite these advances, a number of limitations of this measure persist. The greatest limitation is the assumption that a percutaneously delivered supramaximal stimulus will recruit all motor units (MU) unable to be volitionally recruited, and likewise that the control twitch measured in a resting state provides an estimate of the complete motor neuron pool. ${ }^{214,217,218,223-225}$ Although this technique has demonstrated the ability to recruit MUs beyond those which can be recruited volitionally, we cannot distinctly state that complete activation occurs. Authors have attributed the inability to maximally recruit MUs to the antidromic volleys that occur when stimulating a mixed motor nerve, and have postulated that these factors result in a net overestimation of muscle activation. ${ }^{226}$ Additional limitations including participant discomfort, impedance from subcutaneous tissue, and the observed non-linear relationship between evoked and voluntary forces force researchers and clinicians to interpret results with caution. ${ }^{214,218}$

Although limitations to this measure exist, it has been utilized in a variety of sports medicine research to estimate muscle activation during and after bouts of fatigue, maximum force production capability, to evaluate the influence of resistance training. Additionally, the ITT and similar measures have been employed to evaluate patients following joint injury and subsequent disuse. ${ }^{214}$ Despite the inherent limitations of this technique, valuable information in regard to gross muscle activation relative to one's maximal potential may still be gained. Measurements should be interpreted with caution, but not discounted. 


\section{Superimposed Burst Technique (SIB)}

The superimposed burst technique (SIB), likewise to the ITT, requires a percutaneously delivered supramaximal agent to provide an estimate of volitional muscle activation. Since it's early descriptions, the SIB technique has become increasingly popular in musculoskeletal research. ${ }^{33}$

In contrast to the ITT, the SIB technique however provides a train, or burst, of electrical stimuli via two stimulating electrodes directly over the quadriceps musculature at the proximal lateral and distal medial during a maximal voluntary knee extension task. By superimposing a supramaximal stimulus in this fashion, a transient twitch in force production is generated, indicating the maximal capacity of the motoneuron pool. The percent of volitional muscle activation can then be estimated and expressed as a ratio. The central activation ratio (CAR) is an equation commonly used to estimate maximal volitional activation of the quadriceps musculature. ${ }^{33,38}$ Conventionally, the SIB technique is used in this manner to deliver a supramaximal percutaneous electrical stimulus to the quadriceps musculature during a maximal voluntary isometric knee extension task $\left(\mathrm{T}_{\mathrm{MVIC}}\right) \cdot{ }^{33,227}$ The resultant torque $\left(\mathrm{T}_{\mathrm{SIB}}\right)$ from this technique can be interpreted as a theoretical representation of the complete motoneuron pool. ${ }^{33}$ Therefore, CAR measurements of 1.0 , or $100 \%$ if expressed as a percentage, are indicative of complete volitional activation $\left(\mathrm{CAR}=\mathrm{T}_{\mathrm{MVIC}}\left[\mathrm{T}_{\mathrm{MVIC}}+\mathrm{T}_{\mathrm{SIB}}\right] \times 100\right) .{ }^{33,38}$

Despite early beliefs that maximal recruitment of all motoneurons was possible, researchers have since demonstrated an inability to maximally activate all muscle fibers during voluntary effort. Through these observations and CAR studies, 0.95 , or $95 \%$, activation has been deemed fully activated. ${ }^{228}$ This value is somewhat arbitrary however, as other authors have reported values between 0.84 and 0.99 . Despite these data, debate in regard to normative CAR values in healthy individuals has remained prevalent, with methodological variations the likely culprit for these differences. 
Although methodological variations persist within this measurement, acceptable levels of reliability have been established. The superimposed burst technique has been recognized as a reliable measure of quadriceps activation in the open chain position, with inter-session intraclass

correlation coefficients $\left(\mathrm{ICC}_{2, \mathrm{k}}\right)$ ranging 0.80 to $0.86 .{ }^{229,230}$ Even greater within session reliability has been demonstrated in young healthy individuals $\left(\mathrm{ICC}_{2,1}=0.94\right)$. It is likely that a variety of factors, including verbal encouragement, task instruction, and joint angle during testing play a significant role in CAR values. Additionally, the SIB technique has been reported to overestimate muscle activation when compared to the gold standard of MRI based studies. Despite the various considerations clinicians and researchers must take into account, the SIB serves as a viable option to estimate volitional muscle activation before and after joint injury.

\section{CENTRAL NERVOUS SYSTEM EXCITABILITY}

\section{Estimates of Spinal Reflex Excitability}

\section{Hoffmann Reflex (H-Reflex)}

Research to date has largely focused on spinal mechanisms of muscle inhibition following joint injury. ${ }^{231}$ The Hoffmann reflex (H-reflex) is a common neurophysiologic test used in sports medicine research to assess modulation of monosynaptic reflex activity in the spinal cord. ${ }^{30}$ The H-reflex can be viewed as analogous to the mechanically induced stretch reflex. However, in this instance, the muscle spindles normally activated are bypassed by stimulating a mixed nerve directly. As a mixed nerve is stimulated, action potential volleys are sent in opposing directions along the afferent (spinal cord) and efferent (muscle) pathways. At lower stimulus intensities, the H-reflex can be mapped until reaching its peak amplitude as measured by surface EMG. As the stimulus intensity is increased, an opposing volley (antidromic) essentially masks the H-reflex, leaving behind the muscle response, or M-response. The H-reflex is interpreted within the context of sports medicine research as an estimate of alpha motoneuron pool excitability, or the proportion of alpha motoneurons available for use when normalized to the maximal M-response $\left(\mathrm{H}_{\max }: \mathrm{M}_{\max }\right)$, which represent the total motoneuron pool available. ${ }^{30}$ 
This measure has been extensively used to assess the motoneuron pool of the quadriceps $^{85,232,233}$ and soleus ${ }^{21,234,235}$ musculature in healthy and injured cohorts, as well as the neural response following musculoskeletal injury. Although useful information can be gained from this measurement, it may be limited to monosynaptic synapses at the spinal level, and may miss a piece of the puzzle in regard to complete neurophysiological assessment following joint injury. As this measurement is conventionally performed in a completely static state, due to inherent confounding during dynamic movement, it has the potential to bypass descending input from supraspinal centers. This provides a clearer interpretation of the measurement by limiting pre-synaptic inhibition, but does not likely represent the complete neurophysiologic state of the individual.

The H-reflex has been widely used in sports medicine research to identify the state of excitability along the spinal tract following musculoskeletal injuries ${ }^{21}$, effects of therapeutic modalities ${ }^{236}$, and pain. ${ }^{237}$ Additionally, the H-reflex has been used to examine the effects of exercise training ${ }^{173}$ and performance of motor tasks ${ }^{238}$ in healthy and injured individuals. Albeit useful information may be gained from this neurophysiologic technique, it carries with it many inherent methodological considerations. This measure is extremely sensitive to the influence of pre-synaptic inhibition from supraspinal centers of the body. ${ }^{30,239}$ Therefore, subject positioning, lighting, time of day, and stress may each affect H-reflex values. Additionally, considerations in regard to the specific instrumentations such as stimulation setup, duration, and intensity make this technique difficult to reproduce between studies. ${ }^{30}$ However, strong intersession $\left(\mathrm{ICC}_{3,1}=0.97\right)$ and intrasession $\left(\mathrm{ICC}_{2,1}=0.97\right)$ reliability has been reported in the quadriceps ${ }^{240}$, where this measure is typically taken. Additionally, strong and acceptable levels of reliability have been demonstrated for the $\mathrm{H}: \mathrm{M}$ ratio between sessions in the soleus $\left(\mathrm{ICC}_{2,1}=0.97\right)$, peroneals $\left(\mathrm{ICC}_{2,1}=\right.$ $0.97)$ and tibialis anterior $\left(\mathrm{ICC}_{2,1}=0.78\right) .{ }^{241}$ In addition to these factors, the greatest limitation of the H-reflex measure is that is an electrically induced reflex, and not one that occurs naturally in the human body. 
Despite this limitation, it can provide insight to the neural pathways from peripheral areas of the body to the spinal cord following injury. However, caution should be taken when interpreting H-reflex measures. If care is taken to obtain valid measurements, the H-reflex can provide information about the state of neuromuscular function following injury.

\section{Volitional Wave (V Wave)}

The volitional wave (V-wave) is an electrophysiological variant of the H-reflex, ${ }^{242,243}$ which provides insight in regard to descending neural drive during maximal voluntary effort. ${ }^{244}$ The V-wave can be essentially detected following the disappearance of the H-reflex during volitional muscle contraction, as descending cortical stimuli supersede the antidromic action potentials of the muscle response. The combined measures of $\mathrm{H}$-reflex and $\mathrm{V}$-wave may provide a more comprehensive understanding of neural adaptations following injury and intervention. ${ }^{245}$

The V-wave is consistently reported within the literature as a proportion of the maximal muscle response $\left(\mathrm{M}_{\max }\right) \cdot{ }^{244-249}$ This relationship is thought to represent the level of efferent $\alpha \mathrm{MN}$ output (neural drive), or ability to volitionally activate a proportion of the entire $\alpha \mathrm{MN}$ pool, while also reflecting reflex excitability. ${ }^{249}$ Changes in M-wave peak-to-peak amplitude have been reported during volitional activation, changes in the length-tension relationship, and contraction mode. ${ }^{244,250}$ For this reason, authors have also reported V-wave measurements relative to the Mwave obtained via supramaximal exogenous electrical stimulus during maximal voluntary contraction ( $\left.\mathrm{M}_{\text {sup }}\right)$, while standardizing type of contraction and joint angles. ${ }^{244,250}$ Adding to the complexity of obtaining accurate data with this measurement is the ability to stabilize the lower limb during MVIC. For this reason, researchers have employed novel approaches, with the subject seated in a semi-reclined, supine position. ${ }^{249}$ However, these approaches may interfere with testing procedures, namely the stimulating electrode. To obtain accurate data, it becomes important to develop a protocol in which an MVIC can be obtained without interfering with testing procedures. 
Current research efforts have utilized the $\mathrm{V}: \mathrm{M}_{\text {sup }}$ ratio as an outcome measure in the evaluation endurance training, ${ }^{245}$ resistance training programs, ${ }^{245,251}$ and neuromuscular electrical stimulation $^{252}$ in the ankle plantar flexors. Inclusion of the motor evoked V-wave represents an interesting compliment to the H-reflex in that it reflects neural changes at the spinal and supraspinal levels. ${ }^{245}$ Understanding and quantifying neuromuscular modulation at the spinal and supraspinal are useful measurements in assessing neuromuscular alterations following joint injury. As measurements are oftentimes repeated over time in intervention studies, it is necessary to demonstrate high reliability. To this point, there is only limited evidence demonstrating the test-retest reliability of the V:M or V: $\mathrm{M}_{\text {sup }}$ in the soleus $(\mathrm{ICC}=0.86){ }^{249}$

\section{Nerve Conduction}

Nerve conduction studies have been utilized in sports medicine practice to evaluate pain, sensation, and motor capabilities following injury. Nerve conduction measures are used as diagnostic tools used to examine the electrical functioning of peripheral nerves. ${ }^{253}$ Additionally, these measures can provide information about the relationship with sensorimotor capacities that influence mobility. Nerve conduction studies are performed by placing electrodes percutaneously to directly stimulate peripheral nerves with an electrical stimulus. From this electrical stimulus, information regarding the path and strength of the resultant afferent action potential can be gained. ${ }^{253}$ Conventional nerve conduction measures include velocity, latency, amplitude, and duration. Conduction velocity describes the speed of an impulse along its axon upon stimulation, which represents how well an electrical impulse is conducted. Latency refers to the time from stimulation of a nerve to the beginning of depolarization. The amplitude of an action potential is the sum of all amplitudes from individual action potentials following a stimulus, with greater amplitude signifying a stronger response. ${ }^{253}$ From these measurements, clinicians can gain a comprehensive assessment of the action potential morphology, and identify sensorimotor impairments. 
Impairments in peripheral nerve conduction measurements have been observed following lateral ankle sprains ${ }^{254-256}$, and knee joint injuries. Additionally, traditional therapeutic modalities, such as cryotherapy, have been reported to impact nerve conduction properties via changes in skin temperature. Understanding how peripheral nerve characteristics can be altered may aid clinicians in the assessment and treatment of individuals following injury.

\section{Estimates of Corticospinal Excitability}

\section{Transcranial Magnetic Stimulation (TMS)}

TMS is a non-invasive tool used to measure neural conduction and processing time, activation thresholds, facilitation and inhibition in the primary motor cortex, and neural connections. ${ }^{22}$ Since its original description in 1985 , single pulse TMS has been widely used to study motor, visual, and somatosensory systems, as well as sensorimotor integration and cognition in patients with a variety of diagnosed disease processes. ${ }^{22}$ It has since emerged in sports medicine research as an intervention and assessment tool primarily in the upper extremity. ${ }^{257}$ Several authors have used TMS to measure cortical excitability in the lower extremities of varied cohorts. ${ }^{24,87,90,229,258}$

Although afferent signals project to the spinal cord directly, joint afferents are known to have extensive supraspinal projections to the cerebral cortex as well. ${ }^{86}$ Supraspinal influence on descending cortical output following injury is often neglected within the context of musculoskeletal research, specifically of the lower extremity, and has only begun to be better understood over the last decade. Currently, the best way to measure neuromuscular changes at the supraspinal level is to assess cortical excitability, which is defined as excitability of the portion of the cerebral cortex responsible for initiating motor commands to skeletal muscle. ${ }^{22}$

Transcranial magnetic stimulation (TMS) provides a method of assessing excitability of the pre-motor area of the cerebral cortex. ${ }^{22}$ TMS produces a small, but powerful field of magnetic energy that depolarizes neural tissue to initiate action potentials. ${ }^{22}$ When a TMS device is placed over the scalp, superficial to the pre-motor area, action potentials are conveyed to the associated 
skeletal muscles resulting in a motor evoked potential (MEP). By stimulating the cortical neurons corresponding to quadriceps activity in the contralateral primary motor cortex, a motor evoked action potential can be detected via surface electromyography. ${ }^{259,260}$ When stimulated during minimal volitional activity of the involved musculature, this measurement is termed active motor threshold (AMT), and has been used as a primary indicator of corticospinal excitability in individuals following knee joint injury. ${ }^{88}$ In sports medicine research, the amplitude and transmission time of MEP's have been used to detect subtle neuromuscular deficiencies in patients with mild traumatic brain injury ${ }^{261}$, patients with ankle ${ }^{262}$ and knee ${ }^{90}$ joint injury, and in individuals who are fatigued due to continuous exercise. ${ }^{89}$

Although TMS protocols widely vary in the literature with respect to coil type and placement, measures of interest, and technique to obtain measurements, acceptable levels of reliability have been established. Reliability of TMS measures has primarily been established in the upper extremities ${ }^{263}$; however more recent researchers have begun to look at the hip ${ }^{258}$, knee, and ankle ${ }^{264}$ musculature with success.

In contrast to assessment technique requiring an electrical stimulus, TMS relies on a magnetic pulse of energy, thereby reducing patient discomfort during testing. Additionally, this technique may provide unique information regarding the influence of descending neural drive to peripheral musculature following injury, not achieved by other techniques. Understanding sensorimotor adaptations at supraspinal centers may allow for a more comprehensive assessment approach, fostering early intervention strategies. 


\section{CONCLUSION}

Sensorimotor deficits occur after joint injury due to alterations in transmission of neural signal to the central nervous system. Lasting impairments likely manifest from a combination of peripheral, spinal, and supraspinal centers. As sensorimotor impairments and resulting neuromuscular deficits are the result of disruption to a multifaceted biological system, comprehensive assessment techniques appear to be warranted. Identifying impairments will provide information to develop optimal strategies for early treatment and active prevention of poor outcomes in active individuals who suffer joint injuries. Future research should use such an approach to identify changes over time in and effort to maximize patient care. Clinical and laboratory based assessment tools can be used in concert to provide clinicians with a comprehensive understanding of sensorimotor impairments following lower extremity joint injury. 
APPENDIX C

\section{Additional Methods}

Table C1. Informed Consent form

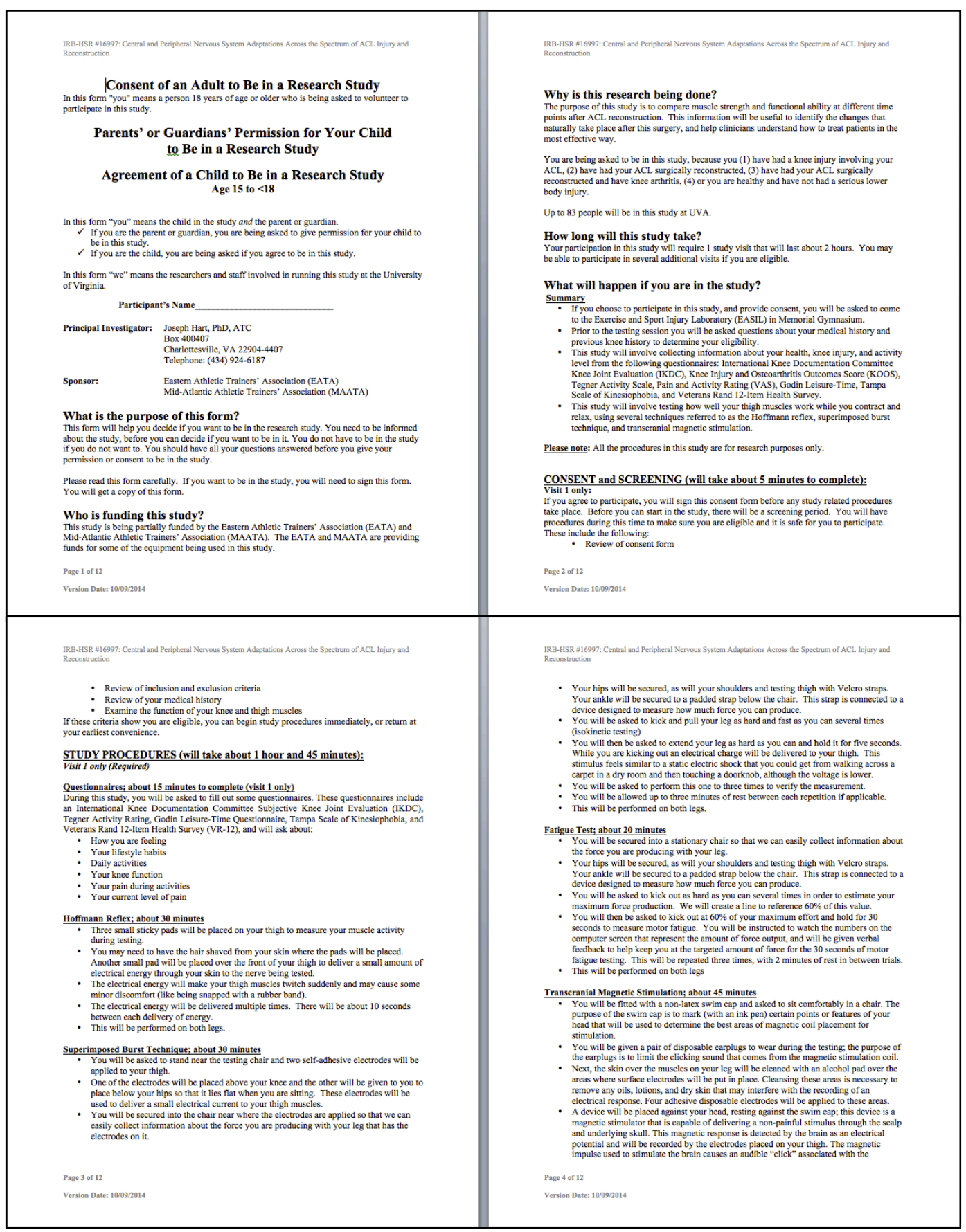


IRB-HSR 216997: Central and Peripheral Nevvous System Adaptations Across the Spectrum of ACL. Injury and
Reconstruction magnetic stimulating coil, and a brief muscle contraction (similar to a muscle "twitch") in
the muscles of your thigh or leg, which will feel like what is felt during standard medical

There is a minimal risk for obtaining a mild headache following the application of the TMS, if such event occurs, it can be cured by taking over the counter headache -

Several measurements will be taken while you are seated. The intensity of the magnent pulse will be gradually increased until a maximum electrical response is detected and recorded. You will not experience any discomfort despite the increased intensity of the stimulation. Once the best response is detected and recorded, a mark will be made on the
swim cap using an ink pen. These markings will be used as reference points for future test sessions. Once these measurements are recorded, you may remove the swim cap

(which will be saved for your subsequent testing sessions.

Visit 2 (Non-required). Those who have an ACL injury, but have not had surgery will not be eligible for the hop tests due to safety considerations

Hop Tests; about 10 minu

he asked to lie down on a treatment table so that the length of your leg can be

- You will then be asked to hop as far as you can on each leg multiple times in different

- directions. The distance you hop will be measured along a tape measure.

Ond will be given I practice hop trials in order to practiong
This will be performed on both legs.

Gait Analysis; about 30 minutes

upright with your shoes and socks off.

- Your skin will be cleaned, similar to the EMG, where each of the sensors will be placed

- En your legs and back.

- You will be given a pair of shoes to put on for testing. If we do not have a pair that fits,

- You will be allowed to use the shoes you are wearing.

5-10 times

- You will be asked to crouch down $5-10$ times with both legs and with one leg at a time

- You will be asked to crouch down and jump straight up 5-10 times with both legs and

will be given time to rest between each task

Page 5 of 12

Version Date: 10/09/2014
IRB-HSR :16997; Central and Peripheral Nevous System Adaptations Across the Spectrum of ACL. Injury and

Follow Up Visits

You may be eligible to return for additional visits every 6 months if enrolled in this study before you are 2 years from the time of your knee surgery. No additional study visits will be available
once you are 2 years from the time of your surgery. If you return for additional study visis, the

If you want to know about the results before the study is done:
During the study you are having an investigational test done. The purpose of the test is not to diagnose any disease or abnormality you may have. Because the test is investigational there is
no way for the study leader to understand if the results are "normal" or "abnormal". However, any test results are concerning, your study leader will let you know.

In addition, as the research moves forward, your study leader will keep you informed of any new you want to continue in the study. The final the information from everyone is combined and reviewed. At that time you can ask for more

What are the risks of being in this study?

Risks and side effects related to the study procedures include:

$\frac{\text { Likely }}{\text {. }}$

Less Likely

- You could experience minor, short - lasting skin irritation where the self-adhesive
electrodes have been placed

electrodes have been placed.
Risk of temporary discomfort from electrical stimulation during superimposed burst an

he transcranial magnetic

Rare but serious

aree a seizure if you have a history of epilepsy or other seizure disorder.

Other unexpected risks:

You may have side effects that we do not expect or know to watch for now. Call the study

Pase 6 of 12

Version Date: 10/1092014

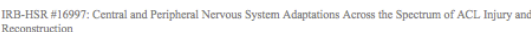

Could you be helped by being in this study? Health Informantios no benefif to individuals. It poses minimal risk of breach of Protected regulation

What are your other choices if you do not join this study? You do not have to be in this study to be treated for your illness or condition. You can get the
usual treatment even if you choose not to be in this study. The usual treatment would include physical therapy as prescribed by your treating physician. If you are an employee of UVa your job will not be affected if you decide not to participate in this study.
If you are a student at UVa, your grades will not be affected if you decide not to participate in
this study.

Will you be paid for being in this study?

If you have had an injury to your ACL, you will be paid $\$ 20$ for finishing this study.
You should get your payment about $4-6$ weeks after finishing the study by check. The income may be reported to the IRS as incornent

If you owe money to any Virginia state agency, the state can use the money you earn in this study to pay those debts. These state agencies include the UVa Medical Center, $\mathrm{VCU}$ Medical
Center or a college or university. The money may be withheld to pay back debt for such things Center or a college or university. The money may be withheld to pay back debt for such things
as unpaid medical bills, taxes, fines, child support. Even if this happens, the money you earn may be reported to the IRS as taxable income.

For healthy subjects:

Will being in this study cost you any money?

Allil the procedures in this study will be provided at no cost to you or your health insurance. You
will be responsible for the cost of travel to come to any study visit and for any parking costs.

What if you are hurt in this study?

If you are hurt as a result of being in this study, there are no plans to pay you for medical
expenses, lost wages, disability, or discomfort. The charges for any medical treatment you

receive will be billed to your insurance. You will be responsible for any amount your insurance

does not cover. Yo
by signing this form.

What happens if you leave the study early?

Page 7 of 12

Version Date: 10/09/2014
IRB-HSR 116977: Central and Peripheral Nervous System Adaptations Across the Spectrum of ACL Injury and
Reconstruction

You can change your mind about being in the study any time. You can agree to be in the study now and change your mind later. If you decide to stop, piease tell us right away. Yo
to be in this study to get services you can normally get at the University of Virgnia

Even if you do not change your mind, the study leader, Dr. Joe Hart, can take you out of the

a). Some of the reasons for doing so may include
a) Your study physician is concerned about your healtr

b) Your disease gets wo

atment are too dangerous for $y$ o

on shows the treatment will not work or is not safe for you

e) You do not follow your doctor's instructions
f) The study sponsor closes the study for safety, administrative or other reasons

If you decide to stop being in the study, we will ask you to please notify Dr. Joe Hart in writing
at 210 Emmet Street South, P.O. Box 400407, Charlottesville, VA 22904-4407. Please note that any information already obtained from you may continue to be used by the investigators of this

How will your personal information be shared?

The UVa researchers are asking for your permission to gather, use and share information about
you for this study. If you decide not to give your permission, you cannot be in this study, but you for this study. If you decide not to give your permission
you can continue to receive regular medical care at UVA.

If you sign this form, we may collect any or all of the following information

about you:
: Personal information such as name, address and date of birth
OScial Security number ONLY IF you are being paid to be in

Social Security number ONLY IF you are being paid to be in this study medical records and test results from before, during and after the study from any of your

Who will see your private information?

- The researchers to make sure they can conduct the study the right way, observe the effects of the study and understand its results

People or groups that overssee the study to make sure it is done correctly

Tax reporting offices (if you are paid for being in the study)

People who evaluate study results, which can include sponsors and other companies that make the drug or device being studied, researchers at other sites conducting the same study, $(\mathrm{FDA})$ if the study is regulated by the FDA. 
IRB-HRR \#16097: Central and Peripheral Nervous System Aduptations Accoss she Spectrum of ACL Injury ant
Reconstruction

Some of the people outside of UVa who will see your information may not have to follow the same privacy laws that we follow. We ask them to protect your privacy. However, they may
release your information to others, and it may no longer be protected by those laws.

The information collected from you might be published in a medical journal. This would be The information collected from you might be published in a medical journal. This would be
done in a way that protects your privacy. No one will be able to find out from the article that you
were in the study.

What if you sign the form but then decide you don't want your private 政 cancel it, please send a letter to the researchers listed on this form. Then you will no longer be the study. The research

Please contact the researchers listed below to:

- Obtain more information about the study

Ask a question about the study procedures or treatments
Report an illness, injury, or other problem (you may also need to tell your regular doctors)

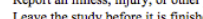

Express a concern about the study

Joe Hart, PhD, ATC

Human Services, Curry School of Education

Charlottesville, VA 22904-4407
Telephone: (434) 924-6187

What if you have a concern about a study?

You may also repor a concern about a study or ask questions about your rights as a research
subject by contacting the Institutional Review Board listed below.

University of Virginia Institutional Review Board for Health Sciences Research

PO Box 800483

Charlottesville, Virginia 22908
Telephone: $434-924-9634$

When you call or write about a concern, please give as much information as you can. Include the
name of the study leader, the IRB-HSR Number (at the top of this form) and details about the name of the study leader, the IRB-HSR Number (at the top of this form), and details about the problem. This will help officials 1 cok
have to give your name.

Page 9 of 12

version Date: 10/09/2014

B-HSR \#16997: Central and Peripheral Nevvous System Adaptation Acesese me Spectrum of ACL. Injury of

Signatures

What does your signature mean?
Before you sign this form, please ask questions about any part of this study that is not clear to theve been receive a copy of this signed document

Consent From Adult

PARTICIPANT $\quad \overline{\text { PARTICIPANT }}$ (PRINT)
To be completed by participant if 18 years of age or older.

Consent From Impartial Witness process and sign the following statement. The subject may place an $X$ on the Participant

Signature line above.

agree the information in this informed consent form was presented orally in my presence to the subject and the subject had the opportunity to ask any questions he/she had about the study.
also agree that the subject freely gave their informed consent to participate in this trial.

$\begin{array}{lll}\substack{\text { IMPARTIAL WITNESS } \\ \text { (SIGNATURE) }} & \begin{array}{l}\text { IMPARTIAL WITNESS } \\ \text { (PRINT) }\end{array}\end{array}$

Person Obtaining Consen By signing below you confirm that you have fully explained this study to the potential subject,
allowed them time to read the consent or have the consent read to them, and have answered all
their ruestions

$\begin{array}{ll}\text { PERSON OBTAINING CONSENT } & \\ \text { (SIGNATURE) } & \text { PERSON OBTAINING } \\ \text { CONSENT (PRINT) } & \text { DATE }\end{array}$

IRB-HSR \#16997: Central and Peripheral Nervous System Adappations Across the Spectrum of ACL Injury and

Parental/Guardian Permission

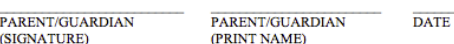

Consent From Impartial Witnes insenting process and sign the following statement. The parent may place an $\mathbf{X}$ on the

I agree the information in this informed consent form was presented orally in $m y$ presence to the

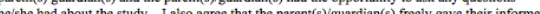
consent for their child to participate in this trial

$\begin{array}{lll}\begin{array}{l}\text { IMPARTIAL WITNESS } \\ \text { (SIGNATURE) }\end{array} & \begin{array}{l}\text { MPAARTIAL WITNESS } \\ \text { (PRINT) }\end{array} & \text { DATE } \\ & & \end{array}$

Person Obtaining Parenta/Guardian Permission

By signing below you confirm that you have fully explained this study to the parent guardian allowed them time to read the consent or have the consent read to them, and have answered all

PERSON OBTAINING PARENTAL

GUARDIAN PERMISSIO
(SIGNATURE)

PERSON OBTAINING

PARENTAL/GUARDIA

$\overline{\text { DATE }}$ (PRINT NAME) If this consent form is read to the parent(s) because the parent(s) is blind or ilititerate, an

Assent from Child
Consent from the parentguardian MUST be obtained before approaching the child for their assent.

$\begin{array}{lll}\begin{array}{l}\text { PARTICIPANT } \\ \text { (SIGNATURE) }\end{array} & \overline{\text { PARTICIPANT }} \\ \text { (PRINT) } & & \\ \text { DATE } & \end{array}$

Person Obtaining Assent of the Child (less than 18 years of age) Consent from the parent/guardian MUST be obtained before approaching the child for their assent.

By signing below you confirm that the study has been explained to the child (less than 18 years

$\overline{\text { PERSON OBTAINING }}$ PERSON OBTAINING $\begin{array}{ll}\begin{array}{l}\text { ASSENT } \\ \text { (SIGNATURE) }\end{array} & \begin{array}{l}\text { ASSENT } \\ \text { (PRINT) }\end{array} \\ & \end{array}$ 
Table C2. Transcranial Magnetic Stimulation Screening Form

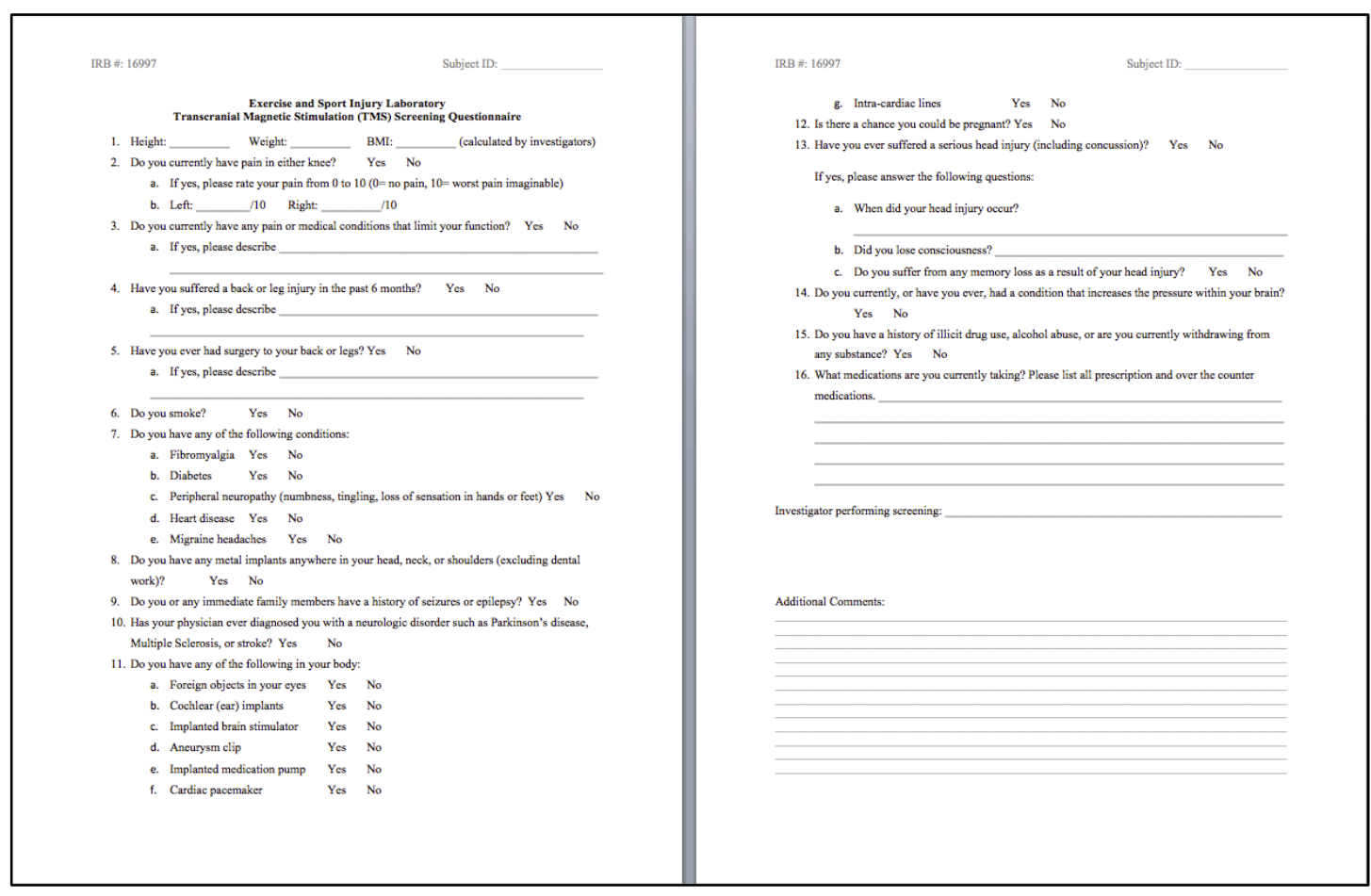


Table C3. International Knee Documentation Committee (IKDC) Subjective Knee Evaluation

\begin{tabular}{|c|c|}
\hline 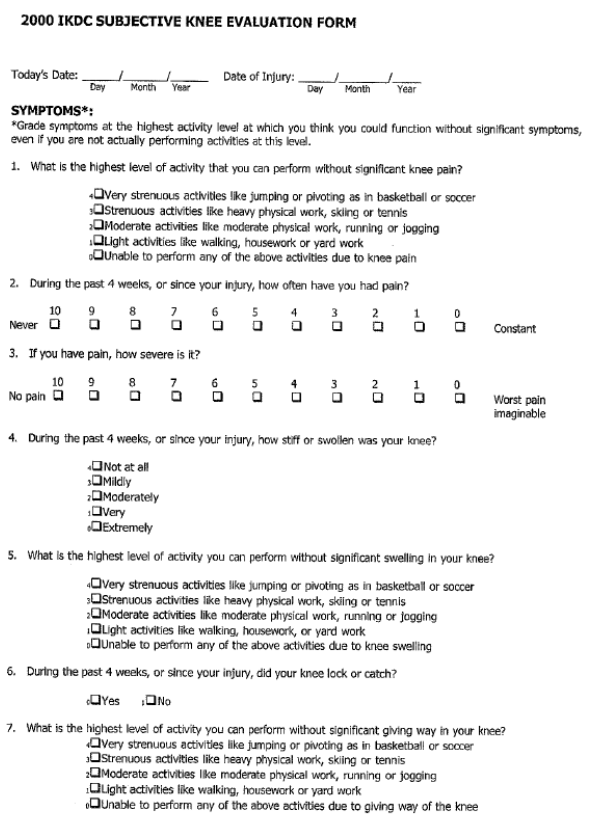 & 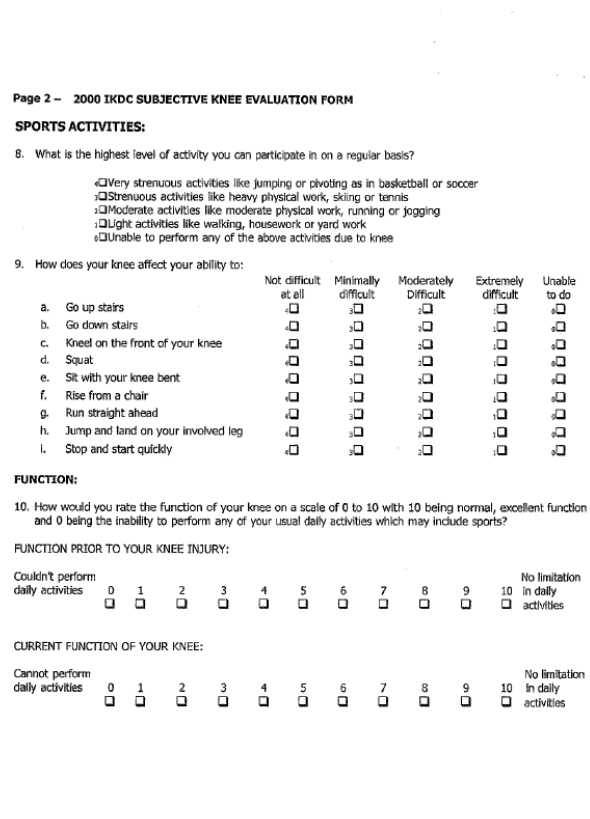 \\
\hline
\end{tabular}


Table C4. Knee Injury and Osteoarthritis Outcome Score (KOOS)

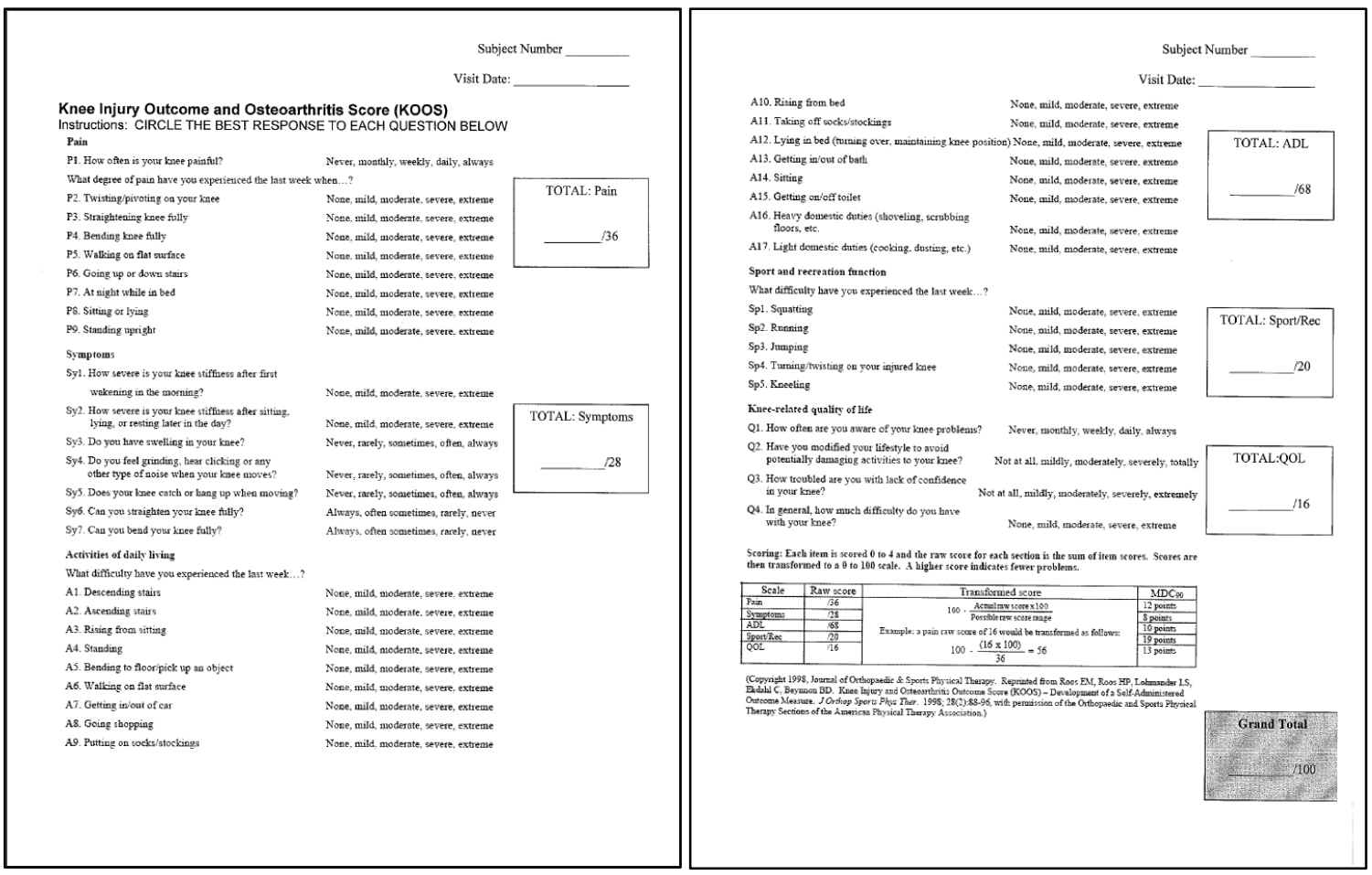


Table C5. Visual Analog Scale (VAS) for Pain at Rest

\section{Pain and Activity Rating}

Place a vertical mark or ' $X$ ' on the line below that best represents the level of pain you are experiencing now.

Right Side

No Pain

Worst possible,
unbegrable,

unbearable,
excruciating pain

Rating:

excruciating pain

Left side

No Pain

Worst possible,

unbearable,

excruciating pain

Rating: _ $\quad \mathrm{cm}$ 
Table C6. Tegner Activity Level Scale

\section{TEGNER ACTIVITY SCALE}

Please indicate in the spaces below the HIGHEST level of activity that you participated in BEFORE YOUR.MJURY and the highest level you are able to participate in CURRENTLY

\begin{tabular}{|ll}
\hline BEFORE INJURY: Level___ CURRENT: Level____ _ C \\
\hline
\end{tabular}

\begin{tabular}{|c|c|}
\hline Level 10 & Competitive aporis-socoer, football, nugby (national elike) \\
\hline Level 9 & Compeitive sports- aocoer, footbell, rugby (lower divișions), ice hockey, wrestling, gymnastics, baskethall \\
\hline Level 8 & $\begin{array}{l}\text { Compatitive sports- racquethall or bandy, squash or badminton, track and feld athletics (jumping. etc.), down- } \\
\text { hill sking }\end{array}$ \\
\hline Level 7 & $\begin{array}{l}\text { Competitive sports- tennis, running, motcrcers speedway, handbell } \\
\text { Recreational sports-soccer, footbsil, rugby, bandy, ice hockey, basketball, squash, racquetball, running }\end{array}$ \\
\hline Leves 6 & $\begin{array}{l}\text { Recreational sports- tennis and badminton, handball, racquetball, dCWn-hill skiling. jogging at lasest } 5 \text { times per } \\
\text { woek }\end{array}$ \\
\hline Lovel 5 & $\begin{array}{l}\text { Work- heavy labor (construction, etc.) } \\
\text { Competitive sports-cycling, cross-country sking, } \\
\text { Recreational sports- jogging on uneven ground at least twice weakhy }\end{array}$ \\
\hline Level 4 & Work- moderately heavy laber $(e, g$, truck driving, otc. $\}$ \\
\hline Lewal 3 & Work- light laber (nursing, etc) \\
\hline Level 2 & $\begin{array}{l}\text { Work- light labor } \\
\text { Walking on uneven ground possible, but impossible to back pack or hike }\end{array}$ \\
\hline Level 1 & Work- sedentary (eecretarial, ete) \\
\hline Leval 0 & Sick leave or disability pension because of knee problems \\
\hline
\end{tabular}

All information in this Worksheet is Confidential. 
Table C7. Godin Leisure-Time Exercise Questionnaire

\section{Godin Leisure-Time Exercise Questionnaire}

1. During a typical 7-Day period (a week), how many times on the average do you do the following kinds of exercise for more than 15 minutes during your free time (write on each line the appropriate number).

a) STRENUOUS EXERCISE (HEART BEATS RAPIDLY)

(e.g. running, jogging, hockey, football, soccer, squash, basketball, cross country skiing, judo, roller skating, vigorous swimming,

vigorous long distance bicycling)

b) MODERATE EXERCISE (NOT EXHAUSTING)

(e.g., fast walking, baseball, tennis, easy bicycling, volleyball, badminton, easy swimming, alpine skiing, popular and folk dancing)

c) MILD EXERCISE (MINIMAL EFFORT)

(e.g., yoga, archery, fishing from river bank, bowling, horseshoes, golf, snow-mobiling, easy walking)

Times Per Week

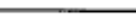


Table C8. Tampa Scale for Kinesiophobia (TSK)

TAMPA SCALE FOR KINESIOPHOBIA

(MILLER, KORI AND TODD 1991)

\begin{tabular}{|c|c|c|c|c|c|}
\hline & $\begin{array}{l}\text { CIRCLE THE NUMBER THAT BEST DESCRIBES YOUR } \\
\text { BELIEF FOR EACH STATEMENT BELOW: }\end{array}$ & 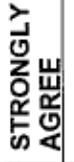 & 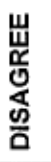 & $\begin{array}{l}\text { 㟧 } \\
\frac{1}{0} \\
\frac{1}{4}\end{array}$ & 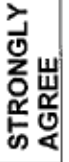 \\
\hline 1 & I'm afraid that I might injury myself if I exercise & 1 & 2 & 3 & 4 \\
\hline 2 & If I were to try to overcome it, my pain would increase & 1 & 2 & 3 & 4 \\
\hline 3 & My body is telling me I have something dangerously wrong & 1 & 2 & 3 & 4 \\
\hline 4 & My pain would probably be relieved if I were to exercise & 1 & 2 & 3 & 4 \\
\hline 5 & People aren't taking my medical condition seriously enough & 1 & 2 & 3 & 4 \\
\hline 6 & My accident has put my body at risk for the rest of my life & 1 & 2 & 3 & 4 \\
\hline 7 & Pain always means I have injured my body & 1 & 2 & 3 & 4 \\
\hline 8 & $\begin{array}{l}\text { Just because something aggravates my pain does not mean } \\
\text { it is dangerous }\end{array}$ & 1 & 2 & 3 & 4 \\
\hline 9 & I am afraid that I might injure myself accidentally & 1 & 2 & 3 & 4 \\
\hline 10 & $\begin{array}{l}\text { Simply being careful that I do not make any unnecessary } \\
\text { movements is the safest thing I can do to prevent my pain from } \\
\text { worsening }\end{array}$ & 1 & 2 & 3 & 4 \\
\hline 11 & $\begin{array}{l}\text { I wouldn't have this much pain if there weren't something } \\
\text { potentially dangerous going on in my body }\end{array}$ & 1 & 2 & 3 & 4 \\
\hline 12 & $\begin{array}{l}\text { Although my condition is painful, I would be better off if I were } \\
\text { physically active }\end{array}$ & 1 & 2 & 3 & 4 \\
\hline 13 & $\begin{array}{l}\text { Pain lets me know when to stop exercising so that I don't } \\
\text { injure myself }\end{array}$ & 1 & 2 & 3 & 4 \\
\hline 14 & $\begin{array}{l}\text { It's really not safe for a person with a condition like mine to be } \\
\text { physically active }\end{array}$ & 1 & 2 & 3 & 4 \\
\hline 15 & $\begin{array}{l}\text { can't do all the things normal people do because it's too easy } \\
\text { for me to get injured }\end{array}$ & 1 & 2 & 3 & 4 \\
\hline 16 & $\begin{array}{l}\text { Even though something is causing me a lot of pain, I don't think } \\
\text { it's actually dangerous }\end{array}$ & 1 & 2 & 3 & 4 \\
\hline 17 & No one should have to exercise when he/she is in pain & 1 & 2 & 3 & 4 \\
\hline
\end{tabular}

Reprinied from:

Pain, Fear of movement/(re) injury in chronic low back pain and its relation to behavioral pefformance, 62, Vlaeven, J., Kole-Snịders A, Boeren

R., van Eek H., 371. Cocyright (1995) with permission from International Association for the Study of Pain.

Alexander C. Simotas, M.D. Assistant Professor of Rehabijitation Medicine The Hospital for Special Surgery - 429 East $75^{\text {th }}$ Street, $4^{\text {th }}$ Floor - New York, NY 10021

Tel: $212.606 .1879 \cdot$ Fax: 212.794 .192 
Table C9. The Veterans Rand 12-Item Health Survey (VR-12)
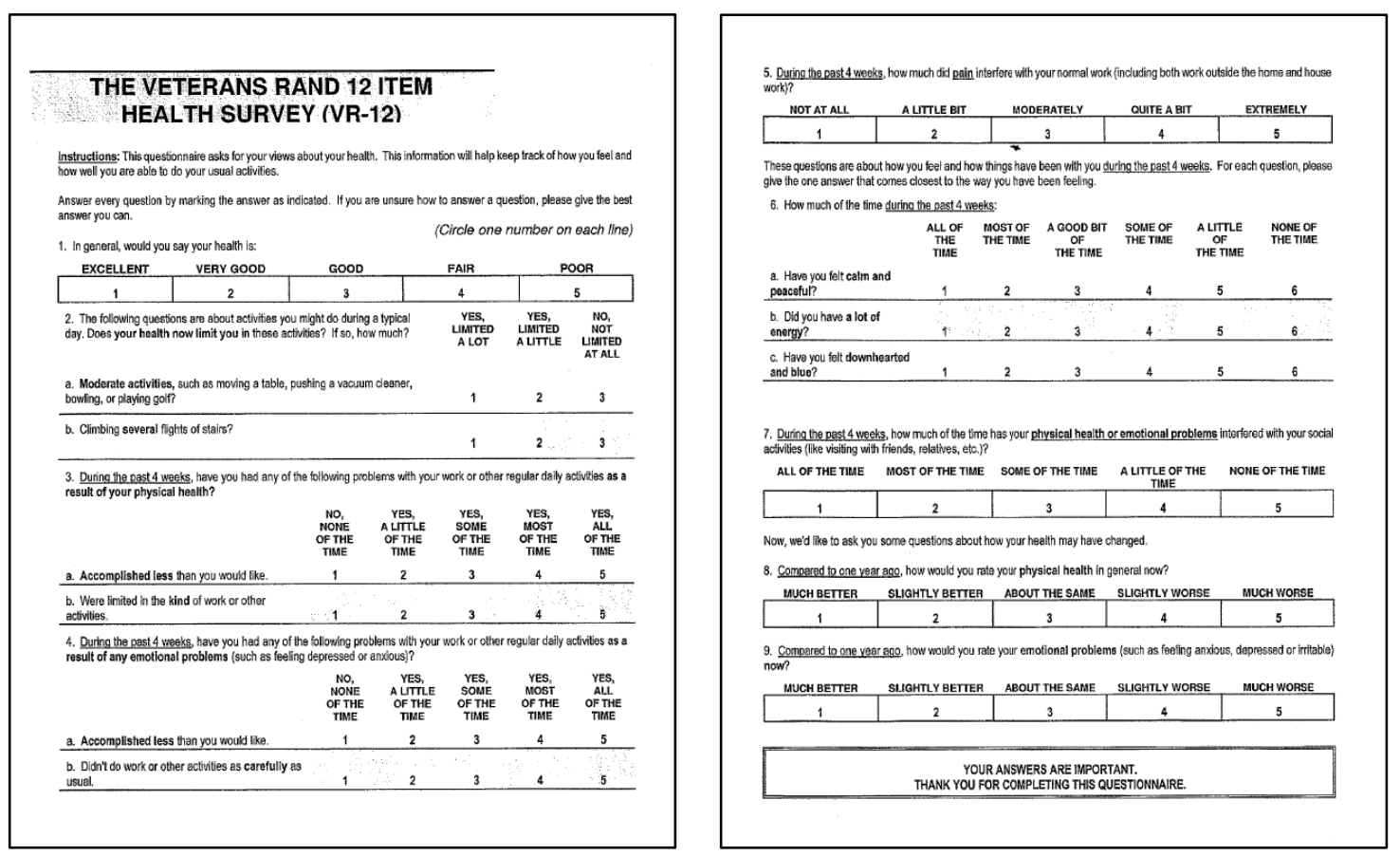


\section{Table C10. Overall Study Procedures}

1. Attend study visit at Memorial Gymnasium (\#224B)

a. Obtain informed consent (Table C1)

b. Complete screening and intake form (Table C2)

i. Assess eligibility criteria

ii. Obtain subject body mass $(\mathrm{kg})$

iii. Obtain subject height $(\mathrm{cm})$

iv. Determine limb dominance - "leg used to kick a ball"

v. Determine involved (injured) limb in ACL subjects

vi. Determine involved (non-dominant) limb in healthy subjects

vii. Determine order of limb testing - counterbalanced per group

c. Complete patient reported outcome measures (Tables C3-C9)

d. Assess quadriceps Hoffmann reflex ${ }^{\mathrm{a}}$ (Table C12)

e. Assess quadriceps isokinetic torque ${ }^{\mathrm{b}}$ (Table C13)

f. Assess quadriceps MVIC torque and central activation ratio ${ }^{b}$ (Table C14)

g. Assess quadriceps fatigue index ${ }^{\mathrm{b}}$ (Table $\left.\mathrm{C} 15\right)$

h. Assess quadriceps active motor threshold (Table C16)

i. ${ }^{a}$ Study procedure(s) repeated in the contralateral limb immediately following completion of the starting limb

j. $\quad{ }^{b}$ Study procedure(s) repeated in the contralateral limb after each test has been completed in the starting limb

k. Dismiss subject from the study 
Figure C1. Study Flowchart

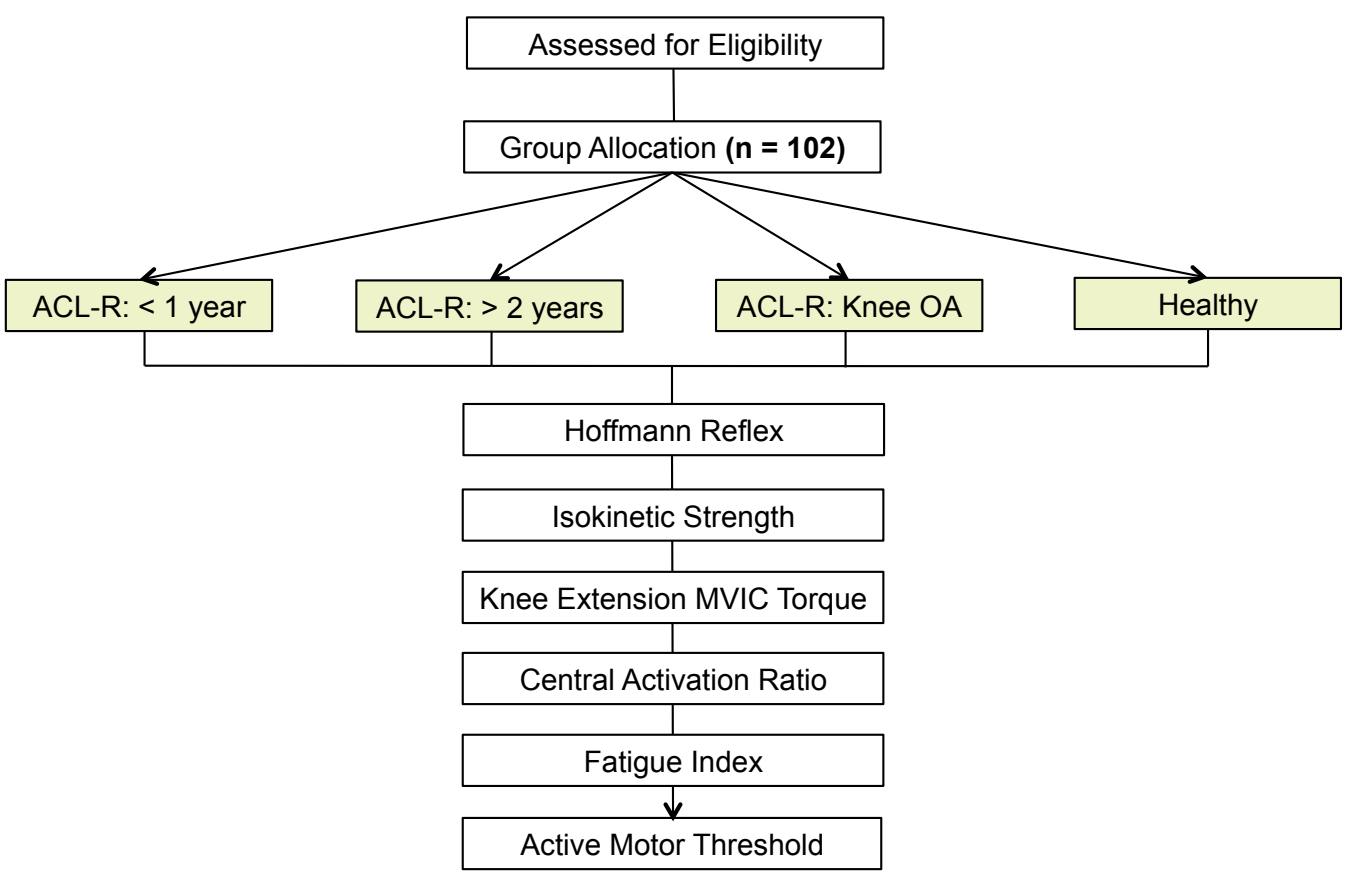

Figure $\mathrm{C} 1$. Flow chart of study procedures. Each measure was recorded bilaterally, with limb counterbalanced within each group. 


\section{Table C11. Patient Reported Outcome Measures}

1. Subjects will complete subjective measures in a quiet room under supervision once determined to be eligible

2. All subjects will complete 7 subjective measures:

a. International Knee Documentation Committee (IKDC) Subjective Knee Evaluation Form (Table C3)

b. Knee Injury and Osteoarthritis Outcome Score (KOOS) (Table C4)

c. Visual Analog Scale (VAS) for Pain at Rest (Table C5)

d. Tegner Activity Level Scale (Table C6)

e. Godin Leisure-Time Exercise Questionnaire (Table C7)

f. Tampa Scale for Kinesiophobia (TSK) (Table C8)

g. Veterans Rand 12-Item Health Survey (VR-12) (Table C9) 


\section{Table C12. Quadriceps Hoffmann Reflex Setup and Procedures}

1. Biopac Setup

a. Connect UIM100C, STM100C, and EMG100C to the MP150 unit

b. Connect STMISOC to the STM100C via output jack

c. Connect MP150 to the computer using a LAN wire

d. Turn on MP150 unit and the computer

e. STM100C Settings

i. $\quad$ Source $=$ OUT0

ii. Level $=100 \%$

iii. Polarity $=$ POS

iv. Current $=\mathrm{DC}$

f. EMG100C Settings

i. Gain $=1000$

ii. $\quad$ Filter $=$ Off

iii. $\mathrm{LP}=5 \mathrm{kHz}$

iv. $\mathrm{HP}=1.0 \mathrm{~Hz}$

g. STMISOC Settings

i. Voltage Monitor $=0.5 \mathrm{~V}$

ii. Voltage Switch = Voltage (1:10) $200 \mathrm{~V}$ Max

h. Plug active and dispersive electrodes into the STMISOC

2. Acqknowledge Setup

a. Open Acqknowledge 4.2.0 for Windows and select the attached MP150 unit

b. MP150 | Setup Channels | Analog menu

i. Channel 1

1. Sample Rate $=2000 \mathrm{~Hz}$

2. Label $=$ QUAD

3. Check all boxes associated with this channel

ii. Channel 2

1. Sample Rate $=2000 \mathrm{~Hz}$

2. Label $=$ STIM

3. Check all boxes associated with this channel

c. MP150 | Set Up Acquisition

i. Change menus to "Record" and "Append"

ii. Sample Rate $=2000 \mathrm{~Hz}$

iii. Acquisition Length $=79 \mathrm{msec}$

d. MP150 | Set Up Stimulator

i. Click square wave icon

ii. Duration $=$ Output Once

iii. Stimulator Sample Rate $=2000 \mathrm{~Hz}$

iv. Seg \#1 Width $=3.0 \mathrm{msec}$

v. Seg \#2 Width $=1.0 \mathrm{msec}$

vi. Seg \#3 Width $=0.0 \mathrm{msec}$

vii. Seg \#4 Width $=0.0 \mathrm{msec}$

viii. Seg \#5 Width $=33.5 \mathrm{msec}$

e. MP150 | Show Manual Control

i. Analog Outputs: Out $1=0.0$

ii. Analog Outputs: Out $2=10.0$

iii. Open data journal and stimulator window

f. Click start button to confirm proper setup 
3. Subject Preparation (Figure C2)

a. Position the subject supine on the testing table

b. Place small half foam bolster under subjects' knees

c. Identify the bulk of vastus medialis during manually resisted isometric knee extension contraction

i. Shave the area

ii. Debride skin with an abrasive pad or gauze

iii. Clean with isopropyl alcohol

d. Place two surface EMG electrodes in the prepared area

i. Parallel with muscle fiber orientation

ii. Interelectrode distance of $2.0 \mathrm{~cm}$

e. Identify an area on the distal anteromedial tibia for the ground (reference) electrode

i. Shave the area

ii. Debride skin with an abrasive pad or gauze

iii. Clean with isopropyl alcohol

f. Place one surface EMG electrode in the prepared area

g. Attach the leads from the EMG100C unit to the active and ground (reference) electrodes

i. $\quad$ Proximal active electrode $=$ Red lead

ii. Distal active electrode $=$ White lead

iii. Ground (reference) electrode $=$ Black lead

h. Drape the subject

i. Liberally gel the stimulating and dispersive electrodes

j. Palpate the inguinal fold and locate the femoral pulse

k. Move slightly lateral and place the active stimulating electrode over the femoral nerve

1. Place the dispersive electrode on the posterior thigh near the gluteal fold

$\mathrm{m}$. Instruct the subject to remain calm and relaxed, and to close their eyes throughout testing, while keeping their arms at their side

$\mathrm{n}$. Turn off the lights and any additional unnecessary electronics in the testing area

o. Confirm appropriate setup and begin testing procedures

\section{Data Collection (Figure C2)}

a. Confirm that the data window, stimulator window, and data journal are open in the AcqKnowledge software

b. Change the measurement tools within the data window to confirm that P-P and delta $\mathrm{T}$ are available

c. Change the Seg \#2 Level in the stimulator window to 2.0

d. Click the start button in the data window to trigger a stimulus (or CTL + space)

i. Monitor for subject motor response or discomfort throughout testing

e. Progressively increase the stimulus by $0.5 \mathrm{~V}$ until an H-reflex is measured

i. Highlight the EMG response within the data window and measure the P$\mathrm{P}$ amplitude (confirm location of EMG response using delta $\mathrm{T}$ )

ii. Save this value to the data journal

f. Allow at least 10 seconds between stimuli

g. After the first measureable H-reflex, continue to progressively increase stimulation intensity by $0.2 \mathrm{~V}$

h. Continue to change the stimulus intensity by $0.1 \mathrm{~V}$ increments or decrements to identify the maximum P-P EMG measurement 
i. Repeat measurement and recording steps e.i-e.ii above

i. Confirm the maximum recorded H-reflex value and complete 3 trials at the corresponding stimulus intensity

j. After recording maximum H-reflex, progressively increase the stimulus intensity by $0.5 \mathrm{~V}$ until a measurable $\mathrm{M}$ response is present, and a measureable H-reflex is no longer present

k. Record the value for the $\mathrm{M}$ response in the data journal (confirm location of EMG response using delta $\mathrm{T}$ )

1. After the first measureable $\mathrm{M}$ response, continue to progressively increase stimulation intensity by $0.2 \mathrm{~V}$

i. Repeat measurement and recording steps e.i-e.ii above

$\mathrm{m}$. Confirm the maximum recorded $\mathrm{M}$ response value, and complete 3 trials at the corresponding stimulus intensity

n. Repeat procedures on contralateral limb

o. Save the data file and remove the testing equipment from the subject

5. Data Processing

a. Open the data file

b. Transform | Digital Filters | FIR | Bandpass

i. Low Frequency Cutoff $\mid$ Fixed At $\mid 10 \mathrm{~Hz}$

ii. High Frequency Cutoff | Fixed At $\mid 500 \mathrm{~Hz}$

iii. Number of Coefficients $\mid$ Optimize for sample rate and cutoff

iv. Check the box next to Filter entire waveform

c. Transform | Digital Filters |FIR | Bandstop

i. Low Frequency Cutoff | Fixed At $\mid 59.5 \mathrm{~Hz}$

ii. High Frequency Cutoff $\mid$ Fixed At $\mid 60.5 \mathrm{~Hz}$

iii. Number of Coefficients $\mid$ Optimize for sample rate and cutoff

iv. Check the box next to Filter entire waveform

d. Start processing at the first maximum H-reflex trial

e. Highlight the EMG response

f. Measure the P-P amplitude and record in the data journal

i. Repeat this procedure for the 3 recorded maximum trials

g. Locate the 3 maximum M-wave trials

h. Highlight the EMG response

i. Measure the P-P amplitude and record in the data journal

i. Repeat this procedure for the 3 recorded maximum trials

6. Save file 
Figure C2. Quadriceps Hoffmann Reflex
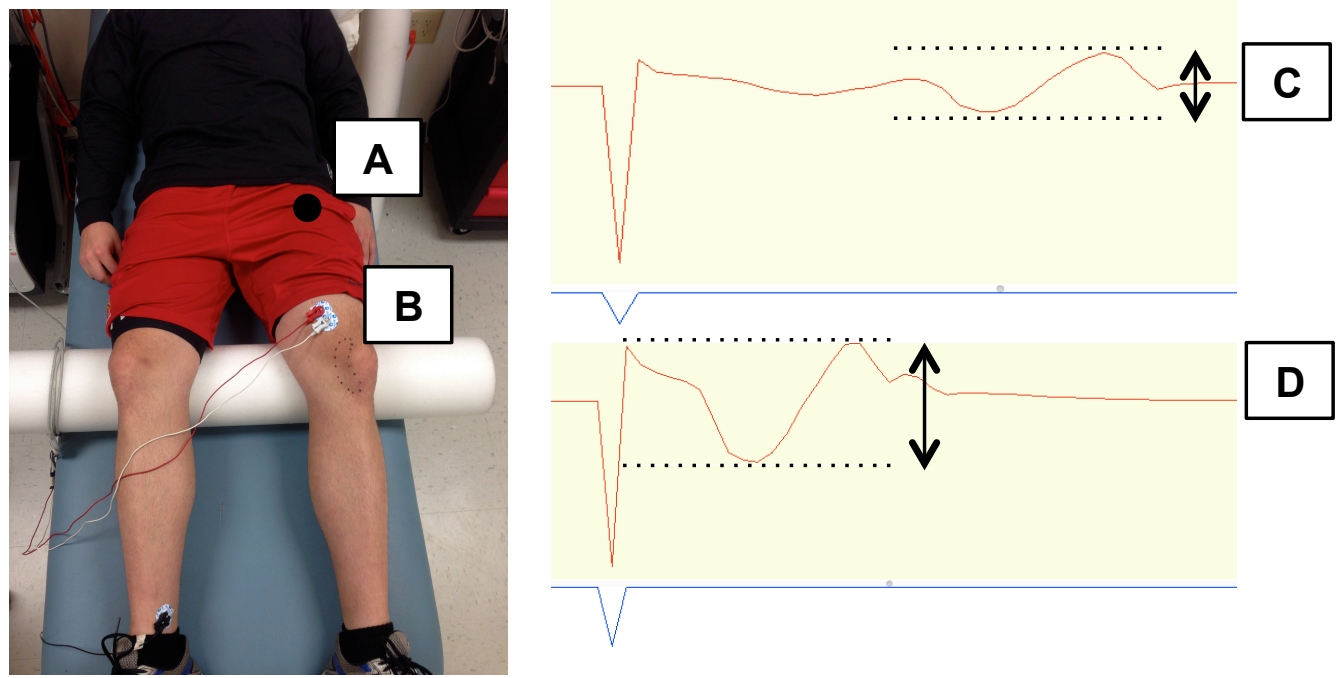

Figure C2. Represents Hoffmann reflex procedures, including (A) stimulating electrode placed over the femoral nerve and (B) recording surface EMG electrodes placed over the vastus medialis. Data were processed by recording the peak-peak amplitude for maximal H-reflex (C) and maximal $\mathrm{M}$ response (D). 


\section{Table C13. Quadriceps Isokinetic Torque Setup and Procedures}

\section{Biodex Setup}

a. Turn on the Biodex System 3

b. Allow time for calibration and attach the desired knee attachment

c. Position the back of the Biodex chair at 80 degrees

d. Confirm the Biodex arm is perpendicular to the floor using a handheld dynamometer

e. Select isokinetic mode and press the start button

f. Press the computer control button

2. Computer Setup

a. Open Biodex System 3 software

b. File | Setup - Confirm simulation mode is turned off

c. Select the "patient" icon

d. Add patient | enter the appropriate demographics

e. Select the "protocol" icon

f. Click protocol definition

g. Select isokinetic unilateral | knee extension/flexion | con/con: test: 90/90, 180/180 (ACL-R Ortho Protocol) | close

h. Select the "range of motion" icon

i. Click on the appropriate side (i.e. left, right)

j. Click define new range of motion / clear

k. Extend subjects' test limb to a neutral position (0 degrees) $\mid$ press black "hold" button on Biodex unit | click "Away" on computer | press black "hold" button

1. Flex subjects' test limb to 70 degrees | press black "hold" button on Biodex unit | click "Toward" on computer | press black "hold" button again | press "continue"

m. Flex subjects' test limb to 90 degrees | press black "hold" button on Biodex unit | click "position" on computer | press black "hold" button again

n. Extend subjects' test limb to a neutral position ( 0 degrees) $\mid$ ask subject to relax the leg | press black "hold" button on Biodex unit | click "limb weight" on computer | press black "hold" button

o. Click the start button to begin testing

3. Subject Preparation (Figure C3)

a. Position the subject in the dynamometer chair in an upright seated posture

i. Knees flexed to 90 degrees

ii. Hips flexed to 80 degrees

iii. Restrain the subject using the lap strap

iv. Engage the ankle strap $2 \mathrm{~cm}$ proximal to the lateral malleolus

b. Provide instructions on proper knee extension testing technique

i. "Sit up straight"

ii. "Do not lift your backside out of the seat"

iii. "Do not rotate or arch your back"

iv. "Do not grip the handles on the dynamometer"

v. "Cross your arms across your chest"

vi. "Concentrate on kicking out and pulling back as hard and as fast as you can using only your thigh muscles" 


\section{Data Collection}

a. Click the start button to begin testing

b. Subject will perform 2-4 practice trials

c. Subject will complete 8 repetitions (full extension + full flexion $=1$ repetition) at 90 degrees/ second

d. Subject will rest for 30 seconds

e. Subject will perform 2-4 practice trials

f. Subject will complete 8 repetitions (full extension + full flexion $=1$ repetition) at 180 degrees/ second

g. Click the continue button on the screen following testing

5. Data Processing

a. Open the data file

b. Select the "report" icon

i. Under "choose options" click on "window isokinetic data" and "use metric units"

ii. Under "choose report" click on "comprehensive evaluation"

iii. Click print preview

c. Select print screen (prtscn) on keyboard

d. Open Microsoft Word

e. Paste $(C T L+V)$ report in Word

i. Repeat for each report: 4 total (involved and uninvolved limb at 90 and 180 degrees/ second)

f. Save Word document with prefix "Isokinetic_"

g. Record quadriceps peak torque, total work, and average power in data spreadsheet 
Figure C3. Isokinetic Torque
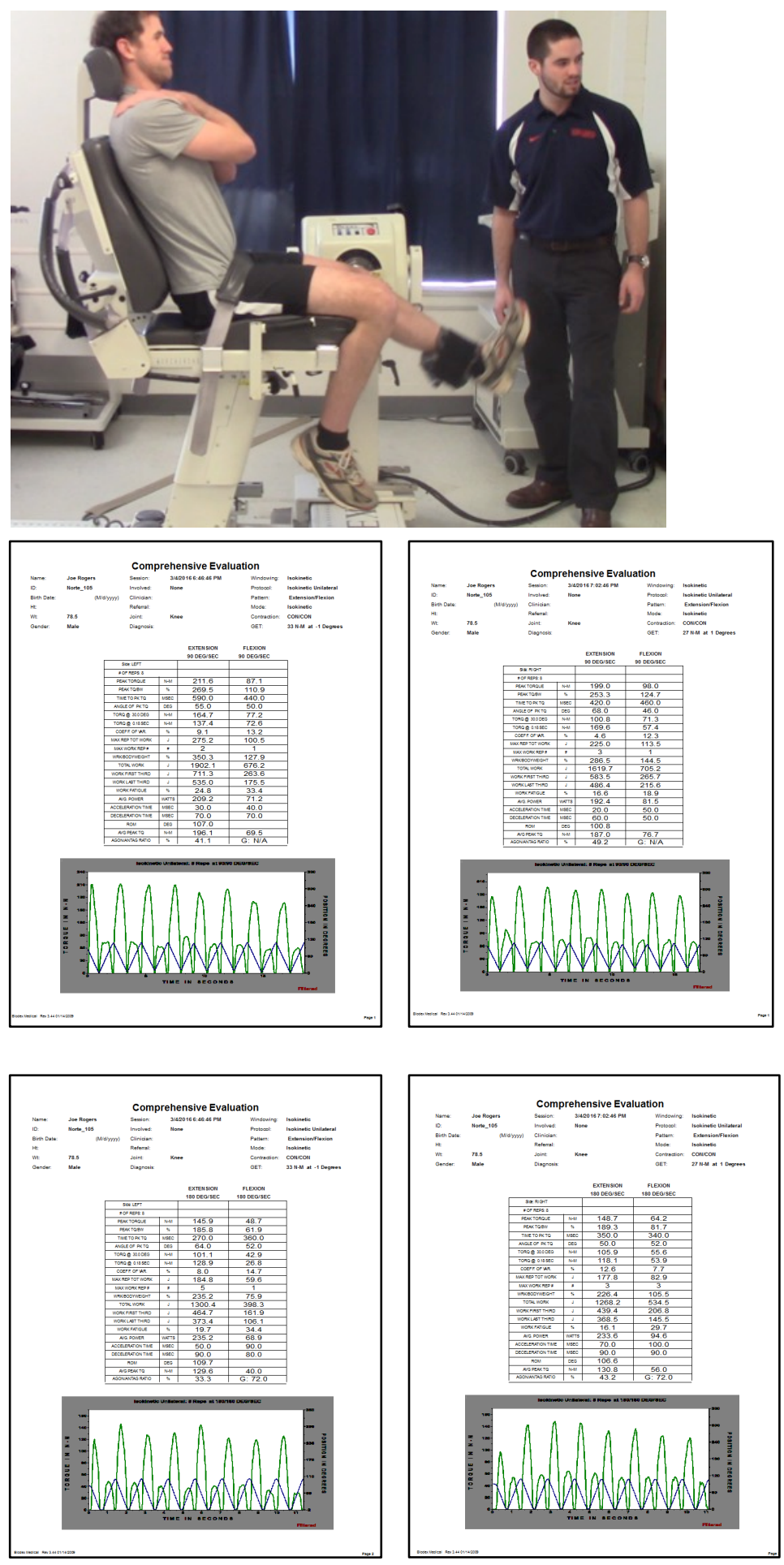

Figure C3. Patients' lower extremities were secured to a stationary dynamometry at the shank approximately $2 \mathrm{~cm}$ proximal to the lateral malleolus with knee flexed to 90 degrees. Peak torque $(\mathrm{Nm} / \mathrm{kg})$, total work $(\mathrm{j} / \mathrm{kg})$, and average power $(\mathrm{W} / \mathrm{kg})$ were recorded at 90 and 180 degrees per second from the isokinetic report (above). 


\section{Table C14. Quadriceps Superimposed Burst Technique Setup and Procedures}

1. Biodex Setup

a. Turn on the Biodex System 3

b. Allow time for calibration and attach the desired knee attachment

c. Set the away limit to 0 degrees

d. Set the toward limit to $>90$ degrees

e. Select isometric mode and press the start button

f. Confirm the Biodex arm is perpendicular to the floor using a handheld dynamometer

g. Position the back of the Biodex chair at 80 degrees

h. Connect the Biodex output wire to the MP150 (Force - channel 2)

2. AcqKnowledge Setup

a. Open AcqKnowledge 4.2.0 for Windows and select the attached MP150 unit

b. Select MP150| Acquire

i. Change the menus to "record" and "append"

ii. Change the sampling rate to $2000 \mathrm{~Hz}$

iii. Change the Acquisition Length to 30 seconds

iv. Exit the menu

c. Select MP150 | Setup Channels

i. Click the Analog tab

ii. Label Channel $2=$ Force

iii. Click the Acquire, Plot, and Values boxes

iv. Changes the sampling rate to $125 \mathrm{~Hz}$

v. Exit the menu

d. Click the start icon to confirm data acquisition and graphical representation

e. Save the data file

3. GRASS S48 Stimulator Setup

a. Turn the stimulator on

b. Confirm proper SIU8T stimulation isolator connection

i. Do not turn this unit on until ready to deliver a stimulus

c. Confirm isolation unit settings (SIU8T)

i. Constant Voltage $=$ Low

ii. Polarity $=$ Normal

iii. Stimulus Intensity $=20$

d. Confirm stimulator settings

i. $\quad$ Train Rate $=1.0$ TPS

ii. Train Duration $=10.0 \mathrm{~ms}$

iii. Stim Rate $=10.0$ PPS

iv. Delay $=1.0 \mathrm{~ms}$

v. Duration $=6.0 \mathrm{~ms}$

vi. Volts $=$ Max

vii. Output $=$ On

viii. Stim Mode $=$ Single

e. Connect electrode wires to the stimulation isolation unit (SIU8T)

4. Subject Preparation (Figure C4)

a. Place self-adhesive carbon impregnated electrodes over the proximal vastus lateralis and distal vastus medialis 
b. Position the subject in the dynamometer chair in an upright seated posture

i. Knees flexed to 90 degrees

ii. Hips flexed to 80 degrees

iii. Restrain the subject using the lap strap

iv. Engage the ankle strap $2 \mathrm{~cm}$ proximal to the lateral malleolus

c. Provide instructions on proper knee extension testing technique

i. "Sit up straight"

ii. "Do not lift your backside out of the seat"

iii. "Do not rotate or arch your back"

iv. "Do not grip the handles on the dynamometer"

v. "Cross your arms across your chest"

vi. "Concentrate on kicking out using only your quadriceps muscle - ramp up over 2 seconds, hold steady for 3 seconds, and relax"

d. Allow the subject practice trials at 25\%,50\%,75\% and $100 \%$ of their perceived maximum effort contraction

e. Confirm maximal effort and appropriate plateau in at least 2 practice trials at $100 \%$ effort before testing

5. Data Collection

a. Turn on the stimulus isolation unit (SIU8T)

b. Confirm the stimulus intensity setting on the GRASS S48 stimulator

c. Click the start button within the AcqKnowledge 4.2.0 window

d. The subject should complete a maximal knee extension contraction that matches or exceeds the torque output achieved during practice trials

e. Trigger a single stimulation pulse using the GRASS S48 stimulator when a plateau is reached at the maximal force output value

i. If no plateau is reached, abort the contraction

f. Instruct the subject to relax

g. Click the stop button within the AcqKnowledge 4.2.0 window

h. Repeat steps b-g two additional times for a total of three trials

i. Allow at least 30 seconds between trials

i. Save the data file

6. Data Processing (Figure C4)

a. Open the data file

b. Change the measurement tools to the desired options

i. Delta T

ii. Min/Max depending on limb tested

iii. Mean

c. Click Transform | Digital Filters | FIR | Low Pass

i. Frequency cutoff $=10 \mathrm{~Hz}$

ii. $\quad$ Rate of Coefficients $=$ Optimized for sampling rate and cutoff

iii. Click filter entire waveform

iv. Close the window

d. Highlight the $100 \mathrm{~ms}$ epoch immediately prior to the superimposed burst stimulus

i. Record the Mean voltage value

e. Highlight the superimposed burst epoch in the data window

i. Record the Min/Max value

f. Repeat for subsequent trials

g. Do not save before closing the data file 
Figure C4. Superimposed Burst Technique and Quadriceps Central Activation Ratio Calculation
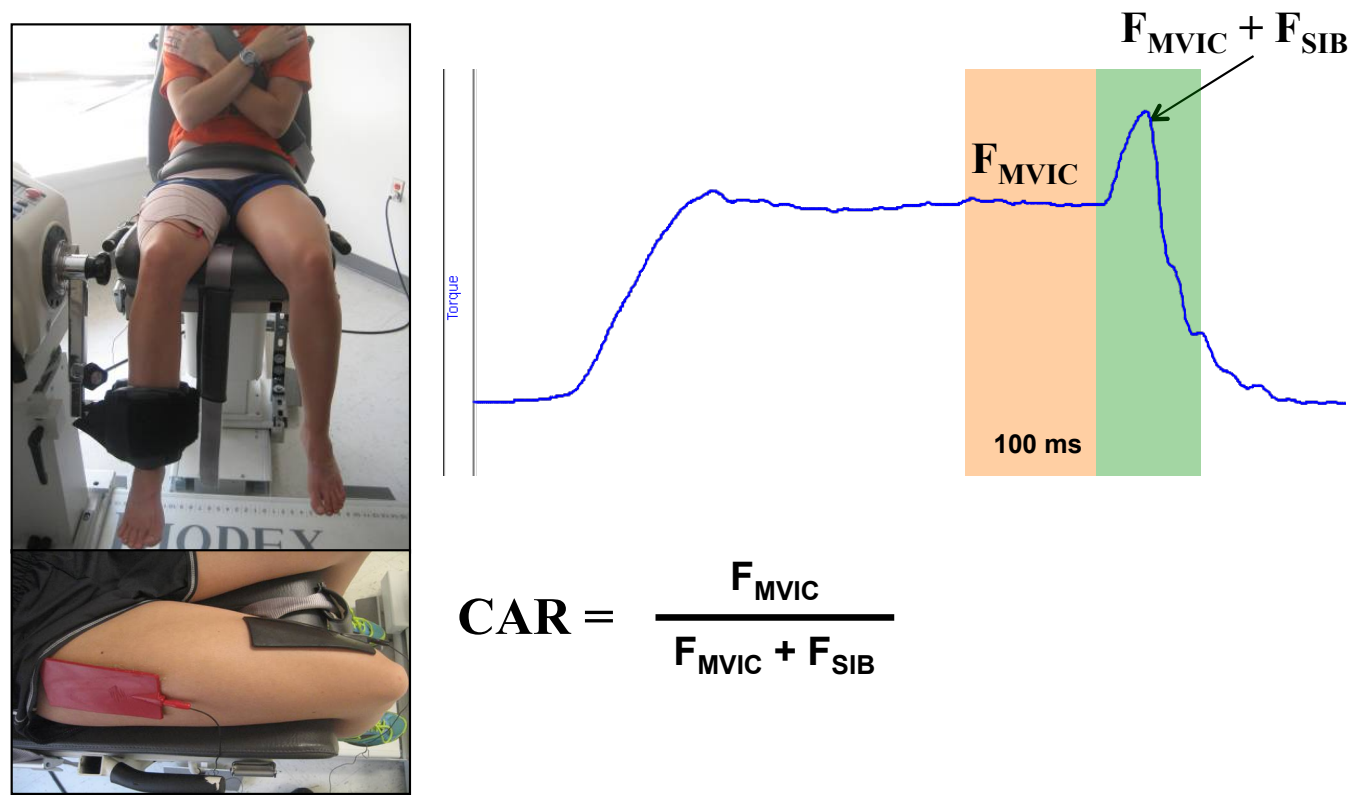

Figure C4. The superimposed burst technique was used to measure quadriceps activation.

Adhesive electrodes were applied to the anterior thigh over the proximal vastus lateralis and distal vastus medialis. Participants provided a maximal knee extension force, and an electrical stimulus was applied through the stimulating electrodes. The central activation ratio (CAR) was calculated from a $100 \mathrm{~ms}$ mean MVIC force $\left(\mathrm{F}_{\mathrm{MVIC}}\right)$ and the resulting superimposed burst force $\left(\mathrm{F}_{\mathrm{SIB}}\right)$ using the equation above. Mean MVIC was recorded from a $100 \mathrm{~ms}$ epoch approximately 1 second into each trial, and averaged across a minimum of two trials. A third MVIC trial obtained during the superimposed burst technique was used in the final averaged torque value. All force data were converted to a torque and normalized to body mass $(\mathrm{Nm} / \mathrm{kg})$. 


\section{Table C15. Quadriceps Fatigue Index Setup and Procedures}

1. Biodex Setup

a. Turn on the Biodex System 3

b. Allow time for calibration and attach the desired knee attachment

c. Set the away limit to 0 degrees

d. Set the toward limit to $>90$ degrees

e. Select isometric mode and press the start button

f. Confirm the Biodex arm is perpendicular to the floor using a handheld dynamometer

g. Position the back of the Biodex chair at 80 degrees

h. Connect the Biodex output wire to the MP150 (Force - channel 2)

2. AcqKnowledge Setup

a. Open AcqKnowledge 4.2.0 for Windows and select the attached MP150 unit

b. Select MP150| Acquire

i. Change the menus to "record" and "append"

ii. Change the sampling rate to $2000 \mathrm{~Hz}$

iii. Change the Acquisition Length to 30 seconds

iv. Exit the menu

c. Select MP150 $\rightarrow$ Setup Channels

i. Click the Analog tab

ii. Label Channel $1 \rightarrow$ Force

iii. Click the Acquire, Plot, and Values boxes

iv. Changes the sampling rate to $125 \mathrm{~Hz}$

v. Exit the menu

d. Take the following steps to set up fatigue index protocol

Label variables under the ANALOG and CALCULATION tabs. Set force channel as source for TARGET
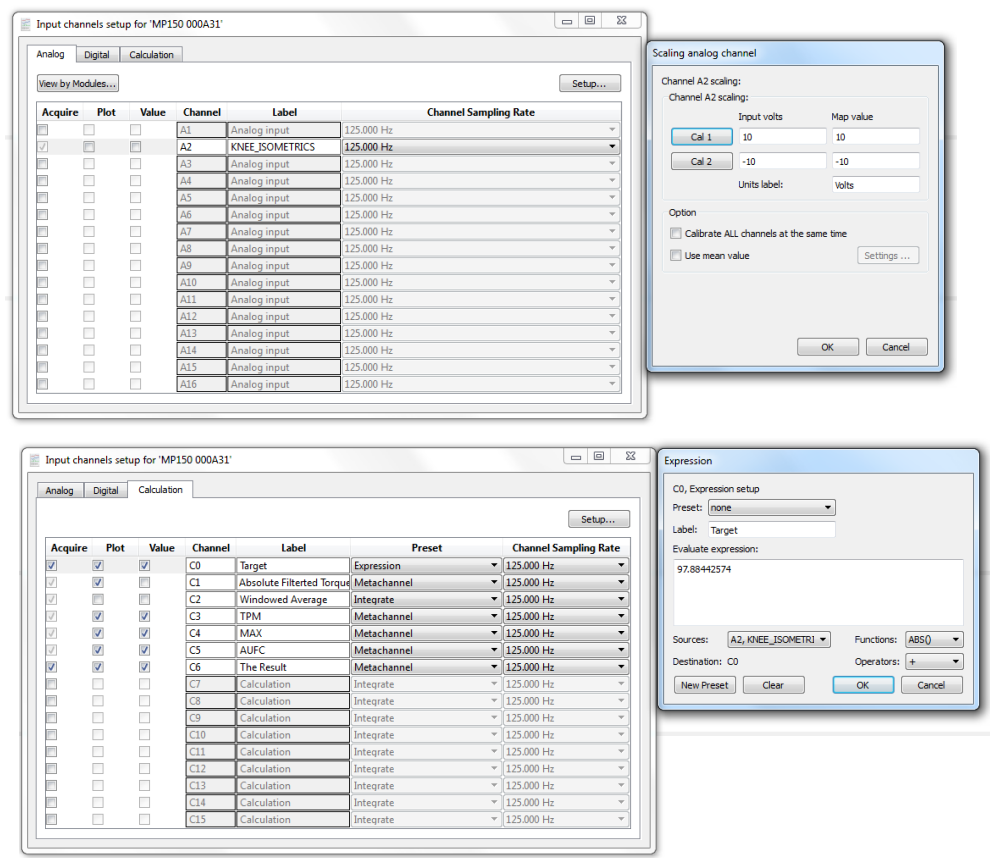
Continue down list of labels (images on left) - For each variable, select the label box|click SETUP|set up according to image on right
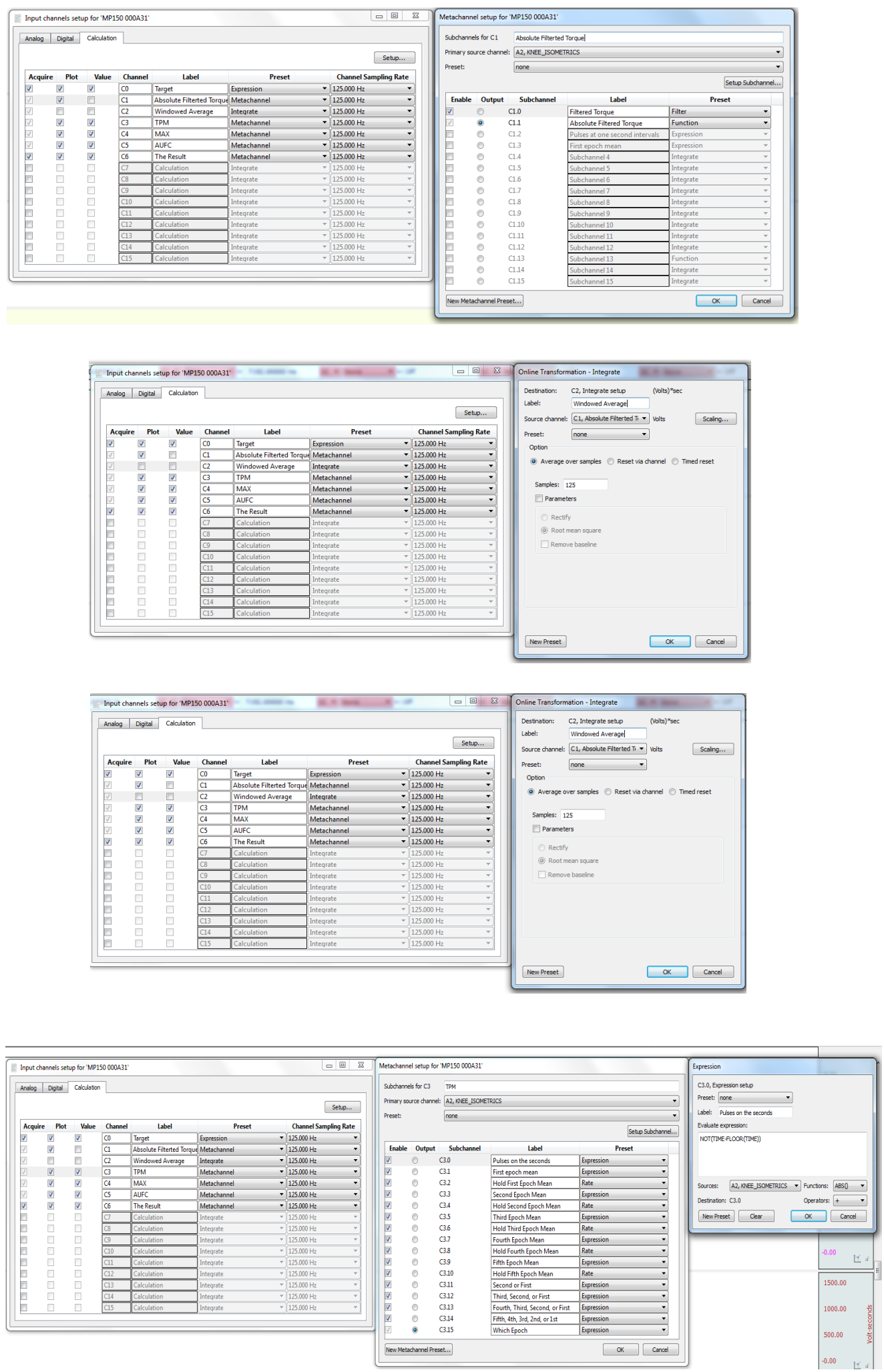

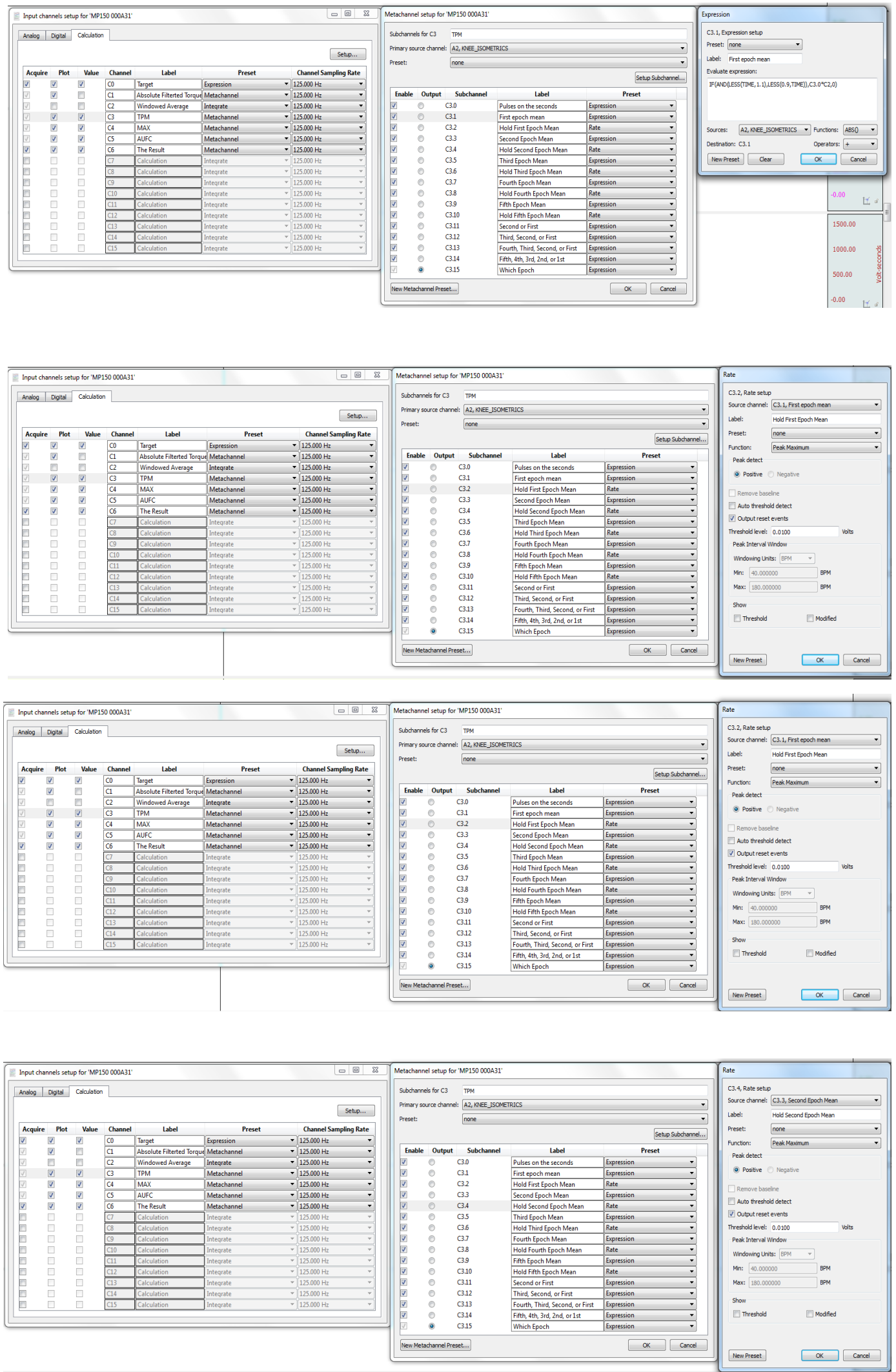

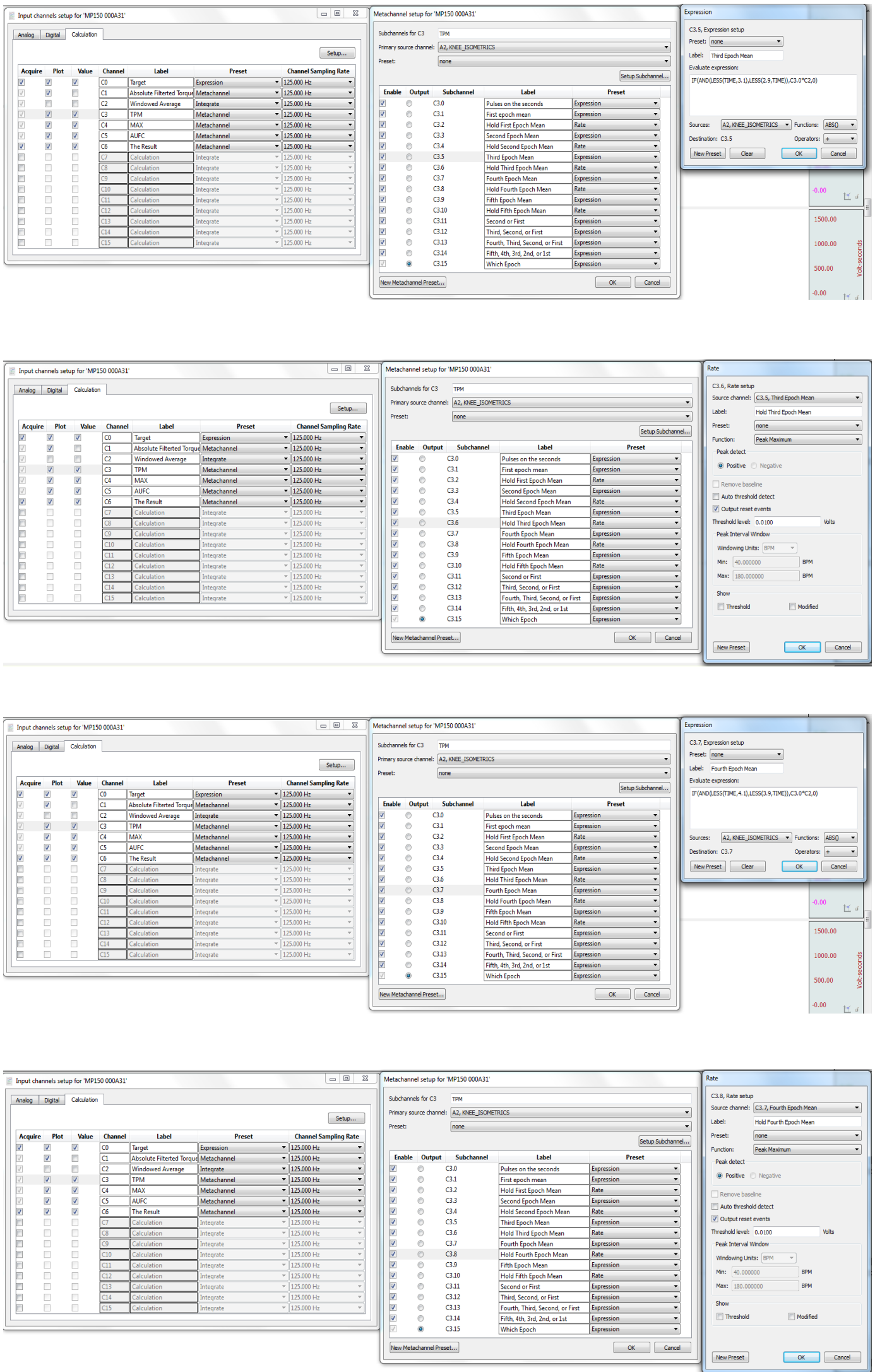

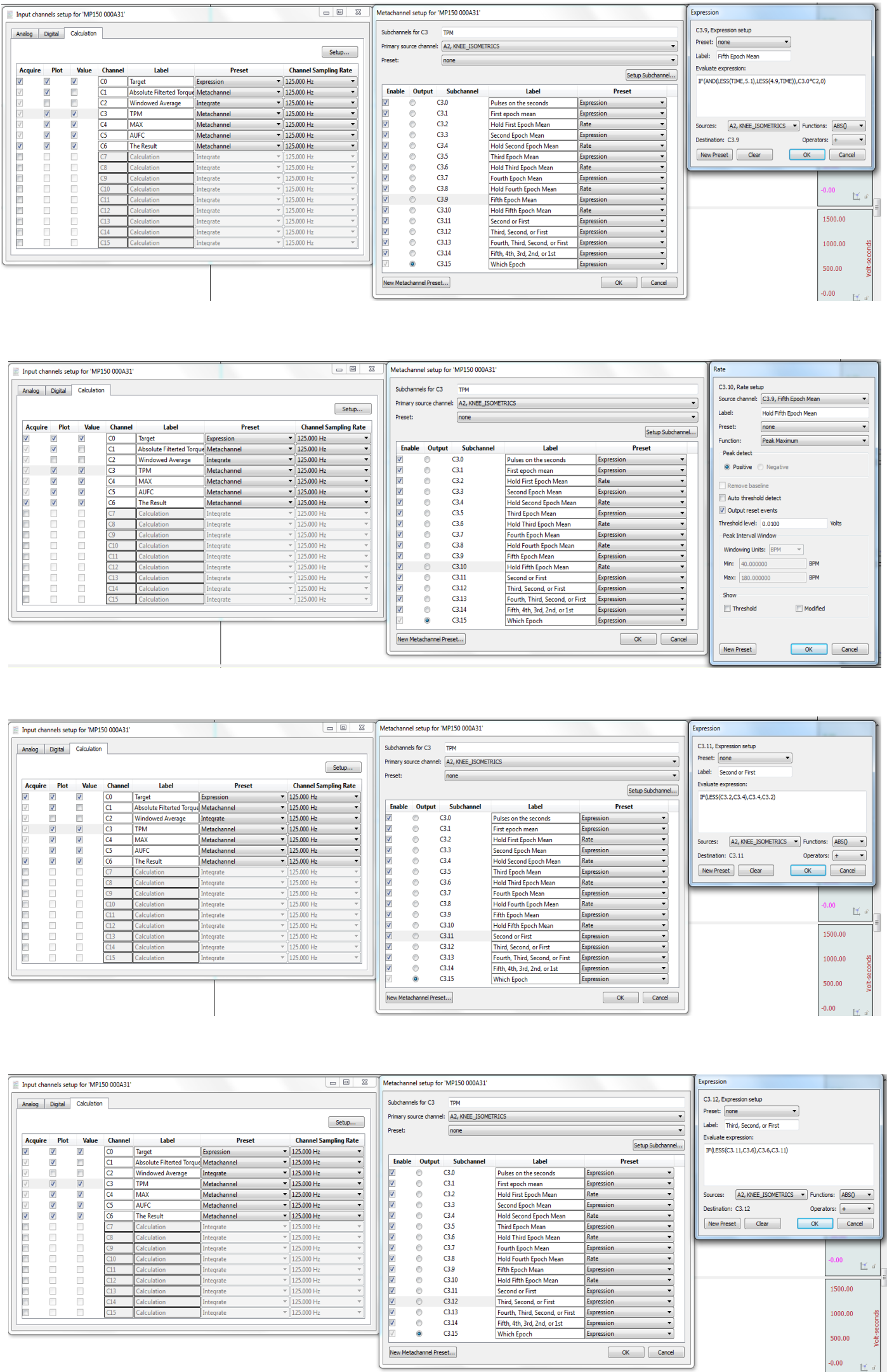

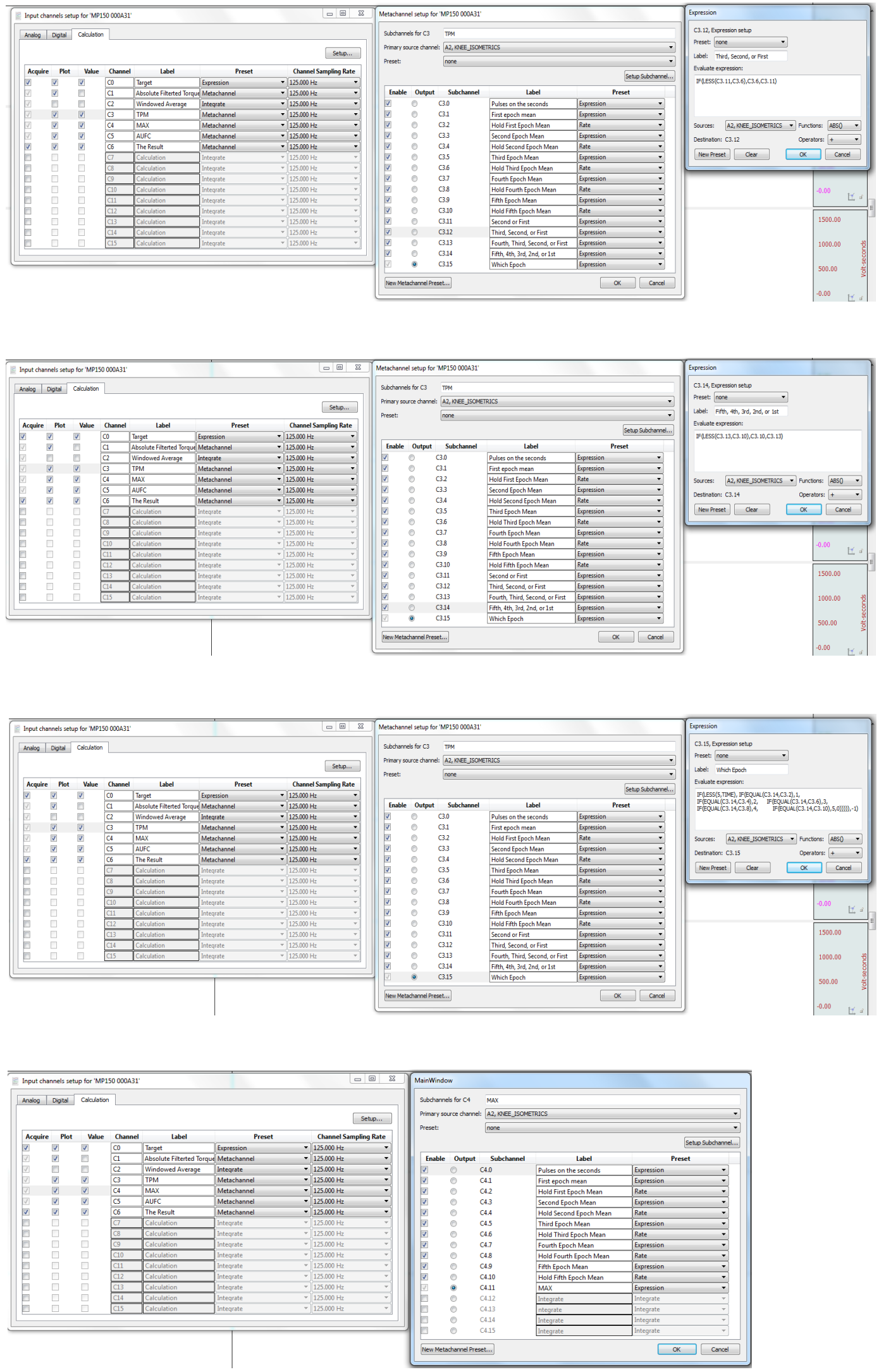

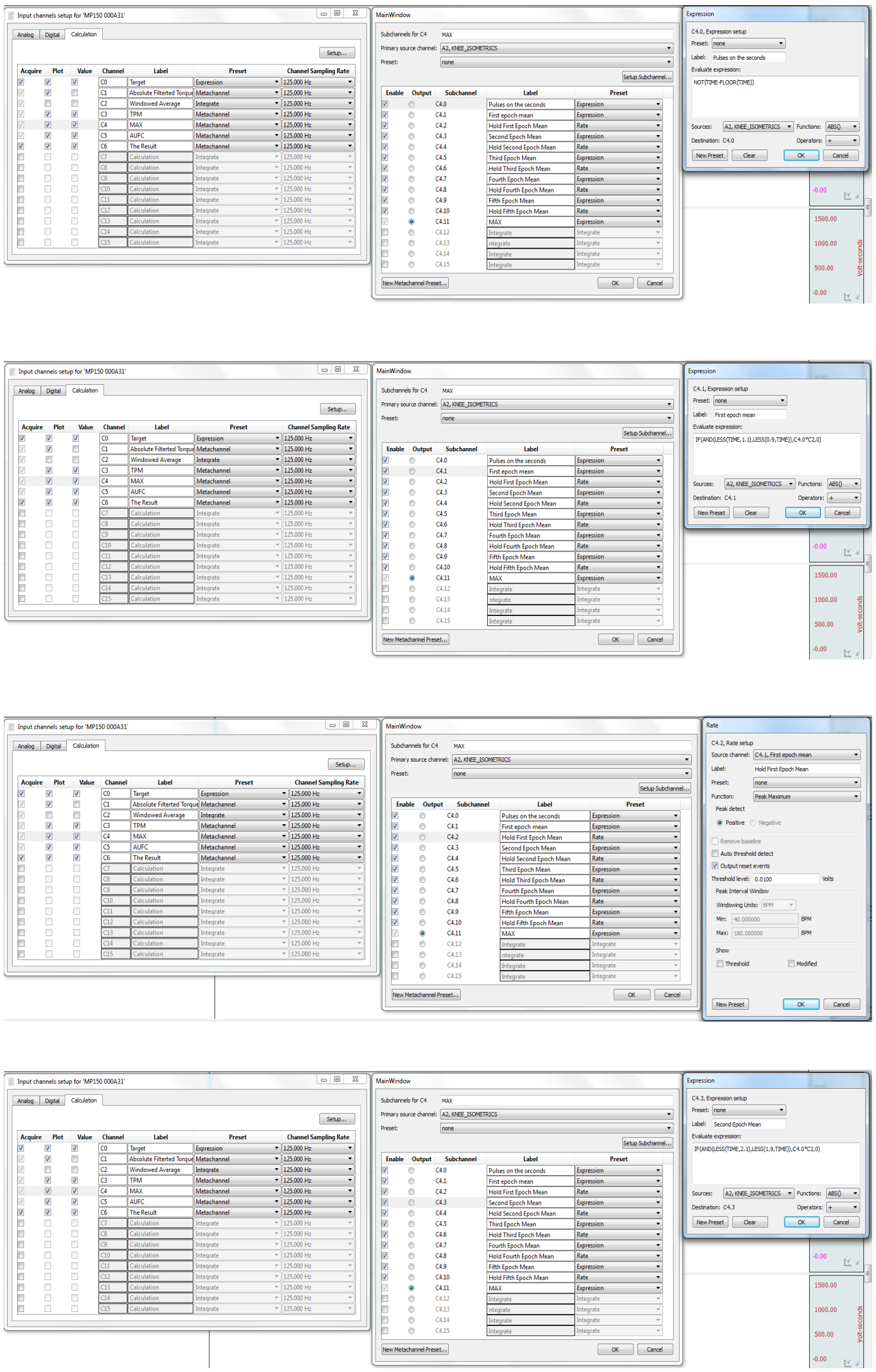

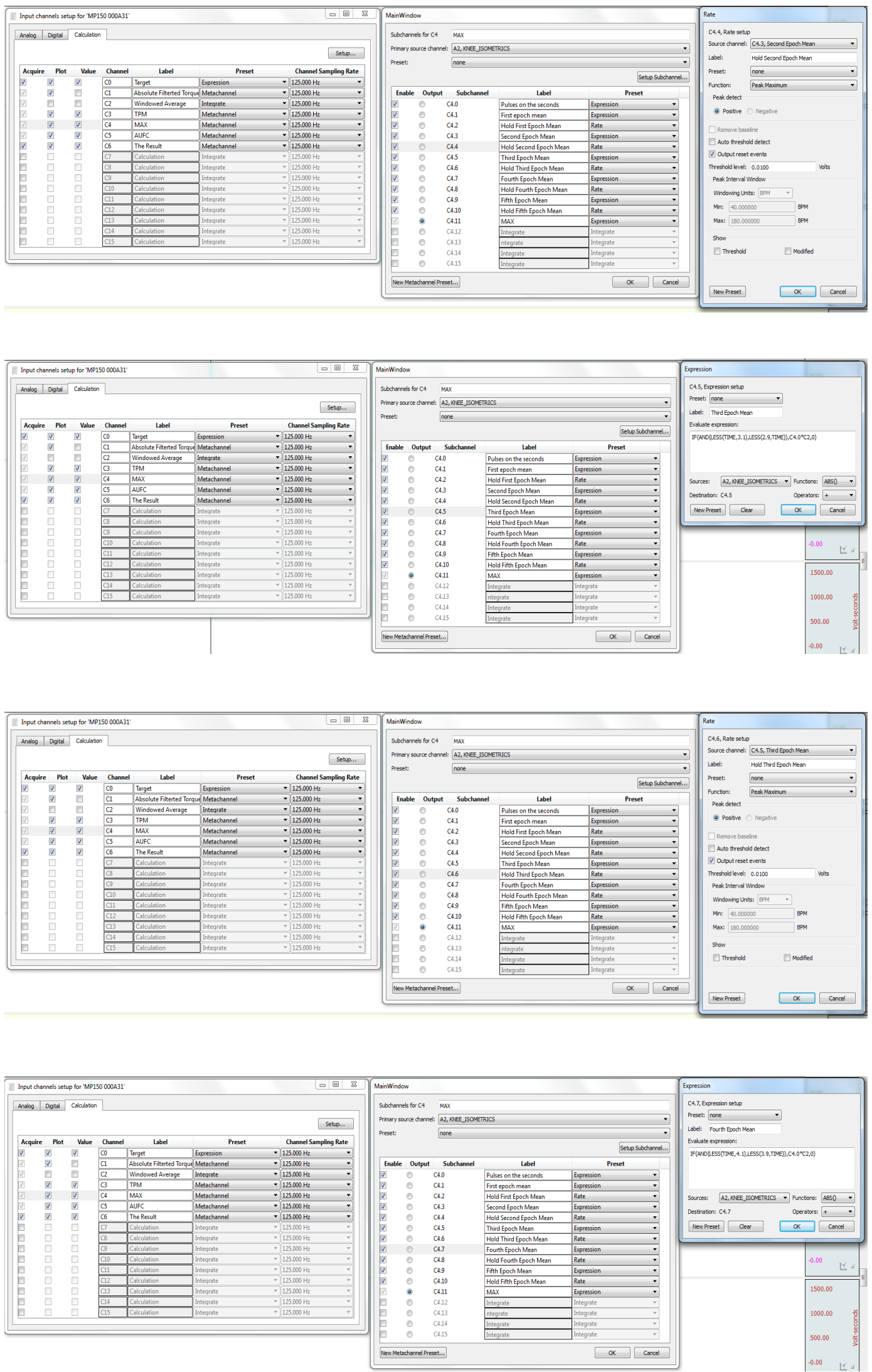

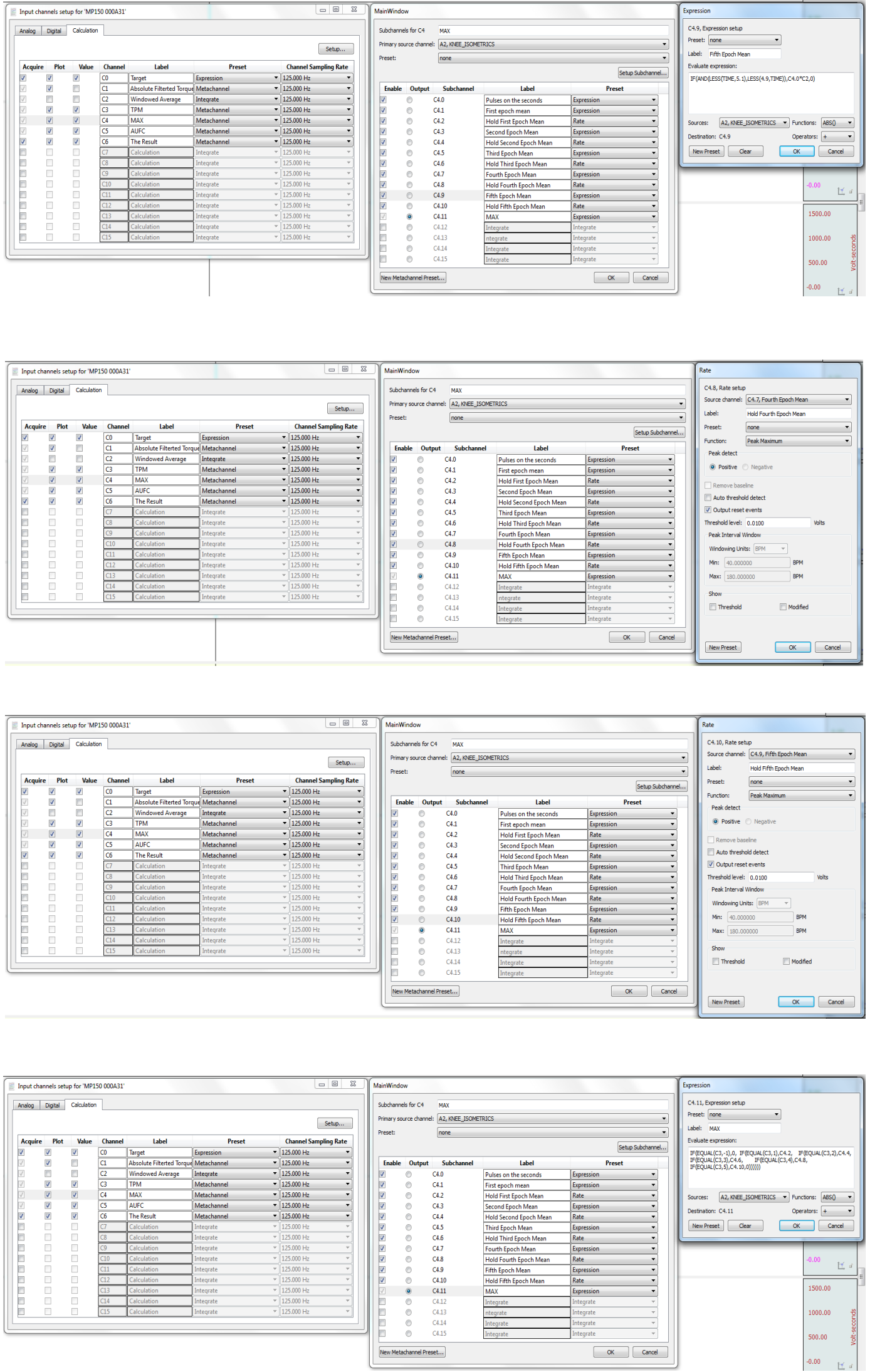

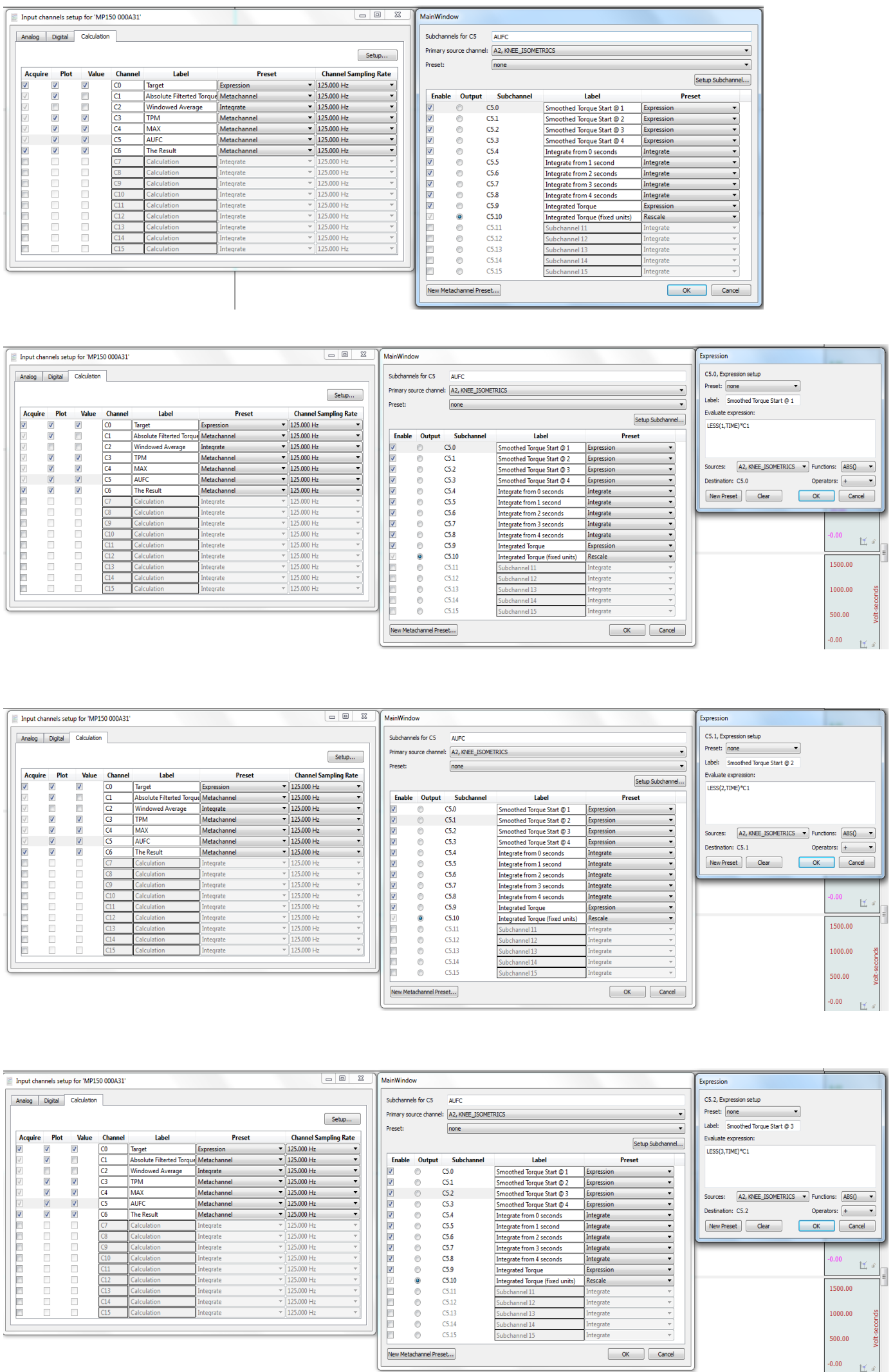

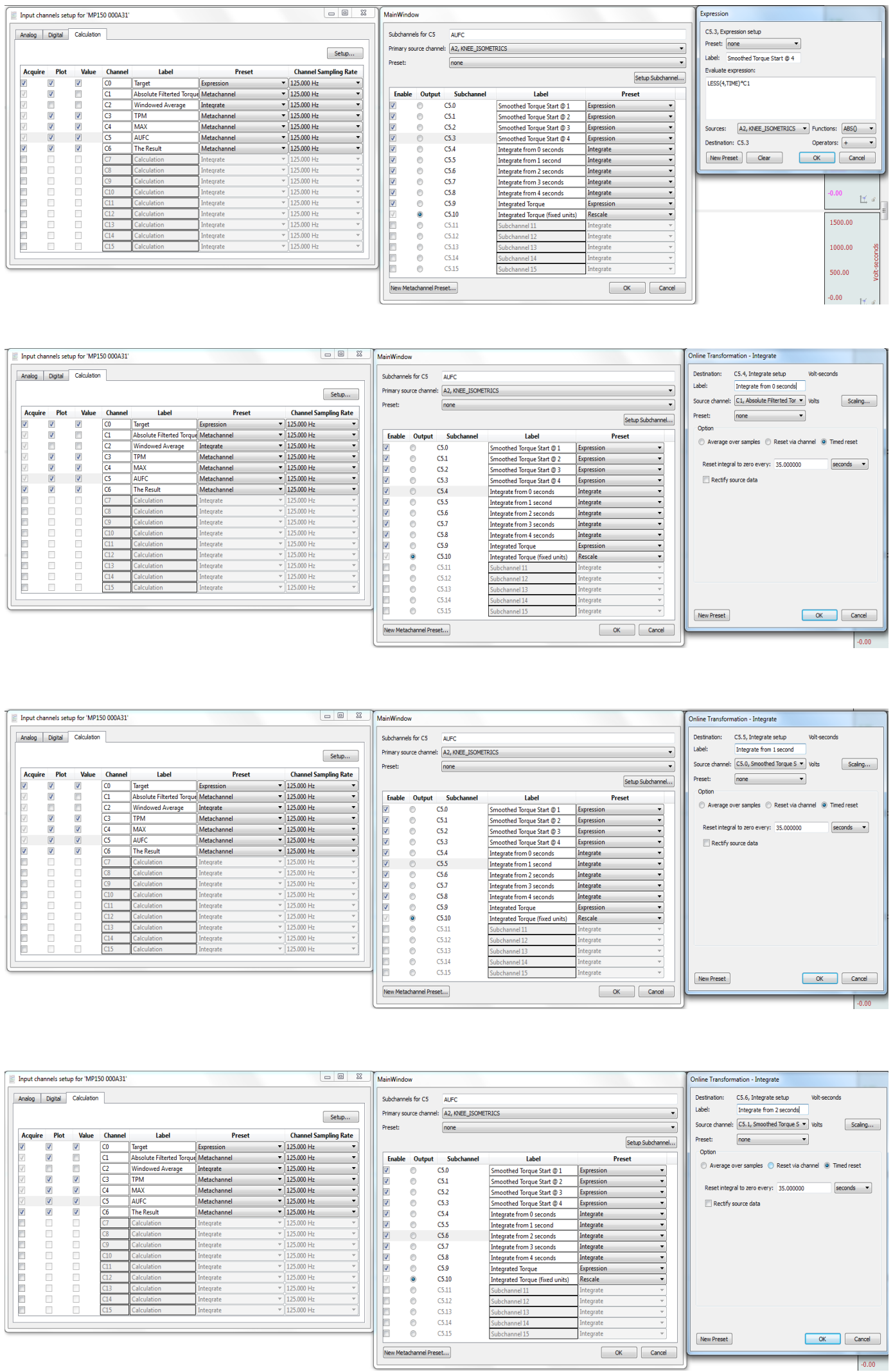

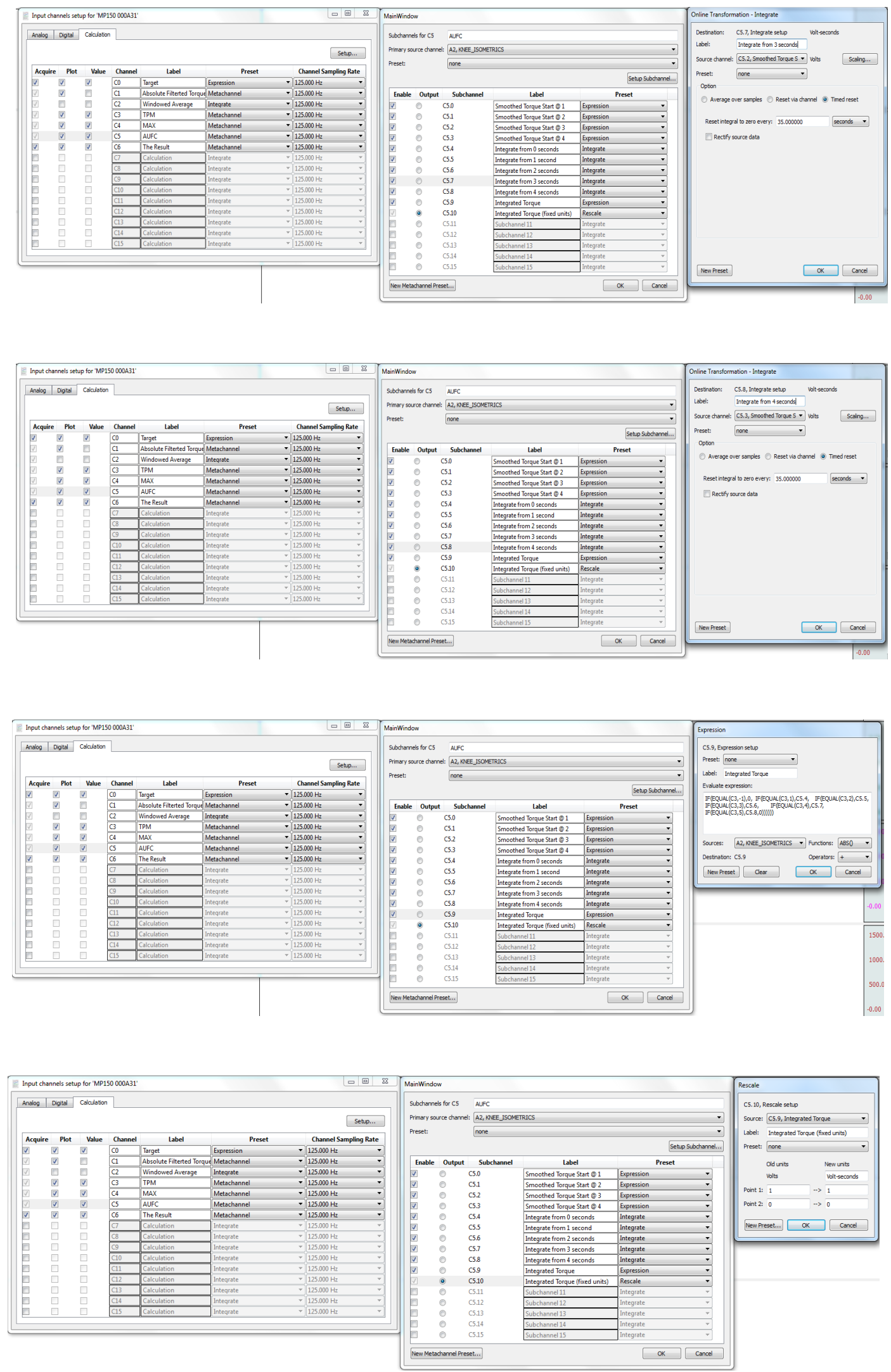

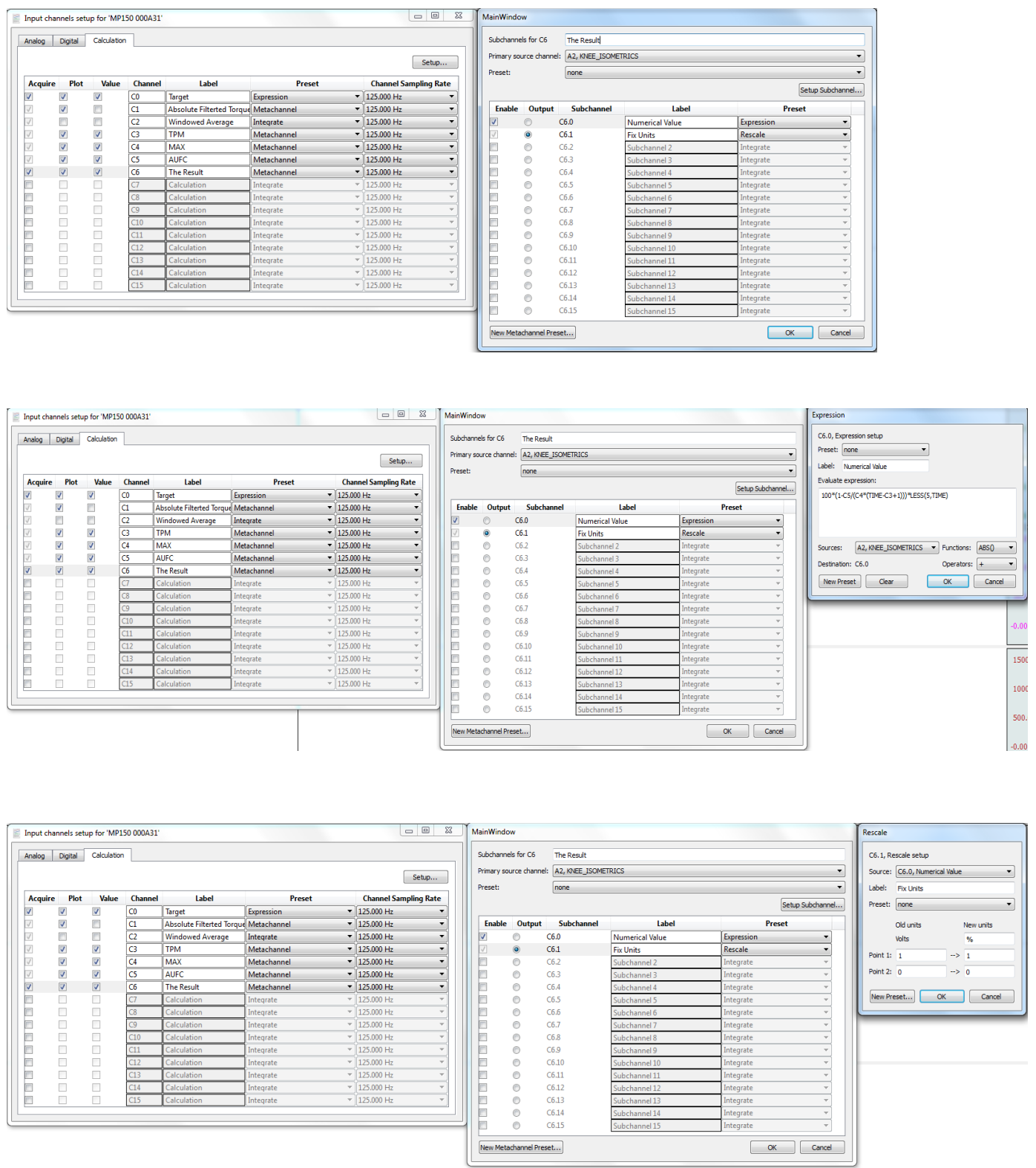

e. Click the start icon to confirm data acquisition and graphical representation

f. Save the data file

3. Data Processing (Figure C5)
a. Open file
b. Minimize force data window
c. Select "Result" from the left column
d. Highlight the right most portion of the result
e. Record value 
Figure C5. Fatigue Index Output

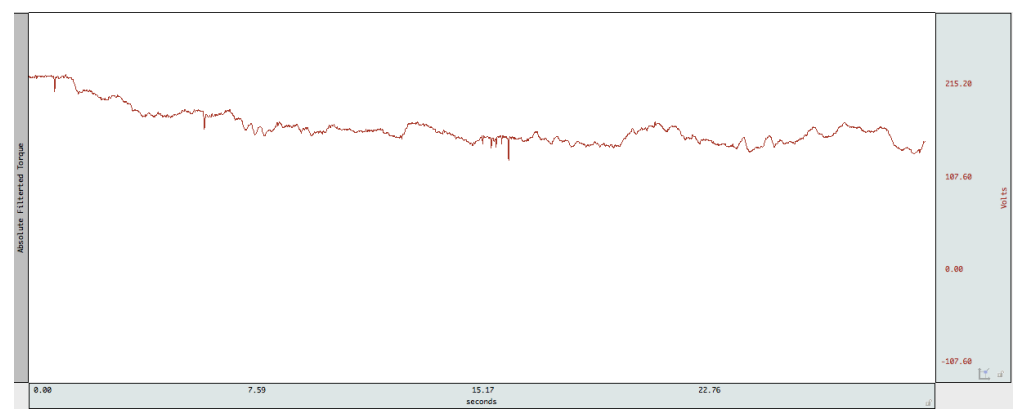

\section{Peak}

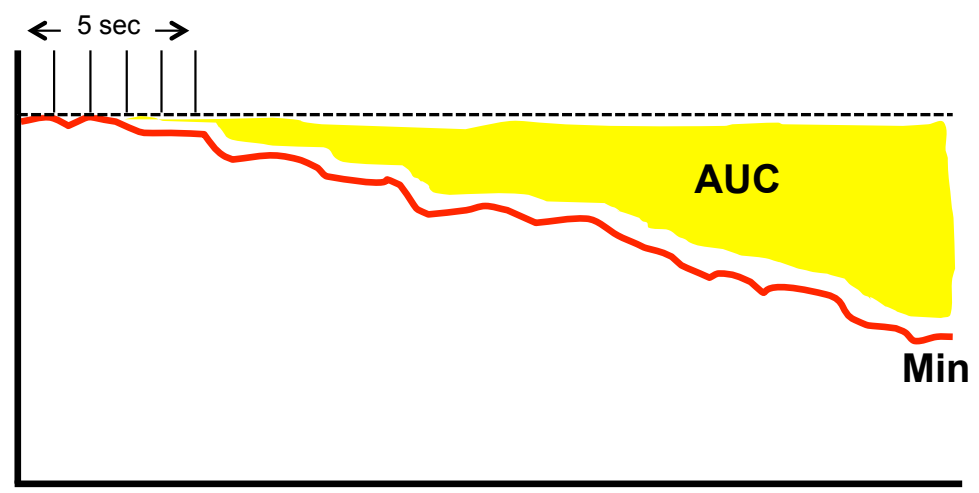

Time (30 seconds)

Figure C5. Represents a 30-second knee extension MVIC fatiguing task. Patients performed a knee extension MVIC force at 90 degrees of knee flexion, and attempted to maintain for 30 seconds. The mean torque was recorded from a series of 1 -second epochs, and the greatest torque epoch during the first 5 seconds of the trial was recorded as the maximal torque $\left(\mathrm{T}_{\mathrm{Max}}\right)$. Quadriceps FI was calculated using the area under the force-time curve (AUFC) for the entire contraction period for 0 to 30 seconds, which began at the time point of maximum muscle torque $(\mathrm{TPM})$. Fatigue index was calculated as $\mathrm{FI}=\left[1-\left(\mathrm{AUFC}_{\mathrm{TPM}-30} /\left(\mathrm{T}_{\mathrm{Max}, 0-5} \times(\mathrm{TPM}-30)\right)\right)\right] \mathrm{x} 100$. 


\section{Table C16. Transcranial Magnetic Stimulation Setup and Procedures}

1. Biopac System Setup

a. Connect an UIM100C and EMG100C to the MP150 unit

b. Connect the MP150 to the computer using a LAN wire

c. Turn on the MP150 unit and the computer

d. EMG100C settings

i. Gain $=1000$

ii. $\quad$ Filter $=$ Off

iii. $\mathrm{LP}=5 \mathrm{kHz}$

iv. $\mathrm{HP}=1.0 \mathrm{~Hz}$

2. Magstim Rapid Setup

a. Insert the footswitch connector in the "Foot Switch" port on the back of the Magstim device

b. Insert the Magstim output cable to the "Trigger Out" port on the back of the Magstim device, and to channel 3 of the UIM100C

c. Connect the output cable of the Booster Module Plus to the front of the Booster Module device and back of the Magstim device

d. Connect the stimulating coil to the Magstim device using the port on the front of the machine

e. Turn the main power switch located on the front of the Booster Module device to the $\mathrm{ON}$ position

f. Turn the Magstim device on using the ON/OFF button on the front panel

i. The Unit Power Status Indicator should remain lit throughout the testing session

g. Press the green RUN button to charge the unit and illuminate the ready indicator

3. AcqKnowledge 4.2.0 Setup (TMS Template)

a. Open AcqKnowledge 4.2.0 for Mac and select the attached MP150 unit (Laptop used for this)

b. MP150| Setup Channels $\mid$ Analog menu

i. Channel 1

1. Sample Rate $=125 \mathrm{~Hz}$

2. Label $=$ Torque

3. Check all boxes associated with this channel

ii. Channel 2

1. Sample Rate $=2000 \mathrm{~Hz}$

2. Label $=$ MEP

3. Check all boxes associated with this channel

iii. Channel 3

1. Sample Rate $=125 \mathrm{~Hz}$

2. Label $=$ TMS

3. Check all boxes associated with this channel

c. MP150| Set Up Acquisition

i. Change menus to "Record" and "Append"

ii. Sample Rate $=2000 \mathrm{~Hz}$

iii. Acquisition Length $=80 \mathrm{msec}$

d. Open the data journal and graph window

e. Click the start button to confirm proper setup 
4. AcqKnowledge 4.2.0 Setup (Torque_TMS Template)

a. Open AcqKnowledge 4.2.0 for Windows and select the attached MP150 unit

b. MP150| Setup Channels $\mid$ Analog menu

i. Channel 2

1. Sample Rate $=200 \mathrm{~Hz}$

2. Label $=$ Force

3. Check all boxes associated with this channel

c. MP150| Setup Channels $\mid$ Calculation menu

i. Channel 0

1. Label $=\mathrm{C} 0-$ Expression

2. Preset $=$ Expression

3. Sample Rate $=200 \mathrm{~Hz}$

ii. Setup (C0 - Expression)

1. Preset $=$ None

2. Label $=\mathrm{C} 0-$ Expression

3. Evaluate Expression $=<<$ Insert voltage $=5 \%$ MVIC $>>$

4. Sources $=$ A2, Force

5. Functions $=\mathrm{ABS}()$

6. Operators $=+$

iii. Setup $(\mathrm{C} 1-$ Math $)$

1. Preset $=$ None

2. Label $=\mathrm{C} 1-$ Math

3. Source $1=$ A2, Force

4. Operation $=\mathrm{a}$

5. Source $2=\mathrm{K}$, Constant

6. Constant $=0.05$

d. MP150| Set Up Acquisition

i. Change menus to "Record" and "Append"

ii. Sample Rate $=200 \mathrm{~Hz}$

iii. Acquisition Length $=10 \mathrm{~min}$

e. Open the data journal and graph window

f. Click the start button to confirm proper setup

5. Subject Preparation (Figure C6)

a. Identify the vastus medialis during isometric knee extension

i. Shave the area

ii. Debride with an abrasive pad

iii. Clean with isopropyl alcohol

b. Place 2 EMG electrodes in the prepared area

i. Parallel to the muscle fiber orientation

ii. Interelectrode distance of $2 \mathrm{~cm}$

c. Identify an area on the distal anteromedial tibia for the ground electrode

i. Shave the area

ii. Debride with an abrasive pad

iii. Clean with isopropyl alcohol

d. Place 1 EMG electrode in the prepared area

e. Position the subject in the dynamometer chair in an upright seated posture

i. Knees flexed to 90 degrees

ii. Hips flexed to 80 degrees

iii. Restrain the subject using the lap strap

iv. Engage the ankle strap $2 \mathrm{~cm}$ proximal to the lateral malleolus 
f. Attach the leads from the EMG100C unit to the active and reference electrodes

i. $\quad$ Proximal active $=$ Red lead

ii. $\quad$ Distal active $=$ White lead

iii. Reference $=$ Black lead

g. Place a pre-marked nylon swim-cap on the subject's head

i. Marked with two perpendicular lines from:

1. Left tragi to right tragi

2. External occipital protuberance to midline near the midline

ii. Marked with dots in a grid pattern $-1 \mathrm{~cm}$ apart

h. Provide earplugs for the subject to be worn throughout testing

i. Ask the subject to relax, breathe normally, fold hands in lap, and keep head back against the headrest.

i. Subjects were asked to "kick" to a red line, indicating 5\% MVIC, then to relax the leg after the stimulus was delivered

j. Turn off the lights and any additional unnecessary electronics

6. Data Collection Procedures (Figure C6)

a. Position the stimulating coil over the contralateral homunculus of the testing limb near the central sulcus

i. Tape piece of paper under central curve of stimulating coil with vertical line drawn extending to the superior and inferior border

ii. Move coil in $0.5-1 \mathrm{~cm}$ increments, aligning the drawn line with a dot on the swim cap

iii. The homunculus should correspond the area near the cross of the perpendicular lines on the swim-cap

b. Set the Magstim output to $60 \%$

c. Click the START button within the Acqknowledge software (on each computer)

d. Depress the Magstim footswitch

e. Press and hold the trigger on the stimulating coil

f. Review the data for motor response in the EMG (MEP) channel

i. If positive for motor response

1. Record MEP amplitude in journal, noting the corresponding location from the vertex (i.e. $-6,-2$ )

2. Repeat stimulus in radius around this point

3. Continue until the maximum MEP amplitude has been found, and a $1-\mathrm{cm}$ radius around this point has been assessed

4. Decrease the stimulation intensity by $5 \%$, and re-stimulate

5. When no response is observed, increase the stimulation intensity by $1 \%$ and repeat stimulus until MEP is detected

6. Wait at least 10 seconds between stimulations

ii. If negative for motor response, re-position and repeat at same stimulus intensity

1. Continue until $1-\mathrm{cm}$ radius has been stimulated

2. If no response, increase stimulus intensity by $5 \%$, and restimulate

g. Continue to decrease the stimulus intensity until MEP is measured

i. Once confirmed, deliver 10 stimulations

ii. If positive for MEP in at least $50 \%$ of trials, end testing

iii. If negative for MEP in at least $60 \%$ of trials, increase stimulus intensity $1 \%$ and test again. 
7. Data Processing

a. During testing, record the peak-to-peak amplitude (P-P), time from stimulus artifact to onset of MEP (delta T), and time for each MEP in the journal of AcqKnowledge - coordinates should be documented when searching for the ideal coil position, or "hotspot," during subsequent testing

b. Record the active motor threshold (AMT) as the intensity required for $50 \%$ success during 10 consecutive trials

c. Record P-P, Delta T, and time for MEPs detected at $120 \%, 130 \%$, and $140 \%$ MEP

i. Record the stimulus intensity at each percentage

ii. Record a minimum of five acceptable trials 
Figure C6. Transcranial Magnetic Stimulation Procedures
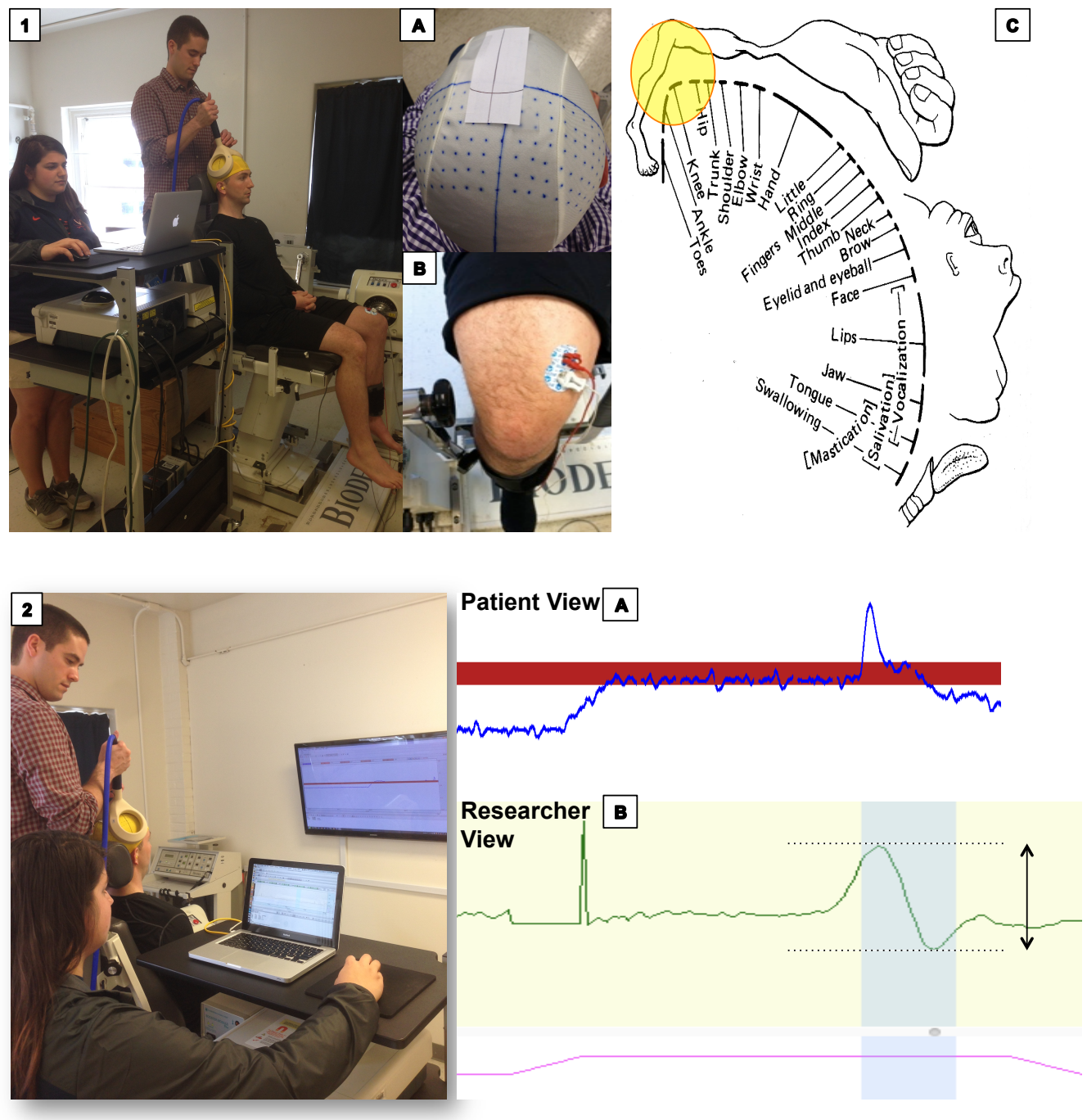

Figure C6. Image 1 illustrates TMS setup, including (A) swim cap to use a grid for optimal coil placement, (B) surface EMG electrode placement over the vastus medialis, and (C) location of stimulus over the motor cortex as depicted by the motor homunculus. Image 2 illustrates the (A) $5 \%$ MVIC force matching task performed by the patient, and (B) recording of peak-peak motor evoked potential. Stimulus intensity was reduced until a MEP was no longer detectable. The lowest intensity able to elicit a measurable MEP was recorded as the active motor threshold. 
Table C17. Sample Size Estimate

\begin{tabular}{lcccc}
\hline Outcome & $\begin{array}{c}\text { Minimal } \\
\text { Detectable } \\
\text { Change (MDC) }\end{array}$ & $\begin{array}{c}\text { Expected } \\
\text { Variance (SD) }\end{array}$ & $\begin{array}{c}\text { Sample } \\
\text { Estimate }\end{array}$ & Source \\
\hline MVIC (N) & 47.8 & \pm 59.1 & 24 & $\begin{array}{l}\text { Park and } \\
\text { Hopkins, 2013 }\end{array}$ \\
Fatigue (\%) & 11.0 & \pm 9.0 & 11 & Poulsen, 2015 \\
CAR (\%) & 6.0 & \pm 6.0 & 16 & $\begin{array}{l}\text { Park and } \\
\text { Hopkins, 2014 }\end{array}$ \\
H:M ratio & 0.30 & \pm 0.22 & 11 & $\begin{array}{l}\text { Hopkins and } \\
\text { Waggie, 2003 }\end{array}$ \\
AMT (\%) & 8.4 & \pm 8.0 & 14 & Luc, 2013 \\
\hline
\end{tabular}




\section{APPENDIX D}

\section{Additional Results}

\section{MANUSCRIPT I}

Table D1. ANOVA comparison of group and limb for normalized knee extension MVIC torque

\begin{tabular}{lrrrrr} 
Dependent Variable: MVIC & \multicolumn{7}{c}{} \\
\hline Source & $\begin{array}{c}\text { Type III Sum } \\
\text { of Squares }\end{array}$ & df & Mean Square & \multicolumn{1}{c}{ F } & \multicolumn{1}{c}{ Sig. } \\
\hline Corrected Model & $24.092^{\mathrm{a}}$ & 7 & 3.442 & 8.353 & .000 \\
Intercept & 732.679 & 1 & 732.679 & 1778.177 & .000 \\
Group_Small_Stack & 14.376 & 3 & 4.792 & 11.630 & .000 \\
Limb & 2.269 & 1 & 2.269 & 5.508 & .020 \\
Group_Small_Stack * & 5.962 & 3 & 1.987 & 4.823 & .003 \\
Limb & & & & & \\
Error & 79.936 & 194 & .412 & & \\
Total & 1254.001 & 202 & & & \\
Corrected Total & 104.028 & 201 & & & \\
\hline
\end{tabular}

a. $\mathrm{R}$ Squared $=.232$ (Adjusted $\mathrm{R}$ Squared $=.204$ )

Table D2. Post hoc analyses from ANOVA comparison of group and limb for normalized knee extension MVIC torque

\begin{tabular}{|c|c|c|c|c|c|c|c|}
\hline & \multirow[b]{2}{*}{ (D) Group Small Stack } & \multirow[b]{2}{*}{ (J) Group Small Stack } & \multirow{2}{*}{$\begin{array}{c}\text { Mean } \\
\text { Difference (I- } \\
\mathrm{J})\end{array}$} & \multirow[b]{2}{*}{ Std. Error } & \multirow[b]{2}{*}{ Sig. } & \multicolumn{2}{|c|}{ 95\% Confidence Interval } \\
\hline & & & & & & Lower Bound & Upper Bound \\
\hline \multirow[t]{12}{*}{$\overline{L S D}$} & Healthy & $6-12$ month & .5032 & .11421 & .000 & .2780 & .7285 \\
\hline & & $2-10$ year & $.5094^{\circ}$ & .11769 & .000 & .2773 & .7415 \\
\hline & & $O A$ & $.8998^{*}$ & .18562 & .000 & .5337 & 1.2659 \\
\hline & $6-12$ month & Healthy & $-.5032^{\circ}$ & .11421 & .000 & -.7285 & -.2780 \\
\hline & & $2-10$ year & .0062 & .11370 & .957 & -.2181 & .2304 \\
\hline & & $O A$ & $.3966^{*}$ & .18311 & .032 & .0354 & .7577 \\
\hline & $2-10$ year & Healthy & $-.5094^{\circ}$ & .11769 & .000 & -.7415 & -.2773 \\
\hline & & 6-12 month & -.0062 & .11370 & .957 & -.2304 & .2181 \\
\hline & & OA & $.3904^{*}$ & .18530 & .036 & .0249 & .7559 \\
\hline & $\overline{O A}$ & Healthy & -.8998 & .18562 & .000 & -1.2659 & -.5337 \\
\hline & & 6-12 month & $-.3966^{\circ}$ & .18311 & .032 & -.7577 & -.0354 \\
\hline & & $2-10$ year & $-.3904^{*}$ & .18530 & .036 & -.7559 & -.0249 \\
\hline \multirow[t]{3}{*}{ Dunnett $t(2 \text {-sided })^{b}$} & 6-12 month & Healthy & $-.5032^{\circ}$ & .11421 & .000 & -.7753 & -.2311 \\
\hline & $2-10$ year & Healthy & $-.5094^{\circ}$ & .11769 & .000 & -.7898 & -.2290 \\
\hline & OA & Healthy & $-.8998^{\circ}$ & .18562 & .000 & -1.3420 & -.4576 \\
\hline
\end{tabular}


Table D3. Between limb comparisons of normalized knee extension MVIC torque per group

\begin{tabular}{|c|c|c|c|c|c|c|c|c|c|c|}
\hline \multirow{3}{*}{\multicolumn{2}{|c|}{ Group_Small_Stack }} & & \multicolumn{5}{|c|}{ Paired Differences } & \multirow{4}{*}{$\frac{\mathrm{t}}{1.379}$} & \multirow[b]{3}{*}{$\mathrm{df}$} & \multirow{3}{*}{$\begin{array}{l}\text { Sig. (2- } \\
\text { tailed) }\end{array}$} \\
\hline & & & \multirow{2}{*}{$\frac{\text { Mean }}{.08702}$} & \multirow{3}{*}{$\begin{array}{c}\begin{array}{c}\text { Std. } \\
\text { Deviation }\end{array} \\
.34571\end{array}$} & \multirow{2}{*}{$\begin{array}{c}\begin{array}{c}\text { Std. Error } \\
\text { Mean }\end{array} \\
.06312\end{array}$} & \multicolumn{2}{|c|}{$\begin{array}{l}95 \% \text { Confidence Interval of } \\
\text { the Difference }\end{array}$} & & & \\
\hline & & & & & & Lower & Upper & & & \\
\hline Healthy & Pair 1 & $\begin{array}{l}\text { Norm_MVIC_Torque_Inv } \\
\overline{\text { Norm_MVIC_Torque_Un }}\end{array}$ & .08702 & & & -.04206 & .21611 & & 29 & .179 \\
\hline $6-12$ month & Pair 1 & $\begin{array}{l}\text { Norm_MVIC_Torque_Inv } \\
\text { Norm_MVIC_Torque_Un }\end{array}$ & -.71558 & .55245 & .09474 & -.90834 & -.52282 & -7.553 & 33 & .000 \\
\hline 2-10 year & Pair 1 & $\begin{array}{l}\text { Norm_MVIC_Torque_Inv } \\
\overline{\text { Norm_MVIC_Torque_Un }}\end{array}$ & -.12192 & .29003 & .05295 & -.23022 & -.01362 & -2.303 & 29 & .029 \\
\hline OA & Pair 1 & $\begin{array}{l}\text { Norm_MVIC_Torque_Inv } \\
\bar{N} \text { Norm_MVIC_Torque_Un }\end{array}$ & -.18086 & .48965 & .17312 & -.59022 & .22849 & -1.045 & 7 & .331 \\
\hline
\end{tabular}

Table D4. ANOVA comparison of group and limb for quadriceps fatigue index

\begin{tabular}{|c|c|c|c|c|c|}
\hline \multicolumn{6}{|c|}{ Dependent Variable: Fatigue } \\
\hline Source & $\begin{array}{c}\text { Type III Sum } \\
\text { of Squares }\end{array}$ & df & Mean Square & $\mathrm{F}$ & Sig. \\
\hline Corrected Model & $1484.459^{\mathrm{a}}$ & 7 & 212.066 & 3.090 & .004 \\
\hline Intercept & 56713.668 & 1 & 56713.668 & 826.366 & .000 \\
\hline Group_Small_Stack & 1001.253 & 3 & 333.751 & 4.863 & .003 \\
\hline Limb & 17.025 & 1 & 17.025 & .248 & .619 \\
\hline $\begin{array}{l}\text { Group_Small_Stack * } \\
\text { Limb }\end{array}$ & 372.213 & 3 & 124.071 & 1.808 & .147 \\
\hline Error & 13245.635 & 193 & 68.630 & & \\
\hline Total & 95313.823 & 201 & & & \\
\hline Corrected Total & 14730.094 & 200 & & & \\
\hline
\end{tabular}

a. R Squared $=.101$ (Adjusted $R$ Squared $=.068$ )

Table D5. Post hoc analyses from ANOVA comparison of group and limb for quadriceps fatigue index

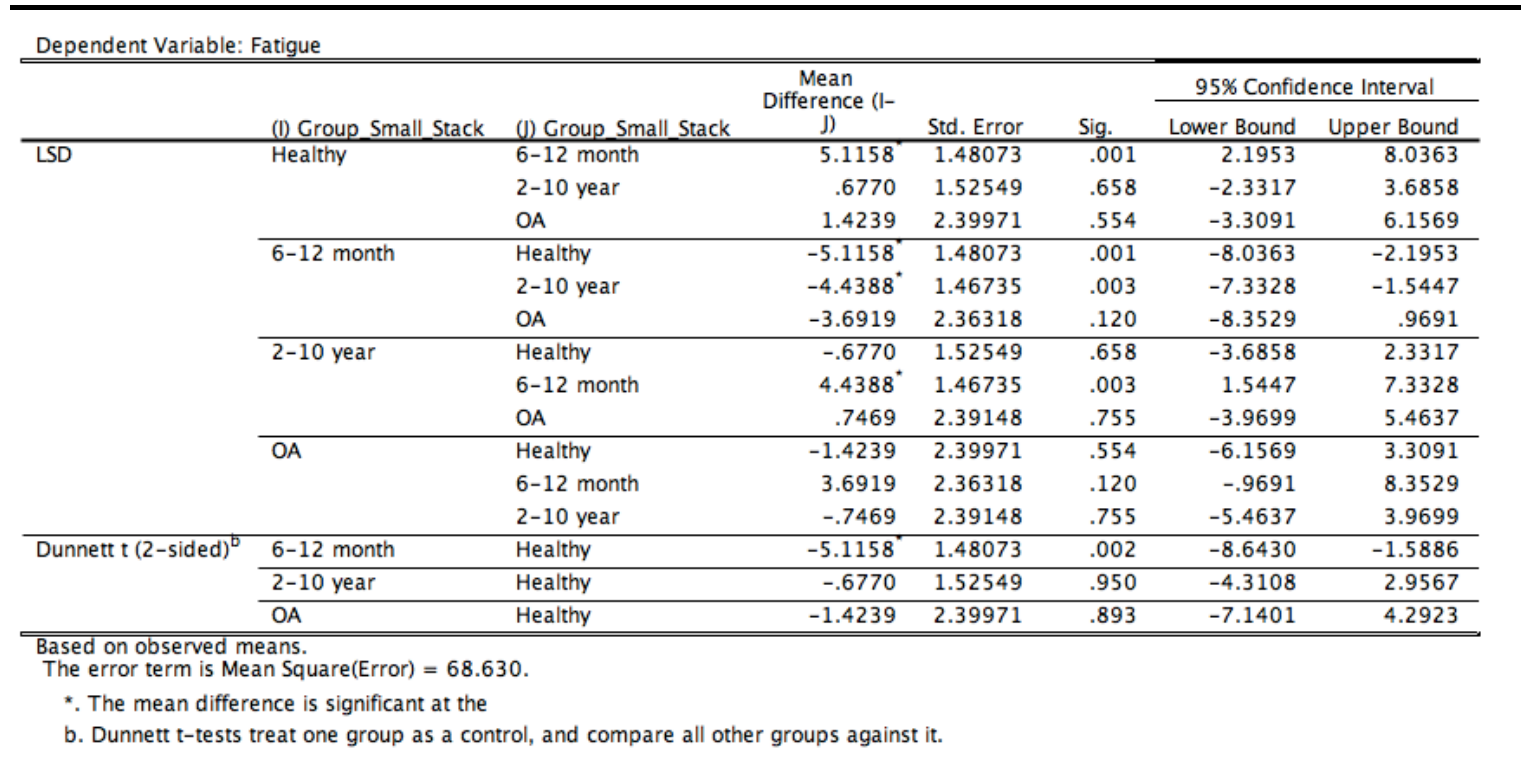


Table D6. Between limb comparisons of quadriceps fatigue index per group

\begin{tabular}{|c|c|c|c|c|c|c|c|c|c|c|}
\hline \multirow{3}{*}{\multicolumn{2}{|c|}{ Group Small Stack }} & & \multicolumn{5}{|c|}{ Paired Differences } & \multirow[b]{3}{*}{$\mathrm{t}$} & \multirow[b]{3}{*}{ df } & \multirow{3}{*}{$\begin{array}{l}\text { Sig. (2- } \\
\text { tailed) }\end{array}$} \\
\hline & & & \multirow[b]{2}{*}{ Mean } & \multirow{2}{*}{$\begin{array}{c}\text { Std. } \\
\text { Deviation }\end{array}$} & \multirow{2}{*}{$\begin{array}{l}\text { Std. Error } \\
\text { Mean }\end{array}$} & \multicolumn{2}{|c|}{$\begin{array}{c}95 \% \text { Confidence Interval of } \\
\text { the Difference }\end{array}$} & & & \\
\hline & & & & & & Lower & Upper & & & \\
\hline Healthy & Pair 1 & Fatigue_Inv - Fatigue_Un & -.41663 & 6.22494 & 1.15594 & -2.78447 & 1.95121 & -.360 & 28 & .721 \\
\hline $6-12$ month & Pair 1 & Fatigue_Inv - Fatigue_Un & -5.20853 & 8.90125 & 1.52655 & -8.31432 & -2.10274 & -3.412 & 33 & .002 \\
\hline $2-10$ year & Pair 1 & Fatigue_Inv - Fatigue_Un & .65618 & 6.04112 & 1.10295 & -1.59961 & 2.91197 & .595 & 29 & .557 \\
\hline OA & Pair 1 & Fatigue_Inv - Fatigue_Un & 1.84308 & 4.64721 & 1.64304 & -2.04208 & 5.72825 & 1.122 & 7 & .299 \\
\hline
\end{tabular}

Table D7. ANOVA comparison of group and limb for quadriceps central activation ratio

\begin{tabular}{lrrrrr}
\hline Dependent Variable: CAR & \multicolumn{7}{c}{} \\
\hline Source & $\begin{array}{c}\text { Type III Sum } \\
\text { of Squares }\end{array}$ & df & Mean Square & \multicolumn{1}{c}{ F } & \multicolumn{1}{c}{ Sig. } \\
\hline Corrected Model & $.181^{\mathrm{a}}$ & 7 & .026 & 3.149 & .004 \\
Intercept & 114.572 & 1 & 114.572 & 13922.128 & .000 \\
Group_Small_Stack & .168 & 3 & .056 & 6.817 & .000 \\
Limb & .001 & 1 & .001 & .137 & .711 \\
Group_Small_Stack* & .009 & 3 & .003 & .361 & .781 \\
Limb & 1.588 & 193 & .008 & & \\
Error & 166.211 & 201 & & & \\
Total & 1.770 & 200 & & & \\
Corrected Total & & & & & \\
\hline
\end{tabular}

a. $\mathrm{R}$ Squared $=.103$ (Adjusted R Squared $=.070$ )

Table D8. Post hoc analyses from ANOVA comparison of group and limb for quadriceps central activation ratio

\begin{tabular}{|c|c|c|c|c|c|c|c|}
\hline \multicolumn{8}{|c|}{ Dependent Variable: CAR } \\
\hline & \multirow[b]{2}{*}{ (I) Group Small Stack } & \multirow[b]{2}{*}{ (J) Group Small Stack } & \multirow{2}{*}{$\begin{array}{c}\text { Mean } \\
\text { Difference (I- } \\
\mathrm{J})\end{array}$} & \multirow[b]{2}{*}{ Std. Error } & \multirow[b]{2}{*}{ Sig. } & \multicolumn{2}{|c|}{ 95\% Confidence Interval } \\
\hline & & & & & & Lower Bound & Upper Bound \\
\hline \multirow[t]{12}{*}{ LSD } & Healthy & $6-12$ month & .0715 & .01621 & .000 & .0396 & .1035 \\
\hline & & 2-10 year & .0320 & .01670 & .057 & -.0009 & .0650 \\
\hline & & OA & .0143 & .02628 & .588 & -.0376 & .0661 \\
\hline & 6-12 month & Healthy & $-.0715^{\circ}$ & .01621 & .000 & -.1035 & -.0396 \\
\hline & & 2-10 year & $-.0395^{*}$ & .01607 & .015 & -.0712 & -.0078 \\
\hline & & OA & $-.0573^{*}$ & .02588 & .028 & -.1083 & -.0062 \\
\hline & $2-10$ year & Healthy & -.0320 & .01670 & .057 & -.0650 & .0009 \\
\hline & & 6-12 month & $.0395^{\circ}$ & .01607 & .015 & .0078 & .0712 \\
\hline & & $\mathrm{OA}$ & -.0177 & .02619 & .499 & -.0694 & .0339 \\
\hline & $\mathrm{OA}$ & Healthy & -.0143 & .02628 & .588 & -.0661 & .0376 \\
\hline & & 6-12 month & $.0573^{*}$ & .02588 & .028 & .0062 & .1083 \\
\hline & & 2-10 year & .0177 & .02619 & .499 & -.0339 & .0694 \\
\hline \multirow[t]{3}{*}{ Dunnett $t(2-\text { sided })^{b}$} & $6-12$ month & Healthy & $-.0715^{\circ}$ & .01621 & .000 & -.1102 & -.0329 \\
\hline & $2-10$ year & Healthy & -.0320 & .01670 & .147 & -.0718 & .0078 \\
\hline & $\mathrm{OA}$ & Healthy & -.0143 & .02628 & .915 & -.0769 & .0483 \\
\hline
\end{tabular}


Table D9. Between limb comparisons of quadriceps fatigue index per group

\begin{tabular}{|c|c|c|c|c|c|c|c|c|c|c|}
\hline \multirow{3}{*}{\multicolumn{2}{|c|}{ Group Small Stack }} & & \multicolumn{5}{|c|}{ Paired Differences } & \multirow[b]{3}{*}{ t } & \multirow[b]{3}{*}{ df } & \multirow{3}{*}{$\begin{array}{l}\text { Sig. (2- } \\
\text { tailed) }\end{array}$} \\
\hline & & & \multirow[b]{2}{*}{ Mean } & \multirow{2}{*}{$\begin{array}{c}\text { Std. } \\
\text { Deviation }\end{array}$} & \multirow{2}{*}{$\begin{array}{l}\text { Std. Error } \\
\text { Mean }\end{array}$} & \multicolumn{2}{|c|}{$\begin{array}{c}95 \% \text { Confidence Interval of } \\
\text { the Difference }\end{array}$} & & & \\
\hline & & & & & & Lower & Upper & & & \\
\hline Healthy & Pair 1 & CAR_Inv - CAR_Un & .01331 & .05220 & .00969 & -.00654 & .03317 & 1.373 & 28 & .181 \\
\hline $6-12$ month & Pair 1 & CAR_Inv - CAR_Un & -.02628 & .07879 & .01351 & -.05377 & .00122 & -1.945 & 33 & .060 \\
\hline $2-10$ year & Pair 1 & CAR_Inv - CAR_Un & -.00478 & .06627 & .01210 & -.02952 & .01997 & -.395 & 29 & .696 \\
\hline$O A$ & Pair 1 & CAR_Inv - CAR_Un & -.00273 & .06042 & .02136 & -.05324 & .04778 & -.128 & 7 & .902 \\
\hline
\end{tabular}

Table D10. ANOVA comparison of group and limb for normalized quadriceps Hoffmann reflex

\begin{tabular}{lrrrrr}
\hline Dependent Variable: HM & \multicolumn{7}{c}{} \\
\hline Source & $\begin{array}{c}\text { Type III Sum } \\
\text { of Squares }\end{array}$ & \multicolumn{1}{c}{ df } & Mean Square & \multicolumn{1}{c}{ F } & \multicolumn{1}{c}{ Sig. } \\
\hline Corrected Model & $.251^{\mathrm{a}}$ & 7 & .036 & 1.221 & .293 \\
Intercept & 4.656 & 1 & 4.656 & 158.790 & .000 \\
Group_Small_Stack & .216 & 3 & .072 & 2.454 & .065 \\
Limb & .000 & 1 & .000 & .011 & .916 \\
Group_Small_Stack * & .022 & 3 & .007 & .253 & .859 \\
Limb & & & & & \\
Error & 5.161 & 176 & .029 & & \\
Total & 11.487 & 184 & & & \\
Corrected Total & 5.411 & 183 & & & \\
\hline
\end{tabular}

a. R Squared $=.046$ (Adjusted R Squared $=.008$ )

Table D11. Post hoc analyses from ANOVA comparison of group and limb for normalized quadriceps Hoffmann reflex

\begin{tabular}{|c|c|c|c|c|c|c|c|}
\hline & \multirow[b]{2}{*}{ (1) Group_Small_Stack } & \multirow[b]{2}{*}{ (1) Group_Small Stack } & \multirow{2}{*}{$\begin{array}{c}\text { Mean } \\
\text { Difference (I- } \\
\mathrm{J})\end{array}$} & \multirow[b]{2}{*}{ Std. Error } & \multirow[b]{2}{*}{ Sig. } & \multicolumn{2}{|c|}{ 95\% Confidence Interval } \\
\hline & & & & & & Lower Bound & Upper Bound \\
\hline \multirow[t]{12}{*}{ LSD } & Healthy & $6-12$ month & -.0340 & .03259 & .298 & -.0983 & .0303 \\
\hline & & $2-10$ year & -.0605 & .03328 & .071 & -.1261 & .0052 \\
\hline & & $\mathrm{OA}$ & $-.1372^{*}$ & .05527 & .014 & -.2462 & -.0281 \\
\hline & 6-12 month & Healthy & .0340 & .03259 & .298 & -.0303 & .0983 \\
\hline & & $2-10$ year & -.0264 & .03079 & .392 & -.0872 & .0343 \\
\hline & & OA & -.1031 & .05380 & .057 & -.2093 & .0030 \\
\hline & $2-10$ year & Healthy & .0605 & .03328 & .071 & -.0052 & .1261 \\
\hline & & 6-12 month & .0264 & .03079 & .392 & -.0343 & .0872 \\
\hline & & $\mathrm{OA}$ & -.0767 & .05423 & .159 & -.1837 & .0303 \\
\hline & OA & Healthy & $.1372^{\circ}$ & .05527 & .014 & .0281 & .2462 \\
\hline & & 6-12 month & .1031 & .05380 & .057 & -.0030 & .2093 \\
\hline & & $2-10$ year & .0767 & .05423 & .159 & -.0303 & .1837 \\
\hline \multirow[t]{3}{*}{ Dunnett $\mathrm{t}(2-\text { sided })^{\mathrm{b}}$} & 6-12 month & Healthy & .0340 & .03259 & .609 & -.0435 & .1116 \\
\hline & $2-10$ year & Healthy & .0605 & .03328 & .179 & -.0188 & .1397 \\
\hline & $\mathrm{OA}$ & Healthy & $.1372^{\circ}$ & .05527 & .039 & .0056 & .2687 \\
\hline
\end{tabular}


Table D12. Between limb comparisons of normalized quadriceps Hoffmann reflex per group

\begin{tabular}{|c|c|c|c|c|c|c|c|c|c|c|}
\hline \multirow{3}{*}{\multicolumn{2}{|c|}{ Group Small Stack }} & & \multicolumn{5}{|c|}{ Paired Differences } & \multirow[b]{3}{*}{$\mathrm{t}$} & \multirow[b]{3}{*}{ df } & \multirow{3}{*}{$\begin{array}{l}\text { Sig. (2- } \\
\text { tailed) }\end{array}$} \\
\hline & & & \multirow[b]{2}{*}{ Mean } & \multirow{2}{*}{$\begin{array}{c}\text { Std. } \\
\text { Deviation }\end{array}$} & \multirow{2}{*}{$\begin{array}{l}\text { Std. Error } \\
\text { Mean }\end{array}$} & \multicolumn{2}{|c|}{$\begin{array}{l}95 \% \text { Confidence Interval of } \\
\text { the Difference }\end{array}$} & & & \\
\hline & & & & & & Lower & Upper & & & \\
\hline Healthy & Pair 1 & HM_Inv - HM_Un & -.00028 & .08727 & .01820 & -.03802 & .03746 & -.015 & 22 & .988 \\
\hline $6-12$ month & Pair 1 & HM_Inv - HM_Un & .02957 & .11048 & .01984 & -.01096 & .07009 & 1.490 & 30 & .147 \\
\hline $2-10$ year & Pair 1 & HM_Inv - HM_Un & .02434 & .09167 & .01702 & -.01053 & .05921 & 1.430 & 28 & .164 \\
\hline OA & Pair 1 & HM_Inv - HM_Un & -.03864 & .20631 & .08423 & -.25515 & .17788 & -.459 & 5 & .666 \\
\hline
\end{tabular}

Table D13. ANOVA comparison of group and limb for quadriceps active motor threshold

\begin{tabular}{lrrrrr}
\hline Dependent Variable: AMT & \multicolumn{7}{c}{} \\
\hline Source & $\begin{array}{c}\text { Type III Sum } \\
\text { of Squares }\end{array}$ & \multicolumn{1}{c}{ df } & Mean Square & \multicolumn{1}{c}{ F } & \multicolumn{1}{c}{ Sig. } \\
\hline Corrected Model & $1725.564^{\mathrm{a}}$ & 7 & 246.509 & 4.399 & .000 \\
Intercept & 251555.698 & 1 & 251555.698 & 4489.207 & .000 \\
Group_Small_Stack & 1632.479 & 3 & 544.160 & 9.711 & .000 \\
Limb & 55.999 & 1 & 55.999 & .999 & .319 \\
Group_Small_Stack * & 37.476 & 3 & 12.492 & .223 & .880 \\
Limb & & & & & \\
Error & 9694.171 & 173 & 56.036 & & \\
Total & 350316.000 & 181 & & & \\
Corrected Total & 11419.735 & 180 & & & \\
\hline
\end{tabular}

a. R Squared $=.151$ (Adjusted R Squared $=.117$ )

Table D14. Post hoc analyses from ANOVA comparison of group and limb for quadriceps active motor threshold

\begin{tabular}{|c|c|c|c|c|c|c|c|}
\hline \multicolumn{8}{|c|}{ Dependent Variable: AMT } \\
\hline & \multirow[b]{2}{*}{ (1) Group_Small_Stack } & \multirow[b]{2}{*}{ (J) Group Small Stack } & \multirow{2}{*}{$\begin{array}{c}\text { Mean } \\
\text { Difference (I- } \\
\text { J) }\end{array}$} & \multirow[b]{2}{*}{ Std. Error } & \multirow[b]{2}{*}{ Sig. } & \multicolumn{2}{|c|}{ 95\% Confidence Interval } \\
\hline & & & & & & Lower Bound & Upper Bound \\
\hline \multirow[t]{12}{*}{ LSD } & Healthy & $6-12$ month & -6.5778 & 1.47148 & .000 & -9.4822 & -3.6735 \\
\hline & & 2-10 year & $-3.7025^{*}$ & 1.50642 & .015 & -6.6758 & -.7292 \\
\hline & & $O A$ & $-10.1163^{*}$ & 2.24474 & .000 & -14.5469 & -5.6857 \\
\hline & 6-12 month & Healthy & $6.5778^{\circ}$ & 1.47148 & .000 & 3.6735 & 9.4822 \\
\hline & & $2-10$ year & $2.8753^{\circ}$ & 1.35212 & .035 & .2066 & 5.5441 \\
\hline & & OA & -3.5385 & 2.14425 & .101 & -7.7707 & .6938 \\
\hline & $2-10$ year & Healthy & $3.7025^{\circ}$ & 1.50642 & .015 & .7292 & 6.6758 \\
\hline & & 6-12 month & $-2.8753^{\circ}$ & 1.35212 & .035 & -5.5441 & -.2066 \\
\hline & & OA & $-6.4138^{\circ}$ & 2.16837 & .004 & -10.6937 & -2.1339 \\
\hline & OA & Healthy & $10.1163^{\circ}$ & 2.24474 & .000 & 5.6857 & 14.5469 \\
\hline & & 6-12 month & 3.5385 & 2.14425 & .101 & -.6938 & 7.7707 \\
\hline & & $2-10$ year & $6.4138^{\circ}$ & 2.16837 & .004 & 2.1339 & 10.6937 \\
\hline \multirow[t]{3}{*}{ Dunnett $t(2-\text { sided })^{b}$} & 6-12 month & Healthy & $6.5778^{\circ}$ & 1.47148 & .000 & 3.0837 & 10.0720 \\
\hline & $2-10$ year & Healthy & $3.7025^{\circ}$ & 1.50642 & .040 & .1254 & 7.2796 \\
\hline & OA & Healthy & $10.1163^{\circ}$ & 2.24474 & .000 & 4.7860 & 15.4466 \\
\hline
\end{tabular}


Table D15. Between limb comparisons of quadriceps active motor threshold per group

\begin{tabular}{|c|c|c|c|c|c|c|c|c|c|c|}
\hline \multirow{3}{*}{\multicolumn{2}{|c|}{ Group_Small_Stack }} & & \multicolumn{5}{|c|}{ Paired Differences } & \multirow{4}{*}{$\frac{t}{-.101}$} & \multirow{4}{*}{$\frac{\mathrm{df}}{18}$} & \multirow{4}{*}{$\begin{aligned} \begin{array}{r}\text { Sig. (2- } \\
\text { tailed) }\end{array} \\
.920\end{aligned}$} \\
\hline & & & \multirow{3}{*}{$\begin{array}{c}\text { Mean } \\
-.10526\end{array}$} & \multirow{3}{*}{$\begin{array}{c}\begin{array}{c}\text { Std. } \\
\text { Deviation }\end{array} \\
4.53253\end{array}$} & \multirow{3}{*}{$\begin{array}{c}\begin{array}{c}\text { Std. Error } \\
\text { Mean }\end{array} \\
1.03983\end{array}$} & \multicolumn{2}{|c|}{$\begin{array}{c}95 \% \text { Confidence Interval of } \\
\text { the Difference }\end{array}$} & & & \\
\hline & & & & & & Lower & Upper & & & \\
\hline Healthy & Pair 1 & $\begin{array}{l}\text { AMT_MSO_Inv - } \\
\text { AMT_MSO_Un }\end{array}$ & & & & -2.28987 & 2.07935 & & & \\
\hline 6-12 month & Pair 1 & $\begin{array}{l}\text { AMT_MSO_Inv- } \\
\text { AMT_MSO_Un }\end{array}$ & .75000 & 8.00403 & 1.41493 & -2.13576 & 3.63576 & .530 & 31 & .600 \\
\hline $2-10$ year & Pair 1 & $\begin{array}{l}\text { AMT_MSO_Inv - } \\
\text { AMT_MSO_Un }\end{array}$ & .48276 & 4.91078 & .91191 & -1.38520 & 2.35072 & .529 & 28 & .601 \\
\hline$O A$ & Pair 1 & $\begin{array}{l}\text { AMT_MSO_Inv- } \\
\text { AMT_MSO_Un }\end{array}$ & 2.50000 & 3.81725 & 1.34960 & -.69130 & 5.69130 & 1.852 & 7 & .106 \\
\hline
\end{tabular}

Figure D1. Quadriceps function limb symmetry for healthy $(\diamond)$, early ACL-R $(\boldsymbol{\square})$, late ACL-R $(\mathbf{O})$, and ACL-R with osteoarthritis $(\Delta)$. Point estimates represent mean values with associated $95 \%$ confidence intervals.

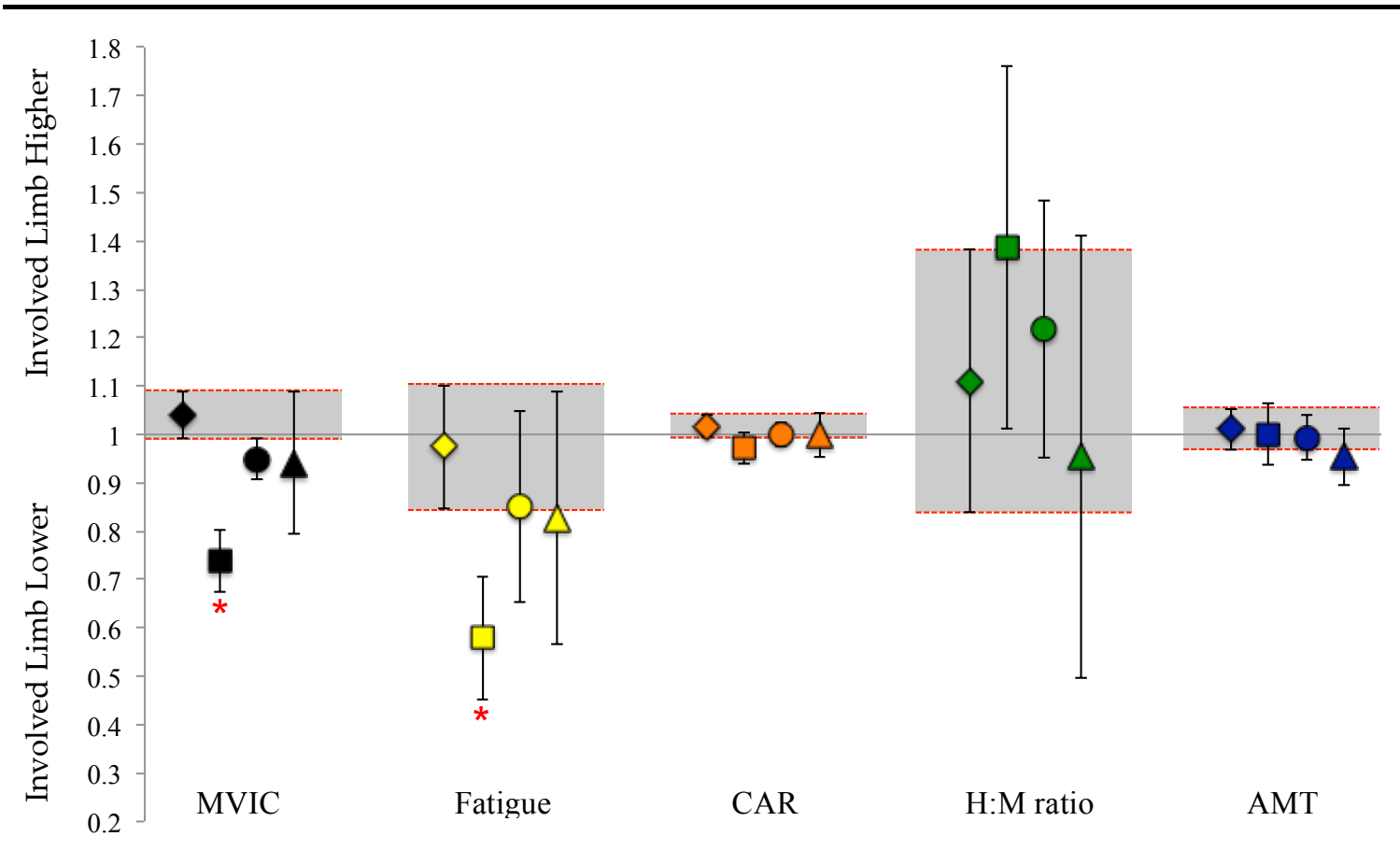


Figure D2. Cohen's $d$ effect sizes with 95\% confidence intervals comparing subjective outcomes in each ACL reconstructed group to healthy controls. Negative values indicate that ACL reconstructed patients reported worse values than healthy controls.

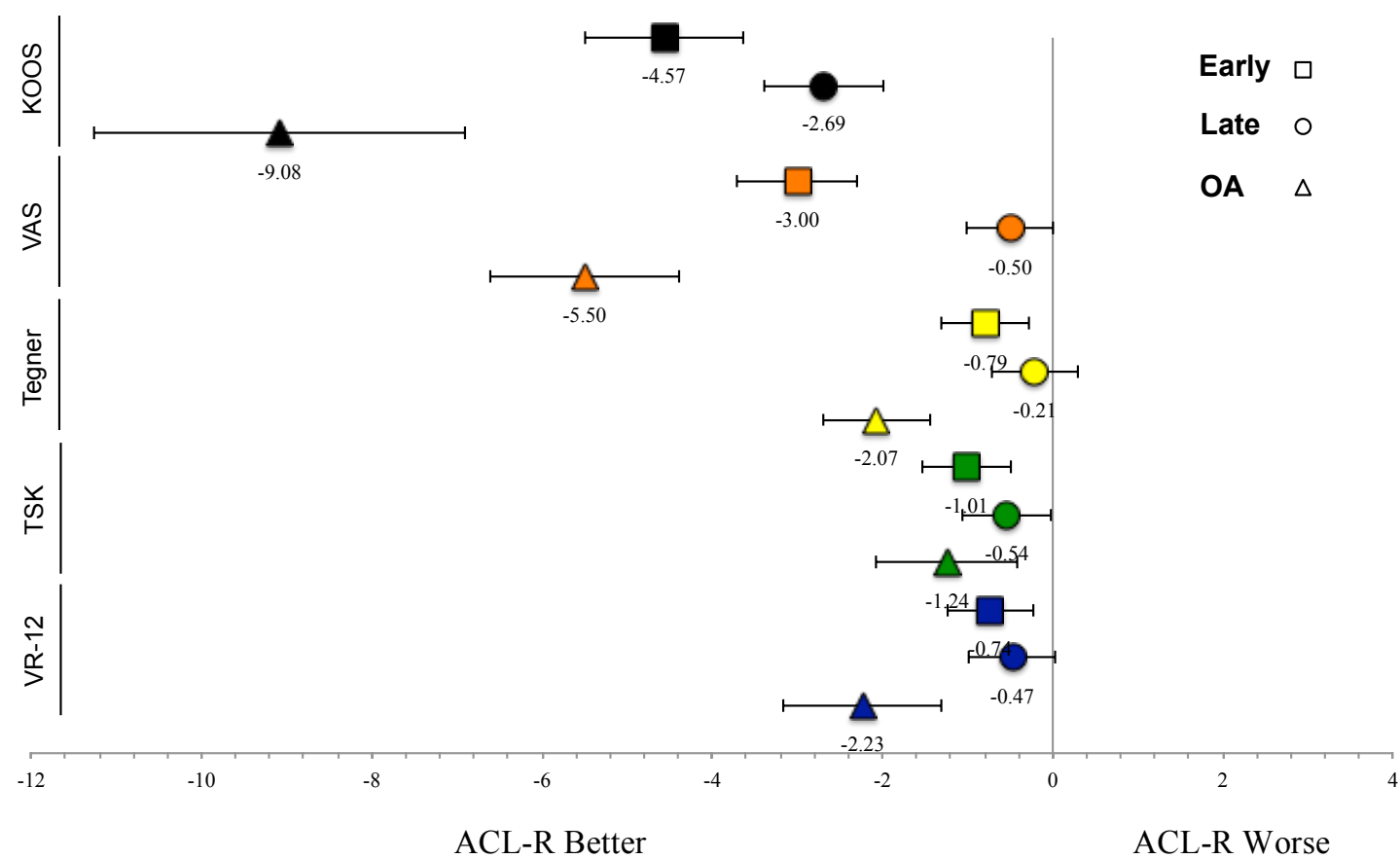


Figure D3. Pearson's $r$ or Spearman's $\rho$ correlation coefficients between measures of involved limb quadriceps function and time since surgery in ACL reconstructed patients
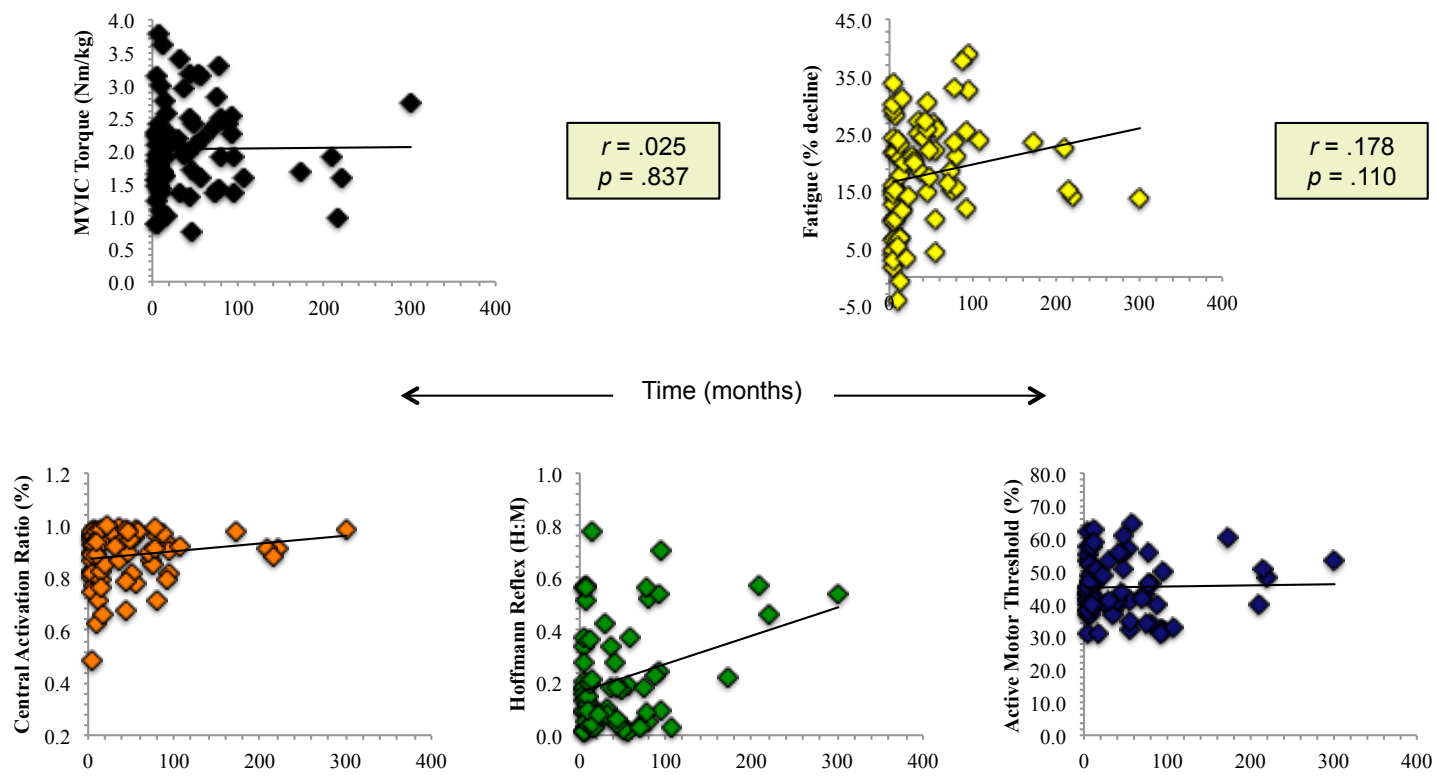

$$
\begin{aligned}
& \rho=.181 \\
& p=.128
\end{aligned}
$$$$
r=.326
$$

$$
\begin{aligned}
& r=.030 \\
& p=.806
\end{aligned}
$$

Figure D4. Pearson's $r$ or Spearman's $\rho$ correlation coefficients between measures of involved limb quadriceps function and age in ACL reconstructed patients
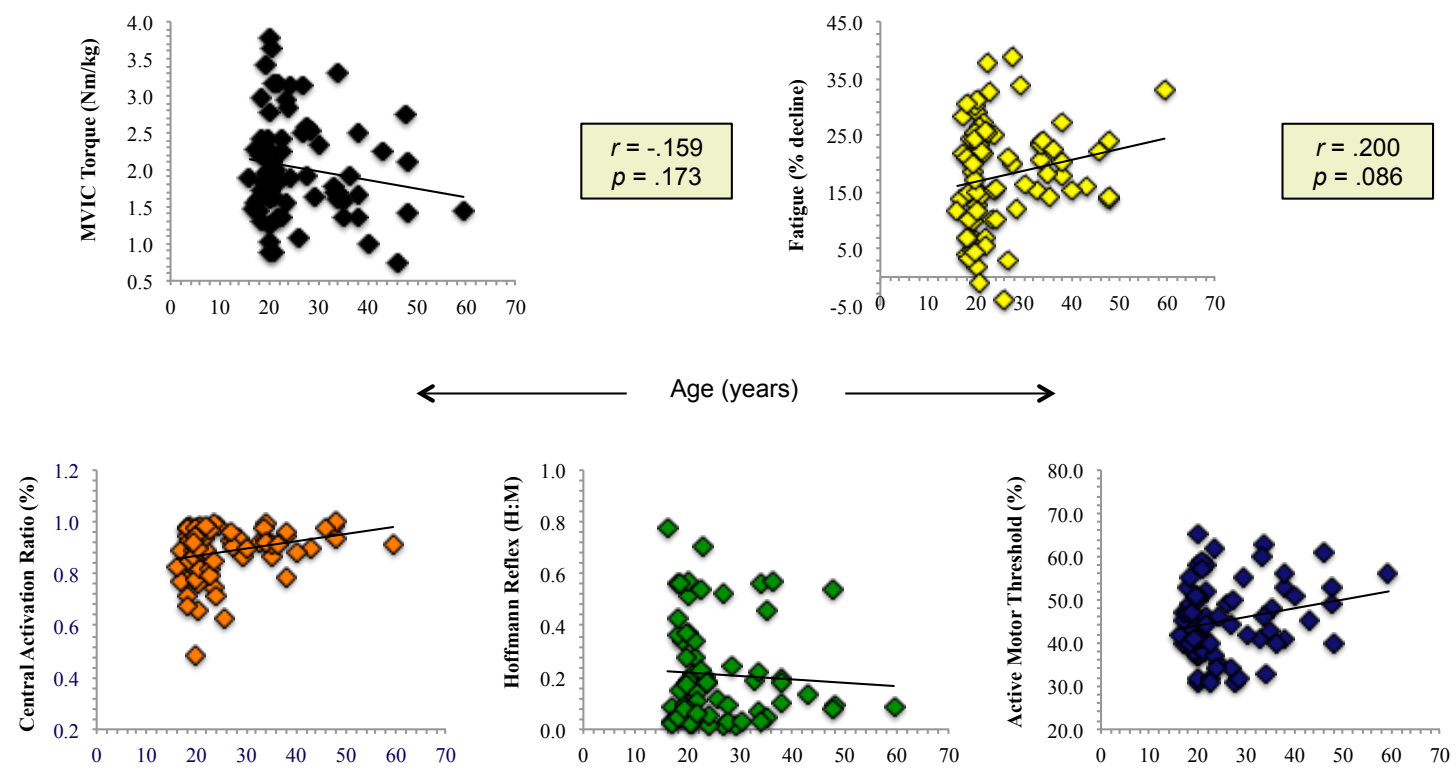

$$
\begin{aligned}
& \rho=.215 \\
& p=.064
\end{aligned}
$$

$$
r=-.062
$$$$
r=.212
$$$$
p=.072
$$ 
Figure D5. Independent $t$ tests comparing measures of involved limb quadriceps function between male and female ACL reconstructed patients

MVIC (Nm/kg)

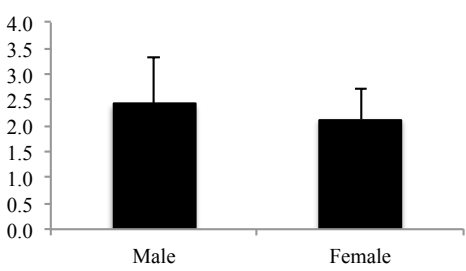

CAR (\%)

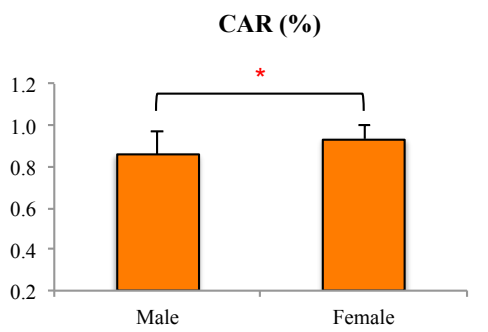

H:M ratio

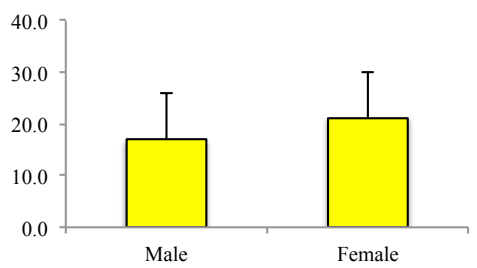

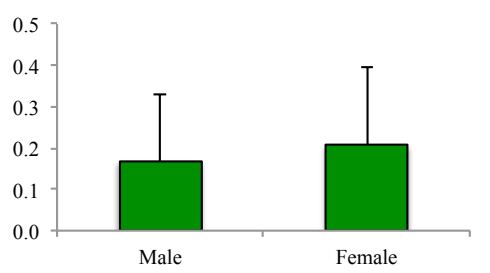

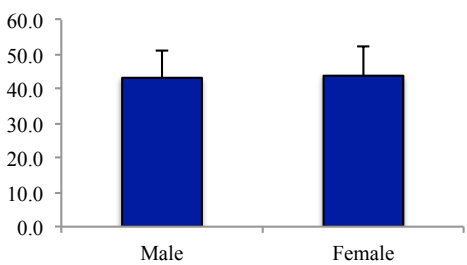

Figure D6. ANOVA comparison of graft type for measures of involved limb quadriceps function in ACL reconstructed patients
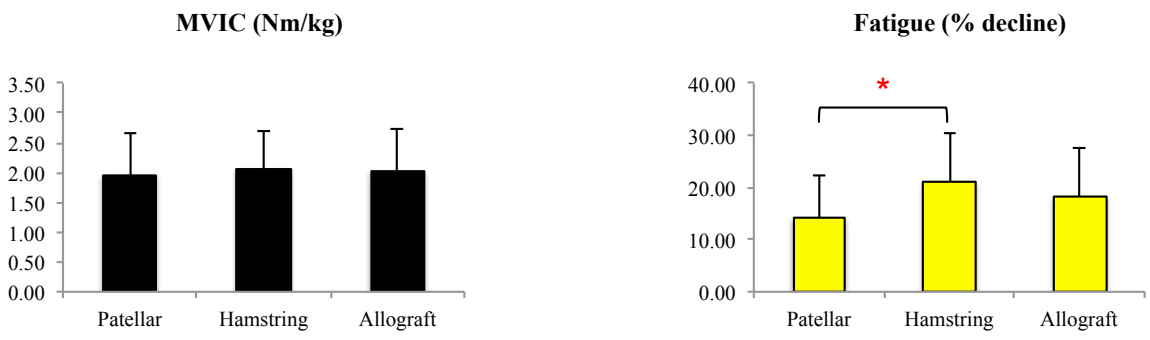

CAR (\%)

H:M ratio

AMT (\% 2T)
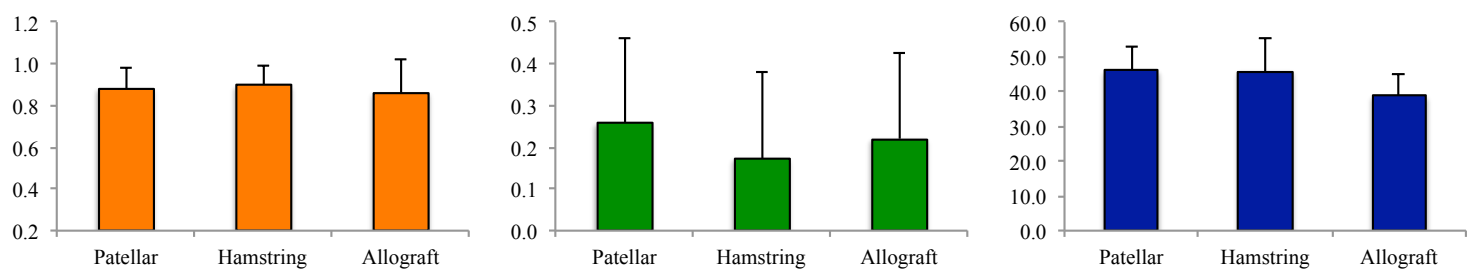


\section{MANUSCRIPT II}

Table D16. Multiple regression model summary to predict knee function (KOOS) in ACL reconstructed patients per group

\begin{tabular}{|c|c|c|c|c|c|c|c|c|c|c|}
\hline \multirow[b]{2}{*}{ Group_Small_Stack } & \multirow[b]{2}{*}{ Model } & \multirow[b]{2}{*}{$\mathbf{R}$} & \multirow[b]{2}{*}{ R Square } & \multirow[b]{2}{*}{$\begin{array}{c}\text { Adjusted R } \\
\text { Square }\end{array}$} & \multirow[b]{2}{*}{$\begin{array}{l}\text { Std. Error of } \\
\text { the Estimate }\end{array}$} & \multicolumn{5}{|c|}{ Change Statistics } \\
\hline & & & & & & $\begin{array}{c}\text { R Square } \\
\text { Change }\end{array}$ & F Change & df1 & $d f 2$ & Sig. F Change \\
\hline \multirow[t]{5}{*}{ 6-12 month } & 1 & $.627^{\mathrm{a}}$ & .393 & .374 & 7.34833 & .393 & 20.711 & 1 & 32 & .000 \\
\hline & 2 & $.760^{\mathrm{b}}$ & .578 & .551 & 6.22644 & .185 & 13.570 & 1 & 31 & .001 \\
\hline & 3 & $.813^{c}$ & .662 & .628 & 5.66695 & .084 & 7.423 & 1 & 30 & .011 \\
\hline & 4 & $.841^{\mathrm{d}}$ & .708 & .668 & 5.35390 & .046 & 4.611 & 1 & 29 & .040 \\
\hline & 5 & $.867^{\mathrm{e}}$ & .752 & .708 & 5.01759 & .044 & 5.018 & 1 & 28 & .033 \\
\hline $2-10$ year & 1 & $.445^{1}$ & .198 & .169 & 5.45797 & .198 & 6.899 & 1 & 28 & .014 \\
\hline \multirow[t]{2}{*}{$O A$} & 1 & $.771^{9}$ & .595 & .527 & 7.45126 & .595 & 8.806 & 1 & 6 & .025 \\
\hline & 2 & $.917^{\mathrm{h}}$ & .841 & .778 & 5.10887 & .246 & 7.763 & 1 & 5 & .039 \\
\hline
\end{tabular}

a. Predictors: (Constant), Norm_Work_Ext_90_Inv

b. Predictors: (Constant), Norm_Work_Ext_90_Inv, VAS_Inv

c. Predictors: (Constant), Norm_Work_Ext_90_Inv, VAS_Inv, Norm_Torque_Ext_90_Inv

d. Predictors: (Constant), Norm_Work_Ext_90_Inv, VAS_Inv, Norm_Torque_Ext_90_Inv, LSI_AMT

e. Predictors: (Constant), Norm_Work_Ext_90_Inv, VAS_Inv, Norm_Torque_Ext_90_Inv, LSI_AMT, Tegner_Current

f. Predictors: (Constant), LSI_Peak_Torque_Ext_90

g. Predictors: (Constant), Tampa

h. Predictors: (Constant), Tampa, Norm_Torque_Ext_90_Inv 
Table D17. ANOVA to predict knee function (KOOS) in ACL reconstructed patients per group

\begin{tabular}{|c|c|c|c|c|c|c|c|}
\hline Group_Small_Stack & Model & & $\begin{array}{l}\text { Sum of } \\
\text { Squares }\end{array}$ & df & Mean Square & $\mathrm{F}$ & Sig. \\
\hline \multirow[t]{15}{*}{$6-12$ month } & 1 & Regression & 1118.334 & 1 & 1118.334 & 20.711 & $.000^{\circ}$ \\
\hline & & Residual & 1727.933 & 32 & 53.998 & & \\
\hline & & Total & 2846.267 & 33 & & & \\
\hline & 2 & Regression & 1644.443 & 2 & 822.221 & 21.208 & $.000^{c}$ \\
\hline & & Residual & 1201.825 & 31 & 38.769 & & \\
\hline & & Total & 2846.267 & 33 & & & \\
\hline & 3 & Regression & 1882.836 & 3 & 627.612 & 19.543 & $.000^{\mathrm{d}}$ \\
\hline & & Residual & 963.431 & 30 & 32.114 & & \\
\hline & & Total & 2846.267 & 33 & & & \\
\hline & 4 & Regression & 2015.005 & 4 & 503.751 & 17.574 & $.000^{e}$ \\
\hline & & Residual & 831.262 & 29 & 28.664 & & \\
\hline & & Total & 2846.267 & 33 & & & \\
\hline & 5 & Regression & 2141.333 & 5 & 428.267 & 17.011 & $.000^{\top}$ \\
\hline & & Residual & 704.934 & 28 & 25.176 & & \\
\hline & & Total & 2846.267 & 33 & & & \\
\hline \multirow[t]{3}{*}{$2-10$ year } & 1 & Regression & 205.524 & 1 & 205.524 & 6.899 & $.014^{9}$ \\
\hline & & Residual & 834.104 & 28 & 29.789 & & \\
\hline & & Total & 1039.628 & 29 & & & \\
\hline \multirow[t]{6}{*}{$\mathrm{OA}$} & 1 & Regression & 488.912 & 1 & 488.912 & 8.806 & $.025^{n}$ \\
\hline & & Residual & 333.128 & 6 & 55.521 & & \\
\hline & & Total & 822.040 & 7 & & & \\
\hline & 2 & Regression & 691.537 & 2 & 345.769 & 13.248 & $.010^{\prime}$ \\
\hline & & Residual & 130.503 & 5 & 26.101 & & \\
\hline & & Total & 822.040 & 7 & & & \\
\hline
\end{tabular}

a. Dependent Variable: KOOS_Total

b. Predictors: (Constant), Norm_Work_Ext_90_Inv

c. Predictors: (Constant), Norm_Work_Ext_90_Inv, VAS_Inv

d. Predictors: (Constant), Norm_Work_Ext_90_Inv, VAS_Inv, Norm_Torque_Ext_90_Inv

e. Predictors: (Constant), Norm_Work_Ext_90_Inv, VAS_Inv, Norm_Torque_Ext_90_Inv, LSI_AMT

f. Predictors: (Constant), Norm_Work_Ext_90_Inv, VAS_Inv, Norm_Torque_Ext_90_Inv, LSI_AMT, Tegner_Current

g. Predictors: (Constant), LSI_Peak_Torque_Ext_90

h. Predictors: (Constant), Tampa

i. Predictors: (Constant), Tampa, Norm_Torque_Ext_90_Inv 
Table D18. Multiple regression coefficients to predict knee function (KOOS) in ACL reconstructed patients per group

\begin{tabular}{|c|c|c|c|c|c|c|c|c|c|}
\hline Group Small_Stack & Model & & \multicolumn{2}{|c|}{ Unstandardized Coefficients } & $\begin{array}{c}\begin{array}{c}\text { Standardized } \\
\text { Coefficients }\end{array} \\
\text { Beta }\end{array}$ & $\mathrm{t}$ & Sig. & \multicolumn{2}{|c|}{$\begin{array}{l}\text { 95.0\% Confidence Interval for } \\
\text { B }\end{array}$} \\
\hline \multirow[t]{14}{*}{$6-12$ month } & 1 & (Constant) & 66.090 & 4.867 & & 13.580 & .000 & 56.176 & 76.003 \\
\hline & 2 & (Constant) & 72.356 & 4.461 & & 16.220 & .000 & 63.258 & 81.454 \\
\hline & & Norm_Work_Ext_90_Inv & 1.304 & .283 & .546 & 4.601 & .000 & .726 & 1.882 \\
\hline & & VAS_Inv & -4.465 & 1.212 & -.437 & -3.684 & .001 & -6.937 & -1.993 \\
\hline & & VAS_Inv & -4.650 & 1.105 & -.456 & -4.207 & .000 & -6.907 & -2.393 \\
\hline & & $\begin{array}{l}\text { Norm_Torque_Ext_90_In } \\
\mathrm{v}\end{array}$ & -20.495 & 7.522 & -.987 & -2.725 & .011 & -35.857 & -5.132 \\
\hline & 4 & (Constant) & 84.960 & 6.781 & & 12.528 & .000 & 71.091 & 98.830 \\
\hline & & Norm_Work_Ext_90_Inv & 3.201 & .831 & 1.342 & 3.851 & .001 & 1.501 & 4.901 \\
\hline & & VAS_Inv & -4.142 & 1.071 & -.406 & -3.869 & .001 & -6.332 & -1.952 \\
\hline & & $\underset{\mathrm{v}}{\text { Norm_Torque_Ext_90_In }}$ & -17.920 & 7.207 & -.863 & -2.486 & .019 & -32.660 & -3.180 \\
\hline & & VAS_Inv & -4.359 & 1.008 & -.427 & -4.324 & .000 & -6.424 & -2.294 \\
\hline & & $\underset{v}{\text { Norm_Torque_Ext_90_In }}$ & -13.952 & 6.983 & -.672 & -1.998 & .056 & -28.255 & .352 \\
\hline & & LSI_AMT & -12.977 & 5.073 & -.255 & -2.558 & .016 & -23.369 & -2.586 \\
\hline & & Tegner_Current & 1.122 & .501 & .234 & 2.240 & .033 & .096 & 2.148 \\
\hline \multirow[t]{2}{*}{ 2-10 year } & 1 & (Constant) & 72.308 & 7.594 & & 9.522 & .000 & 56.752 & 87.864 \\
\hline & & $\begin{array}{l}\text { LSI_Peak_Torque_Ext_9 } \\
0\end{array}$ & 20.970 & 7.984 & .445 & 2.627 & .014 & 4.616 & 37.324 \\
\hline \multirow[t]{4}{*}{$\mathrm{OA}$} & 1 & (Constant) & 126.959 & 17.236 & & 7.366 & .000 & 84.784 & 169.134 \\
\hline & & Tampa & -1.404 & .473 & -.771 & -2.967 & .025 & -2.562 & -.246 \\
\hline & 2 & (Constant) & 99.127 & 15.474 & & 6.406 & .001 & 59.350 & 138.904 \\
\hline & & $\underset{v}{\text { Norm_Torque_Ext_90_In }}$ & 13.524 & 4.854 & .526 & 2.786 & .039 & 1.047 & 26.000 \\
\hline
\end{tabular}

a. Dependent Variable: KOOS_Total

Table D19. Multiple regression model summary to predict global health (VR-12) in ACL reconstructed patients per group

\begin{tabular}{|c|c|c|c|c|c|c|c|c|c|c|}
\hline Group Small Stack & Model & $\mathrm{R}$ & R Square & $\begin{array}{l}\text { Adjusted R } \\
\text { Square }\end{array}$ & $\begin{array}{l}\text { Std. Error of } \\
\text { the Estimate }\end{array}$ & $\begin{array}{c}\text { R Square } \\
\text { Change }\end{array}$ & F Change & df1 & $\mathrm{df2}$ & Sig. F Change \\
\hline \multirow[t]{3}{*}{$6-12$ month } & 1 & $.585^{a}$ & .342 & .322 & 8.23706 & .342 & 16.653 & 1 & 32 & .000 \\
\hline & 2 & $.675^{\mathrm{b}}$ & .456 & .421 & 7.61075 & .114 & 6.483 & 1 & 31 & .016 \\
\hline & 3 & $.757^{c}$ & .573 & .530 & 6.85845 & .116 & 8.174 & 1 & 30 & .008 \\
\hline \multirow[t]{2}{*}{$\overline{O A}$} & 1 & $.929^{\mathrm{d}}$ & .864 & .841 & 5.67072 & .864 & 37.961 & 1 & 6 & .001 \\
\hline & 2 & $.972^{\mathrm{e}}$ & .944 & .922 & 3.98033 & .080 & 7.178 & 1 & 5 & .044 \\
\hline
\end{tabular}

a. Predictors: (Constant), Norm_Work_Ext_90_Inv

b. Predictors: (Constant), Norm_Work_Ext_90_Inv, Tegner_Current

c. Predictors: (Constant), Norm_Work_Ext_90_Inv, Tegner_Current, VAS_Inv

d. Predictors: (Constant), Tegner_Current

e. Predictors: (Constant), Tegner_Current, VAS_Inv 
Table D20. ANOVA to predict global health (VR-12) in ACL reconstructed patients per group

\begin{tabular}{|c|c|c|c|c|c|c|c|}
\hline Group_Small_Stack & Model & & $\begin{array}{l}\text { Sum of } \\
\text { Squares }\end{array}$ & $\mathrm{df}$ & Mean Square & $\mathrm{F}$ & Sig. \\
\hline \multirow[t]{9}{*}{$6-12$ month } & 1 & Regression & 1129.876 & 1 & 1129.876 & 16.653 & $.000^{\circ}$ \\
\hline & & Residual & 2171.174 & 32 & 67.849 & & \\
\hline & & Total & 3301.049 & 33 & & & \\
\hline & 2 & Regression & 1505.420 & 2 & 752.710 & 12.995 & $.000^{c}$ \\
\hline & & Residual & 1795.629 & 31 & 57.924 & & \\
\hline & & Total & 3301.049 & 33 & & & \\
\hline & 3 & Regression & 1889.897 & 3 & 629.966 & 13.393 & $.000^{\mathrm{d}}$ \\
\hline & & Residual & 1411.152 & 30 & 47.038 & & \\
\hline & & Total & 3301.049 & 33 & & & \\
\hline \multirow[t]{6}{*}{ OA } & 1 & Regression & 1220.711 & 1 & 1220.711 & 37.961 & $.001^{e}$ \\
\hline & & Residual & 192.942 & 6 & 32.157 & & \\
\hline & & Total & 1413.653 & 7 & & & \\
\hline & 2 & Regression & 1334.438 & 2 & 667.219 & 42.114 & $.001^{1}$ \\
\hline & & Residual & 79.215 & 5 & 15.843 & & \\
\hline & & Total & 1413.653 & 7 & & & \\
\hline
\end{tabular}
a. Dependent Variable: VR_12
b. Predictors: (Constant), Norm_Work_Ext_90_Inv
c. Predictors: (Constant), Norm_Work_Ext_90_Inv, Tegner_Current
d. Predictors: (Constant), Norm_Work_Ext_90_Inv, Tegner_Current, VAS_Inv
e. Predictors: (Constant), Tegner_Current
f. Predictors: (Constant), Tegner_Current, VAS_Inv 
Table D21. Multiple regression coefficients to predict global health (VR-12) in ACL reconstructed patients per group

\begin{tabular}{|c|c|c|c|c|c|c|c|c|c|}
\hline Group_Small_Stack & Model & & \multicolumn{2}{|c|}{ Unstandardized Coefficients } & $\begin{array}{c}\begin{array}{c}\text { Standardized } \\
\text { Coefficients }\end{array} \\
\text { Beta } \\
\end{array}$ & $\mathrm{t}$ & Sig. & \multicolumn{2}{|c|}{$\begin{array}{c}95.0 \% \text { Confidence Interval for } \\
\text { B }\end{array}$} \\
\hline \multirow[t]{8}{*}{ 6-12 month } & 1 & (Constant) & 58.872 & 5.455 & & 10.792 & .000 & 47.760 & 69.985 \\
\hline & & Norm_Work_Ext_90_Inv & 1.504 & .368 & .585 & 4.081 & .000 & .753 & 2.254 \\
\hline & 2 & (Constant) & 51.969 & 5.724 & & 9.080 & .000 & 40.296 & 63.642 \\
\hline & & Norm_Work_Ext_90_Inv & 1.192 & .362 & .464 & 3.297 & .002 & .455 & 1.930 \\
\hline & & Tegner_Current & 1.847 & .725 & .358 & 2.546 & .016 & .368 & 3.326 \\
\hline & 3 & (Constant) & 56.411 & 5.387 & & 10.472 & .000 & 45.410 & 67.412 \\
\hline & & Tegner_Current & 2.105 & .660 & .408 & 3.190 & .003 & .758 & 3.453 \\
\hline & & VAS_Inv & -3.853 & 1.348 & -.351 & -2.859 & .008 & -6.606 & -1.101 \\
\hline \multirow[t]{4}{*}{ OA } & 1 & (Constant) & 35.301 & 5.814 & & 6.071 & .001 & 21.074 & 49.528 \\
\hline & & Tegner_Current & 7.912 & 1.284 & .929 & 6.161 & .001 & 4.770 & 11.054 \\
\hline & 2 & (Constant) & 24.553 & 5.723 & & 4.291 & .008 & 9.843 & 39.264 \\
\hline & & Tegner_Current & 8.861 & .969 & 1.041 & 9.149 & .000 & 6.372 & 11.351 \\
\hline
\end{tabular}

a. Dependent Variable: VR_12

Figure D7. Pearson's $r$ correlation coefficients between total KOOS score and pain at rest, kinesiophobia, and activity level in patients early after ACL reconstruction
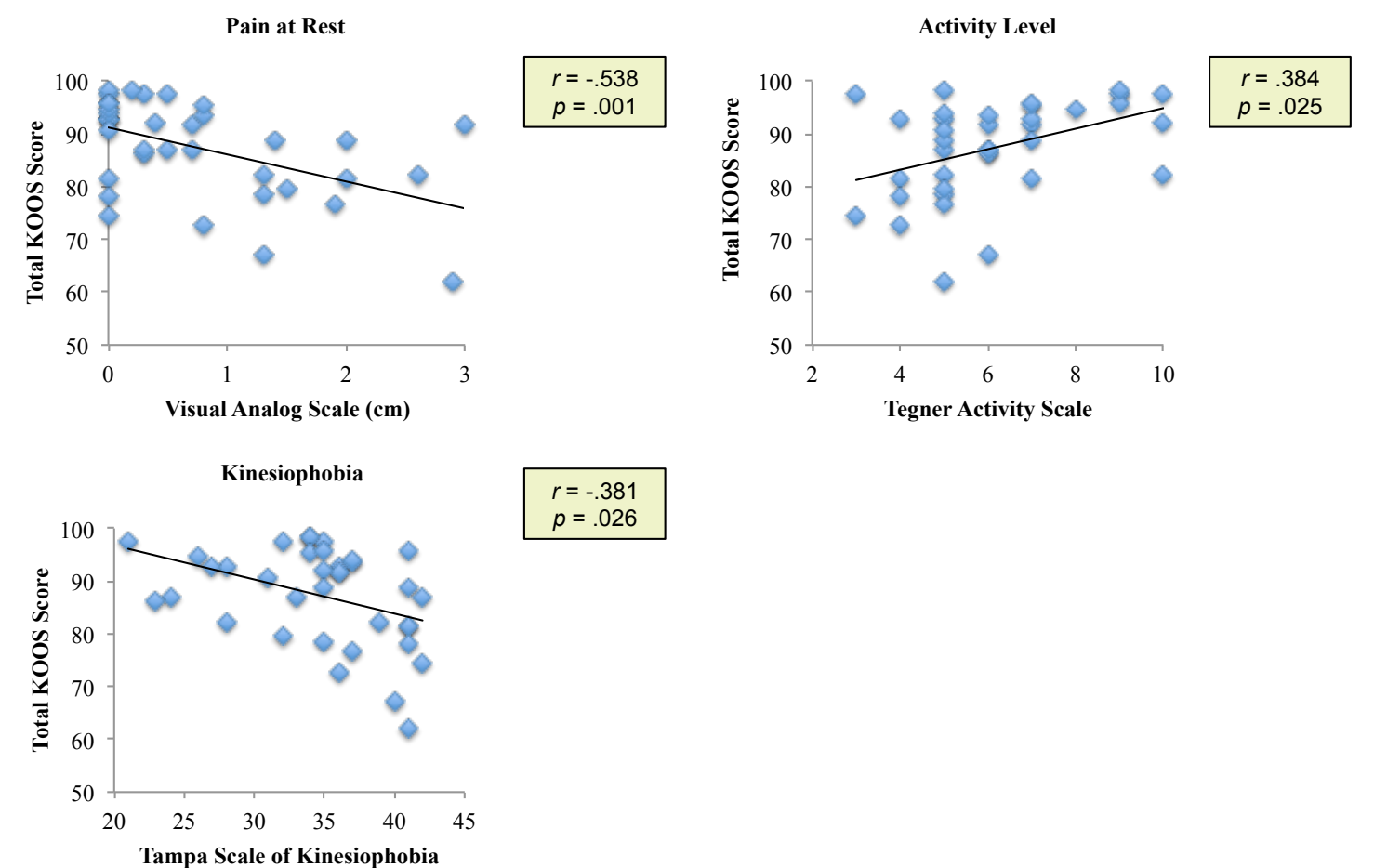
Figure D8. Pearson's $r$ correlation coefficients between total VR-12 score and activity level, pain at rest, and time since surgery in patients early after ACL reconstruction
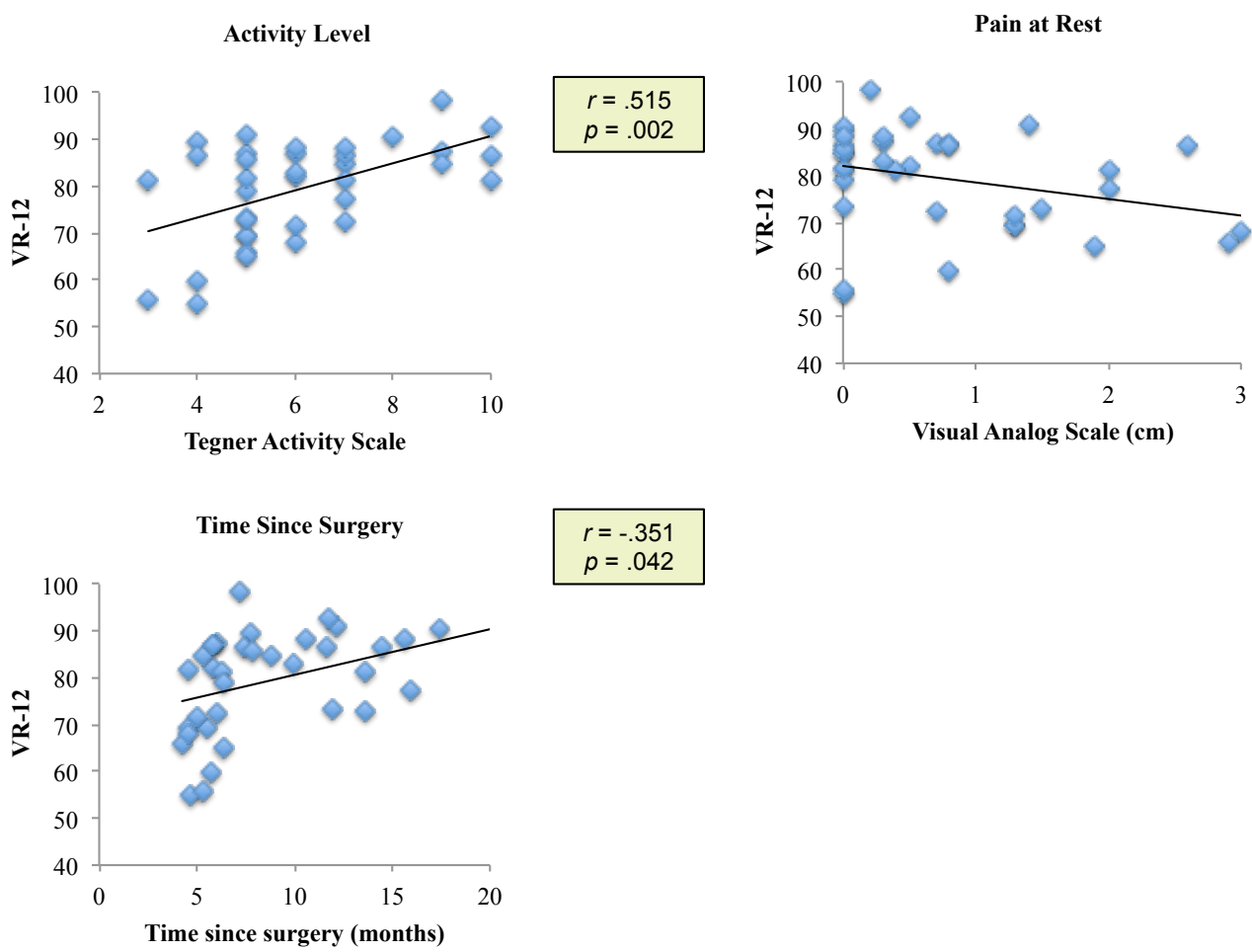

Figure D9. Pearson's $r$ correlation coefficients between total KOOS score and age in patients late after ACL reconstruction

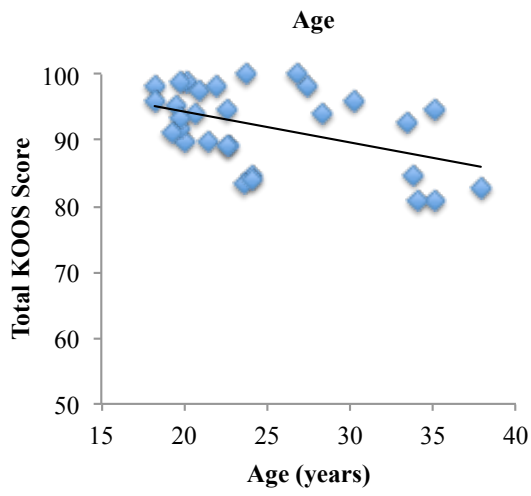


Figure D10. Normalized knee extension MVIC torque for the involved and uninvolved limbs

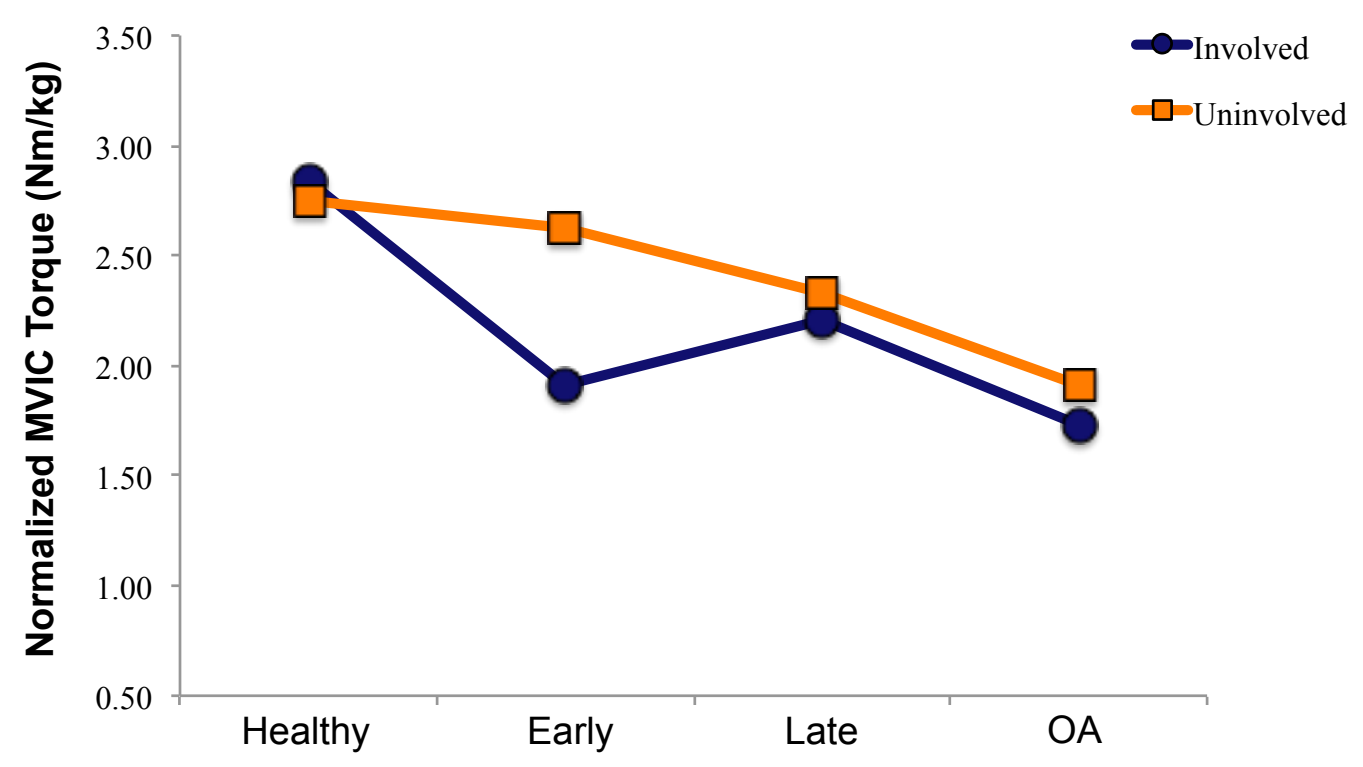

\section{MANUSCRIPT III}

Table D22. Paired $t$ tests comparing the involved to uninvolved limb among ACL reconstructed patients

\begin{tabular}{|c|c|c|c|c|c|c|c|c|c|}
\hline & & \multicolumn{5}{|c|}{ Paired Differences } & \multirow[b]{3}{*}{$t$} & \multirow[b]{3}{*}{$\mathrm{df}$} & \multirow{3}{*}{$\begin{array}{l}\text { Sig. (2- } \\
\text { tailed) }\end{array}$} \\
\hline & & \multirow[b]{2}{*}{ Mean } & \multirow{2}{*}{$\begin{array}{c}\text { Std. } \\
\text { Deviation }\end{array}$} & \multirow{2}{*}{$\begin{array}{l}\text { Std. Error } \\
\text { Mean }\end{array}$} & \multicolumn{2}{|c|}{$\begin{array}{l}95 \% \text { Confidence Interval of } \\
\text { the Difference }\end{array}$} & & & \\
\hline & & & & & Lower & Upper & & & \\
\hline Pair 1 & $\begin{array}{l}\text { Norm_Torque_Ext_90_In } \\
v-\text { - } \\
\text { Norm_Torque_Ext_90_u } \\
n\end{array}$ & -.38646 & .44967 & .05299 & -.49212 & -.28079 & -7.292 & 71 & .000 \\
\hline Pair 2 & $\begin{array}{l}\text { Norm_Torque_Ext_180_ } \\
\text { Inv - } \\
\text { Norm_Torque_Ext_180_ } \\
\text { Un }\end{array}$ & -.27746 & .29404 & .03465 & -.34655 & -.20836 & -8.007 & 71 & .000 \\
\hline Pair 3 & $\begin{array}{l}\text { Norm_Work_Ext_90_Inv } \\
\overline{-} \\
\text { Norm_Work_Ext_90_Un }\end{array}$ & -3.35121 & 3.32211 & .39151 & -4.13187 & -2.57056 & -8.560 & 71 & .000 \\
\hline Pair 4 & $\begin{array}{l}\text { Norm_Work_Ext_180_In } \\
\mathrm{v}- \\
\text { Norm_Work_Ext_180_Un }\end{array}$ & -2.39828 & 2.53354 & .29858 & -2.99363 & -1.80293 & -8.032 & 71 & .000 \\
\hline Pair 5 & $\begin{array}{l}\text { Norm_Power_Ext_90_Inv } \\
\overline{\text { Norm_Power_Ext_90_Un }}\end{array}$ & -.35730 & .38387 & .04524 & -.44750 & -.26710 & -7.898 & 71 & .000 \\
\hline Pair 6 & $\begin{array}{l}\text { Norm_Power_Ext_180_I } \\
\text { nv - } \\
\text { Norm_Power_Ext_180_U } \\
n\end{array}$ & -.43173 & .49010 & .05776 & -.54689 & -.31656 & -7.475 & 71 & .000 \\
\hline Pair 7 & $\begin{array}{l}\text { Norm_MVIC_Torque_Inv } \\
\overline{\text { Norm_MVIC_Torque_Un }}\end{array}$ & -.40881 & .53436 & .06298 & -.53438 & -.28324 & -6.492 & 71 & .000 \\
\hline Pair 8 & Fatigue_Inv - Fatigue_Un & -1.98139 & 7.96471 & .93865 & -3.85301 & -.10977 & -2.111 & 71 & .038 \\
\hline Pair 9 & CAR_Inv - CAR_Un & -.01470 & .07184 & .00847 & -.03159 & .00218 & -1.737 & 71 & .087 \\
\hline Pair 10 & HM_Inv - HM_Un & .02107 & .11356 & .01398 & -.00684 & .04899 & 1.507 & 65 & .137 \\
\hline Pair 11 & $\begin{array}{l}\text { AMT_MSO_Inv - } \\
\text { AMT_MSO_Un }\end{array}$ & .84058 & 6.40456 & .77102 & -.69796 & 2.37912 & 1.090 & 68 & .279 \\
\hline
\end{tabular}


Table D23. Paired $t$ tests comparing the matched 'involved' to matched 'uninvolved' limb among healthy controls

\begin{tabular}{|c|c|c|c|c|c|c|c|c|c|}
\hline & & \multicolumn{5}{|c|}{ Paired Differences } & \multirow[b]{3}{*}{$t$} & \multirow[b]{3}{*}{$\mathrm{df}$} & \multirow{3}{*}{$\begin{array}{l}\text { Sig. (2- } \\
\text { tailed) }\end{array}$} \\
\hline & & \multirow[b]{2}{*}{ Mean } & \multirow{2}{*}{$\begin{array}{c}\text { Std. } \\
\text { Deviation }\end{array}$} & \multirow{2}{*}{$\begin{array}{l}\text { Std. Error } \\
\text { Mean }\end{array}$} & \multicolumn{2}{|c|}{$\begin{array}{l}95 \% \text { Confidence Interval of } \\
\text { the Difference }\end{array}$} & & & \\
\hline & & & & & Lower & Upper & & & \\
\hline Pair 2 & $\begin{array}{l}\text { Norm_Torque_Ext_180_ } \\
\text { Inv - } \\
\text { Norm_Torque_Ext_180_ } \\
\text { Un }\end{array}$ & .01105 & .20665 & .03773 & -.06612 & .08821 & .293 & 29 & .772 \\
\hline Pair 3 & $\begin{array}{l}\text { Norm_Work_Ext_90_Inv } \\
- \\
\text { Norm_Work_Ext_90_Un }\end{array}$ & -.39151 & 2.74990 & .50206 & -1.41834 & .63532 & -.780 & 29 & .442 \\
\hline Pair 4 & $\begin{array}{l}\text { Norm_Work_Ext_180_In } \\
\text { v- } \\
\text { Norm_Work_Ext_180_Un }\end{array}$ & -.06395 & 2.19357 & .40049 & -.88304 & .75514 & -.160 & 29 & .874 \\
\hline Pair 5 & $\begin{array}{l}\text { Norm_Power_Ext_90_Inv } \\
\overline{-} \\
\text { Norm_Power_Ext_90_Un }\end{array}$ & -.04185 & .32865 & .06000 & -.16457 & .08087 & -.697 & 29 & .491 \\
\hline Pair 7 & $\begin{array}{l}\text { Norm_MVIC_Torque_Inv } \\
\overline{\text { Norm_MVIC_Torque_Un }}\end{array}$ & .08702 & .34571 & .06312 & -.04206 & .21611 & 1.379 & 29 & .179 \\
\hline Pair 8 & Fatigue_Inv - Fatigue_Un & -.41663 & 6.22494 & 1.15594 & -2.78447 & 1.95121 & -.360 & 28 & .721 \\
\hline Pair 9 & CAR_Inv - CAR_Un & .01331 & .05220 & .00969 & -.00654 & .03317 & 1.373 & 28 & .181 \\
\hline Pair 10 & HM_Inv - HM_Un & -.00028 & .08727 & .01820 & -.03802 & .03746 & -.015 & 22 & .988 \\
\hline Pair 11 & $\begin{array}{l}\text { AMT_MSO_Inv - } \\
\text { AMT_MSO_Un }\end{array}$ & -.10526 & 4.53253 & 1.03983 & -2.28987 & 2.07935 & -.101 & 18 & .920 \\
\hline
\end{tabular}

Table D24. Independent $t$ tests comparing the involved ACL reconstructed limb to matched healthy limb

\begin{tabular}{|c|c|c|c|c|c|c|c|c|c|c|}
\hline & & \multicolumn{2}{|c|}{$\begin{array}{l}\text { Levene's Test for Equality of } \\
\text { Variances }\end{array}$} & \multicolumn{7}{|c|}{ t-test for Equality of Means } \\
\hline & & \multirow[b]{2}{*}{$\mathrm{F}$} & \multirow[b]{2}{*}{ Sig. } & \multirow[b]{2}{*}{$\mathrm{t}$} & \multirow[b]{2}{*}{ df } & \multirow{2}{*}{$\begin{array}{l}\text { Sig. (2- } \\
\text { tailed) }\end{array}$} & \multirow{2}{*}{$\begin{array}{c}\text { Mean } \\
\text { Difference }\end{array}$} & \multirow{2}{*}{$\begin{array}{l}\text { Std. Error } \\
\text { Difference }\end{array}$} & \multicolumn{2}{|c|}{$\begin{array}{l}95 \% \text { Confidence Interval of } \\
\text { the Difference }\end{array}$} \\
\hline & & & & & & & & & & \\
\hline \multirow[t]{2}{*}{$\begin{array}{l}\text { Norm_Torque_Ext_90_In } \\
\mathrm{v}\end{array}$} & $\begin{array}{l}\text { Equal variances } \\
\text { assumed }\end{array}$ & 1.282 & .260 & 5.686 & 100 & .000 & .58165 & .10229 & .37870 & .78459 \\
\hline & $\begin{array}{l}\text { Equal variances not } \\
\text { assumed }\end{array}$ & & & 6.178 & 65.984 & .000 & .58165 & .09415 & .39367 & .76962 \\
\hline \multirow[t]{2}{*}{$\begin{array}{l}\text { Norm_Torque_Ext_180_ } \\
\text { Inv }\end{array}$} & $\begin{array}{l}\text { Equal variances } \\
\text { assumed }\end{array}$ & .159 & .691 & 5.486 & 100 & .000 & .42667 & .07778 & .27236 & .58098 \\
\hline & $\begin{array}{l}\text { Equal variances not } \\
\text { assumed }\end{array}$ & & & 5.652 & 58.131 & .000 & .42667 & .07549 & .27558 & .57777 \\
\hline \multirow[t]{2}{*}{ Norm_Work_Ext_90_Inv } & $\begin{array}{l}\text { Equal variances } \\
\text { assumed }\end{array}$ & 2.600 & .110 & 6.026 & 100 & .000 & 5.47563 & .90865 & 3.67290 & 7.27837 \\
\hline & $\begin{array}{l}\text { Equal variances not } \\
\text { assumed }\end{array}$ & & & 6.722 & 70.431 & .000 & 5.47563 & .81464 & 3.85106 & 7.10020 \\
\hline \multirow[t]{2}{*}{$\begin{array}{l}\text { Norm_Work_Ext_180_In } \\
\mathrm{v}\end{array}$} & $\begin{array}{l}\text { Equal variances } \\
\text { assumed }\end{array}$ & 1.813 & .181 & 5.816 & 100 & .000 & 4.20915 & .72367 & 2.77341 & 5.64490 \\
\hline & $\begin{array}{l}\text { Equal variances not } \\
\text { assumed }\end{array}$ & & & 6.243 & 64.055 & .000 & 4.20915 & .67419 & 2.86233 & 5.55598 \\
\hline \multirow[t]{2}{*}{ Norm_Power_Ext_90_Inv } & $\begin{array}{l}\text { Equal variances } \\
\text { assumed }\end{array}$ & .400 & .529 & 5.559 & 100 & .000 & .55268 & .09943 & .35542 & .74994 \\
\hline & $\begin{array}{l}\text { Equal variances not } \\
\text { assumed }\end{array}$ & & & 5.778 & 59.325 & .000 & .55268 & .09565 & .36130 & .74406 \\
\hline \multirow[t]{2}{*}{$\begin{array}{l}\text { Norm_Power_Ext_180_I } \\
\mathrm{nv}\end{array}$} & $\begin{array}{l}\text { Equal variances } \\
\text { assumed }\end{array}$ & .295 & .588 & 5.436 & 100 & .000 & .75554 & .13898 & .47981 & 1.03126 \\
\hline & $\begin{array}{l}\text { Equal variances not } \\
\text { assumed }\end{array}$ & & & 5.558 & 57.094 & .000 & .75554 & .13594 & .48333 & 1.02775 \\
\hline \multirow[t]{2}{*}{ Norm_MVIC_Torque_Inv } & $\begin{array}{l}\text { Equal variances } \\
\text { assumed }\end{array}$ & .005 & .941 & 5.865 & 100 & .000 & .81958 & .13973 & .54235 & 1.09681 \\
\hline & $\begin{array}{l}\text { Equal variances not } \\
\text { assumed }\end{array}$ & & & 5.933 & 55.751 & .000 & .81958 & .13813 & .54284 & 1.09632 \\
\hline \multirow[t]{2}{*}{ Fatigue_Inv } & $\begin{array}{l}\text { Equal variances } \\
\text { assumed }\end{array}$ & 1.077 & .302 & 1.935 & 100 & .056 & 3.74540 & 1.93570 & -.09498 & 7.58578 \\
\hline & $\begin{array}{l}\text { Equal variances not } \\
\text { assumed }\end{array}$ & & & 2.050 & 62.073 & .045 & 3.74540 & 1.82697 & .09343 & 7.39738 \\
\hline \multirow[t]{2}{*}{ CAR_Inv } & $\begin{array}{l}\text { Equal variances } \\
\text { assumed }\end{array}$ & 9.752 & .002 & 3.426 & 99 & .001 & .06818 & .01990 & .02869 & .10767 \\
\hline & $\begin{array}{l}\text { Equal variances not } \\
\text { assumed }\end{array}$ & & & 4.321 & 89.241 & .000 & .06818 & .01578 & .03683 & .09954 \\
\hline \multirow[t]{2}{*}{ HM_Inv } & $\begin{array}{l}\text { Equal variances } \\
\text { assumed }\end{array}$ & 5.435 & .022 & -1.647 & 91 & .103 & -.06878 & .04177 & -.15175 & .01418 \\
\hline & $\begin{array}{l}\text { Equal variances not } \\
\text { assumed }\end{array}$ & & & -2.019 & 68.014 & .047 & -.06878 & .03407 & -.13678 & -.00079 \\
\hline \multirow[t]{2}{*}{ AMT_MSO_Inv } & $\begin{array}{l}\text { Equal variances } \\
\text { assumed }\end{array}$ & 11.456 & .001 & -3.167 & 89 & .002 & -6.15714 & 1.94401 & -10.01984 & -2.29444 \\
\hline & $\begin{array}{l}\text { Equal variances not } \\
\text { assumed }\end{array}$ & & & -4.510 & 71.081 & .000 & -6.15714 & 1.36530 & -8.87941 & -3.43488 \\
\hline
\end{tabular}


Table D25. Independent $t$ tests comparing the uninvolved ACL reconstructed limb to matched healthy limb

\begin{tabular}{|c|c|c|c|c|c|c|c|c|c|c|}
\hline & & \multicolumn{2}{|c|}{$\begin{array}{l}\text { Levene's Test for Equality of } \\
\text { Variances }\end{array}$} & \multicolumn{7}{|c|}{ t-test for Equality of Means } \\
\hline & & $\mathrm{F}$ & Sig. & $t$ & df & $\begin{array}{l}\text { Sig. (2- } \\
\text { tailed) }\end{array}$ & $\begin{array}{c}\text { Mean } \\
\text { Difference }\end{array}$ & $\begin{array}{l}\text { Std. Error } \\
\text { Difference }\end{array}$ & Lower & Upper \\
\hline $\begin{array}{l}\begin{array}{l}\text { Norm_Torque_Ext_90_U } \\
n\end{array} \\
\text { n }\end{array}$ & $\begin{array}{l}\text { Equal variances not } \\
\text { assumed }\end{array}$ & & & 1.491 & 45.190 & .143 & .17542 & .11764 & -.06149 & .41233 \\
\hline $\begin{array}{l}\text { Norm_Torque_Ext_180_ } \\
\text { Un }\end{array}$ & $\begin{array}{l}\text { Equal variances } \\
\text { assumed }\end{array}$ & .163 & .687 & 1.732 & 100 & .086 & .13816 & .07976 & -.02007 & .29640 \\
\hline Norm_Work_Ext_90_Un & $\begin{array}{l}\text { Equal variances not } \\
\text { assumed }\end{array}$ & & & 2.956 & 66.947 & .004 & 2.51592 & .85120 & .81690 & 4.21495 \\
\hline \multirow[t]{2}{*}{ Norm_Work_Ext_180_Un } & $\begin{array}{l}\text { Equal variances } \\
\text { assumed }\end{array}$ & .724 & .397 & 2.411 & 100 & .018 & 1.87482 & .77763 & .33203 & 3.41761 \\
\hline & $\begin{array}{l}\text { Equal variances not } \\
\text { assumed }\end{array}$ & & & 2.573 & 63.165 & .012 & 1.87482 & .72865 & .41881 & 3.33084 \\
\hline Norm_Power_Ext_90_Un & $\begin{array}{l}\text { Equal variances } \\
\text { assumed }\end{array}$ & .622 & .432 & 2.402 & 100 & .018 & .23723 & .09878 & .04126 & .43320 \\
\hline \multirow[t]{2}{*}{ Norm_MVIC_Torque_Un } & $\begin{array}{l}\text { Equal variances } \\
\text { assumed }\end{array}$ & .460 & .499 & 2.188 & 100 & .031 & .32374 & .14795 & .03022 & .61727 \\
\hline & $\begin{array}{l}\text { Equal variances not } \\
\text { assumed }\end{array}$ & & & 2.307 & 61.349 & .024 & .32374 & .14032 & .04318 & .60430 \\
\hline \multirow[t]{2}{*}{ Fatigue_Un } & $\begin{array}{l}\text { Equal variances } \\
\text { assumed }\end{array}$ & .295 & .589 & 1.123 & 99 & .264 & 1.97347 & 1.75673 & -1.51226 & 5.45920 \\
\hline & $\begin{array}{l}\text { Equal variances not } \\
\text { assumed }\end{array}$ & & & 1.108 & 50.315 & .273 & 1.97347 & 1.78117 & -1.60356 & 5.55050 \\
\hline \multirow[t]{2}{*}{ CAR_Un } & $\begin{array}{l}\text { Equal variances } \\
\text { assumed }\end{array}$ & 5.228 & .024 & 2.059 & 99 & .042 & .04017 & .01951 & .00146 & .07887 \\
\hline & $\begin{array}{l}\text { Equal variances not } \\
\text { assumed }\end{array}$ & & & 2.400 & 74.335 & .019 & .04017 & .01674 & .00682 & .07351 \\
\hline \multirow[t]{2}{*}{ HM_Un } & $\begin{array}{l}\text { Equal variances } \\
\text { assumed }\end{array}$ & 2.817 & .097 & -1.018 & 89 & .311 & -.04019 & .03949 & -.11865 & .03827 \\
\hline & $\begin{array}{l}\text { Equal variances not } \\
\text { assumed }\end{array}$ & & & -1.205 & 53.863 & .233 & -.04019 & .03335 & -.10707 & .02668 \\
\hline \multirow[t]{2}{*}{ AMT_MSO_Un } & $\begin{array}{l}\text { Equal variances } \\
\text { assumed }\end{array}$ & 12.828 & .001 & -2.905 & 90 & .005 & -5.24638 & 1.80591 & -8.83412 & -1.65863 \\
\hline & $\begin{array}{l}\text { Equal variances not } \\
\text { assumed }\end{array}$ & & & -4.263 & 87.144 & .000 & -5.24638 & 1.23066 & -7.69239 & -2.80036 \\
\hline
\end{tabular}

Table D26. Independent $t$ tests comparing limb symmetry indices between ACL reconstructed patients and healthy controls

\begin{tabular}{|c|c|c|c|c|c|c|c|c|c|c|}
\hline & & \multicolumn{2}{|c|}{$\begin{array}{l}\text { Levene's Test for Equality of } \\
\text { Variances }\end{array}$} & \multicolumn{7}{|c|}{ t-test for Equality of Means } \\
\hline & & \multirow[t]{2}{*}{ 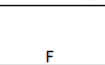 } & \multirow[b]{2}{*}{ Sig. } & \multirow[b]{2}{*}{$t$} & \multirow[b]{2}{*}{ df } & \multirow{2}{*}{$\begin{array}{l}\text { Sig. (2- } \\
\text { tailed) }\end{array}$} & \multirow{2}{*}{$\begin{array}{c}\text { Mean } \\
\text { Difference }\end{array}$} & \multirow{2}{*}{$\begin{array}{l}\text { Std. Error } \\
\text { Difference }\end{array}$} & \multicolumn{2}{|c|}{$\begin{array}{l}95 \% \text { Confidence Interval of } \\
\text { the Difference }\end{array}$} \\
\hline & & & & & & & & & Lower & Upper \\
\hline \multirow{2}{*}{${ }_{0}^{\text {LSIPeak_Torque_Ext_9 }}$} & $\begin{array}{l}\text { Equal variances } \\
\text { assumed }\end{array}$ & 6.359 & .013 & 2.410 & 100 & .018 & .47080 & .19538 & .08317 & .85843 \\
\hline & $\begin{array}{l}\text { Equal variances not } \\
\text { assumed }\end{array}$ & & & 1.565 & 29.326 & .128 & .47080 & .30077 & -.14404 & 1.08564 \\
\hline \multirow[t]{2}{*}{$\begin{array}{l}{ }_{80} \text { LI_Peak_Torque_Ext_1 } \\
\text { - }\end{array}$} & $\begin{array}{l}\text { Equal variances } \\
\text { assumed }\end{array}$ & 1.007 & .318 & 5.232 & 100 & .000 & .17893 & .03420 & .11108 & .24678 \\
\hline & $\begin{array}{l}\text { Equal variances not } \\
\text { assumed }\end{array}$ & & & 5.418 & 58.799 & .000 & .17893 & .03303 & .11284 & .24502 \\
\hline LSI_TotalWork_Ext_90 & $\begin{array}{l}\text { Equal variances not } \\
\text { assumed }\end{array}$ & & & 4.570 & 50.191 & .000 & .17494 & .03828 & .09806 & .25182 \\
\hline \multirow[t]{2}{*}{ LSI_TotalWork_Ext_180 } & $\begin{array}{l}\text { Equal variances } \\
\text { assumed }\end{array}$ & .045 & .833 & 4.533 & 100 & .000 & .17795 & .03926 & .10007 & .25584 \\
\hline & $\begin{array}{l}\text { Equal variances not } \\
\text { assumed }\end{array}$ & & & 3.958 & 41.884 & .000 & .17795 & .04496 & .08721 & .26869 \\
\hline \multirow[t]{2}{*}{ LSI_Avg_Power_Ext_90 } & $\begin{array}{l}\text { Equal variances } \\
\text { assumed }\end{array}$ & .159 & .691 & 4.241 & 100 & .000 & .16878 & .03979 & .08983 & .24773 \\
\hline & $\begin{array}{l}\text { Equal variances not } \\
\text { assumed }\end{array}$ & & & 3.816 & 44.082 & .000 & .16878 & .04423 & .07964 & .25792 \\
\hline LSI_MVIC & $\begin{array}{l}\text { Equal variances not } \\
\text { assumed }\end{array}$ & & & 5.558 & 77.169 & .000 & .18939 & .03408 & .12154 & .25724 \\
\hline \multirow[t]{2}{*}{ LSI_Fatigue_Diff } & $\begin{array}{l}\text { Equal variances } \\
\text { assumed }\end{array}$ & .733 & .394 & -.740 & 99 & .461 & -1.22093 & 1.65078 & -4.49643 & 2.05457 \\
\hline & $\begin{array}{l}\text { Equal variances not } \\
\text { assumed }\end{array}$ & & & -.823 & 66.175 & .414 & -1.22093 & 1.48419 & -4.18406 & 1.74220 \\
\hline \multirow[t]{2}{*}{ LSI_CAR } & $\begin{array}{l}\text { Equal variances } \\
\text { assumed }\end{array}$ & 1.210 & .274 & 1.803 & 99 & .074 & .03089 & .01713 & -.00310 & .06489 \\
\hline & $\begin{array}{l}\text { Equal variances not } \\
\text { assumed }\end{array}$ & & & 2.076 & 72.073 & .041 & .03089 & .01488 & .00122 & .06056 \\
\hline \multirow[t]{2}{*}{ LSI_HM } & $\begin{array}{l}\text { Equal variances } \\
\text { assumed }\end{array}$ & .451 & .504 & -.797 & 87 & .428 & -.16190 & .20313 & -.56565 & .24185 \\
\hline & $\begin{array}{l}\text { Equal variances not } \\
\text { assumed }\end{array}$ & & & -.917 & 51.361 & .364 & -.16190 & .17661 & -.51640 & .19260 \\
\hline \multirow[t]{2}{*}{ LSI_AMT } & $\begin{array}{l}\text { Equal variances } \\
\text { assumed }\end{array}$ & .050 & .824 & .509 & 86 & .612 & .01949 & .03831 & -.05667 & .09564 \\
\hline & $\begin{array}{l}\text { Equal variances not } \\
\text { assumed }\end{array}$ & & & .589 & 36.413 & .560 & .01949 & .03310 & -.04761 & .08658 \\
\hline
\end{tabular}


Table D27. Total variance explained by measures of involved ACL reconstructed limb quadriceps function

\begin{tabular}{|c|c|c|c|c|c|c|c|c|c|}
\hline \multirow[b]{2}{*}{ Component } & \multicolumn{3}{|c|}{ Initial Eigenvalues } & \multicolumn{3}{|c|}{ Extraction Sums of Squared Loadings } & \multicolumn{3}{|c|}{ Rotation Sums of Squared Loadings } \\
\hline & Total & $\%$ of Variance & Cumulative $\%$ & Total & $\%$ of Variance & Cumulative $\%$ & Total & $\%$ of Variance & Cumulative $\%$ \\
\hline 1 & 6.519 & 59.263 & 59.263 & 6.519 & 59.263 & 59.263 & 6.509 & 59.175 & 59.175 \\
\hline 2 & 1.200 & 10.912 & 70.176 & 1.200 & 10.912 & 70.176 & 1.192 & 10.836 & 70.011 \\
\hline 3 & 1.160 & 10.547 & 80.723 & 1.160 & 10.547 & 80.723 & 1.178 & 10.712 & 80.723 \\
\hline 4 & .908 & 8.250 & 88.973 & & & & & & \\
\hline 5 & .636 & 5.781 & 94.754 & & & & & & \\
\hline 6 & .229 & 2.085 & 96.838 & & & & & & \\
\hline 7 & .183 & 1.665 & 98.503 & & & & & & \\
\hline 8 & .085 & .769 & 99.272 & & & & & & \\
\hline 9 & .036 & .327 & 99.599 & & & & & & \\
\hline 10 & .033 & .298 & 99.897 & & & & & & \\
\hline 11 & .011 & .103 & 100.000 & & & & & & \\
\hline
\end{tabular}

Table D28. Component (A) and rotated component (B) matrices for involved ACL reconstructed limb quadriceps function

\begin{tabular}{|c|c|c|c|}
\hline & \multicolumn{3}{|c|}{ Component } \\
\hline & 1 & 2 & 3 \\
\hline $\begin{array}{l}\text { Norm_Power_Ext_180_I } \\
\text { nv }\end{array}$ & .970 & & \\
\hline $\begin{array}{l}\text { Norm_Torque_Ext_180_ } \\
\text { Inv }\end{array}$ & .967 & & \\
\hline Norm_Power_Ext_90_Inv & .967 & & \\
\hline Norm_Work_Ext_90_Inv & .957 & & \\
\hline$\underset{v}{\text { Norm_Torque_Ext_90_In }}$ & .952 & & \\
\hline$\underset{\mathrm{v}}{\text { Norm_Work_Ext_180_In }}$ & .952 & & \\
\hline Norm_MVIC_Torque_Inv & .872 & & \\
\hline HM_Inv & & .674 & -.517 \\
\hline CAR_Inv & .319 & .658 & .369 \\
\hline Fatigue_Inv & & .457 & .441 \\
\hline AMT_MSO_Inv & & & .744 \\
\hline
\end{tabular}

Extraction Method: Principal Component Analysis.

a. 3 components extracted.

\begin{tabular}{llll}
\hline & \multicolumn{3}{c}{ Component } \\
\cline { 2 - 4 } & 1 & 2 & 3 \\
\hline Norm_Power_Ext_180_I & .971 & & \\
nv & & \\
Norm_Torque_Ext_180_ & .967 & & \\
Inv & & & \\
Norm_Power_Ext_90_Inv & .967 & & \\
Norm_Work_Ext_90_Inv & .957 & & \\
Norm_Work_Ext_180_In & .954 & & \\
v & & & \\
Norm_Torque_Ext_90_In & .954 & & \\
v & & & .336 \\
Norm_MVIC_Torque_Inv & .863 & & .813 \\
HM_Inv & & .813 & \\
AMT_MSO_Inv & & -.687 & \\
CAR_Inv & .301 & & .629 \\
Fatigue_Inv & & \\
\hline Extraction Method: Principal Component Analysis. \\
Ron
\end{tabular}

Rotation Method: Varimax with Kaiser Normalization.

a. Rotation converged in 5 iterations.

Table D29. Total variance explained by measures of uninvolved ACL reconstructed limb quadriceps function

\begin{tabular}{|c|c|c|c|c|c|c|c|c|c|}
\hline \multirow[b]{2}{*}{ Component } & \multicolumn{3}{|c|}{ Initial Eigenvalues } & \multicolumn{3}{|c|}{ Extraction Sums of Squared Loadings } & \multicolumn{3}{|c|}{ Rotation Sums of Squared Loadings } \\
\hline & Total & $\%$ of Variance & Cumulative $\%$ & Total & $\%$ of Variance & Cumulative $\%$ & Total & $\%$ of Variance & Cumulative $\%$ \\
\hline 1 & 6.274 & 57.034 & 57.034 & 6.274 & 57.034 & 57.034 & 6.169 & 56.083 & 56.083 \\
\hline 2 & 1.508 & 13.708 & 70.742 & 1.508 & 13.708 & 70.742 & 1.613 & 14.659 & 70.742 \\
\hline 3 & .912 & 8.290 & 79.032 & & & & & & \\
\hline 4 & .855 & 7.775 & 86.807 & & & & & & \\
\hline 5 & .701 & 6.370 & 93.177 & & & & & & \\
\hline 6 & .349 & 3.175 & 96.352 & & & & & & \\
\hline 7 & .183 & 1.667 & 98.019 & & & & & & \\
\hline 8 & .107 & .970 & 98.989 & & & & & & \\
\hline 9 & .064 & .578 & 99.567 & & & & & & \\
\hline 10 & .034 & .308 & 99.875 & & & & & & \\
\hline 11 & .014 & .125 & 100.000 & & & & & & \\
\hline
\end{tabular}


Table D30. Component (A) and rotated component (B) matrices for uninvolved ACL reconstructed limb quadriceps function

\begin{tabular}{|c|c|c|c|c|c|}
\hline & \multicolumn{2}{|c|}{ Component } & & \multicolumn{2}{|c|}{ Component } \\
\hline & 1 & 2 & & 1 & 2 \\
\hline $\begin{array}{l}\text { Norm_Torque_Ext_180_ } \\
\text { Un }\end{array}$ & .956 & & $\begin{array}{l}\text { Norm_Power_Ext_180_U } \\
\mathrm{n}\end{array}$ & .957 & \\
\hline Norm_Power_Ext_90_Un & .955 & & $\begin{array}{l}\text { Norm_Torque_Ext_180_ } \\
\text { Un }\end{array}$ & .956 & \\
\hline $\begin{array}{l}\text { Norm_Power_Ext_180_U } \\
\mathrm{n}\end{array}$ & .952 & & Norm_Work_Ext_90_Un & .952 & \\
\hline Norm_Work_Ext_90_Un & .952 & & Norm_Work_Ext_180_Un & .948 & \\
\hline Norm_Work_Ext_180_Un & .944 & & $\begin{array}{l}\text { Norm_Torque_Ext_90_U } \\
\text { N }\end{array}$ & .947 & \\
\hline $\begin{array}{l}\text { Norm_Torque_Ext_90_U } \\
\text { n }\end{array}$ & .935 & & Norm_Power_Ext_90_Un & .937 & \\
\hline Norm_MVIC_Torque_Un & .830 & & Norm_MVIC_Torque_Un & .814 & \\
\hline AMT_MSO_Un & & .662 & AMT_MSO_Un & & -.696 \\
\hline HM_Un & & -.642 & HM_Un & & .632 \\
\hline CAR_Un & & -.566 & CAR_Un & & .571 \\
\hline Fatigue_Un & -.308 & .530 & Fatigue_Un & & -.569 \\
\hline $\begin{array}{l}\text { Extraction Method: Princi } \\
\text { Analysis. }\end{array}$ & Compon & & $\begin{array}{l}\text { Extraction Method: Princip } \\
\text { Analysis. } \\
\text { Rotation Method: Varima }\end{array}$ & $\begin{array}{l}\text { neon } \\
\text { Kaise }\end{array}$ & \\
\hline
\end{tabular}

a. Rotation converged in 3 iterations.

Table D31. Total variance explained by limb symmetry measures of quadriceps function in ACL reconstructed patients

\begin{tabular}{|c|c|c|c|c|c|c|c|c|c|}
\hline \multirow[b]{2}{*}{ Component } & \multicolumn{3}{|c|}{ Initial Eigenvalues } & \multicolumn{3}{|c|}{ Extraction Sums of Squared Loadings } & \multicolumn{3}{|c|}{ Rotation Sums of Squared Loadings } \\
\hline & Total & $\%$ of Variance & Cumulative $\%$ & Total & $\%$ of Variance & Cumulative $\%$ & Total & $\%$ of Variance & Cumulative $\%$ \\
\hline 1 & 6.529 & 59.358 & 59.358 & 6.529 & 59.358 & 59.358 & 5.945 & 54.045 & 54.045 \\
\hline 2 & 1.148 & 10.441 & 69.799 & 1.148 & 10.441 & 69.799 & 1.638 & 14.891 & 68.936 \\
\hline 3 & 1.006 & 9.142 & 78.941 & 1.006 & 9.142 & 78.941 & 1.101 & 10.005 & 78.941 \\
\hline 4 & .939 & 8.534 & 87.475 & & & & & & \\
\hline 5 & .694 & 6.308 & 93.784 & & & & & & \\
\hline 6 & .287 & 2.612 & 96.396 & & & & & & \\
\hline 7 & .232 & 2.107 & 98.503 & & & & & & \\
\hline 8 & .082 & .749 & 99.252 & & & & & & \\
\hline 9 & .045 & .407 & 99.659 & & & & & & \\
\hline 10 & .025 & .226 & 99.884 & & & & & & \\
\hline 11 & .013 & .116 & 100.000 & & & & & & \\
\hline
\end{tabular}


Table D32. Component (A) and rotated component (B) matrices for limb symmetry measures of quadriceps function in ACL reconstructed patients

\begin{tabular}{lcc}
\hline & \multicolumn{3}{c}{ Component } \\
\cline { 2 - 3 } & 1 & 2 \\
\hline LSI_TotalWork_Ext_90 & .962 & \\
LSI_Avg_Power_Ext_90 & .957 & \\
LSI_Peak_Torque_Ext_1 & .955 & \\
80 & & \\
LSI_Avg_Power_Ext_180 & .948 & \\
LSI_Peak_Torque_Ext_9 & .945 & \\
0 & & \\
LSI_TotalWork_Ext_180 & .942 & \\
LSI_MVIC & .839 & \\
LSI_HM & & \\
LSI_Fatigue_Diff & -.370 & .745 \\
LSI_CAR & .404 & -.622 \\
LSI_AMT & & \\
\hline
\end{tabular}

Extraction Method: Principal Component Analysis. a. 3 components extracted.

\begin{tabular}{llcl}
\hline & \multicolumn{3}{c}{ Component } \\
\cline { 2 - 3 } & 1 & 2 \\
\hline LSI_Avg_Power_Ext_180 & .956 & \\
LSI_TotalWork_Ext_180 & .949 & \\
LSI_Peak_Torque_Ext_1 & .947 & & \\
80 & & & \\
LSI_Avg_Power_Ext_90 & .944 & & \\
LSI_TotalWork_Ext_90 & .934 & & \\
LSI_Peak_Torque_Ext_9 & .928 & & \\
0 & & & \\
LSI_MVIC & .717 & .472 & .948 \\
LSI_Fatigue_Diff & & -.812 & .321 \\
LSI_CAR & & .745 & \\
LSI_AMT & & & \\
LSI_HM & & & \\
\hline
\end{tabular}

\begin{tabular}{l}
\hline Extraction Method: Principal Component Analysis. \\
Rotation Method: Varimax with Kaiser Normalization.
\end{tabular}

321 Rotation Method: Varimax with Kaiser 
Figure D11. Receiver operator characteristic (ROC) curves using measures of involved limb quadriceps function to determine group membership

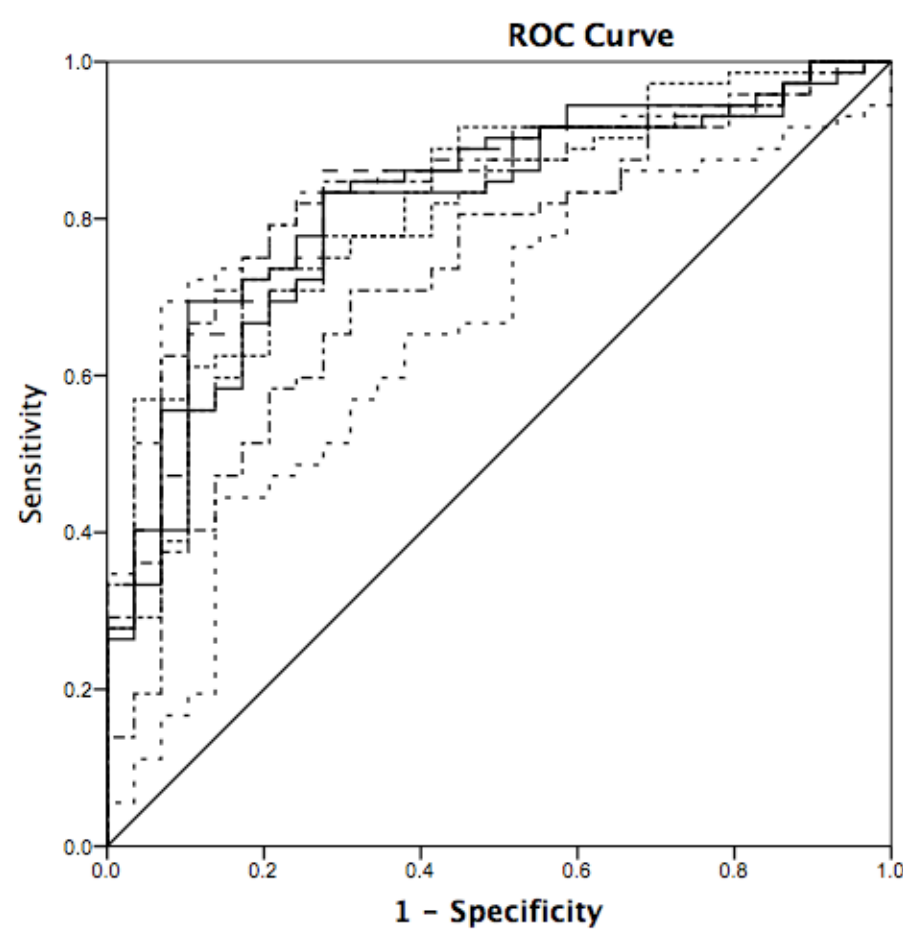

Source of the Curve

- Norm_Torque_Ext_90

- Norm

- Norm_-Work_Ext_-90_-

- Norm_Work_Ext_180_

- - Norm_Power_Ext_90_-

- Norm_Power_Ext_180

-.- Norm_MVIC_Torque_Ir

- Fatigue_Inv

- - CAR_Inv

— Reference Line

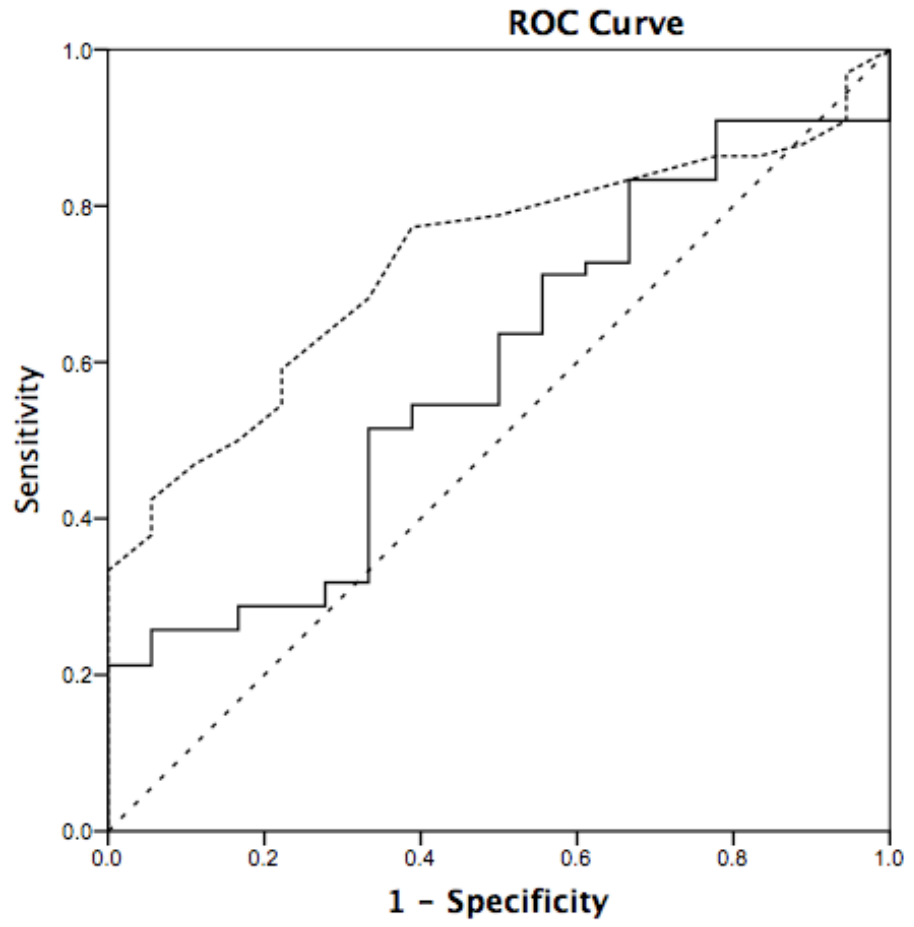

Source of the Curve

.... AMT_MSO_Inv

- Reference Line 
Table D33. Area under the receiver operator characteristic (ROC) curve for measures of involved limb quadriceps function to determine group membership

\begin{tabular}{|c|c|c|c|c|c|}
\hline \multirow[b]{2}{*}{ Test Result Variable(s) } & \multirow[b]{2}{*}{ Area } & \multirow[b]{2}{*}{ Std. Error ${ }^{\mathrm{a}}$} & \multirow{2}{*}{$\begin{array}{l}\text { Asymptotic } \\
\text { Sig. }\end{array}$} & \multicolumn{2}{|c|}{$\begin{array}{c}\text { Asymptotic } 95 \% \text { Confidence } \\
\text { Interval }\end{array}$} \\
\hline & & & & Lower Bound & Upper Bound \\
\hline Norm_Torque_Ext_90_In & .828 & .043 & .000 & .744 & .912 \\
\hline $\begin{array}{l}\text { Norm_Torque_Ext_180_ } \\
\text { Inv }\end{array}$ & .802 & .047 & .000 & .709 & .894 \\
\hline Norm_Work_Ext_90_Inv & .848 & .039 & .000 & .771 & .925 \\
\hline$\underset{\mathrm{v}}{\text { Norm_Work_Ext_180_In }}$ & .829 & .043 & .000 & .744 & .913 \\
\hline Norm_Power_Ext_90_Inv & .833 & .042 & .000 & .750 & .917 \\
\hline $\begin{array}{l}\text { Norm_Power_Ext_180_I } \\
\text { nv }\end{array}$ & .807 & .046 & .000 & .716 & .898 \\
\hline Norm_MVIC_Torque_Inv & .821 & .042 & .000 & .739 & .903 \\
\hline Fatigue_Inv & .649 & .060 & .019 & .533 & .766 \\
\hline CAR_Inv & .731 & .054 & .000 & .626 & .836 \\
\hline
\end{tabular}

a. Under the nonparametric assumption

b. Null hypothesis: true area $=0.5$

\begin{tabular}{|c|c|c|c|c|c|}
\hline \multirow[b]{2}{*}{ Test Result Variable(s) } & \multirow[b]{2}{*}{ Area } & \multirow[b]{2}{*}{ Std. Error ${ }^{\mathrm{a}}$} & \multirow{2}{*}{$\begin{array}{l}\text { Asymptotic } \\
\text { Sig. }\end{array}$} & \multicolumn{2}{|c|}{$\begin{array}{l}\text { Asymptotic 95\% Confidence } \\
\text { Interval }\end{array}$} \\
\hline & & & & Lower Bound & Upper Bound \\
\hline HM_Inv & .589 & .072 & .248 & .448 & .731 \\
\hline AMT_MSO_Inv & .729 & .057 & .003 & .617 & .840 \\
\hline
\end{tabular}

The test result variable(s): AMT_MSO_Inv has at least one tie between the positive actual state group and the negative actual state group. Statistics may be biased.

a. Under the nonparametric assumption

b. Null hypothesis: true area $=0.5$ 
Figure D12. Receiver operator characteristic (ROC) curves using measures of uninvolved limb quadriceps function to determine group membership

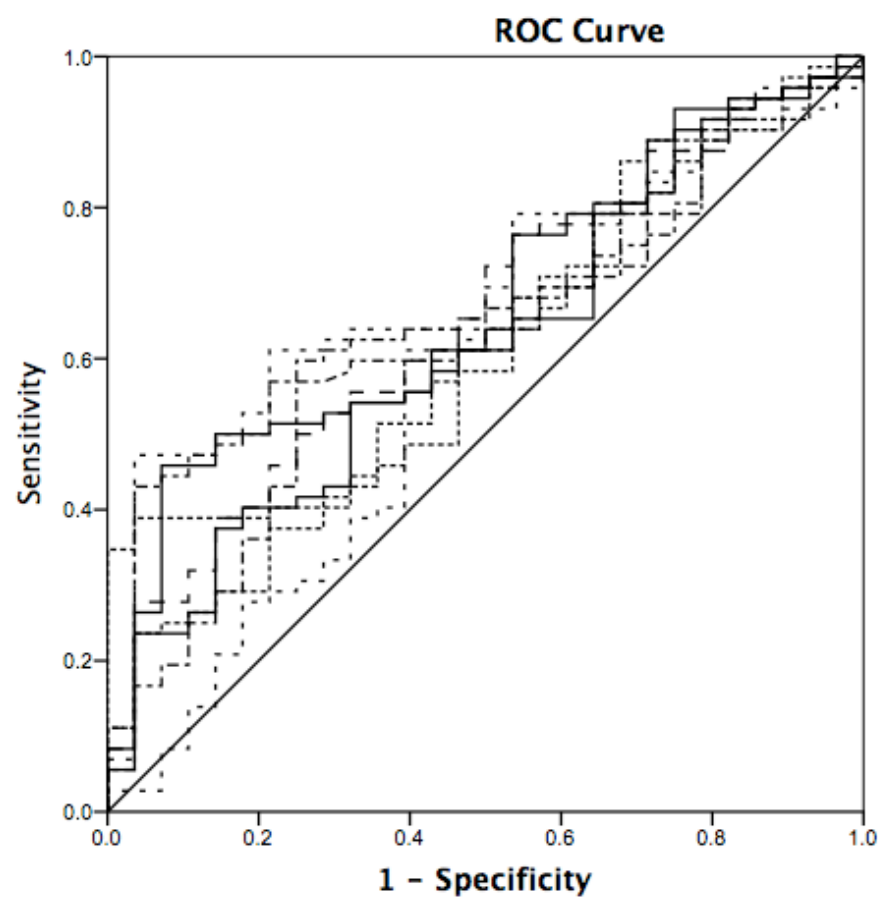

Source of the Curve - Norm_Torque_Ext_90 - Norm Work Ext 90

- Norm Work

- Norm Power

- Norm Power.

- Norm_Power_Ext_180

-.- Norm_MVIC_Torque_U

- Fatigue_Un

- Reference Line

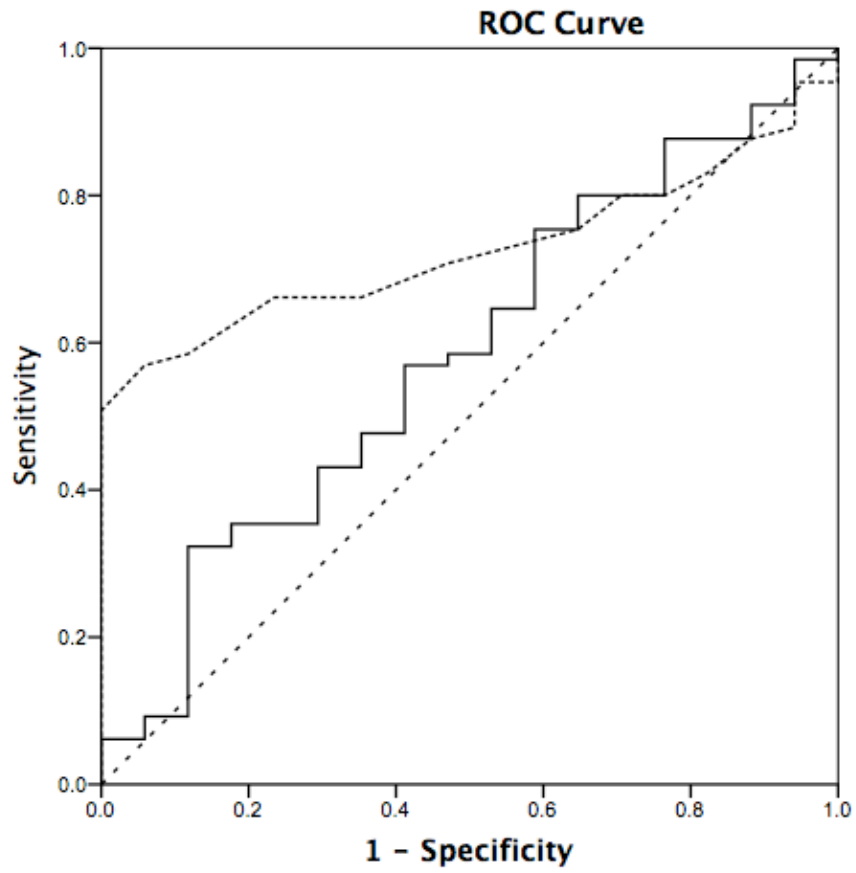

Source of the Curve

- HM_Un .... AMT_MSO Un -. Reference Line 
Table D34. Area under the receiver operator characteristic (ROC) curve for measures of uninvolved limb quadriceps function to determine group membership

\begin{tabular}{|c|c|c|c|c|c|}
\hline \multirow[b]{2}{*}{ Test Result Variable(s) } & \multirow[b]{2}{*}{ Area } & \multirow[b]{2}{*}{ Std. Error ${ }^{\mathrm{a}}$} & \multirow{2}{*}{$\begin{array}{c}\text { Asymptotic } \\
\text { Sig. }\end{array}$} & \multicolumn{2}{|c|}{$\begin{array}{c}\text { Asymptotic } 95 \% \text { Confidence } \\
\text { Interval }\end{array}$} \\
\hline & & & & Lower Bound & Upper Bound \\
\hline $\begin{array}{l}\text { Norm_Torque_Ext_90_U } \\
\text { n }\end{array}$ & .654 & .057 & .017 & .542 & .766 \\
\hline $\begin{array}{l}\text { Norm_Torque_Ext_180_ } \\
\text { Un }\end{array}$ & .593 & .061 & .149 & .473 & .713 \\
\hline Norm_Work_Ext_90_Un & .685 & .054 & .004 & .578 & .791 \\
\hline Norm_Work_Ext_180_Un & .651 & .058 & .020 & .537 & .765 \\
\hline Norm_Power_Ext_90_Un & .661 & .056 & .012 & .552 & .770 \\
\hline $\begin{array}{l}\text { Norm_Power_Ext_180_U } \\
\mathrm{n}\end{array}$ & .633 & .060 & .039 & .515 & .752 \\
\hline Norm_MVIC_Torque_Un & .636 & .057 & .035 & .524 & .748 \\
\hline Fatigue_Un & .581 & .067 & .211 & .450 & .711 \\
\hline CAR_Un & .629 & .060 & .045 & .512 & .747 \\
\hline
\end{tabular}

The test result variable(s): Norm_Power_Ext_90_Un has at least one tie between the positive actual state group and the negative actual state group. Statistics may be biased.

a. Under the nonparametric assumption

b. Null hypothesis: true area $=0.5$

\begin{tabular}{lcccrr}
\hline & & & \multicolumn{2}{c}{$\begin{array}{c}\text { Asymptotic 95\% Confidence } \\
\text { Interval }\end{array}$} \\
\cline { 5 - 6 } Test Result Variable(s) & Area & Std. Error & $\begin{array}{c}\text { Asymptotic } \\
\text { Sig. }\end{array}$ & Lower Bound & Upper Bound \\
\hline HM_Un & .583 & .077 & .295 & .432 & .733 \\
AMT_MSO_Un & .725 & .053 & .004 & .621 & .830 \\
\hline
\end{tabular}

The test result variable(s): AMT_MSO_Un has at least one tie between the positive actual state group and the negative actual state group. Statistics may be biased.

a. Under the nonparametric assumption

b. Null hypothesis: true area $=0.5$ 
Figure D13. Receiver operator characteristic (ROC) curves using limb symmetry measures of quadriceps function to determine group membership
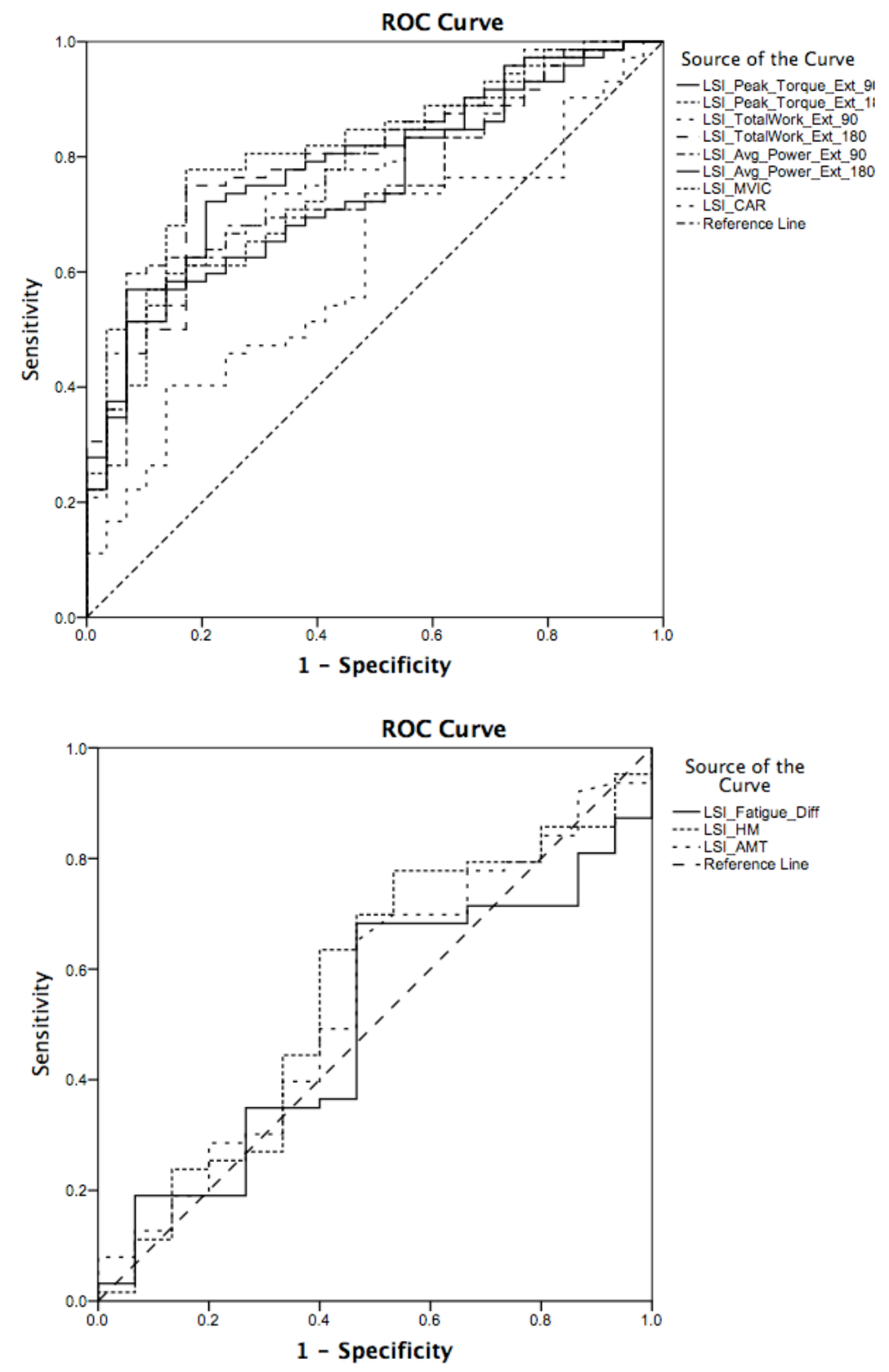
Table D35. Area under the receiver operator characteristic (ROC) curve for limb symmetry measures of quadriceps function to determine group membership

\begin{tabular}{|c|c|c|c|c|c|}
\hline \multirow[b]{2}{*}{ Test Result Variable(s) } & \multirow[b]{2}{*}{ Area } & \multirow[b]{2}{*}{ Std. Error ${ }^{\mathrm{a}}$} & \multirow{2}{*}{$\begin{array}{l}\text { Asymptotic } \\
\text { Sig. }\end{array}$} & \multicolumn{2}{|c|}{$\begin{array}{l}\text { Asymptotic 95\% Confidence } \\
\text { Interval }\end{array}$} \\
\hline & & & & Lower Bound & Upper Bound \\
\hline LSI_Peak_Torque_Ext_9 & .752 & .050 & .000 & .655 & .850 \\
\hline $\begin{array}{l}\text { LSI_Peak_Torque_Ext_1 } \\
80\end{array}$ & .808 & .046 & .000 & .718 & .898 \\
\hline LSI_TotalWork_Ext_90 & .773 & .048 & .000 & .680 & .867 \\
\hline LSI_TotalWork_Ext_180 & .788 & .047 & .000 & .697 & .880 \\
\hline LSI_Avg_Power_Ext_90 & .750 & .050 & .000 & .652 & .848 \\
\hline LSI_Avg_Power_Ext_180 & .780 & .047 & .000 & .688 & .872 \\
\hline LSI_MVIC & .776 & .047 & .000 & .683 & .869 \\
\hline LSI_CAR & .610 & .059 & .084 & .495 & .726 \\
\hline
\end{tabular}

a. Under the nonparametric assumption

b. Null hypothesis: true area $=0.5$

\begin{tabular}{lcccrr}
\hline & & & \multicolumn{2}{c}{$\begin{array}{c}\text { Asymptotic 95\% Confidence } \\
\text { Interval }\end{array}$} \\
\cline { 5 - 6 } Test Result Variable(s) & Area & Std. Error & $\begin{array}{c}\text { Asymptotic } \\
\text { Sig. }\end{array}$ & Lower Bound & Upper Bound \\
\hline LSI_Fatigue_Diff & .503 & .080 & .975 & .346 & .659 \\
LSI_HM & .565 & .086 & .436 & .397 & .733 \\
LSI_AMT & .548 & .083 & .564 & .385 & .711 \\
\hline
\end{tabular}

The test result variable(s): LSI_AMT has at least one tie between the positive actual state group and the negative actual state group. Statistics may be biased.

a. Under the nonparametric assumption

b. Null hypothesis: true area $=0.5$

Table D36. Binary logistic regression results to predict group membership using measures of involved limb quadriceps function

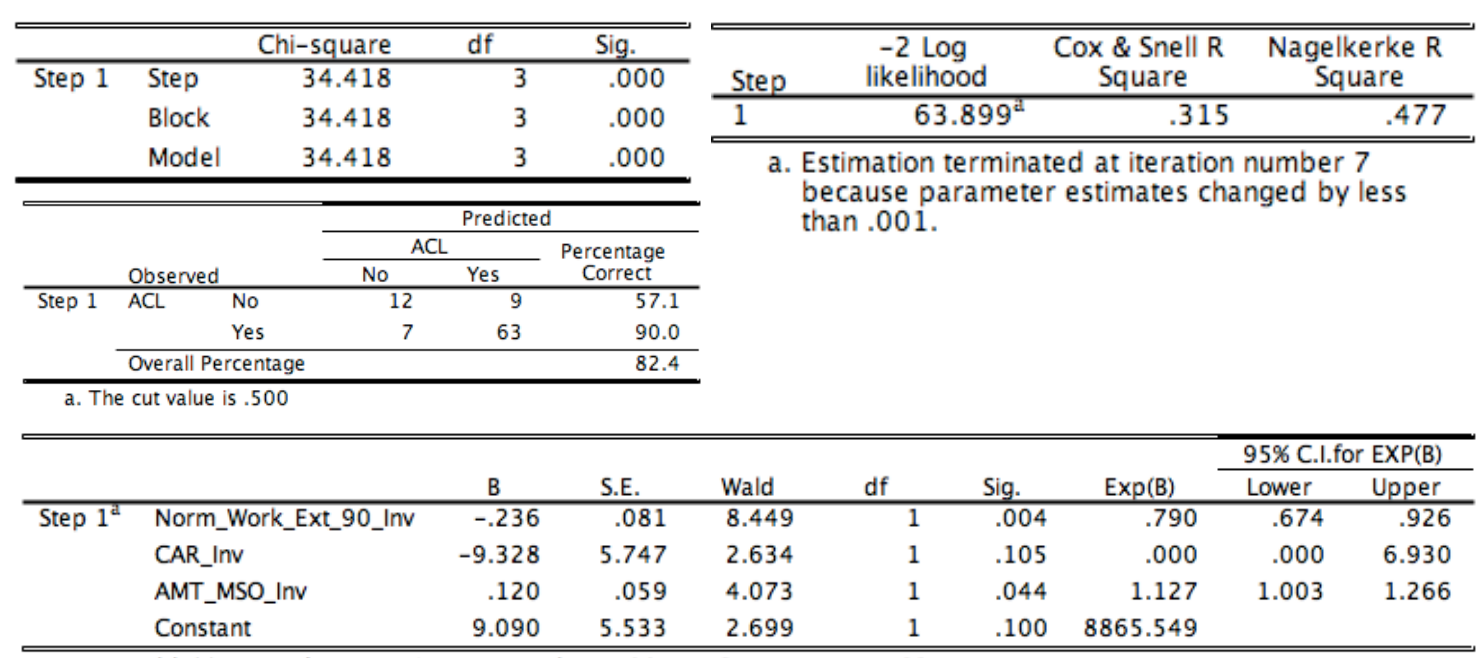

a. Variable(s) entered on step 1: Norm_Work_Ext_90_Inv, CAR_Inv, AMT_MSO_Inv. 
Table D37. Binary logistic regression results to predict group membership using measures of uninvolved limb quadriceps function

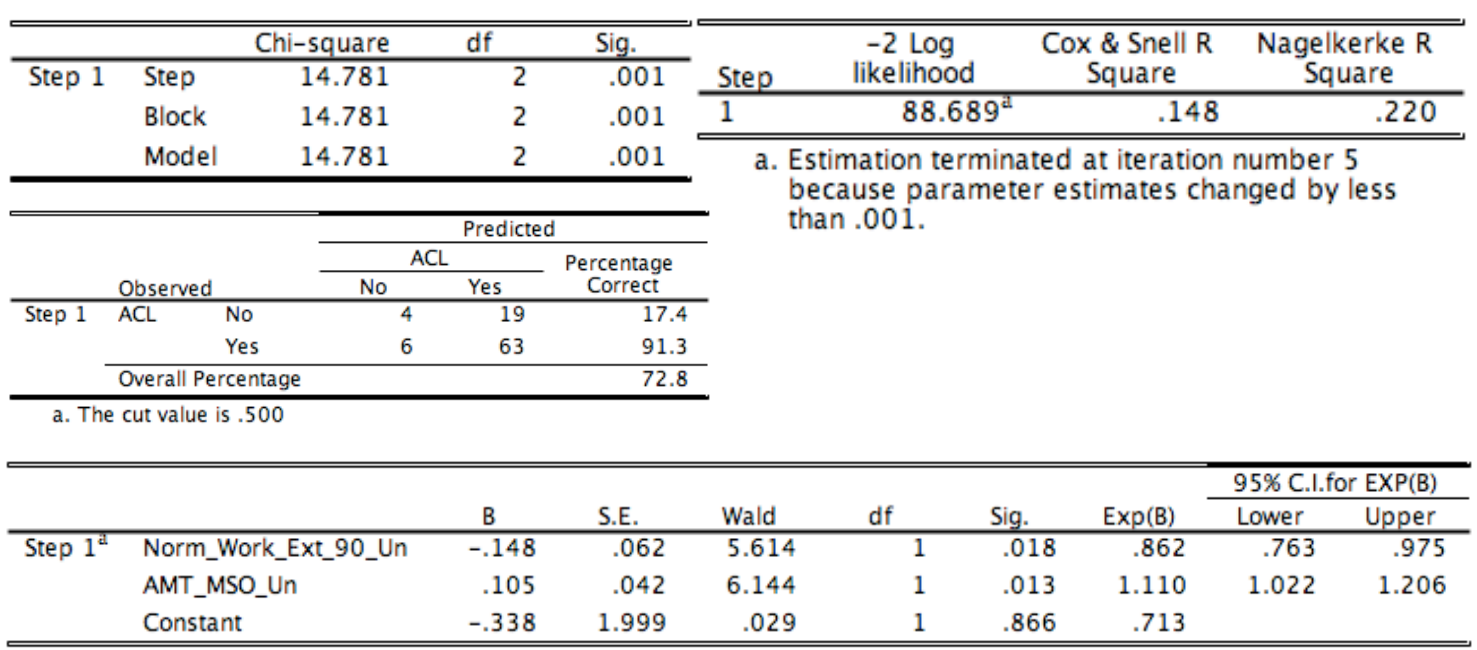

a. Variable(s) entered on step 1: Norm_Work_Ext_90_Un, AMT_MSO_Un.

Table D38. Binary logistic regression results to predict group membership using limb symmetry measures of quadriceps function

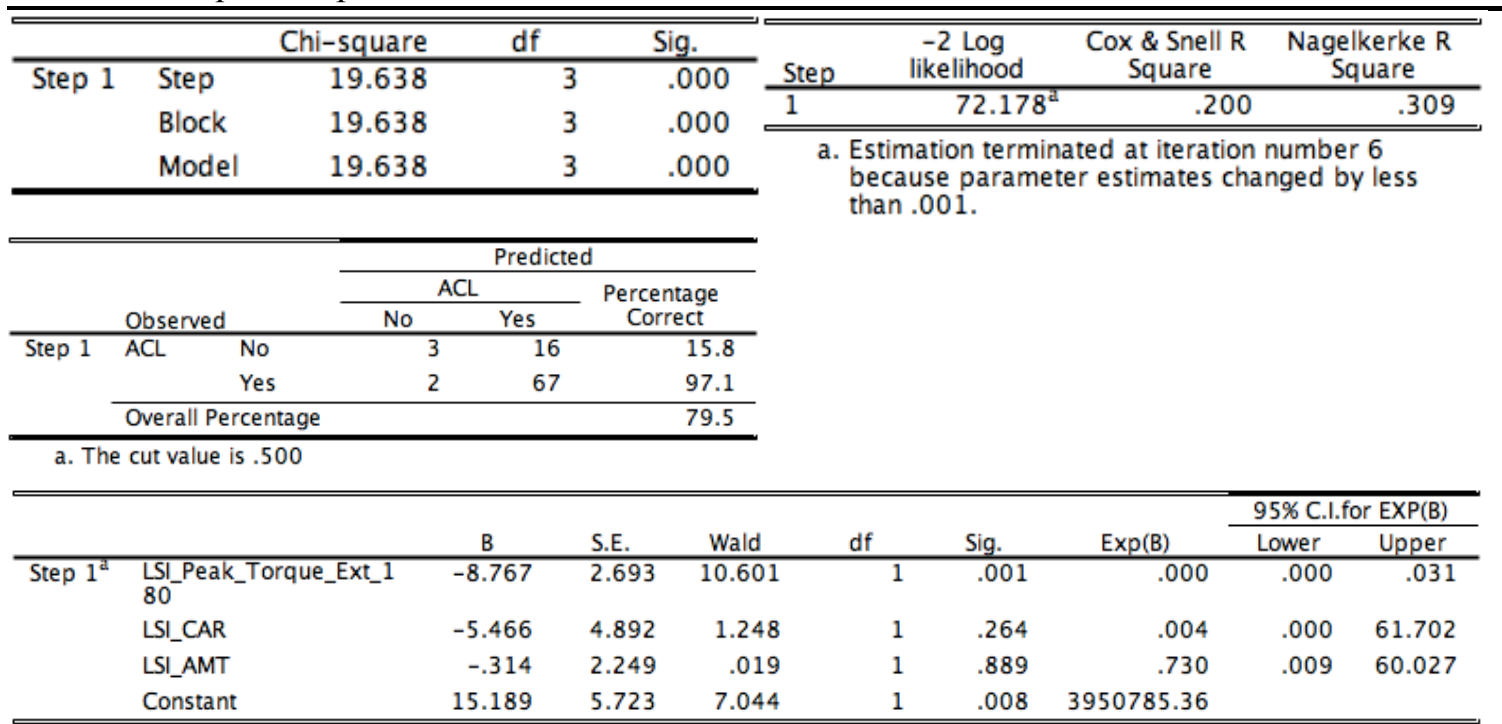

a. Variable(s) entered on step 1: LSI_Peak_Torque_Ext_180, LSI_CAR, LSI_AMT. 


\section{APPENDIX E}

\section{Back Matter}

\section{Recommendations for future research}

1. Examine the natural history of post-traumatic lower extremity neuromuscular function following ACL reconstruction using a prospective longitudinal study design.

2. Does quadriceps neuromuscular function differ between patients with a clinical diagnosis (WOMAC score) of knee osteoarthritis compared to those with radiographic evidence?

3. Do early mal-adaptive patterns of quadriceps neuromuscular function influence long-term patient outcomes?

4. Which treatment strategy, or strategies, is most effective to treat specific neuromuscular impairments (i.e. muscle weakness, central activation failure, reflex inhibition, decreased corticospinal drive)?

5. Does early treatment of measured neuromuscular impairments influence patient outcomes? Can a minimally clinically important difference be established for common estimates of neuromuscular function (i.e. knee extensor torque, central activation, spinal reflexive excitability, corticospinal excitability)?

6. Does a threshold of time from surgery exist in which patients become less responsive to known treatment strategies for neuromuscular impairments?

7. What are the cutoff values for common estimates of neuromuscular function to discriminate between healthy individuals and ACL reconstructed patients with and without knee osteoarthritis? 


\section{BIBLIOGRAPHY}

1. Hart JM, Pietrosimone B, Hertel J, Ingersoll CD. Quadriceps activation following knee injuries: a systematic review. J Athl Train. Jan-Feb 2010;45(1):87-97.

2. Lohmander LS, Ostenberg A, Englund M, Roos H. High prevalence of knee osteoarthritis, pain, and functional limitations in female soccer players twelve years after anterior cruciate ligament injury. Arthritis Rheum. Oct 2004;50(10):3145-3152.

3. Ericsson YB, Roos EM, Dahlberg L. Muscle strength, functional performance, and selfreported outcomes four years after arthroscopic partial meniscectomy in middle-aged patients. Arthritis Rheum. Dec 15 2006;55(6):946-952.

4. Fitzgerald GK, Piva SR, Irrgang JJ, Bouzubar F, Starz TW. Quadriceps activation failure as a moderator of the relationship between quadriceps strength and physical function in individuals with knee osteoarthritis. Arthritis Rheum. Feb 15 2004;51(1):40-48.

5. Yoshida Y, Mizner RL, Ramsey DK, Snyder-Mackler L. Examining outcomes from total knee arthroplasty and the relationship between quadriceps strength and knee function over time. Clin Biomech. Mar 2008;23(3):320-328.

6. Paterno MV, Schmitt LC, Ford KR, et al. Biomechanical measures during landing and postural stability predict second anterior cruciate ligament injury after anterior cruciate ligament reconstruction and return to sport. Am J Sports Med. Oct 2010;38(10):19681978.

7. Brandt KD, Dieppe P, Radin EL. Etiopathogenesis of osteoarthritis. Rheum Dis Clin North Am. Aug 2008;34(3):531-559.

8. Mikesky AE, Mazzuca SA, Brandt KD, Perkins SM, Damush T, Lane KA. Effects of strength training on the incidence and progression of knee osteoarthritis. Arthritis Rheum. Oct 15 2006;55(5):690-699.

9. Palmieri-Smith RM, Thomas AC. A neuromuscular mechanism of posttraumatic osteoarthritis associated with ACL injury. Exerc Sport Sci Rev. Jul 2009;37(3):147-153.

10. Slemenda C, Heilman DK, Brandt KD, et al. Reduced quadriceps strength relative to body weight: a risk factor for knee osteoarthritis in women? Arthritis Rheum. Nov 1998;41(11):1951-1959.

11. Roos EM, Herzog W, Block JA, Bennell KL. Muscle weakness, afferent sensory dysfunction and exercise in knee osteoarthritis. Nat Rev Rheumatol. Jan 2011;7(1):57-63.

12. Hart JM, Turman KA, Diduch DR, Hart JA, Miller MD. Quadriceps muscle activation and radiographic osteoarthritis following ACL revision. Knee Surg Sports Traumatol Arthrosc. Apr 2011;19(4):634-640.

13. Louboutin H, Debarge R, Richou J, et al. Osteoarthritis in patients with anterior cruciate ligament rupture: a review of risk factors.Knee. Aug 2009;16(4):239-244.

14. Oiestad BE, Holm I, Engebretsen L, Risberg MA. The association between radiographic knee osteoarthritis and knee symptoms, function and quality of life 10-15 years after anterior cruciate ligament reconstruction. Br J Sports Med. Jun 2011;45(7):583-588.

15. Sanes JN, Donoghue JP. Plasticity and primary motor cortex. Annu Rev Neurosci. 2000;23:393-415. 
16. Kapreli E, Athanasopoulos S, Gliatis J, et al. Anterior cruciate ligament deficiency causes brain plasticity: a functional MRI study. Am J Sports Med. Dec 2009;37(12):2419-2426.

17. Baumeister J, Reinecke K, Weiss M. Changed cortical activity after anterior cruciate ligament reconstruction in a joint position paradigm: an EEG study. Scand J Med Sci Sports. Aug 2008;18(4):473-484.

18. Lepley AS, Ericksen HM, Sohn DH, Pietrosimone BG. Contributions of neural excitability and voluntary activation to quadriceps muscle strength following anterior cruciate ligament reconstruction. Knee. Feb 162014.

19. Rice DA, McNair PJ. Quadriceps arthrogenic muscle inhibition: neural mechanisms and treatment perspectives. Semin Arthritis Rheum. Dec 2010;40(3):250-266.

20. Torry MR, Decker MJ, Viola RW, O'Connor DD, Steadman JR. Intra-articular knee joint effusion induces quadriceps avoidance gait patterns. Clin Biomech. Mar 2000;15(3):147159.

21. Klykken LW, Pietrosimone BG, Kim KM, Ingersoll CD, Hertel J. Motor-neuron pool excitability of the lower leg muscles after acute lateral ankle sprain. J Athl Train. MayJun 2011;46(3):263-269.

22. Anand S, Hotson J. Transcranial magnetic stimulation: neurophysiological applications and safety. Brain Cogn. Dec 2002;50(3):366-386.

23. Riemann BL, Lephart SM. The sensorimotor system, part I: the physiologic basis of functional joint stability. J Athl Train. Jan 2002;37(1):71-79.

24. Heroux ME, Tremblay F. Corticomotor excitability associated with unilateral knee dysfunction secondary to anterior cruciate ligament injury. Knee Surg Sports Traumatol Arthrosc. Sep 2006;14(9):823-833.

25. Groppa S, Oliviero A, Eisen A, et al. A practical guide to diagnostic transcranial magnetic stimulation: report of an IFCN committee. Clin Neurophysiol. May 2012;123(5):858-882.

26. Hopkins JT, Ingersoll CD. Arthrogenic muscle inhibition: a limiting factor in joint rehabilitation. J Sport Rehabil. 2000;9:135-159.

27. Bavelier D, Neville HJ. Cross-modal plasticity: where and how? Nat Rev Neurosci. Jun 2002;3(6):443-452.

28. Todd G, Butler JE, Gandevia SC, Taylor JL. Decreased input to the motor cortex increases motor cortical excitability. Clin Neurophysiol. Nov 2006;117(11):2496-2503.

29. Schutter DJ, van Honk J. A standardized motor threshold estimation procedure for transcranial magnetic stimulation research. $J E C T$. Sep 2006;22(3):176-178.

30. Palmieri RM, Ingersoll CD, Hoffman MA. The hoffmann reflex: methodologic considerations and applications for use in sports medicine and athletic training research. $J$ Athl Train. Jul 2004;39(3):268-277.

31. Zehr EP. Considerations for use of the Hoffmann reflex in exercise studies. Eur J Appl Physiol. Apr 2002;86(6):455-468.

32. CS S, ed The Integrative Action of the Nervous System. New York, NY: C Scribner's Sons; 1906.

33. Kent-Braun JA, Le Blanc R. Quantitation of central activation failure during maximal voluntary contractions in humans. Muscle Nerve. Jul 1996;19(7):861-869.

34. Baumeister J, von Detten S, van Niekerk SM, Schubert M, Ageberg E, Louw QA. Brain activity in predictive sensorimotor control for landings: an EEG pilot study. Int J Sports Med. Dec 2013;34(12):1106-1111.

35. Lephart SM RB, Fu FH. Introduction to the sensorimotor system. In: Lephart SM FF, ed. Proprioception and Neuromuscular Control in Joint Stability. Champaign, IL: Human Kinetics; 2000:37-51.

36. AC G, ed Textbook of Medical Physiology. 8th ed. Philadephia, PA: WB Saunders; 1992. 
37. Rutherford OM, Jones DA, Newham DJ. Clinical and experimental application of the percutaneous twitch superimposition technique for the study of human muscle activation. J Neurol Neurosurg Psychiatry. Nov 1986;49(11):1288-1291.

38. Stackhouse SK, Dean JC, Lee SC, Binder-MacLeod SA. Measurement of central activation failure of the quadriceps femoris in healthy adults. Muscle Nerve. Nov 2000;23(11):1706-1712.

39. Snyder-Mackler L, De Luca PF, Williams PR, Eastlack ME, Bartolozzi AR, 3rd. Reflex inhibition of the quadriceps femoris muscle after injury or reconstruction of the anterior cruciate ligament. J Bone Joint Surg Am. Apr 1994;76(4):555-560.

40. Hootman JM, Dick R, Agel J. Epidemiology of collegiate injuries for 15 sports: summary and recommendations for injury prevention initiatives. $J$ Athl Train. Apr-Jun 2007;42(2):311-319.

41. Beynnon BD, Johnson RJ, Abate JA, Fleming BC, Nichols CE. Treatment of anterior cruciate ligament injuries, part I. Am J Sports Med. Oct 2005;33(10):1579-1602.

42. Marx RG, Jones EC, Angel M, Wickiewicz TL, Warren RF. Beliefs and attitudes of members of the American Academy of Orthopaedic Surgeons regarding the treatment of anterior cruciate ligament injury. Arthroscopy. Sep 2003;19(7):762-770.

43. Mall NA, Chalmers PN, Moric M, et al. Incidence and trends of anterior cruciate ligament reconstruction in the United States. Am J Sports Med. Oct 2014;42(10):23632370.

44. Lyman S, Koulouvaris P, Sherman S, Do H, Mandl LA, Marx RG. Epidemiology of anterior cruciate ligament reconstruction: trends, readmissions, and subsequent knee surgery. J Bone Joint Surg Am. Oct 2009;91(10):2321-2328.

45. Majewski M, Susanne H, Klaus S. Epidemiology of athletic knee injuries: A 10-year study. Knee. Jun 2006;13(3):184-188.

46. Gianotti SM, Marshall SW, Hume PA, Bunt L. Incidence of anterior cruciate ligament injury and other knee ligament injuries: a national population-based study. $J$ Sci Med Sport. Nov 2009;12(6):622-627.

47. Prodromos CC, Han Y, Rogowski J, Joyce B, Shi K. A meta-analysis of the incidence of anterior cruciate ligament tears as a function of gender, sport, and a knee injury-reduction regimen. Arthroscopy. Dec 2007;23(12):1320-1325 e1326.

48. Barber-Westin SD, Noyes FR, Smith ST, Campbell TM. Reducing the risk of noncontact anterior cruciate ligament injuries in the female athlete. Phys Sportsmed. Oct 2009;37(3):49-61.

49. Griffin LY, Agel J, Albohm MJ, et al. Noncontact anterior cruciate ligament injuries: risk factors and prevention strategies. J Am Acad Orthop Surg. May-Jun 2000;8(3):141-150.

50. Hewett TE. Neuromuscular and hormonal factors associated with knee injuries in female athletes. Strategies for intervention. Sports Med. May 2000;29(5):313-327.

51. Hewett TE, Myer GD, Ford KR. Reducing knee and anterior cruciate ligament injuries among female athletes: a systematic review of neuromuscular training interventions. $J$ Knee Surg. Jan 2005;18(1):82-88.

52. Mohtadi NG, Chan DS, Dainty KN, Whelan DB. Patellar tendon versus hamstring tendon autograft for anterior cruciate ligament rupture in adults. Cochrane Database Syst Rev. 2011(9):CD005960.

53. Leys T, Salmon L, Waller A, Linklater J, Pinczewski L. Clinical results and risk factors for reinjury 15 years after anterior cruciate ligament reconstruction: a prospective study of hamstring and patellar tendon grafts. Am J Sports Med. Mar 2012;40(3):595-605.

54. Bjornsson H, Andernord D, Desai N, et al. No Difference in Revision Rates Between Single- and Double-Bundle Anterior Cruciate Ligament Reconstruction: A Comparative Study of 16,791 Patients From the Swedish National Knee Ligament Register.

Arthroscopy. Jan 212015. 
55. Shelbourne KD, Gray $T$, Haro M. Incidence of subsequent injury to either knee within 5 years after anterior cruciate ligament reconstruction with patellar tendon autograft. $A m J$ Sports Med. Feb 2009;37(2):246-251.

56. Brophy RH, Schmitz L, Wright RW, et al. Return to play and future ACL injury risk after ACL reconstruction in soccer athletes from the Multicenter Orthopaedic Outcomes Network (MOON) group. Am J Sports Med. Nov 2012;40(11):2517-2522.

57. Paterno MV, Rauh MJ, Schmitt LC, Ford KR, Hewett TE. Incidence of contralateral and ipsilateral anterior cruciate ligament (ACL) injury after primary ACL reconstruction and return to sport. Clin J Sport Med. Mar 2012;22(2):116-121.

58. Paterno MV, Rauh MJ, Schmitt LC, Ford KR, Hewett TE. Incidence of Second ACL Injuries 2 Years After Primary ACL Reconstruction and Return to Sport. Am J Sports Med. Apr 21 2014;42(7):1567-1573.

59. Tiamklang T, Sumanont S, Foocharoen T, Laopaiboon M. Double-bundle versus singlebundle reconstruction for anterior cruciate ligament rupture in adults. Cochrane Database Syst Rev. 2012;11:CD008413.

60. Faltstrom A, Hagglund M, Magnusson H, Forssblad M, Kvist J. Predictors for additional anterior cruciate ligament reconstruction: data from the Swedish national ACL register. Knee Surg Sports Traumatol Arthrosc. Nov 12014.

61. Laboute E, Savalli L, Puig P, et al. Analysis of return to competition and repeat rupture for 298 anterior cruciate ligament reconstructions with patellar or hamstring tendon autograft in sportspeople. Ann Phys Rehabil Med. Dec 2010;53(10):598-614.

62. Trojian $\mathrm{TH}$, Collins $\mathrm{S}$. The anterior cruciate ligament tear rate varies by race in professional Women's basketball. Am J Sports Med. Jun 2006;34(6):895-898.

63. Shelbourne KD, Gray $\mathrm{T}$, Benner RW. Intercondylar notch width measurement differences between African American and white men and women with intact anterior cruciate ligament knees. Am J Sports Med. Aug 2007;35(8):1304-1307.

64. Myer GD, Martin L, Jr., Ford KR, et al. No association of time from surgery with functional deficits in athletes after anterior cruciate ligament reconstruction: evidence for objective return-to-sport criteria. Am J Sports Med. Oct 2012;40(10):2256-2263.

65. Magnussen RA, Mansour AA, Carey JL, Spindler KP. Meniscus status at anterior cruciate ligament reconstruction associated with radiographic signs of osteoarthritis at 5to 10-year follow-up: a systematic review. J Knee Surg. Oct 2009;22(4):347-357.

66. Segal NA, Torner JC, Felson D, et al. Effect of thigh strength on incident radiographic and symptomatic knee osteoarthritis in a longitudinal cohort. Arthritis Rheum. Sep 15 2009;61(9):1210-1217.

67. Pietrosimone BG, Lepley AS, Ericksen HM, Gribble PA, Levine J. Quadriceps strength and corticospinal excitability as predictors of disability after anterior cruciate ligament reconstruction. J Sport Rehabil. Feb 2013;22(1):1-6.

68. Lepley AS, Ericksen HM, Sohn DH, Pietrosimone BG. Contributions of neural excitability and voluntary activation to quadriceps muscle strength following anterior cruciate ligament reconstruction. Knee. Jun 2014;21(3):736-742.

69. Stokes M, Young A. The contribution of reflex inhibition to arthrogenous muscle weakness. Clin Sci (Lond). Jul 1984;67(1):7-14.

70. Hopkins JT, Ingersoll CD, Krause BA, Edwards JE, Cordova ML. Effect of knee joint effusion on quadriceps and soleus motoneuron pool excitability. Med Sci Sports Exerc. Jan 2001;33(1):123-126.

71. Palmieri-Smith RM, Villwock M, Downie B, Hecht G, Zernicke R. Pain and effusion and quadriceps activation and strength. $J$ Athl Train. Mar-Apr 2013;48(2):186-191.

72. Luc B, Gribble PA, Pietrosimone BG. Osteoarthritis prevalence following anterior cruciate ligament reconstruction: a systematic review and numbers-needed-to-treat analysis. J Athl Train. Nov-Dec 2014;49(6):806-819. 
73. Neuman P, Kostogiannis I, Friden T, Roos H, Dahlberg LE, Englund M. Patellofemoral osteoarthritis 15 years after anterior cruciate ligament injury--a prospective cohort study. Osteoarthritis Cartilage. Mar 2009;17(3):284-290.

74. Ardern CL, Taylor NF, Feller JA, Webster KE. Fifty-five per cent return to competitive sport following anterior cruciate ligament reconstruction surgery: an updated systematic review and meta-analysis including aspects of physical functioning and contextual factors. Br J Sports Med. Nov 2014;48(21):1543-1552.

75. Ardern CL, Osterberg A, Tagesson S, Gauffin H, Webster KE, Kvist J. The impact of psychological readiness to return to sport and recreational activities after anterior cruciate ligament reconstruction. Br J Sports Med. Dec 2014;48(22):1613-1619.

76. Ardern CL, Taylor NF, Feller JA, Whitehead TS, Webster KE. Psychological responses matter in returning to preinjury level of sport after anterior cruciate ligament reconstruction surgery. Am J Sports Med. Jul 2013;41(7):1549-1558.

77. Ardern CL, Taylor NF, Feller JA, Webster KE. Return-to-sport outcomes at 2 to 7 years after anterior cruciate ligament reconstruction surgery. Am J Sports Med. Jan 2012;40(1):41-48.

78. Schmale GA, Kweon C, Larson RV, Bompadre V. High satisfaction yet decreased activity 4 years after transphyseal ACL reconstruction. Clin Orthop Relat Res. Jul 2014;472(7):2168-2174.

79. Geborek P, Mansson B, Wollheim FA, Moritz U. Intraarticular corticosteroid injection into rheumatoid arthritis knees improves extensor muscles strength. Rheumatol Int. 1990;9(6):265-270.

80. Thomas AC, Villwock M, Wojtys EM, Palmieri-Smith RM. Lower extremity muscle strength after anterior cruciate ligament injury and reconstruction. $J$ Athl Train. Sep-Oct 2013;48(5):610-620.

81. Macleod TD, Snyder-Mackler L, Buchanan TS. Differences in neuromuscular control and quadriceps morphology between potential copers and noncopers following anterior cruciate ligament injury. J Orthop Sports Phys Ther. Feb 2014;44(2):76-84.

82. Sjolander P, Johansson H, Djupsjobacka M. Spinal and supraspinal effects of activity in ligament afferents. J Electromyogr Kinesiol. Jun 2002;12(3):167-176.

83. Spencer JD, Hayes KC, Alexander IJ. Knee joint effusion and quadriceps reflex inhibition in man. Arch Phys Med Rehabil. 1984;65(4):171-177.

84. Iles JF, Stokes M, Young A. Reflex actions of knee joint afferents during contraction of the human quadriceps. Clin Physiol (Oxford). Sep 1990;10(5):489-500.

85. Palmieri RM, Ingersoll CD, Edwards JE, et al. Arthrogenic muscle inhibition is not present in the limb contralateral to a simulated knee joint effusion. Am J Phys Med Rehabil. Dec 2003;82(12):910-916.

86. Ochi M, Iwasa J, Uchio Y, Adachi N, Sumen Y. The regeneration of sensory neurones in the reconstruction of the anterior cruciate ligament. J Bone Joint Surg Br. Sep 1999;81(5):902-906.

87. Urbach D, Berth A, Awiszus F. Effect of transcranial magnetic stimulation on voluntary activation in patients with quadriceps weakness. Muscle Nerve. Aug 2005;32(2):164-169.

88. Julkunen P, Saisanen L, Danner N, Awiszus F, Kononen M. Within-subject effect of coilto-cortex distance on cortical electric field threshold and motor evoked potentials in transcranial magnetic stimulation. J Neurosci Methods. 2012;206(2):158-164.

89. Todd G, Petersen NT, Taylor JL, Gandevia SC. The effect of a contralateral contraction on maximal voluntary activation and central fatigue in elbow flexor muscles. Exp Brain Res. Jun 2003;150(3):308-313.

90. Gibbons CE, Pietrosimone BG, Hart JM, Saliba SA, Ingersoll CD. Transcranial magnetic stimulation and volitional quadriceps activation. $J$ Athl Train. Nov-Dec 2010;45(6):570579 . 
91. Norte GE, Pietrosimone BG, Hart JM, Hertel J, Ingersoll CD. Relationship between transcranial magnetic stimulation and percutaneous electrical stimulation in determining the quadriceps central activation ratio. Am J Phys Med Rehabil. Dec 2010;89(12):986996.

92. Stern A, Kuenze C, Herman D, Sauer LD, Hart JM. A gender comparison of central and peripheral neuromuscular function after exercise. J Sport Rehabil. Aug 2012;21(3):209217.

93. Oiestad BE, Engebretsen L, Storheim K, Risberg MA. Knee osteoarthritis after anterior cruciate ligament injury: a systematic review. Am J Sports Med. Jul 2009;37(7):14341443.

94. Horak FB, Nashner LM, Diener HC. Postural strategies associated with somatosensory and vestibular loss. Exp Brain Res. 1990;82(1):167-177.

95. Riemann BL GK, Shields EW. Relationship between clinical and forceplate measures of postural stability. J Sport Rehabil. 1999;8(2):71-82.

96. Gribble PA, Hertel J, Plisky P. Using the Star Excursion Balance Test to assess dynamic postural-control deficits and outcomes in lower extremity injury: a literature and systematic review. J Athl Train. 2012;47(3):339-357.

97. Finnoff JT, Peterson VJ, Hollman JH, Smith J. Intrarater and interrater reliability of the Balance Error Scoring System (BESS). PM R. Jan 2009;1(1):50-54.

98. Docherty CL, Valovich McLeod TC, Shultz SJ. Postural control deficits in participants with functional ankle instability as measured by the balance error scoring system. Clin J Sport Med. May 2006;16(3):203-208.

99. Bell DR, Guskiewicz KM, Clark MA, Padua DA. Systematic review of the balance error scoring system. Sports Health. May 2011;3(3):287-295.

100. Donovan L, Hertel J. A new paradigm for rehabilitation of patients with chronic ankle instability. Phys Sportsmed. Nov 2012;40(4):41-51.

101. Valovich McLeod TC, Perrin DH, Guskiewicz KM, Shultz SJ, Diamond R, Gansneder BM. Serial administration of clinical concussion assessments and learning effects in healthy young athletes. Clin J Sport Med. Sep 2004;14(5):287-295.

102. McLeod TC, Armstrong T, Miller M, Sauers JL. Balance improvements in female high school basketball players after a 6-week neuromuscular-training program. J Sport Rehabil. Nov 2009;18(4):465-481.

103. Broglio SP, Zhu W, Sopiarz K, Park Y. Generalizability theory analysis of balance error scoring system reliability in healthy young adults. J Athl Train. Sep-Oct 2009;44(5):497502.

104. Valovich McLeod TC, Barr WB, McCrea M, Guskiewicz KM. Psychometric and measurement properties of concussion assessment tools in youth sports. $J$ Athl Train. Oct-Dec 2006;41(4):399-408.

105. Wilkins JC, Valovich McLeod TC, Perrin DH, Gansneder BM. Performance on the Balance Error Scoring System Decreases After Fatigue. J Athl Train. Jun 2004;39(2):156-161.

106. Susco TM, Valovich McLeod TC, Gansneder BM, Shultz SJ. Balance Recovers Within 20 Minutes After Exertion as Measured by the Balance Error Scoring System. J Athl Train. Sep 2004;39(3):241-246.

107. Hunt TN, Ferrara MS, Bornstein RA, Baumgartner TA. The reliability of the modified Balance Error Scoring System. Clin J Sport Med. Nov 2009;19(6):471-475.

108. Clark RC, Saxion CE, Cameron KL, Gerber JP. Associations between three clinical assessment tools for postural stability. N Am J Sports Phys Ther. Sep 2010;5(3):122-130.

109. Kinzey SJ, Ingersoll CD, Knight KL. The effects of selected ankle appliances on postural control. J Athl Train. Oct 1997;32(4):300-303. 
110. Broglio SP, Monk A, Sopiarz K, Cooper ER. The influence of ankle support on postural control. J Sci Med Sport. May 2009;12(3):388-392.

111. Bressel E, Yonker JC, Kras J, Heath EM. Comparison of static and dynamic balance in female collegiate soccer, basketball, and gymnastics athletes. J Athl Train. Jan-Mar 2007;42(1):42-46.

112. Nieschalk M, Delank KW, Stoll W. [Quantitative evaluation of the Romberg test]. Laryngorhinootologie. Aug 1995;74(8):489-494.

113. Lanska DJ, Goetz CG. Romberg's sign: development, adoption, and adaptation in the 19th century. Neurology. Oct 24 2000;55(8):1201-1206.

114. Lanska DJ. The Romberg sign and early instruments for measuring postural sway. Semin Neurol. Dec 2002;22(4):409-418.

115. Di Fabio RP. Sensitivity and specificity of platform posturography for identifying patients with vestibular dysfunction. Phys Ther. Apr 1995;75(4):290-305.

116. Fanchamps MH, Gensicke H, Kuhle J, Kappos L, Allum JH, Yaldizli O. Screening for balance disorders in mildly affected multiple sclerosis patients. J Neurol. Jul 2012;259(7):1413-1419.

117. Ickenstein GW, Ambach $\mathrm{H}$, Kloditz A, et al. Static posturography in aging and Parkinson's disease. Front Aging Neurosci. 2012;4:20.

118. Maribo T, Stengaard-Pedersen K, Jensen LD, Andersen NT, Schiottz-Christensen B. Postural balance in low back pain patients: Intra-session reliability of center of pressure on a portable force platform and of the one leg stand test. Gait Posture. Jun 2011;34(2):213-217.

119. Roijezon U, Bjorklund M, Djupsjobacka M. The slow and fast components of postural sway in chronic neck pain. Man Ther. Jun 2011;16(3):273-278.

120. Biel A, Dudzinski K. Rehabilitation outcome in patients recovering from reconstruction of the anterior cruciate ligament: a preliminary report. Ortop Traumatol Rehabil. Aug 30 2005;7(4):401-405.

121. Khasnis A, Gokula RM. Romberg's test. J Postgrad Med. Apr-Jun 2003;49(2):169-172.

122. Agrawal Y, Carey JP, Della Santina CC, Schubert MC, Minor LB. Disorders of balance and vestibular function in US adults: data from the National Health and Nutrition Examination Survey, 2001-2004. Arch Intern Med. May 25 2009;169(10):938-944.

123. Agrawal Y, Carey JP, Hoffman HJ, Sklare DA, Schubert MC. The modified Romberg Balance Test: normative data in U.S. adults. Otol Neurotol. Oct 2011;32(8):1309-1311.

124. Treleaven J. Sensorimotor disturbances in neck disorders affecting postural stability, head and eye movement control. Man Ther. Feb 2008;13(1):2-11.

125. Treleaven J. Sensorimotor disturbances in neck disorders affecting postural stability, head and eye movement control--Part 2: case studies. Man Ther. Jun 2008;13(3):266-275.

126. McKeon PO, Hertel J. Systematic review of postural control and lateral ankle instability, part I: can deficits be detected with instrumented testing. J Athl Train. May-Jun 2008;43(3):293-304.

127. Gray G. Lower Extremity Functional Profile. Adrian, MI: Wynn Marketing Inc.; 1995.

128. Gribble P. The Star Excursion Balance Test as a measurement tool. Athlet Ther Today. 2003;8(2):46-47.

129. Plisky PJ, Rauh MJ, Kaminski TW, Underwood FB. Star Excursion Balance Test as a predictor of lower extremity injury in high school basketball players. J Orthop Sports Phys Ther. Dec 2006;36(12):911-919.

130. Gribble PA, Hertel J. Considerations for Normalizing Measures of the Star Excursion Balance Test. Meas Phys Educ Exerc Sci. 2003;7(2):89-100.

131. Hertel J, Miller SJ, Denegar CR. Intratester and intertester reliability during the Star Excursion Balance Tests. J Sport Rehabil. 2000;9(2):104-116. 
132. Robinson RH, Gribble PA. Support for a reduction in the number of trials needed for the star excursion balance test. Arch Phys Med Rehabil. Feb 2008;89(2):364-370.

133. Munro AG, Herrington LC. Between-session reliability of the star excursion balance test. Phys Ther Sport. Nov 2010;11(4):128-132.

134. Hertel J, Braham RA, Hale SA, Olmsted-Kramer LC. Simplifying the star excursion balance test: analyses of subjects with and without chronic ankle instability. J Orthop Sports Phys Ther. Mar 2006;36(3):131-137.

135. Hertel J. Sensorimotor deficits with ankle sprains and chronic ankle instability. Clin Sports Med. Jul 2008;27(3):353-370, vii.

136. Gribble PA, Hertel J, Denegar CR. Chronic ankle instability and fatigue create proximal joint alterations during performance of the Star Excursion Balance Test. Int J Sports Med. Mar 2007;28(3):236-242.

137. McGuine TA, Greene JJ, Best T, Leverson G. Balance as a predictor of ankle injuries in high school basketball players. Clin J Sport Med. Oct 2000;10(4):239-244.

138. Filipa A, Byrnes R, Paterno MV, Myer GD, Hewett TE. Neuromuscular training improves performance on the star excursion balance test in young female athletes. $J$ Orthop Sports Phys Ther. Sep 2010;40(9):551-558.

139. Hoch MC, Staton GS, Medina McKeon JM, Mattacola CG, McKeon PO. Dorsiflexion and dynamic postural control deficits are present in those with chronic ankle instability. $J$ Sci Med Sport. Nov 2012;15(6):574-579.

140. Lysholm M, Ledin T, Odkvist LM, Good L. Postural control--a comparison between patients with chronic anterior cruciate ligament insufficiency and healthy individuals. Scand J Med Sci Sports. Dec 1998;8(6):432-438.

141. McKeon PO, Stein AJ, Ingersoll CD, Hertel J. Altered plantar-receptor stimulation impairs postural control in those with chronic ankle instability. J Sport Rehabil. Feb 2012;21(1):1-6.

142. Negahban H, Mazaheri M, Kingma I, van Dieen JH. A systematic review of postural control during single-leg stance in patients with untreated anterior cruciate ligament injury. Knee Surg Sports Traumatol Arthrosc. May 52013.

143. Pope M, Chinn L, Mullineaux D, McKeon PO, Drewes L, Hertel J. Spatial postural control alterations with chronic ankle instability. Gait Posture. Jun 2011;34(2):154-158.

144. Wikstrom EA, Fournier KA, McKeon PO. Postural control differs between those with and without chronic ankle instability. Gait Posture. May 2010;32(1):82-86.

145. Dauty M, Collon S, Dubois C. Change in posture control after recent knee anterior cruciate ligament reconstruction? Clin Physiol Funct Imaging. May 2010;30(3):187-191.

146. Mohammadi F, Salavati M, Akhbari B, Mazaheri M, Khorrami M, Negahban H. Static and dynamic postural control in competitive athletes after anterior cruciate ligament reconstruction and controls. Knee Surg Sports Traumatol Arthrosc. Aug 2012;20(8):1603-1610.

147. Hale SA, Hertel J, Olmsted-Kramer LC. The effect of a 4-week comprehensive rehabilitation program on postural control and lower extremity function in individuals with chronic ankle instability. J Orthop Sports Phys Ther. Jun 2007;37(6):303-311.

148. Hertel J, Olmsted-Kramer LC. Deficits in time-to-boundary measures of postural control with chronic ankle instability. Gait Posture. Jan 2007;25(1):33-39.

149. McKeon PO, Hertel J. Spatiotemporal postural control deficits are present in those with chronic ankle instability. BMC Musculoskeletal Disord. 2008;9:76.

150. Hertel J, Olmsted-Kramer LC, Challis JH. Time-to-boundary measures of postural control during single leg quiet standing. J Appl Biomech. Feb 2006;22(1):67-73.

151. Schmidt H, Sauer LD, Lee SY, Saliba S, Hertel J. Increased in-shoe lateral plantar pressures with chronic ankle instability. Foot Ankle Int. Nov 2011;32(11):1075-1080. 
152. Drewes LK, McKeon PO, Paolini G, et al. Altered ankle kinematics and shank-rear-foot coupling in those with chronic ankle instability. J Sport Rehabil. Aug 2009;18(3):375388.

153. Morrison KE, Hudson DJ, Davis IS, et al. Plantar pressure during running in subjects with chronic ankle instability. Foot Ankle Int. Nov 2010;31(11):994-1000.

154. Horisberger M, Hintermann B, Valderrabano V. Alterations of plantar pressure distribution in posttraumatic end-stage ankle osteoarthritis. Clin Biomech. Mar 2009;24(3):303-307.

155. Griffin LY, Albohm MJ, Arendt EA, et al. Understanding and preventing noncontact anterior cruciate ligament injuries: a review of the Hunt Valley II meeting, January 2005. Am J Sports Med. Sep 2006;34(9):1512-1532.

156. Barber-Westin SD, Noyes FR. Factors used to determine return to unrestricted sports activities after anterior cruciate ligament reconstruction. Arthroscopy. Dec 2011;27(12):1697-1705.

157. Hewett TE, Ford KR, Myer GD. Anterior cruciate ligament injuries in female athletes: Part 2, a meta-analysis of neuromuscular interventions aimed at injury prevention. Am J Sports Med. Mar 2006;34(3):490-498.

158. Noyes FR, Barber-Westin SD, Fleckenstein C, Walsh C, West J. The drop-jump screening test: difference in lower limb control by gender and effect of neuromuscular training in female athletes. Am J Sports Med. Feb 2005;33(2):197-207.

159. Padua DA, DiStefano LJ, Marshall SW, Beutler AI, de la Motte SJ, DiStefano MJ. Retention of movement pattern changes after a lower extremity injury prevention program is affected by program duration. Am J Sports Med. Feb 2012;40(2):300-306.

160. Padua DA, Marshall SW, Boling MC, Thigpen CA, Garrett WE, Jr., Beutler AI. The Landing Error Scoring System (LESS) Is a valid and reliable clinical assessment tool of jump-landing biomechanics: The JUMP-ACL study. Am J Sports Med. Oct 2009;37(10):1996-2002.

161. Quatman CE, Hewett TE. The anterior cruciate ligament injury controversy: is "valgus collapse" a sex-specific mechanism? Br J Sports Med. May 2009;43(5):328-335.

162. Markolf KL, Burchfield DM, Shapiro MM, Shepard MF, Finerman GA, Slauterbeck JL. Combined knee loading states that generate high anterior cruciate ligament forces. $J$ Orthop Res. Nov 1995;13(6):930-935.

163. DeMorat G, Weinhold P, Blackburn T, Chudik S, Garrett W. Aggressive quadriceps loading can induce noncontact anterior cruciate ligament injury. Am J Sports Med. Mar 2004;32(2):477-483.

164. Beutler A, de la Motte S, Marshall S, Padua D, Boden B. MUSCLE STRENGTH AND QUALITATIVE JUMP-LANDING DIFFERENCES IN MALE AND FEMALE MILITARY CADETS: THE JUMP-ACL STUDY. J Sports Sci Med. 2009;8:663-671.

165. Padua DA, Boling MC, Distefano LJ, Onate JA, Beutler AI, Marshall SW. Reliability of the landing error scoring system-real time, a clinical assessment tool of jump-landing biomechanics. J Sport Rehabil. May 2011;20(2):145-156.

166. Onate J, Cortes N, Welch C, Van Lunen BL. Expert versus novice interrater reliability and criterion validity of the landing error scoring system. J Sport Rehabil. Feb 2010;19(1):41-56.

167. Willson JD, Ireland ML, Davis I. Core strength and lower extremity alignment during single leg squats. Med Sci Sports Exerc. May 2006;38(5):945-952.

168. Munro A, Herrington L, Carolan M. Reliability of 2-dimensional video assessment of frontal-plane dynamic knee valgus during common athletic screening tasks. J Sport Rehabil. Feb 2012;21(1):7-11. 
169. Hewett TE, Myer GD, Ford KR, et al. Biomechanical measures of neuromuscular control and valgus loading of knee predict anterior cruciate ligament injury risk in female athletes: a prospective study. Am J Sports Med. Apr 2005;33(4):492-501.

170. Myer GD, Ford KR, Brent JL, Hewett TE. An integrated approach to change the outcome part I: neuromuscular screening methods to identify high ACL injury risk athletes. $J$ Strength Cond Res. Aug 2012;26(8):2265-2271.

171. Goetschius J, Smith HC, Vacek PM, et al. Application of a clinic-based algorithm as a tool to identify female athletes at risk for anterior cruciate ligament injury: a prospective cohort study with a nested, matched case-control analysis. Am J Sports Med. Sep 2012;40(9):1978-1984.

172. Smith HC, Johnson RJ, Shultz SJ, et al. A prospective evaluation of the Landing Error Scoring System (LESS) as a screening tool for anterior cruciate ligament injury risk. Am J Sports Med. Mar 2012;40(3):521-526.

173. Earl JE, Monteiro SK, Snyder KR. Differences in lower extremity kinematics between a bilateral drop-vertical jump and a single-leg step-down. J Orthop Sports Phys Ther. May 2007;37(5):245-252.

174. Beutler AI, Cooper LW, Kirkendall DT, Garrett WE, Jr. Electromyographic Analysis of Single-Leg, Closed Chain Exercises: Implications for Rehabilitation After Anterior Cruciate Ligament Reconstruction. J Athl Train. Mar 2002;37(1):13-18.

175. Nguyen AD, Shultz SJ, Schmitz RJ, Luecht RM, Perrin DH. A preliminary multifactorial approach describing the relationships among lower extremity alignment, hip muscle activation, and lower extremity joint excursion. J Athl Train. May-Jun 2011;46(3):246256.

176. Powers CM. The influence of altered lower-extremity kinematics on patellofemoral joint dysfunction: a theoretical perspective. J Orthop Sports Phys Ther. Nov 2003;33(11):639646.

177. Piva SR, Fitzgerald K, Irrgang JJ, et al. Reliability of measures of impairments associated with patellofemoral pain syndrome. BMC Musculoskeletal Disord. 2006;7:33.

178. Weir A, Darby J, Inklaar H, Koes B, Bakker E, Tol JL. Core stability: inter- and intraobserver reliability of 6 clinical tests. Clin J Sport Med. Jan 2010;20(1):34-38.

179. Rabin A, Kozol Z. Measures of range of motion and strength among healthy women with differing quality of lower extremity movement during the lateral step-down test. J Orthop Sports Phys Ther. Dec 2010;40(12):792-800.

180. Chmielewski TL, Hodges MJ, Horodyski M, Bishop MD, Conrad BP, Tillman SM. Investigation of clinician agreement in evaluating movement quality during unilateral lower extremity functional tasks: a comparison of 2 rating methods. J Orthop Sports Phys Ther. Mar 2007;37(3):122-129.

181. Chinkulprasert C, Vachalathiti R, Powers CM. Patellofemoral joint forces and stress during forward step-up, lateral step-up, and forward step-down exercises. J Orthop Sports Phys Ther. Apr 2011;41(4):241-248.

182. Noyes FR, Barber SD, Mangine RE. Abnormal lower limb symmetry determined by function hop tests after anterior cruciate ligament rupture. Am J Sports Med. Sep-Oct 1991;19(5):513-518.

183. Fitzgerald GK, Lephart SM, Hwang JH, Wainner RS. Hop tests as predictors of dynamic knee stability. J Orthop Sports Phys Ther. Oct 2001;31(10):588-597.

184. Schmitt LC, Paterno MV, Hewett TE. The impact of quadriceps femoris strength asymmetry on functional performance at return to sport following anterior cruciate ligament reconstruction. J Orthop Sports Phys Ther. Sep 2012;42(9):750-759.

185. Juris PM, Phillips EM, Dalpe C, Edwards C, Gotlin RS, Kane DJ. A dynamic test of lower extremity function following anterior cruciate ligament reconstruction and rehabilitation. J Orthop Sports Phys Ther. Oct 1997;26(4):184-191. 
186. Risberg MA, Holm I, Tjomsland O, Ljunggren E, Ekeland A. Prospective study of changes in impairments and disabilities after anterior cruciate ligament reconstruction. $J$ Orthop Sports Phys Ther. Jul 1999;29(7):400-412.

187. Petschnig $R$, Baron $R$, Albrecht $M$. The relationship between isokinetic quadriceps strength test and hop tests for distance and one-legged vertical jump test following anterior cruciate ligament reconstruction. J Orthop Sports Phys Ther. Jul 1998;28(1):2331 .

188. Logerstedt D, Grindem H, Lynch A, et al. Single-legged hop tests as predictors of selfreported knee function after anterior cruciate ligament reconstruction: the Delaware-Oslo ACL cohort study. Am J Sports Med. Oct 2012;40(10):2348-2356.

189. Logerstedt D, Lynch A, Axe MJ, Snyder-Mackler L. Symmetry restoration and functional recovery before and after anterior cruciate ligament reconstruction. Knee Surg Sports Traumatol Arthrosc. Feb 212012.

190. Munro AG, Herrington LC. Between-session reliability of four hop tests and the agility T-test. J Strength Cond Res. May 2011;25(5):1470-1477.

191. Reinke EK, Spindler KP, Lorring D, et al. Hop tests correlate with IKDC and KOOS at minimum of 2 years after primary ACL reconstruction. Knee Surg Sports Traumatol Arthrosc. Nov 2011;19(11):1806-1816.

192. Bolgla LA, Keskula DR. Reliability of lower extremity functional performance tests. $J$ Orthop Sports Phys Ther. Sep 1997;26(3):138-142.

193. Reid A, Birmingham TB, Stratford PW, Alcock GK, Giffin JR. Hop testing provides a reliable and valid outcome measure during rehabilitation after anterior cruciate ligament reconstruction. Phys Ther. Mar 2007;87(3):337-349.

194. Torry MR, Decker MJ, Ellis HB, Shelburne KB, Sterett WI, Steadman JR. Mechanisms of compensating for anterior cruciate ligament deficiency during gait.Med Sci Sports Exerc. Aug 2004;36(8):1403-1412.

195. Papadonikolakis A, Cooper L, Stergiou N, Georgoulis AD, Soucacos PN. Compensatory mechanisms in anterior cruciate ligament deficiency. Knee Surg Sports Traumatol Arthrosc. Jul 2003;11(4):235-243.

196. DeVita P, Hortobagyi T, Barrier J. Gait biomechanics are not normal after anterior cruciate ligament reconstruction and accelerated rehabilitation. Med Sci Sports Exerc. Oct 1998;30(10):1481-1488.

197. Hart JM, Ko JW, Konold T, Pietrosimone B. Sagittal plane knee joint moments following anterior cruciate ligament injury and reconstruction: a systematic review. Clin Biomech. May 2010;25(4):277-283.

198. Lewek M, Rudolph K, Axe M, Snyder-Mackler L. The effect of insufficient quadriceps strength on gait after anterior cruciate ligament reconstruction. Clin Biomech. Jan 2002;17(1):56-63.

199. Shelburne KB, Torry MR, Pandy MG. Effect of muscle compensation on knee instability during ACL-deficient gait. Med Sci Sports Exerc. Apr 2005;37(4):642-648.

200. McGinley JL, Baker R, Wolfe R, Morris ME. The reliability of three-dimensional kinematic gait measurements: a systematic review. Gait Posture. Apr 2009;29(3):360369.

201. Crompton J, Galea MP, Phillips B. Hand-held dynamometry for muscle strength measurement in children with cerebral palsy. Dev Med Child Neurol. Feb 2007;49(2):106-111.

202. Sisto SA, Dyson-Hudson T. Dynamometry testing in spinal cord injury. $J$ Rehabil Res Dev. 2007;44(1):123-136.

203. Morris SL, Dodd KJ, Morris ME. Reliability of dynamometry to quantify isometric strength following traumatic brain injury. Brain Inj. Dec 2008;22(13-14):1030-1037. 
204. Kelln BM, McKeon PO, Gontkof LM, Hertel J. Hand-held dynamometry: reliability of lower extremity muscle testing in healthy, physically active,young adults. $J$ Sport Rehabil. May 2008;17(2):160-170.

205. Lu TW, Chien HL, Chang LY, Hsu HC. Enhancing the examiner's resisting force improves the validity of manual muscle strength measurements: application to knee extensors and flexors. $J$ Strength Cond Res. Sep 2012;26(9):2364-2371.

206. Lu TW, Hsu HC, Chang LY, Chen HL. Enhancing the examiner's resisting force improves the reliability of manual muscle strength measurements: comparison of a new device with hand-held dynamometry. $J$ Rehabil Med. Nov 2007;39(9):679-684.

207. Stark T, Walker B, Phillips JK, Fejer R, Beck R. Hand-held dynamometry correlation with the gold standard isokinetic dynamometry: a systematic review. PM R. May 2011;3(5):472-479.

208. Oiestad BE, Holm I, Gunderson R, Myklebust G, Risberg MA. Quadriceps muscle weakness after anterior cruciate ligament reconstruction: a risk factor for knee osteoarthritis? Arthritis Care Res (Hoboken). Dec 2010;62(12):1706-1714.

209. Urbach D, Nebelung W, Weiler HT, Awiszus F. Bilateral deficit of voluntary quadriceps muscle activation after unilateral ACL tear. Med Sci Sports Exerc. Dec 1999;31(12):1691-1696.

210. Krishnan C, Williams GN. Quantification method affects estimates of voluntary quadriceps activation. Muscle Nerve. Jun 2010;41(6):868-874.

211. Pietrosimone BG, McLeod MM, Lepley AS. A theoretical framework for understanding neuromuscular response to lower extremity joint injury. Sports Health. Jan 2012;4(1):3135 .

212. Ingersoll CD, Grindstaff TL, Pietrosimone BG, Hart JM. Neuromuscular consequences of anterior cruciate ligament injury. Clin Sports Med. Jul 2008;27(3):383-404, vii.

213. Oiestad BE, Holm I, Aune AK, et al. Knee function and prevalence of knee osteoarthritis after anterior cruciate ligament reconstruction: a prospective study with 10 to 15 years of follow-up. Am J Sports Med. Nov 2010;38(11):2201-2210.

214. Shield A, Zhou S. Assessing voluntary muscle activation with the twitch interpolation technique. Sports Med. 2004;34(4):253-267.

215. Merton PA. Voluntary strength and fatigue. J Physiol. Mar 29 1954;123(3):553-564.

216. Behm DG, St-Pierre DM, Perez D. Muscle inactivation: assessment of interpolated twitch technique. J Appl Physiol. Nov 1996;81(5):2267-2273.

217. Herbert RD, Gandevia SC. Twitch interpolation in human muscles: mechanisms and implications for measurement of voluntary activation. J Neurophysiol. Nov 1999;82(5):2271-2283.

218. Folland JP. Measurement of maximum muscle activation with the interpolated twitch technique. J Appl Physiol. Jul 2009;107(1):365-366; discussion 367-368.

219. Huang YM, Hsu MJ, Lin CH, Wei SH, Chang YJ. The non-linear relationship between muscle voluntary activation level and voluntary force measured by the interpolated twitch technique. Sensors (Basel). 2010;10(1):796-807.

220. Allen GM, Gandevia SC, McKenzie DK. Reliability of measurements of muscle strength and voluntary activation using twitch interpolation. Muscle Nerve. Jun 1995;18(6):593600 .

221. Cooper MA, Herda TJ, Herda AA, Costa PB, Ryan ED, Cramer JT. The reliability of the interpolated twitch technique during submaximal and maximal isometric muscle actions. J Strength Cond Res. Jan 82013.

222. Norregaard J, Lykkegaard JJ, Bulow PM, Danneskiold-Samsoe B. The twitch interpolation technique for the estimation of true quadriceps muscle strength. Clin Physiol. Sep 1997;17(5):523-532. 
223. Behm DG. The interpolated twitch technique may be valid and reliable but limited. $J$ Appl Physiol. Jul 2009;107(1):362; discussion 367-368.

224. Carroll TJ, Cresswell AG. Technical or physiological limitations to the interpolated twitch technique? J Appl Physiol. Jul 2009;107(1):364; discussion 367-368.

225. Racinais S, Girard O. ITT: a tool to demonstrate muscle inactivation rather than to calculate a percentage of activation. J Appl Physiol. Jul 2009;107(1):361; discussion 367368.

226. Van Leeuwen DM, De Ruiter CJ, De Haan A. Effect of stimulation intensity on assessment of voluntary activation. Muscle Nerve. Jun 2012;45(6):841-848.

227. Hart JM, Fritz JM, Kerrigan DC, Saliba EN, Gansneder BM, Ingersoll CD. Quadriceps inhibition after repetitive lumbar extension exercise in persons with a history of low back pain. J Athl Train. Jul-Sep 2006;41(3):264-269.

228. Hurley MV, Jones DW, Newham DJ. Arthrogenic quadriceps inhibition and rehabilitation of patients with extensive traumatic knee injuries. Clin Sci (Lond). Mar 1994;86(3):305-310.

229. Norte GE, Pietrosimone BG, Hart JM, Hertel J, Ingersoll CD. Relationship between transcranial magnetic stimulation and percutaneous electrical stimulation in determining the quadriceps central activation ratio. Am J Phys Med Rehabil. Dec 2010;89(12):986996.

230. Park J, Hopkins JT. Within- and between-session reliability of the maximal voluntary knee extension torque and activation. Int J Neurosci. Jan 2013;123(1):55-59.

231. Rice DA, McNair PJ. Quadriceps arthrogenic muscle inhibition: neural mechanisms and treatment perspectives. Semin Arthritis Rheum. Dec 2010;40(3):250-266.

232. Hopkins J, Ingersoll CD, Edwards J, Klootwyk TE. Cryotherapy and Transcutaneous Electric Neuromuscular Stimulation Decrease Arthrogenic Muscle Inhibition of the Vastus Medialis After Knee Joint Effusion. J Athl Train. Mar 2002;37(1):25-31.

233. Kuenze C, Hertel J, Hart JM. Effects of Exercise on Lower Extremity Muscle Function Following ACL Reconstruction. J Sport Rehabil. Jul 42012.

234. Kim KM, Hart JM, Hertel J. Influence of body position on fibularis longus and soleus Hoffmann reflexes. Gait Posture. Jan 2013;37(1):138-140.

235. Palmieri RM, Ingersoll CD, Hoffman MA, et al. Arthrogenic muscle response to a simulated ankle joint effusion. Br J Sports Med. Feb 2004;38(1):26-30.

236. Hopkins JT, Stencil R. Ankle cryotherapy facilitates soleus function. J Orthop Sports Phys Ther. Dec 2002;32(12):622-627.

237. Park J, Hopkins JT. Induced anterior knee pain immediately reduces involuntary and voluntary quadriceps activation. Clin J Sport Med. Jan 2013;23(1):19-24.

238. Capaday C. Neurophysiological methods for studies of the motor system in freely moving human subjects. J Neurosci Methods. Jun 27 1997;74(2):201-218.

239. Palmieri RM, Weltman A, Edwards JE, et al. Pre-synaptic modulation of quadriceps arthrogenic muscle inhibition. Knee Surg Sports Traumatol Arthrosc. Jul 2005;13(5):370376.

240. Hopkins JT, Wagie NC. Intrasession and intersession reliability of the quadriceps Hoffmann reflex. Electromyogr Clin Neurophysiol. Mar 2003;43(2):85-89.

241. Palmieri RM, Hoffman MA, Ingersoll CD. Intersession reliability for H-reflex measurements arising from the soleus, peroneal, and tibialis anterior musculature. Int $J$ Neurosci. Jul 2002;112(7):841-850.

242. Upton AR, McComas AJ, Sica RE. Potentiation of "late" responses evoked in muscles during effort. J Neurol Neurosurg Psychiatry. Dec 1971;34(6):699-711.

243. Hultborn $\mathrm{H}$, Pierrot-Deseilligny E. Changes in recurrent inhibition during voluntary soleus contractions in man studied by an H-reflex technique. $J$ Physiol. Dec 1979;297(0):229-251. 
244. Pensini M, Martin A. Effect of voluntary contraction intensity on the H-reflex and Vwave responses. Neurosci Lett. Sep 9 2004;367(3):369-374.

245. Vila-Cha C, Falla D, Correia MV, Farina D. Changes in H reflex and V wave following short-term endurance and strength training. J Appl Physiol. Jan 2012;112(1):54-63.

246. Ekblom MM. Improvements in dynamic plantar flexor strength after resistance training are associated with increased voluntary activation and V-to-M ratio. $J$ Appl Physiol. Jul 2010;109(1):19-26.

247. Fimland MS, Helgerud J, Gruber M, Leivseth G, Hoff J. Functional maximal strength training induces neural transfer to single-joint tasks. Eur J Appl Physiol. Sep 2009; 107(1):21-29.

248. Godin G, Shephard RJ. A simple method to assess exercise behavior in the community. Can J Appl Sport Sci. Sep 1985;10(3):141-146.

249. Solstad GM, Fimland MS, Helgerud J, Iversen VM, Hoff J. Test-retest reliability of vwave responses in the soleus and gastrocnemius medialis. J Clin Neurophysiol. Apr 2011;28(2):217-221.

250. Duclay J, Martin A. Evoked H-reflex and V-wave responses during maximal isometric, concentric, and eccentric muscle contraction. J Neurophysiol. Nov 2005;94(5):35553562 .

251. Aagaard P, Simonsen EB, Andersen JL, Magnusson P, Dyhre-Poulsen P. Neural adaptation to resistance training: changes in evoked $\mathrm{V}$-wave and H-reflex responses. $J$ Appl Physiol. Jun 2002;92(6):2309-2318.

252. Gondin J, Duclay J, Martin A. Soleus- and gastrocnemii-evoked V-wave responses increase after neuromuscular electrical stimulation training. J Neurophysiol. Jun 2006;95(6):3328-3335.

253. Weiss LD SJ, Weiss J. Easy EMG: Butterworth-Heinemann. 2004.

254. Mitsiokapa EA, Mavrogenis AF, Antonopoulos D, Tzanos G, Papagelopoulos PJ. Common peroneal nerve palsy after grade I inversion ankle sprain. J Surg Orthop Adv. Winter 2012;21(4):261-265.

255. Hayes DW, Jr., Mandracchia VJ, Webb GE. Nerve injury associated with plantarflexioninversion ankle sprains. Clin Podiatr Med Surg. Apr 2000;17(2):361-369, vi-vii.

256. Kleinrensink GJ, Stoeckart R, Meulstee J, et al. Lowered motor conduction velocity of the peroneal nerve after inversion trauma. Med Sci Sports Exerc. Jul 1994;26(7):877-883.

257. Todd G, Taylor JL, Gandevia SC. Measurement of voluntary activation of fresh and fatigued human muscles using transcranial magnetic stimulation. $J$ Physiol. Sep 1 2003;551(Pt 2):661-671.

258. Fisher BE, Lee YY, Pitsch EA, et al. Method for assessing brain changes associated with gluteus maximus activation. J Orthop Sports Phys Ther. Apr 2013;43(4):214-221.

259. Terao Y, Ugawa Y. Basic mechanisms of TMS. J Clin Neurophysiol. Aug 2002;19(4):322-343.

260. Hallett M. Transcranial magnetic stimulation and the human brain. Nature. Jul 13 2000;406(6792):147-150.

261. Tallus J, Lioumis P, Hamalainen H, Kahkonen S, Tenovuo O. Long-lasting TMS motor threshold elevation in mild traumatic brain injury. Acta Neurol Scand. Sep 2012;126(3):178-182.

262. Pietrosimone BG, Gribble PA. Chronic ankle instability and corticomotor excitability of the fibularis longus muscle. J Athl Train. 2012;47(6):621-626.

263. Livingston $\mathrm{SC}$, Ingersoll $\mathrm{CD}$. Intra-rater reliability of a transcranial magnetic stimulation technique to obtain motor evoked potentials. Int J Neurosci. Feb 2008;118(2):239-256.

264. Tallent J, Goodall S, Hortobagyi T, St Clair Gibson A, French DN, Howatson G. Repeatability of corticospinal and spinal measures during lengthening and shortening contractions in the human tibialis anterior muscle. PloS one. 2012;7(4):e35930. 